\title{
A review of the genus Scathophaga Meigen, 1803 (Diptera: Scathophagidae) of Russia
}

\author{
Обзор видов рода Scathophaga Meigen, 1803 (Diptera: \\ Scathophagidae) фауны России
}

\author{
A.L. Ozerov ${ }^{1,3}$, M.G. Krivosheina ${ }^{2}$

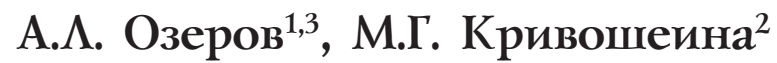

\begin{abstract}
${ }^{1}$ Zoological Museum, Moscow Lomonosov State University, Bol'shaya Nikitskaya Str. 2, Moscow 125009, Russia. E-mail: ozerov2455@rambler.ru

1Зоологический музей, Московский государственный университет им. М.В. Ломоносова, Большая Никитская ул., 2, Москва 125009, Россия.

${ }^{2}$ A.N. Severtsov Institute of Ecology and Evolution, Russian Academy of Sciences, Leninsky prospect, 33, Moscow 119071, Russia. Email: dipteramarina@rambler.ru

${ }^{2}$ Институт проблем экологии и эволюции им. А.Н. Северцова РАН, Ленинский проспект, 33, Москва 119071, Россия.

${ }^{3}$ corresponding author
\end{abstract}

KEYWORDS: Diptera, Scathophagidae, dung flies, Scathophaga, Russia, review, new record.

КЛЮЧЕВЫЕ СЛОВА: Diptera, Scathophagidae, Scathophaga, Russia, новые данные по распространению.

ABSTRACT. Flies of the genus Scathophaga Meigen, 1803 of Russia, which includes 29 species, are reviewed. Generic and species descriptions and key for determination of species are given, and data on distributions are summarized. Scathophaga incompleta Ozerov et Krivosheina, 2020 is registered in Turkmenistan for the first time.

РЕЗЮМЕ. Дан обзор двукрылых рода Scathophaga Meigen, 1803 фауны России, включающего 29 видов. Приведены диагноз рода, описания видов и ключ для определения видов рода Scathophaga России, а также суммированы данные по распространению видов рода на территории России. Вид Scathophaga incompleta Ozerov et Krivosheina, 2020 впервые отмечен на территории Туркмении.

\section{Introduction}

Scathophaga Meigen, 1803 is the second largest genus after Cordilura Fallén, 1810 within the family Scathophagidae and consists of 66 species distributed in the Northern Hemisphere: 45 species are known in Palaearctic [Šifner, 2008, 2013, 2018, 2020; Ozerov, 2013, 2017; Ozerov, Krivosheina, 2019, 2020]) and 10 in Nearctic [Vockeroth, 1965, 1987; Šifner, 2017 ], with 11 species with a Holarctic distribution; 4 species are recorded in Oriental Region [Sun, 1996], 3 species in Neotropical Region [Albuquerque, 1984] and 5 species in Afrotropical Region [Ozerov, 2010a, 2012].

23 species were described from the territory of Russia [Holmgren, 1880, 1883; Becker, 1894, 1897, 1900, 1907, 1915; Coquillett, 1901; Szilády, 1926; Ozerov, 1996, 2010b, 2013, 2017; Ozerov, Krivosheina, 2019, 2020], from which 12 names are valid and 11 names are synonyms.

Faunistic reviews of some districts of Russia are published for the European part [Gorodkov, 1972], Arctic zone [Engelmark, 1999; Ozerov, Barkalov, 2014], Russian Far East [Ozerov, Krivosheina, 2014]; Arkhangelsk Oblast [Ovchinnikov, Makarova, 2016], Karelia [Polevoi, 1997; Humala, Polevoi, 2009], Mordovia [MacGowan et al., 2021], Volga Region [Ovchinnikov, 2004], Yakutia [Sychevskaya, 1972; Veselkin, 1985; Verves et al., 1990; Bagachanova et al., 2016] and Kamchatka [Hendel, 1930], but no general work has been fulfilled.

Gorodkov published the distribution areas of species on the territory of the former USSR and separately on its European part for three most common in Russia species of Scathophaga: S. furcata (Say, 1823) [Gorodkov, 1978], S. spurca (Meigen, 1826) (as S. suilla (Fabricius, 1794)) [Gorodkov, 1980] and S. stercoraria (Linnaeus, 1758) [Gorodkov, 1981].

At the present time the fauna of Russia includes 29 species.

The limits of this genus are not clear. As investigations of Bernasconi et al. [2000, 2001] showed,

How to cite this article: Ozerov A.L., Krivosheina M.G. 2021. A review of the genus Scathophaga Meigen, 1803 (Diptera: Scathophagidae) of Russia // Russian Entomol. J. Vol.30. No.2. P.201-246. doi: 10.15298/ rusentj.30.2.16 

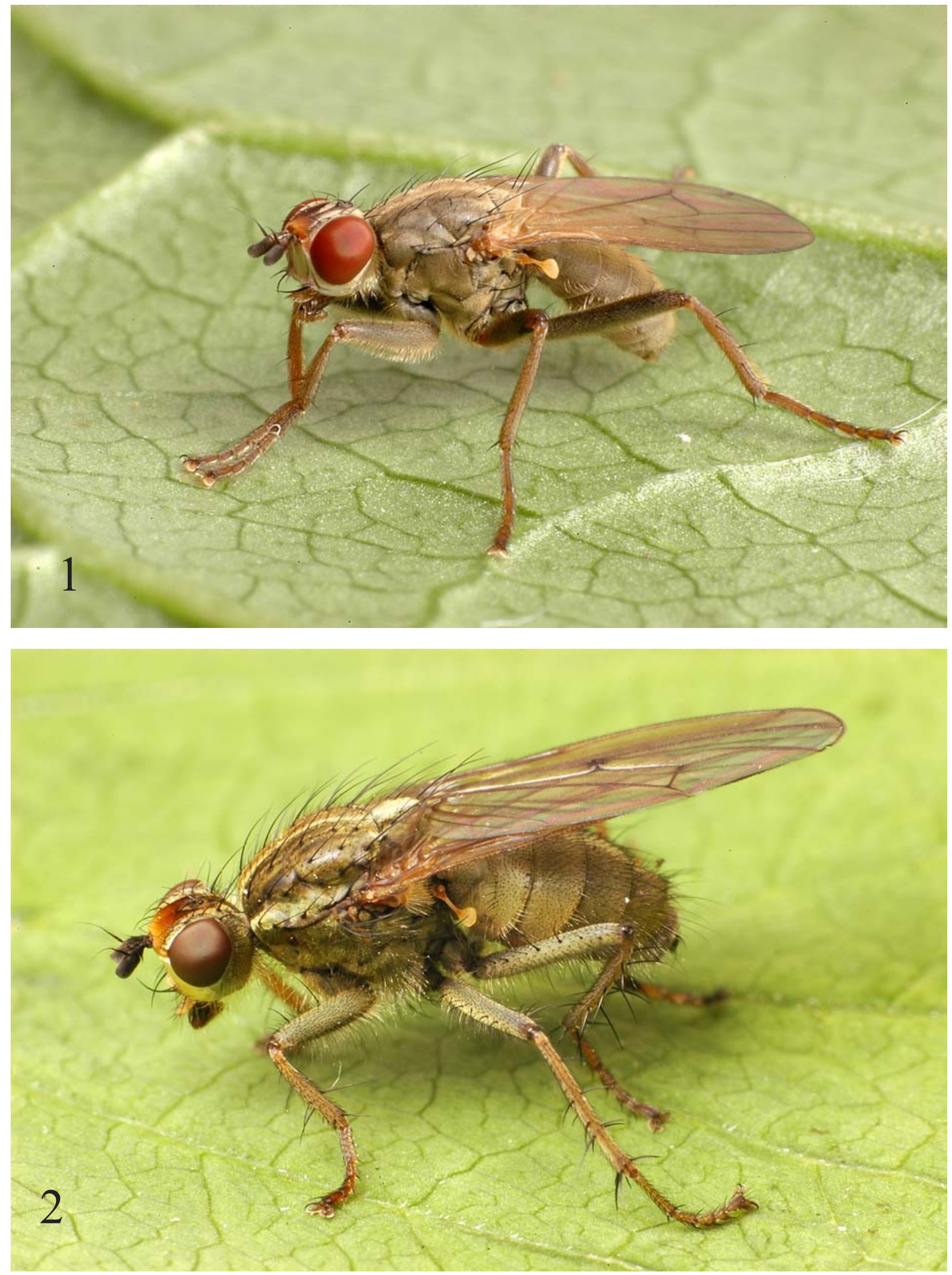

Figs 1-2. Males of Scathophaga obscura (Fallén) (1) and Scathophaga stercoraria (Linnaeus) (2). Photographs by D.I. Gavryushin. 2 after Ozerov \& Freidberg, 2011, fig. 24.

Рис. 1-2. Самцы Scathophaga obscura (Fallén) (1) и Scathophaga stercoraria (Linnaeus) (2). Фото Д.И. Гаврюшина. 2 — по Ozerov \& Freidberg, 2011, fig. 24. 
Scathophaga sensu Gorodkov [1986] and Vockeroth [1987] is not monophyletic. According to the classifications of Šifner [2008] and de Jong [2000] the species in Russia should be assigned to two genera: Scathophaga (most species) and Conisternum Strobl, 1894 (buryatica Ozerov \& Krivosheina, 2019, decipiens (Haliday in Curtis, 1832), incompleta Ozerov et Krivosheina, 2020, lapponica (Ringdahl, 1920), mollis (Becker, 1894), nigripalpis (Becker, 1907), obscura (Fallén, 1819), and tinctinervis (Becker, 1894)). However, a consideration of all the species of Scathophaga sensu lato and Conisternum reveals that the taxonomic borders between Scathophaga and Conisternum are not clear. Therefore in the present work we follow Gorodkov's and Vockeroth's point of view and refer all Russian species to a single genus, i.e., Scathophaga sensu lato.

Larvae of Scathophaga species are carnivorous in dung and in rotten seaweed on the coast [Ferrar, 1987].

\section{Material and methods}

The specimens examined for this study are deposited in the Zoological Museum, Moscow State University, Russia (ZMUM) and Zoological Institute of Russian Academy of Sciences, St. Petersburg, Russia (ZISP). We also studied some material from the Institute of Systematics and Ecology of Animals, Russian Academy of Sciences, Siberian Branch, Novosibirsk, Russia (ISEA) and from Institute of Biology and Soil Sciences, Russian Academy of Sciences, Far Eastern Branch, Vladivostok, Russia (IBSS).

Some of the previously used material kept in ZMUM from Siberia [Ozerov, Barkalov, 2014] and Far East of Russia [Ozerov, 2010b; Ozerov, Krivosheina, 2014] was reexamined by us, but we do not duplicate the list of the specimens and give reference to these works.

The majority of original Russian geographical names are given in transliteration, but names of some large geographical regions (e.g., Altai (=Altai Krai + Altai Republic), Krasnoyarsk Krai, Primorsky Krai, Sakha Republic (=Yakutia), Zabaykalsky Krai) follow these indicated in Google Earth software. More detailed data on the distributions of species in Europe may be found in the work by Šifner [2008], and in North America in the work by Vockeroth [1965].

The terminology used in the generic and species descriptions follows Séguy [1952 (scapular seta)], McAlpine [1981], Cumming \& Wood [2009], and Stuckenberg [1999 (postpedicel)].

\section{Taxonomic results}

\section{Scathophaga Meigen, 1803}

Scathophaga Meigen, 1803: 277. Gender: feminine. Typespecies: Musca merdaria Fabricius, 1794, by monotypy [=Musca stercoraria (Linnaeus, 1758)].

Scopeuma Meigen, 1800: 36. Gender: feminine. Type-species: Musca merdaria Fabricius, 1794, by designation of Coquillett, 1901) [= Musca stercoraria Linnaeus]. Surpressed by I.C.Z.N., 1963, Opinion 678.
Pyropa Illiger, 1807: 475. Gender: feminine. Type-species: Musca stercoraria Linnaeus, by designation of Vockeroth, 1965. Amina Robineau-Desvoidy, 1830: 629. Gender: feminine. Typespecies: Amina parisiensis Robineau-Desvoidy, 1830, by monotypy. Scatina Robineau-Desvoidy, 1830: 629. Gender: feminine. Type-species: Scatina claripennis Robineau-Desvoidy, 1830, by monotypy.

Conisternum Strob1, 1894: 79. Gender: neuter. Type-species: Cordylura obscura Fallén, 1819, by monotypy.

Coniosternum Becker, 1894: 176. Gender: neuter. Type-species: Cordylura obscura Fallén, 1819, by original designation.

Scatophagella Szilády, 1926: 596. Gender: feminine. Typespecies: Scatophagella pubescens Szilády, 1926, by original designation; doubtful species. 5].

Koniosternum, error for Coniosternum Becker [Becker, 1894:

Scatophaga: unjustified emend.

Adult Scathophaga can be distinguished from the other genera of Scathophagidae by the following combination of characters: 1) anepisternum covered with hairs completely or almost completely, with hairs posterior to anterior spiracle (Fig. 6), 2) postmetacoxal bridge absent, 3) katepisternum with one long seta in posterodorsal corner.

Scathophaga species are slender, small to mediumsized flies (about 4.2-10.0 mm long) (Figs 1-3).

Head subspherical; eyes moderately large, dichoptic in both sexes (Figs 1-5). Frontal vitta and fronto-orbital plate distinct. 3-5 orbitals, 2-7 frontals, 1 ocellar, 1 inner vertical, 1 outer vertical, 1 postocellar (divergent) setae, and 1-3 pairs of strong vibrissae and 3-5 pairs of subvibrissae present. Postcranium greyish dusted, usually covered with black setae and setulae in upper third or half, and with pale hairs in lower part. Postpedicel with roundish apical angle, approximately twice as long as wide. Antenna with arista bare to plumose (Figs 1-4). Palpus slender, slightly broadened towards apex (Figs 4, 5).

Thorax greyish dusted. Scutum with following setae: acrostichals usually setulose in two rows or not differentiated from the other hairs on scutum and they don't form rows; 0-1 scapular (usually small); number of dorsocentrals varies, but $2+3$ in most species; intraalars $(0-1)+(0-2)$; supra-alars $(0-1)+(1-3)$, but $1+2$ in most species; postpronotals $1-2$; notopleurals 2 ; postalars 2. Proepisternum covered with fine hairs, with $0-1$ setae near ventral margin. Proepimeron with $0-1$ seta ventral to spiracle. Anepisternum covered with hairs completely or almost completely, with hairs posterior to anterior spiracle and with 2-3 well-developed setae near posterior margin. Katepisternum covered with hairs completely, with one strong seta in posterodorsal corner, and usually with long hairs posteriorly. Anepimeron bare or covered with several setae. Postmetacoxal bridge absent. Scutellum of most species with a pair of strong discal and a pair of strong apical setae (Figs 2, 3).

Legs long and slender. Male legs of some species (e.g., S. callida (Haliday, 1832), S. dasythrix (Becker, 1894)) with dense, long hairs, and legs of females much less hairy.

Wing well-developed (only S. exalata Ozerov, 1996 with reduced wings), clear or tinged with brownish.Veins blackish; vein $\mathrm{R}_{1}$ without setulae on apical third of dorsal surface; crossveins r-m and d-m often darkened 

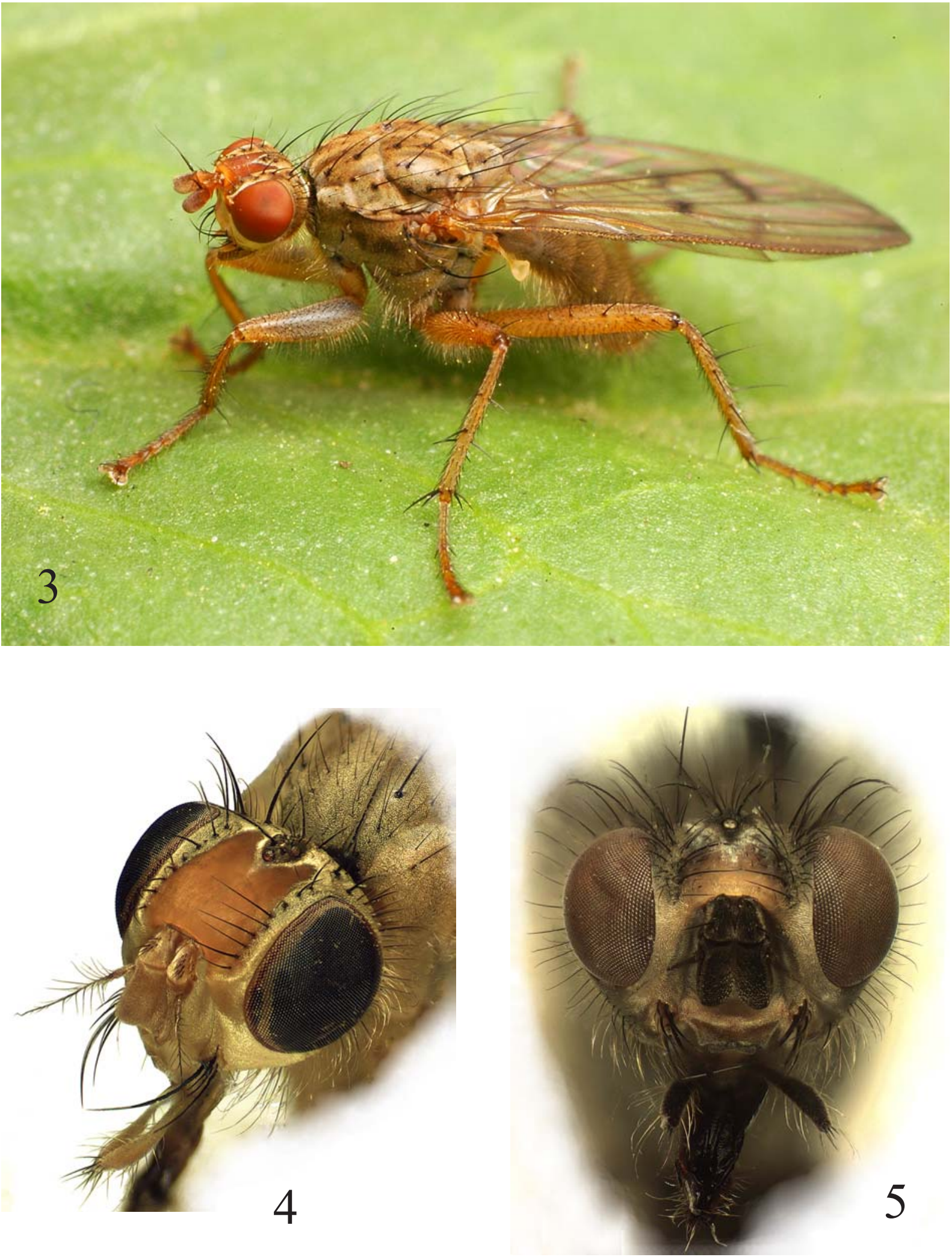

Figs 3-5. Scathophaga furcata (Say) (3), S. taeniopa (Rondani) (4) and S. nigripalpis (Becker) (5): 3 - female adult (Photograph by D.I. Gavryushin); 4, 5 - head.

Pис. 3-5. Scathophaga furcata (Say) (3), S. taeniopa (Rondani) (4) and S. nigripalpis (Becker) (5): 1 — самка (Фото Д.И. Гаврюшина); 4, 5 - голова. 

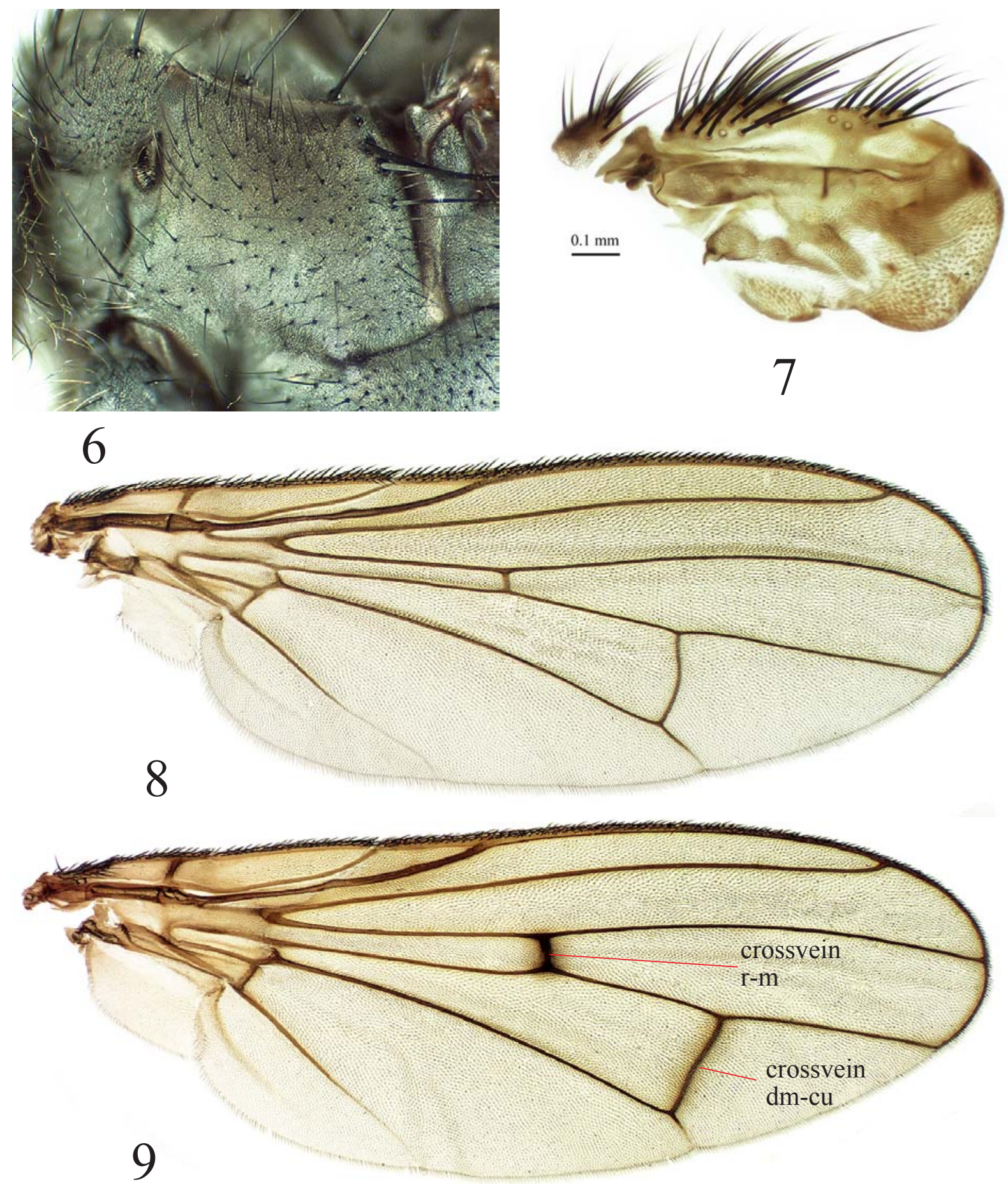

Figs 6-9. Scathophaga litorea (Fallén) (6, 8), S. exalata Ozerov (7) and S. incola (Becker) (9): 6 - anepisternum; 7-9 — wing. Pис. 6-9. Scathophaga litorea (Fallén) (6, 8), S. exalata Ozerov (7) и S. incola (Becker) (9): 6 - анэпистерн; 7-9 — крыло.

(Fig. 9). Calypters whitish, margins of calypters and halteres from whitish to brownish or blackish, but usually yellowish.

Abdomen long, subcylindrical, usually greyish dusted. Female tergites $2-6$ often with a row of marginal setae or setulae. Male sternite 4 varies in form (Figs 14-42). Form of male sternite 5 also varies greatly (e.g., Figs 43, 52, 61, 67, 79, 91). Surstyli symmetrical, simple (e.g., Figs 51, 78) or bifurcate (e.g., Figs 45, $57)$; cerci fused usually medially forming cercal plate (e.g., Figs 69, 75). Ovipositor short and compact (Figs $10-13$. Tergite 6 forming narrow semiring, partly situated under large tergite 5. Sternite 6 in form of pentagonal or ovoid plate. Tergite 7 sclerotized completely 
or sclerotized laterally only and membranous dorsally. Sternite 7 simple and usually has the form of ovoid plate (most species) or divided into three sclerites (e.g., S. apicalis (Curtis in Ross, 1835), S. dasytrix, S. furcata, S. litorea (Fallén, 1819) (Fig. 13), S. stercoraria (Linnaeus, 1758)). Tergite 8 in form of heavily sclerotized sclerite completely, or sclerotized sclerite with deep membranous dorsal emargination in distal part, or two separated lateral sclerites. Sternite 8 simple, as flat dorsoventrally triangular plate, or with deep median notch, compressed in distal part and flattened dorsoventrally in basal part, or divided medially into two sclerites and looking like triangular, spatulate or lanceolate plate laterally, or two simple small oval sclerites. Proctiger shifted dorsally and represented by small entire epiproct, a pair of small cerci, and hypoproct.

\section{Scathophaga apicalis (Curtis in Ross, 1835)} Figs 14, 43-45, 140.

apicalis Curtis in Ross, 1835: LXXX (Scatophaga). Typelocality: not given (Boothia Pen. to Greendland).

arctica Becker, 1897: 398 (Scatophaga). Type-locality: Malye Karmakuly (Russia: Novaya Zemlya).

lanata Lundbeck, 1901: 294 (Scatophaga). Type-locality: "Grønland... Østkysten ved Hekla Havn og. Gaaselandet" (Grenland: Scorsby Sund nr Hekla Havn and Gaaseland).

perfecta Becker, 1907: 3 (Scatophaga). Type-locality: "LenaMündung, Charanlach-gebirge, Cap Elijdep" [arctic coast of Yakutia] (Russia).

vulpinum Ringdahl, 1936: 173 (Scopeuma). Junior primary homonym of Scopeuma vulpina Coquillett, 1898. Type-locality: "Lappland bei Torneträsk in der Regio Alpina" (Sweden).

villosiventre Ringdahl, 1937: 38 (new name for Scopeuma vulpinum Ringdahl, 1936).

REMARK. This species was recorded in Russia on arctic coast from European part (Yugorsky Peninsula, Novaya Zemlya) [Becker, 1897: 398, as $S$. arctica; Gorodkov, 1986: 29], Siberia (Taimyr Peninsula) [Ozerov, Barkalov, 2014: 563], Yakutia [Becker, 1907: 3, as S. perfecta; Verves et al., 1990: 141; Bagachanova et al., 2016: 781], Chukotka [Gorodkov, 1986: 29; Ozerov, Krivosheina, 2014: 218, as $S$. multisetosa].

MATERIAL EXAMINED. Chukotka: $5 \mathrm{~km} \mathrm{~N}$ of Egvekinot

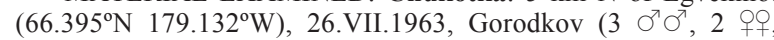
ZISP); Iul'tin $\left(67.866^{\circ} \mathrm{N} 178.733^{\circ} \mathrm{W}\right), 20 . \mathrm{VII} .1963$, Gorodkov (2 $\sigma^{7} \sigma^{7}$, ZISP); Vrangel I. (71.223 $\left.\mathrm{N} 179.439^{\circ} \mathrm{W}\right)$, surrounding area of Mt. Aternon, 24.VII.1939, Portenko (8 $\sigma^{7} \sigma^{7}, 4$ 우, ZISP); Vrangel I. (71.223 $\left.\mathrm{N} 179.439^{\circ} \mathrm{W}\right)$, Tundrovaya env., 18.VII.1972, Gorodkov $\left(8 \sigma^{7} \sigma^{7}, 12\right.$ 우, ZISP); Vrangel I., Somnitel'naya Bay $\left(70.894^{\circ} \mathrm{N}\right.$

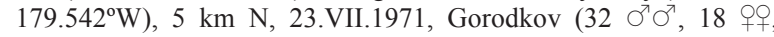
ZISP); Vrangel I., Somnitel'naya Bay $\left(70.95^{\circ} \mathrm{N} 179.633^{\circ} \mathrm{W}\right), 8-$ 17.VI.2015, O.A. Khruleva (1 $\sigma^{7}$, ZMUM); Vrangel I., average flow of the River Mamontovaya $\left(71.166^{\circ} \mathrm{N} 179.75^{\circ} \mathrm{W}\right), 1-2$, 23.VII.2015, O.A. Khruleva (13 ठ $\sigma^{\top}, 13$ 우, ZMUM); Vrangel I., Mt Mineev $\left(71.00^{\circ} \mathrm{N} 179.516^{\circ} \mathrm{W}\right), 18-19$.VII.2015, O.A. Khruleva (2 $\sigma^{7} \sigma^{7}$, ZMUM); Vrangel I., Rodzhers Bay $\left(70.979^{\circ} \mathrm{N} 178.416^{\circ} \mathrm{W}\right)$, 18.VII.1971, Gorodkov (5 $\sigma^{7} \sigma^{7}, 1$ \% , ZISP); Vrangel I., spurs of Mt Pervaya $\left(71.15^{\circ} \mathrm{N} 179.45^{\circ} \mathrm{W}\right), 13$.VII.2015, O.A. Khruleva (1 $\sigma^{7}$, ZMUM); Vrangel I., upper course of the River Neizvestnaya $\left(71.216^{\circ} \mathrm{N} 179.316^{\circ} \mathrm{W}\right), 22-24 . V I ., 8-10 . V I I .2015$, O.A. Khruleva (9 $\sigma^{\top} \sigma^{\top}, 5$ +क, ZMUM); Krasnoyarsk Krai: $13.5 \mathrm{~km}$ SSE of Dikson $\left(73.39^{\circ} \mathrm{N} 80.66^{\circ} \mathrm{E}\right), 18$. VII.2012, V.K. Zinchenko (1 $\sigma^{7}$, ZISP); Dikson $\left(73.508^{\circ} \mathrm{N} 80.529^{\circ} \mathrm{E}\right)$, 8.VIII.1967, Gorodkov (7 $\sigma^{7} \bigcirc^{7}, 14$ 우, ZISP); Taymyr, the lower reaches of the River Lenivaya $\left(74.966^{\circ} \mathrm{N} 89.916^{\circ} \mathrm{E}\right)$, on flowers of Sieversia glacialis, 4.VII.1980, Yu.I. Chernov (2 $\sigma^{7} \Im^{7}$, ZMUM); Yakutia: Tiksi env., Lake Sevast'yan $\left(71.543^{\circ} \mathrm{N} 128.852^{\circ} \mathrm{E}\right), 9$.VII. and 10.VIII.1957, Gorodkov ( $8 \sigma^{7} \sigma^{7}, 5$ 우, ZISP). See also Ozerov \& Krivosheina [2014: 218, as S. multisetosa] and Ozerov \& Barkalov [2014: 563].

DESCRIPTION. Male. Female. Body-length 4.3$8.6 \mathrm{~mm}$

Male thorax, abdomen and legs covered with dense, furry, blackish or brownish hairs. Female much less hairy, especially abdomen and legs.

Head. Frontal vitta reddish-orange, with delicate whitish reflection; fronto-orbital plate black, greyish dusted. Face, parafacial and gena reddish-orange, with delicate whitish reflection. Postcranium black. 3 orbital and 4-5 frontal setae present. Scapus and pedicel reddish-orange. Postpedicel black, approximately 2 times as long as wide. Arista bare. Palpus yellow.

Thorax black, densely greyish dusted. Acrostichals not differentiated from the other long hairs on scutum, dorsocentrals $2+3$, intra-alars $1+2$, supra-alars $1+2$, postpronotals 2. Anepimeron bare. Scutellum black, greyish dusted, covered with dense hairs, with a pair of strong basal scutellar and a pair of strong apical scutellar setae.

Legs greyish dusted. Femora and coxae from yellow completely to blackish. Tibiae and tarsi yellowish. All femora covered with hairs, but without conspicuous setae, only mid femur with 1 preapical posterior seta. Fore tibia with 2-3 dorsal, 1-2 posterior, 1 preapical anterodorsal, and 1 posterior apical setae. Mid tibia with 2-3 posterodorsal, 1-2 anterodorsal, 1-2 posteroventral setae and a ring of apicals in both sexes, additionally with 1 ventral seta in female. Hind tibia with 2-3 posterodorsal, 3 anterodorsal, 1 preapical dorsal, 1 preapical anterodorsal and 1 apical anteroventral setae (Fig. 140).

Wing tinged with brownish; crossveins r-m and dmcu usually darkened.

Abdomen black, greyish dusted. Male sternite 4 approximately 1.5 times as long as wide (Fig. 14), Male sternite 5, epandrium, cercal plate and surstyli as in Figs 43-45.

DISTRIBUTION. Russia: north (arctic coast) from Kola Peninsula to Chukotka. - Europe (Sweden); North America.

Scathophaga buryatica Ozerov et Krivosheina, 2019 Figs 15, 46-48, 130.

buryatica Ozerov, Krivosheina, 2019: 102 (Scathophaga). Type-locality: Samarta $\left(52.094^{\circ} \mathrm{N} 101.137^{\circ} \mathrm{E}\right), 40 \mathrm{~km}$ NO of Mondy (Russia: Buryatia).

REMARK. This species known from two males from Buryatia only [Ozerov, Krivosheina, 2019: 102].

DESCRIPTION. Male. Length of body 5.2-6.2 mm.

Head. Frontal vitta yellow, with delicate whitish reflection; face and gena yellow, with whitish reflection; fronto-orbital plate greyish dusted. Postcranium blackish. 3 orbital and 4-5 frontal setae present. Antenna black; postpedicel about twice as long as wide. Arista bare. Palpus yellow. 

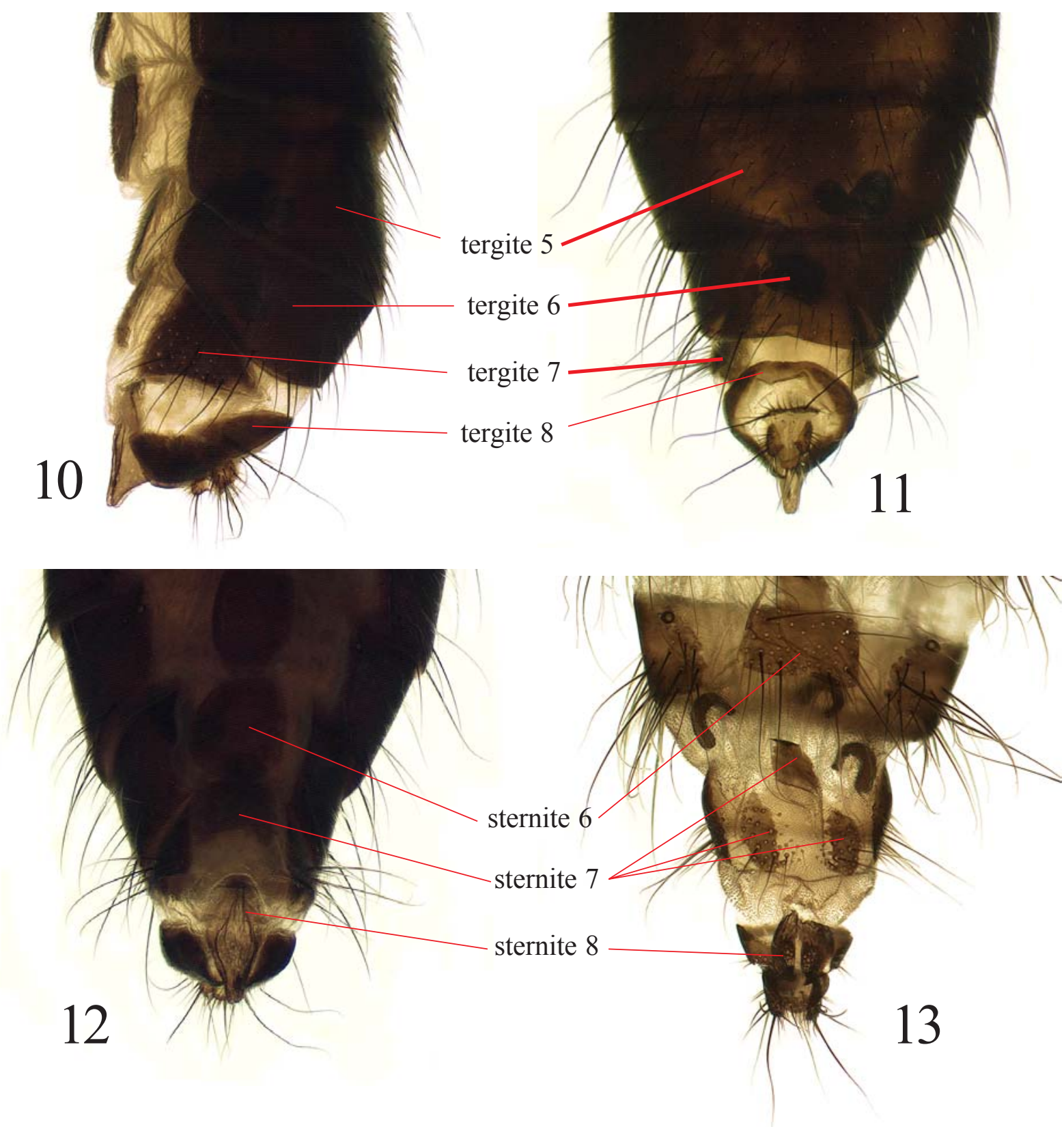

Figs 10-13. Scathophaga lapponica (Ringdahl) (10-12) and Scathophaga litorea (Fallén) (13): 10 — end of female abdomen, lateral view; 11 - same, dorsal view; 12,13 - same, ventral view.

Рис 10-13. Scathophaga lapponica (Ringdahl) (10-12) и Scathophaga litorea (Fallén) (13): 10 — конец брюшка самки, сбоку; 11 — то же, сверху; 12, 13 - то же, снизу.

Thorax. Completely black, dense pale grey dusted. Acrostichals setulose in two rows, dorsocentrals $2+3$, intra-alars $1+2$, supra-alars $1+2$, postpronotals 2 . Anepimeron bare. Scutellum greyish dusted, with a pair of strong basal scutellar and a pair of strong apical scutellar setae.

Legs. All coxae black, greyish dusted. All femora black, except $1 / 4$ or $1 / 5$ of yellow apex, greyish dusted. All tibiae and tarsi yellow. Fore femur covered with whitish and blackish hairs, without conspicuous setae. Fore tibia with a row of posterodorsal setae, with 1-2 dorsal, 1-2 posterior, 1 preapical anterodorsal, 1 apical posterodorsal, 1 apical posterior setae. Mid femur with a row of anterodorsal setae, with 1 preapical posterodorsal and 1 preapical posterior setae. Mid tibia with 2 anterodorsal, 2 posterodorsal, $0-1$ anteroventral setae, also with a ring of apicals. Hind femur with a row of dorsal/ anterodorsal setae. Hind tibia with 2-3 posterodorsal, 2-3 anterodorsal, 1 anteroventral, 1 preapical dorsal, 1 preapical anterodorsal, and 2 apical anteroventral setae.

Wing clear; crossveins r-m and d-m not darkened. 
Abdomen black, greyish dusted, covered with whitish hairs. Male sternite 4 about 1.5 times as long as wide (Fig. 15). Male sternite 5 with little curved internally conical lateral lobes, each with external long setae (Fig. 46). Epandrium, cercal plate and surstyli as in Figs 47, 48. Aedeagus with curved apical part, with acrophallus and paraphallus forming ring (Fig. 130).

Female unknown.

DISTRIBUTION. Russia: Buryatia.

\section{Scathophaga calida (Haliday in Curtis, 1832) Figs 16, 49-51.}

calida Haliday in Curtis, 1832: 405 (Scatophaga). Type-locality: "Ireland".

rudis Haliday in Curtis, 1832: 405 (Scatophaga). Type-locality: "Ireland".

villipes Zetterstedt, 1846: 1977 (Scatomyza). Type-localities: "inferalpinis maritimis Finmarkiae occidentalis Norwegiae ... ad Bossekop.” (Finland, Norway).

REMARK. The species was registered for Russia from coasts of Kola Peninsula and White Sea [Gorodkov, 1970: 451, 1986: 30].

MATERIAL EXAMINED. Arkhangelsk Oblast: Solovetskie Islands, Bol'shoy Solovetsky I. (65.090 $\left.\mathrm{N} 35.639^{\circ} \mathrm{E}\right), 17$. VIII. 1963 (1 9 , ZISP); Murmansk Oblast: Aleksandrovsk [= Polyarny $\left(69.198^{\circ} \mathrm{N} 33.456^{\circ} \mathrm{E}\right), 14 . \mathrm{VI} .1921$, Zhelokhovtsev (3 $\sigma^{7} \sigma^{7}, 3$ of, ZMUM); same place, 14-15.IX.1928, Cheburova (11 $\sigma^{7} \sigma^{\top}, 8$ 우, ZISP); Dal'nie Zelentsy $\left(69.117^{\circ} \mathrm{N} 36.065^{\circ} \mathrm{E}\right), 6$.VIII.1981, Gorod-

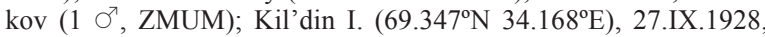

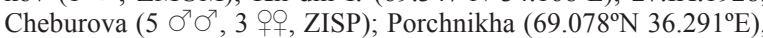

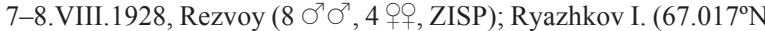
$\left.32.556^{\circ} \mathrm{E}\right)$, 19.VII.1981, Komarova (1 +, ZMUM); same place, 5.VII.1981, Oskol'sky (1 +, ZMUM); same place,12.VI.1992, E.V. Shutova (2 $\sigma^{7} \sigma^{7}, 1$ O ZMUM); Sosnovka $\left(66.507^{\circ} \mathrm{N} 40.583^{\circ} \mathrm{E}\right)$, 23.VIII.1981, Gorodkov (10 ऊ $\sigma^{\top}$, ZISP); Teriberka $\left(69.164^{\circ} \mathrm{N}\right.$ $\left.35.14^{\circ} \mathrm{E}\right)$, 3.VII.2008, V. Semenov (2 $\sigma^{\top} \sigma^{7}$, ZMUM); Yagodny I. $\left(66.769^{\circ} \mathrm{N} 32.069^{\circ} \mathrm{E}\right), 12 . \mathrm{VI} .1992$, E.V. Shutova (16 $\sigma^{\top} \sigma^{\top}, 1$, ZMUM).

DESCRIPTION. Male. Female. Body-length 4.8$8.2 \mathrm{~mm}$.

Head. Frontal vitta reddish-orange, with delicate whitish reflection; fronto-orbital plate black, greyish dusted. Ocellar triangle black. Face, parafacial and gena reddish-orange, with whitish reflection. Postcranium black. 3-5 orbital and 5-7 frontal setae present. Scapus and pedicel dark reddish-orange. Postpedicel black, approximately 2 times as long as wide. Arista bare. Palpus yellow.

Thorax black, densely greyish dusted, with dark stripes and fuzzy spots on scutum and scutellum. Acrostichals in two rows, dorsocentrals (3-4)+4, intra-alars $1+3$, supra-alars $1+2$, postpronotals 2 . Anepimeron covered with hairs. Scutellum black, greyish dusted, with a pair of strong basal scutellar and a pair of strong apical scutellar setae, also with a pair of discal setulae.

Legs densely greyish dusted. All coxae black; all femora usually black, except yellow apex and base or black completely; all tibiae and tarsi yellow. Male legs covered with dense, long hairs, as a rule, without conspicuous setae, except one posteroventral seta on mid tibia and apical setae on mid and hind tibiae. Female: fore femur also without conspicuous setae; fore tibia with 1 anterodorsal, 1 dorsal, 1 posterior, 1 preapical dorsal, and 1 posterior apical setae; mid femur with irregular row of anterodorsal setae, also with 1 preapical posterior and 1 preapical posterodorsal setae; mid tibia with 2 posterodorsal, 2 anterodorsal, 2 anteroventral, 1 posteroventral, 1 ventral, 1 posterior setae and a ring of apicals; hind femur with rows of anterodorsal and anteroventral setae; hind tibia with 2-4 anterodorsal, 2-4 posterodorsal, 1 preapical dorsal, 1 preapical anterodorsal, 1 apical anterior, 1 apical anteroventral and 1 apical ventral setae.

Wing tinged with brownish; crossveins r-m and d-m not darkened.

Abdomen black, densely greyish dusted, covered with dense hairs in male; in female tergites 2-6 with black marginal setulae. Male sternite 4 about twice as long as wide (Fig. 16); sternite 5 with moderate long and wide lateral lobes (Fig. 49). Epandrium, cercal plate and surstyli as in Figs 50, 51.

DISTRIBUTION. Russia: coasts of Kola Peninsula and White Sea. - Europe (Finland, Great Britain, Iceland, Ireland, Norway, Sweden).

\section{Scathophaga cordylurina (Holmgren, 1883)} Figs 17, 52-54.

cordylurina Holmgren, 1883: 173 (Scatomyza). Type-locality: Waigatsch. Schabarowa (Russia: Vaigach Peninsula, Khabarovo). longinqua Becker, 1915: 66 (Scopeuma). Type-locality: "Tundra des Fl. Kara" (Russia: Tyumen' Oblast).

REMARK. The species was recorded in Russia from north of european part (Yugorsky Peninsula, Kolguev I., Vaygach I.) [Holmgren, 1883: 173; Gorodkov, 1970: 451, 1986: 30], Siberia (Tyumen' Oblast, Taimyr Peninsula) [Becker, 1915: 66 (as S. longinqua); Ozerov, Barkalov, 2014: 563], Yakutia [Engelmark, 1999: 159], Far East [Gorodkov, 1986: 30 (without specifying the place of collecting); Ozerov \& Krivosheina, 2014: 216].

MATERIAL EXAMINED. Arkhangelsk Oblast: Amderma $\left(69.756^{\circ} \mathrm{N} 61.661^{\circ} \mathrm{E}\right), 10-12$. VIII.1961, Gorodkov $\left(5 \mathrm{O}^{7} \mathrm{O}^{\top}, 2\right.$ 우, ZISP); Kara [= Ust'-Kara $\left(69.246^{\circ} \mathrm{N} 64.923^{\circ} \mathrm{E}\right)$ ], 10-11.VIII.1961, Gorodkov (6 $0^{7} \sigma^{7}, 2$ 웅, ZISP); Kolguev I., Bugrino $\left(68.782^{\circ} \mathrm{N}\right.$

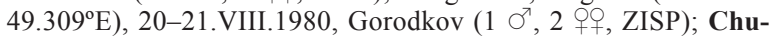
kotka: Iul'tin $\left(67.866^{\circ} \mathrm{N} 178.733^{\circ} \mathrm{W}\right), 21$.VII.1963, Gorodkov (1 $\mathrm{O}^{7}, 2$ 우, ZISP); Pevek $\left(69.702^{\circ} \mathrm{N} 170.298^{\circ} \mathrm{E}\right), 3$.VII.1963, Gorodkov ( $6 \mathrm{O}^{7} \mathrm{O}^{7}, 3$ O $\mathrm{+}$, ZISP); Vrangel I., Mts Somnitel'nye $\left(71.0^{\circ} \mathrm{N}\right.$, $\left.179.533^{\circ} \mathrm{E}\right)$, 14.VII.-15.VIII.2006, O. Khruleva (1 $0^{7}, 1$, ZMUM); Krasnoyarsk Krai: Agapa $\left(71.421^{\circ} \mathrm{N} 89.2512^{\circ} \mathrm{E}\right)$, River Pyasina, 15-19.VII.1967, Gorodkov (12 $0^{7} 0^{7}, 7$ 우, ZISP); Dikson $\left(73.508^{\circ} \mathrm{N} 80.5297^{\circ} \mathrm{E}\right), 8$.VIII.1967, Gorodkov (2 $\mathrm{O}^{7} \mathrm{O}^{7}, 1$ +, ZISP); Dikson I. (73.505 $\left.{ }^{\circ} \mathrm{N} 80.352^{\circ} \mathrm{E}\right), 2$ 27.VII.1948, Korotkevich (22 $\sigma^{7} \sigma^{7}, 17$ OO, ZISP); Dudinka $\left(69.404^{\circ} \mathrm{N} 86.182^{\circ} \mathrm{E}\right)$, 2.VII.1967, Gorodkov (8 $0^{7} \sigma^{7}, 4$ Oᄋ, ZISP); same place, 2629.VII.2011, N. Vikhrev (1 $\bigcirc^{7}$, ZMUM); Igarka $\left(67.467^{\circ} \mathrm{N}\right.$ $\left.86.603^{\circ} \mathrm{E}\right), 26$.VII.1966, V. Sychevskaya (2 90, ZMUM); Yakutia: env. of Ystannakh-Khocho Vill. (ca. $72.58^{\circ} \mathrm{N}, 121.42^{\circ} \mathrm{E}$ ), 20.VIII.2010, A. Yadrenkin ( $3 \bigcirc^{7} \sigma^{7}, 1$ \% , ZMUM); Nizhneyansk $\left(71.441^{\circ} \mathrm{N} 136.1355^{\circ} \mathrm{E}\right), 31$ VII.1974, Gorodkov (3 $0^{7} \sigma^{7}$, ZISP); Saskylakh env. $\left(71.966^{\circ} \mathrm{N} 114.094^{\circ} \mathrm{E}\right)$, 9.VIII.1988, Gorodkov (12 $\bigcirc^{\top} \sigma^{7}, 6$ क 9 , ZISP); Tiksi env. $\left(71.635^{\circ} \mathrm{N} 128.857^{\circ} \mathrm{E}\right), 9$.VII. and 11.VIII.1957, Gorodkov (20 $\mathrm{O}^{7} \mathrm{O}^{7}, 8$ 우, ZISP); UryungKhaya $\left(72.811^{\circ} \mathrm{N} 113.232^{\circ} \mathrm{E}\right), 115 \mathrm{~km}$ ENE, 8.VIII.1988, Gorodkov $\left(26 \bigcirc^{7} 0^{7}, 19\right.$ of , ZISP). See also Ozerov \& Krivosheina [2014: 218] and Ozerov \& Barkalov [2014: 563].

DESCRIPTION. Male. Female. Body-length 5.6- 
Head. Frontal vitta reddish-orange, with delicate whitish reflection; fronto-orbital plate black, delicate greyish dusted. Face, parafacial and gena reddishorange or blackish, with whitish reflection. Postcranium black. 3 orbital and 5-7 frontal setae present. Antenna black. Postpedicel approximately 2 times as long as wide. Arista pubescent, the longest hairs approximately equal to $1 / 3-1 / 2$ width of postpedicel. Palpus yellow.

Thorax black, densely greyish dusted, with dark stripes and fuzzy spots on scutum and scutellum. Acrostichals not differentiated from the other hairs on scutum, $2+3$ hair-like dorsocentrals, intra-alars $1+2$, supra-alars $1+2$, postpronotals 1-2. Anepimeron bare. Scutellum black, greyish dusted, with a pair of strong basal scutellar and a pair of strong apical scutellar setae, also with 1-3 pairs of lateral scutellar setae.

Legs greyish dusted or shiny (rarely). All coxae black; all femora from almost completely yellow to black completely; all tibiae and tarsi brownish or dark brown. Male femora covered with dense, long hairs, without conspicuous setae. Female fore femur without conspicuous setae. Fore tibia with 2-3 dorsal, 0-1 posterior, 1 preapical dorsal, and 1 posterior apical setae. Mid femur with a row of anterodorsal setae in female, also with 1 preapical posterior and 1 preapical posterodorsal setae in both sexes. Mid tibia with 1 posterodorsal, 1 anterodorsal setae and a ring of apicals. Female hind femur with a row of anterodorsal setae or setulae. Hind tibia with 2 anterodorsal, 2 posterodorsal, 1 preapical dorsal, 1 preapical anterodorsal, 1 apical anteroventral setae.

Wing tinged with brownish; crossveins r-m and d-m not darkened.

Abdomen black, densely greyish dusted, covered with dense hairs in male, less dense and shorter in female; female tergites 2-6 without strong marginal setae. Male sternite 4 wider than long (Fig. 17); sternite 5 wide, without lateral lobes, but with small apical median processes (Fig. 52). Epandrium, cercal plate and surstyli as in Figs 53, 54.

DISTRIBUTION. Russia: north from Kola Peninsula to Chukotka.

\section{Scathophaga crinita (Coquillett, 1901)} Figs 18, 55-57.

crinita Coquillett, 1901: 612 (Scatophaga). Type-locality: "Bering Island" (Russia: Commander Islands).

REMARK. This species was recorded in Russia for Far East (coasts of Chukotka and Kamchatka, Commander Islands) [Coquillett, 1901: 612; Gorodkov, 1986: 30; Ozerov, Krivosheina, 2014: 216].

MATERIAL EXAMINED. Chukotka: $5 \mathrm{~km}$ N of Egvekinot $\left(66.395^{\circ} \mathrm{N} 179.132^{\circ} \mathrm{W}\right), 26 . \mathrm{VII} .1963$, Gorodkov $\left(6 \mathrm{O}^{7} \mathrm{O}^{7}, 5\right.$ 우, ZISP); Goryachie klyuchi [? Lorino: $65.503^{\circ} \mathrm{N} 171.704^{\circ} \mathrm{W}$ ], 10.VIII.1960, Kononov (5 $\sigma^{7} \sigma^{7}, 5$ 우, ZISP); same place, 7.VIII.1963, Gorodkov (4 $\sigma^{7} \sigma^{7}, 7$ of , ZISP); Pevek $\left(69.702^{\circ} \mathrm{N}\right.$ $\left.170.298^{\circ} \mathrm{E}\right)$, 25.VIII.1970, B. Petrova (1 $0^{\top}, 1$ ㅇ, ZISP); Tkachen Bay $\left(64.417^{\circ} \mathrm{N} 172.818^{\circ} \mathrm{W}\right), 25-26 . V I I I .1938$, Rozanov $\left(5 \mathrm{O}^{\top} \mathrm{O}^{7}, 1\right.$ o, ZISP); Ureliki $\left(64.402^{\circ} \mathrm{N} 173.210^{\circ} \mathrm{W}\right), 3-6 . V I I I .1963$, Gorodkov (1 $0^{7}, 4$ 우, ZISP); Kamchatka Krai: Apuka env. $\left(60.443^{\circ} \mathrm{N}\right.$ $\left.169.602^{\circ} \mathrm{E}\right), 1-2$. and 30.VII.1959, Gorodkov ( $0^{7} 0^{7}, 6$ 우, ZISP);
Commander Islands, Mednyy I. (54.709 $\left.{ }^{\circ} \mathrm{N} 167.709^{\circ} \mathrm{E}\right)$, 1.VII.1958, Violovich (6 $\sigma^{7} \sigma^{7}, 2$, 2 , ZISP); Commander Islands, Nikol'skoe $\left(55.1961^{\circ} \mathrm{N} 165.998^{\circ} \mathrm{E}\right), 4,6$ and 14.IX.1959, Gorodkov (13 $\sigma^{7} \mathrm{O}^{7}$, 2 오, ZISP); Commander Islands, Preobrazhenskoe $\left(54.790^{\circ} \mathrm{N}\right.$ 167.578 E), 6.VII.1911, Suvorov (1 $0^{7}$, ZISP); same place, 5 and 6.IX.1959, Gorodkov (4 $\bigcirc^{7} \sigma^{7}, 1$, ZISP). See also Ozerov \& Krivosheina [2014: 216]

DESCRIPTION. Male. Female. Body-length 5.8$10.0 \mathrm{~mm}$.

Male thorax, abdomen and legs covered with dense, furry, crinkly, yellow hairs. Female has yellow hairs shorter; abdomen covered with yellow hairs on ventral surface only.

Head. Frontal vitta reddish-yellow, with delicate whitish reflection; fronto-orbital plate black, greyish dusted. Face, parafacial and gena reddish, with delicate whitish reflection. Postcranium black. 3 orbital and 5-6 frontal setae present. Antenna black. Postpedicel approximately 1.5 times as long as wide. Arista bare. Palpus yellow.

Thorax black, greyish dusted. Acrostichals in two rows, dorsocentrals $(4-5)+(5-6)$, intra-alars $1+(2-4)$, supra-alars $(1-2)+(2-4)$, postpronotals 2 . Anepimeron bare. Scutellum black, greyish dusted, with a pair of strong basal scutellar and a pair of strong apical scutellar setae.

Legs black, shiny or subshiny. All femora covered with hairs, but without conspicuous setae. Fore tibia with rows of dorsal and posterodorsal setae. Mid tibia with 2-3 posterodorsal, 1-2 anterodorsal setae and a ring of apicals in both sexes, additionally with 1 ventral seta in female. Hind tibia with a comb of short stong apical setae on anterior surface in both sexes, additionally with rows of thin posterodorsal and anterodorsal setae in female.

Wing tinged with brownish; crossveins r-m and dmcu not darkened.

Abdomen black, delicately greyish dusted. Male sternite 4 approximately 2 times as long as wide (Fig. 18 ); sternite 5 with short lateral lobes, without median processes (Fig. 55). Epandrium, cercal plate and surstyli as in Figs 56, 57.

DISTRIBUTION. Russia: coasts along of Chukchi Sea (Pevek) to Bering Sea before Olutorsky Bay, Commander Islands. - North America.

\section{Scathophaga dasythrix (Becker, 1894)} Figs 19, 58-60.

dasythrix Becker, 1894: 173 (Scatophaga). Type-locality: Medny I., Commander Islands (Russia).

REMARK. This species was recorded in Russia from Far East (including Chukotka, Kamchatka, and Kuril Islands) [Becker, 1894: 173; Gorodkov, 1986: 30 (without specifying the place of collecting); Ozerov, Krivosheina, 2014: 217].

MATERIAL EXAMINED. Chukotka: Provideniya $\left(64.427^{\circ} \mathrm{N}\right.$ $\left.173.225^{\circ} \mathrm{W}\right), 2$.VIII.1963, Gorodkov (1 $\mathrm{O}^{7}$, ZISP); Ureliki $\left(64.400^{\circ} \mathrm{N}\right.$ $173.214^{\circ} \mathrm{W}$ ), 6.VIII.1963, Gorodkov (1 $0^{7}$, ZISP); Kamchatka Krai: Apuka $\left(60.444^{\circ} \mathrm{N} 169.601^{\circ} \mathrm{E}\right), 10$ and 31.VII., 2 and 4.VIII.1959, Gorodkov (4 $\sigma^{7} \sigma^{7}, 3$ 우, ZISP); Commander Isands, Bering I. $\left(55.065^{\circ} \mathrm{N} 166.225^{\circ} \mathrm{E}\right), 5-7 . \mathrm{VIII} .1931$, I. Iogansen $\left(5 \mathrm{O}^{7} \mathrm{O}^{7}, 6 \mathrm{OQ}\right.$, ZISP); Commander Isands, Mednyy I. (54.709 $\left.\mathrm{N} 167.709^{\circ} \mathrm{E}\right)$, 1.VII.1958, Violovich (1 9 , ZISP); Commander Isands, Nikol'skoe 


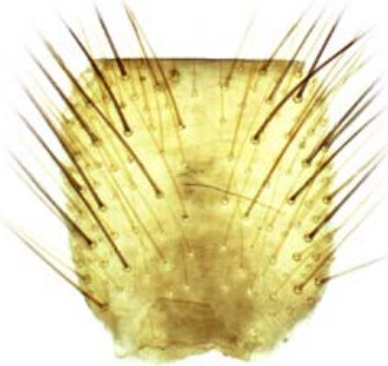

14

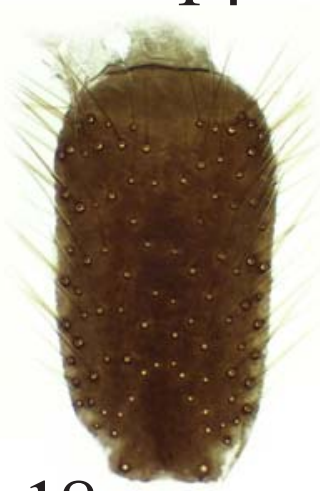

18
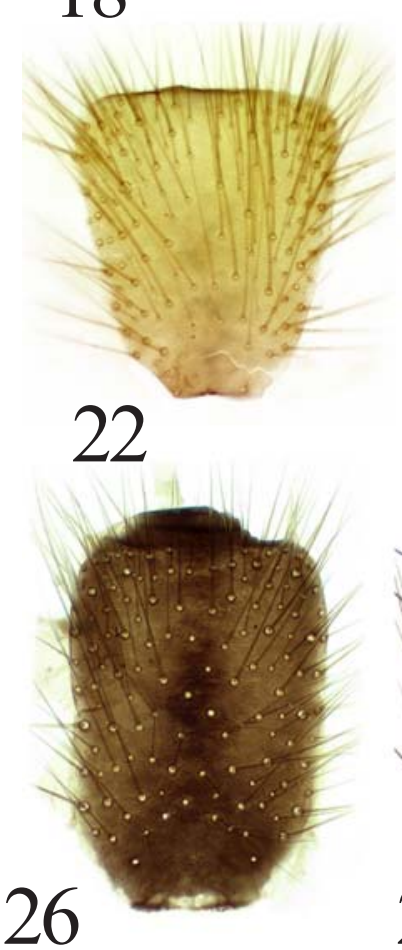

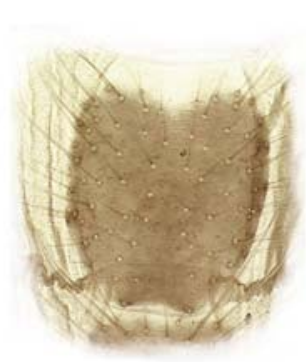

15

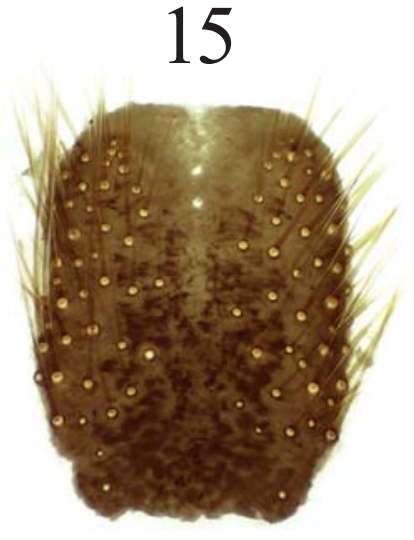

19
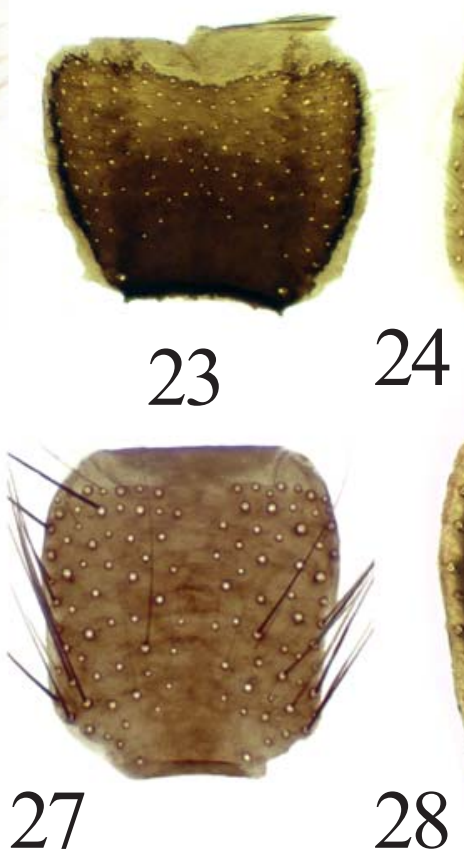

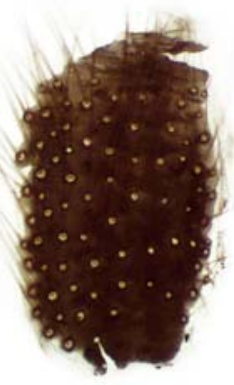

16

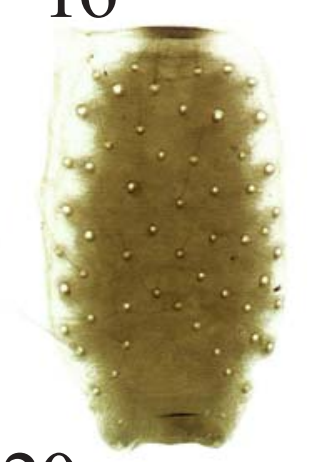

20

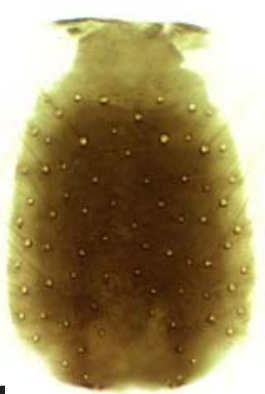

24

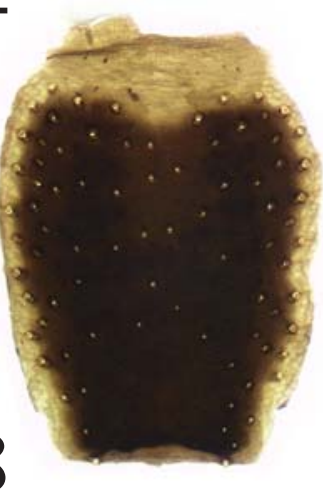

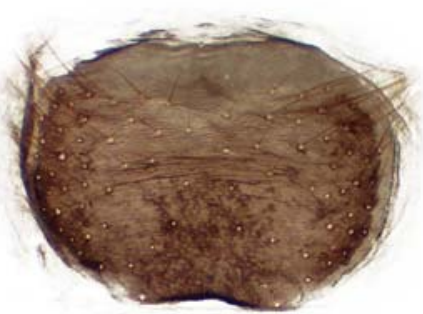

17
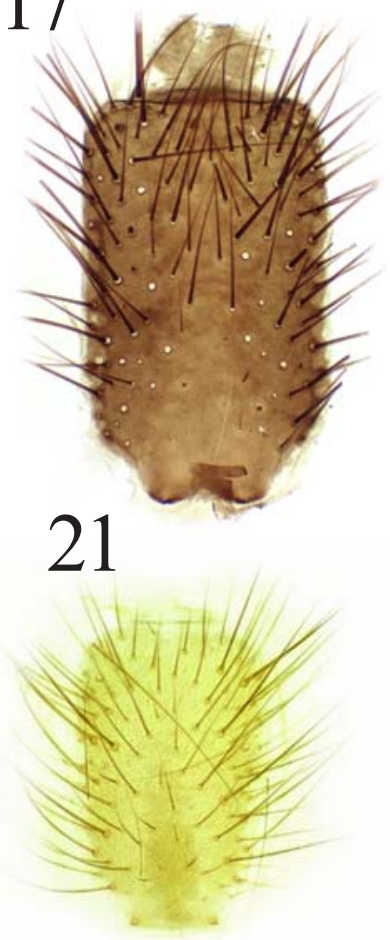

25

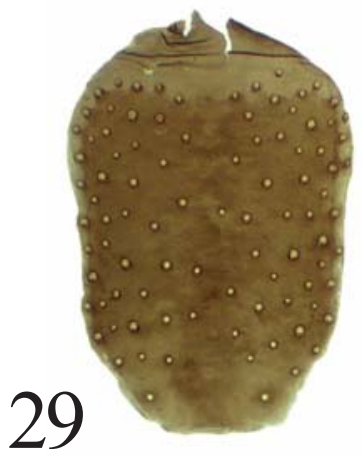

Figs 14-29. Male sternite 4 of Scathophaga spp.: $14-$ S. apicalis (Curtis); 15 - S. buryatica Ozerov et Krivosheina; 16 - S. calida (Haliday); 17 - S. cordylurina (Holmgren); $18-$ S. crinita (Coquillett); $19-$ S. dasythrix (Becker); $20-S$. decipiens (Haliday); 21 $S$. exalata Ozerov; $22-S$. furcata (Say); $23-S$. incola (Becker); $24-$ S. incompleta Ozerov et Krivosheina; $25-S$. inquinata (Meigen); 26 - S. intermedia (Walker); 27 - S. karelica Ozerov; 28 - S. lapponica (Ringdahl); 29 - S. litorea (Fallén). 15 - after Ozerov \& Krivosheina, 2019, fig. 1; 20, 24 - after Ozerov \& Krivosheina, 2020, figs 3, 9; 27 — after Ozerov, 2013 , fig.13.

Рис. 14-29. Стернит 4 самца Scathophaga spp.: 14 - S. apicalis (Curtis); 15 - S. buryatica Ozerov et Krivosheina; 16 - S. calida (Haliday); 17 - S. cordylurina (Holmgren); $18-$ S. crinita (Coquillett); $19-$ S. dasythrix (Becker); $20-S$. decipiens (Haliday); 21 S. exalata Ozerov; $22-S$. furcata (Say); $23-$ S. incola (Becker); $24-S$. incompleta Ozerov et Krivosheina; $25-S$. inquinata (Meigen); $26-S$. intermedia (Walker); $27-$ S. karelica Ozerov; $28-$ S. lapponica (Ringdahl); $29-$ S. litorea (Fallén). $15-$ по Ozerov \& Krivosheina, 2019, fig. 1; 20, 24 — по Ozerov \& Krivosheina, 2020, figs 3, 9; 27 — по Ozerov, 2013 , fig.13. 

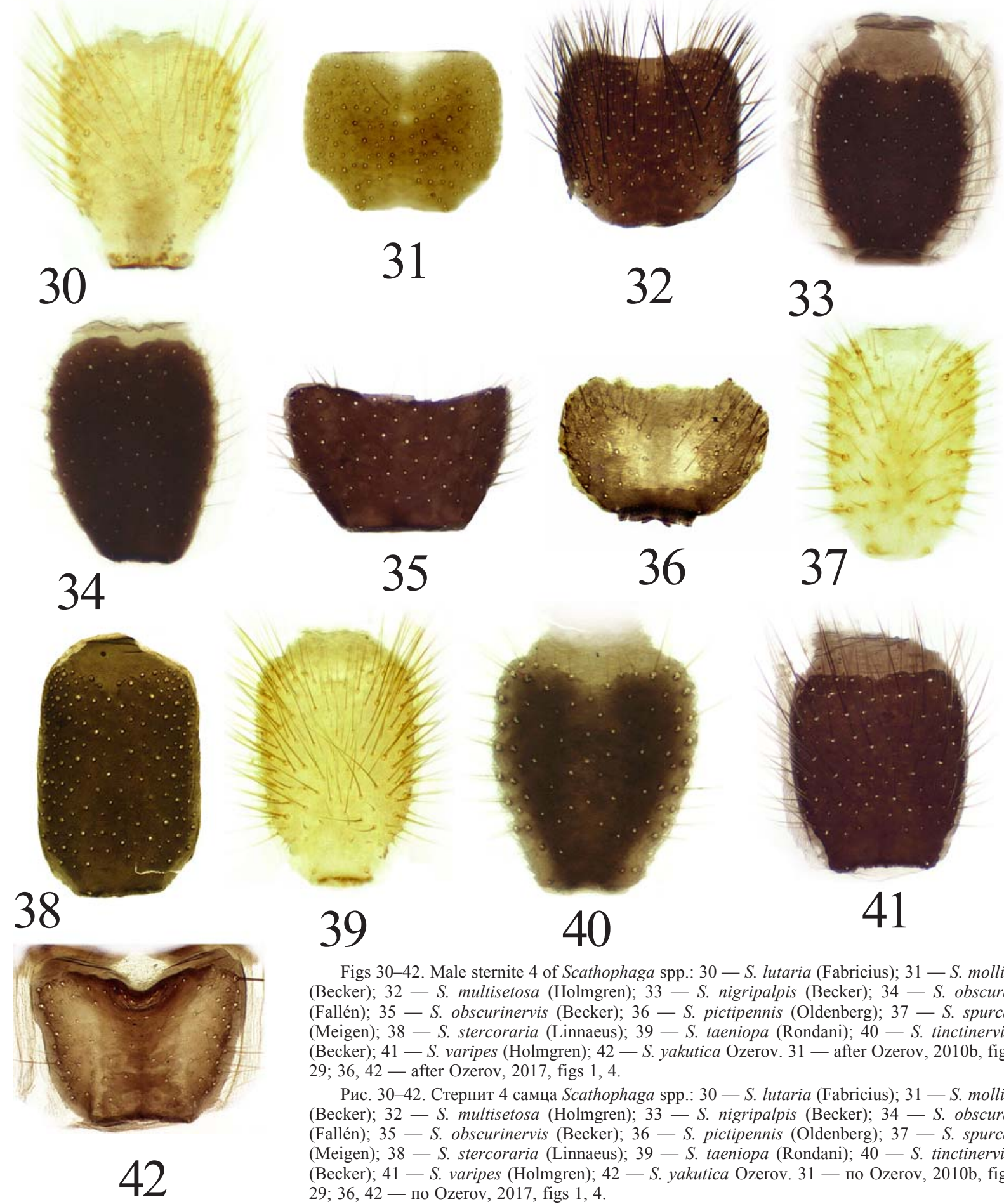

33
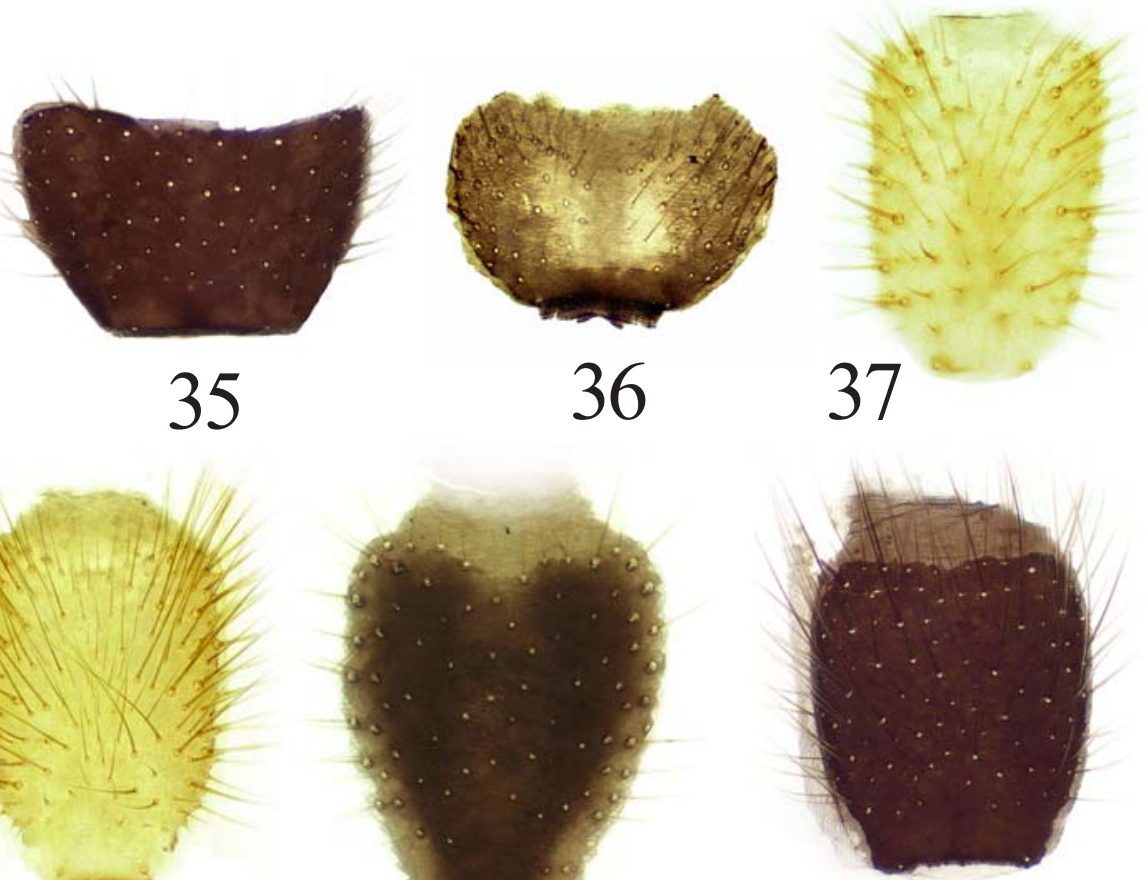

41
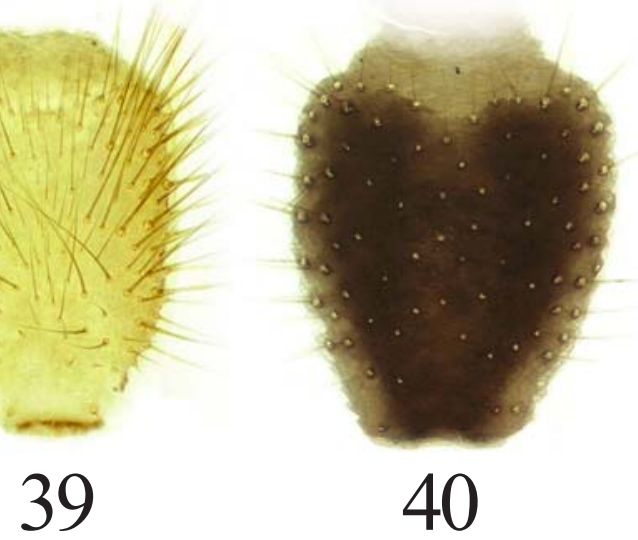

Figs 30-42. Male sternite 4 of Scathophaga spp.: $30-$ S. lutaria (Fabricius); $31-$ S. mollis (Becker); $32-S$. multisetosa (Holmgren); $33-$ S. nigripalpis (Becker); $34-$ S. obscura (Fallén); $35-$ S. obscurinervis (Becker); $36-$ S. pictipennis (Oldenberg); $37-$ S. spurca (Meigen); $38-$ S. stercoraria (Linnaeus); $39-$ S. taeniopa (Rondani); $40-$ S. tinctinervis (Becker); $41-S$. varipes (Holmgren); $42-S$. yakutica Ozerov. 31 - after Ozerov, 2010b, fig. 29; 36, 42 - after Ozerov, 2017, figs 1, 4.

Рис. 30-42. Стернит 4 самца Scathophaga spp.: $30-$ S. lutaria (Fabricius); $31-$ S. mollis (Becker); $32-S$. multisetosa (Holmgren); $33-$ S. nigripalpis (Becker); $34-$ S. obscura (Fallén); $35-$ S. obscurinervis (Becker); $36-$ S. pictipennis (Oldenberg); $37-$ S. spurca (Meigen); $38-$ S. stercoraria (Linnaeus); $39-$ S. taeniopa (Rondani); $40-$ S. tinctinervis (Becker); $41-S$. varipes (Holmgren); $42-$ S. yakutica Ozerov. 31 - по Ozerov, 2010b, fig. 29; 36, 42 - по Ozerov, 2017, figs 1, 4.

env. $\left(55.196^{\circ} \mathrm{N} 165.998^{\circ} \mathrm{E}\right)$, 4.IX.1959, Gorodkov (3 $0^{7} \sigma^{7}, 1$ ㅇ, ZISP); Commander Isands, Preobrazhenskoe $\left(54.790^{\circ} \mathrm{N} 167.578^{\circ} \mathrm{E}\right)$, 1.VI.1910, 4 and 5.VII.1911, Suvorov (6 $\sigma^{7} \sigma^{7}, 10$ 우, ZISP); same place, 6 and 7.IX.1959, Gorodkov (3 $\sigma^{7} \sigma^{7}, 1$ ㅇ, ZISP); Korf $\left(60.376^{\circ} \mathrm{N} 166.023^{\circ} \mathrm{E}\right), 22$. VI.1959, Gorodkov (1 $\circ$, ZISP); Utashud I. $\left(51.507^{\circ} \mathrm{N} 157.696^{\circ} \mathrm{E}\right), 9$. VII.1958, Violovich $\left(1 \mathrm{O}^{\top}\right.$, ZISP). See also Ozerov \& Krivosheina [2014: 217].

DESCRIPTION. Male. Female. Body-length 6.2$9.8 \mathrm{~mm}$. Male thorax, abdomen and legs covered with dense, furry, crinkly, blackish or yellowish hairs. Female has hairs shorter; abdomen covered with hairs on ventral surface only.

Head. Frontal vitta blackish, only anteriorly dark reddish, with delicate whitish reflection; fronto-orbital plate black, greyish dusted. Face, parafacial and gena reddish, with delicate whitish reflection. Postcranium black. 3 orbital and 6-7 frontal setae present. Antenna 
black. Postpedicel approximately 1.5 times as long as wide. Arista bare. Palpus yellow.

Thorax black, densely greyish dusted. Scutum with setae only slightly differentiated from the other long hairs on scutum. Anepimeron with several hairs. Scutellum black, greyish dusted, with 4-6 pairs of setae along margin.

Legs black, densely greyish dusted. Male legs covered with long hairs, well visible apical setae on mid and hind tibiae only. Female: all femora covered with hairs, but without conspicuous setae; fore tibia with rows of dorsal and posterodorsal setae; mid tibia with 2-3 posterodorsal, 2-3 anterodorsal, 1 ventral setae and a ring of apicals; hind tibia with rows of thin posterodorsal and anterodorsal setae and a comb of short stong apical setae on anterior surface.

Wing tinged with brownish; crossveins r-m and dmcu not darkened.

Abdomen black, densely greyish dusted. Male sternite 4 approximately 2 times as long as wide (Fig. 19), Male sternite 5, epandrium, cercal plate and surstyli as in Figs 58-60.

DISTRIBUTION. Russia: Pacific coast of Bering Strait south to Olutorsky Bay, Commander Islands; north Kuril Islands. - North America.

\section{Scathophaga decipiens (Haliday in Curtis, 1832) Figs 20, 61-63, 131.}

decipiens Haliday in Curtis, 1832: 266 (Scatophaga). Typelocalities: "Ireland,... England".

fluvialis Rondani, 1866: 28, 29; 1867: 113 (Scatina). Typelocality: not given [Italia: Parma].

REMARKS. This species was recorded in Russia from Crimea and Rostov Oblast by Ozerov \& Krivosheina [2020: 228]. Noted from south of European part by Gorodkov [1970: 453, 1986: 31] without specifying the place of collecting.

MATERIAL EXAMINED. Crimea: Kerch env. $\left(45.353^{\circ} \mathrm{N}\right.$ $\left.36.445^{\circ} \mathrm{E}\right), 10$ and 30.VI.1901, 14.V.1903, Kirichenko (1 ${ }^{\top}, 2$ 우, ZMUM). See also Ozerov \& Krivosheina [2020: 228]. $6.3 \mathrm{~mm}$.

DESCRIPTION. Male. Female. Body-length 5.8-

Head. Frontal vitta yellow or yellow-reddish, with delicate whitish reflection; fronto-orbital plate blackish, densely greyish dusted. Face, parafacial and gena yellow, with whitish reflection. Postcranium blackish. 3 orbital and 3-4 frontal setae present. Antenna black; postpedicel about twice as long as wide. Arista bare. Palpus yellow.

Thorax black, densely greyish dusted. Acrostichals not differentiated from the other hairs on scutum, dorsocentrals $2+3$, intra-alars $1+2$, supra-alars $1+2$, postpronotals 2. Anepimeron bare. Scutellum greyish dusted, with a pair of strong basal scutellar and a pair of strong apical scutellar setae.

Legs. All coxae black, greyish dusted. All femora greyish dusted, black, except yellow apex. All tibiae and tarsi yellow. Fore femur covered with whitish hairs, with 3-4 dorsal setae or setulae in apical third. Fore tibia with 2 dorsal, 2 posterior, 2-4 posterodorsal, 1 preapical anterodorsal, and 1 posterior apical setae. Mid femur with a row of anterior setae, 1 preapical posterior and 1 preapical posterodorsal setae. Mid tibia with 1-2 anterodorsal, 1-2 posterodorsal, $0-1$ anteroventral, $0-1$ ventral setae, also with a ring of apicals. Hind femur with a row of anterodorsal setae. Hind tibia with 2 posterodorsal, 3 anterodorsal, 1 preapical dorsal, 1 preapical anterodorsal and 1 apical anteroventral setae.

Wing clear; crossveins $\mathrm{r}-\mathrm{m}$ and $\mathrm{d}-\mathrm{m}$ not darkened.

Abdomen black, densely pale grey dusted, covered with short whitish hairs. Tergites $2-5$ in male and tergiters $2-6$ in female each with a row of marginal setae. Male sternite 4 about twice as long as wide (Fig. 20). Male sternite 5 with long narrow lobes (Fig. 61). Cercal plate longer than surstyli (Figs 62, 63). Aedeagus as in Fig.131.

DISTRIBUTION. Russia: Crimea and Rostov Oblast. - Europe, North Africa [Šifner, 2008: 157, 158].

\section{Scathophaga exalata Ozerov, 1996} Figs 7, 21, 64-66.

exalata Ozerov, 1996: 2 (Scathophaga). Type-locality: Raikoke I. (48 $\left.17^{\prime} 86^{\prime \prime} \mathrm{N}, 153^{\circ} 15^{\prime} 64^{\prime \prime} \mathrm{E}\right)$ (Russia: Kuril Islands).

REMARK. This species known from Kuril Islands only (Raikoke I., Chirinkotan I., Ekarma I., Yankicha I.) [Ozerov, 1996: 2].

MATERIAL EXAMINED. Sakhalin Oblast: Yankicha I. $\left(47.517^{\circ} \mathrm{N} 152.811^{\circ} \mathrm{E}\right), 16$. VII.1958, Violovich (1 $\sigma^{7}, 1$ \% , ZISP). See also Ozerov [1996: 2].

DESCRIPTION. Male. Female. Length of body 6.5$12.0 \mathrm{~mm}$.

Thorax, abdomen and legs of males covered with dense, long, brown hairs. Females has hairs less dense and shorter.

Head. Frontal vitta reddish-orange, with delicate whitish reflection; fronto-orbital plate black, greyish dusted. Face and gena reddish-orange, with whitish reflection. Postcranium black. 3 orbital and 5-7 frontal setae present. Scapus and pedicel from dark reddish to black. Postpedicel black, about twice as long as wide. Arista bare. Palpus yellow.

Thorax black, densely grey dusted. Scutum chaetotaxy better seen in females: acrostichals setulose in two rows, dorsocentrals $2+2$, intra-alars $1+2$, supra-alars $1+2$, postpronotals 2 . Anepimeron bare. Scutellum greyish dusted, with a pair of strong basal scutellar and a pair of apical scutellar setulae.

Legs brownish or blackish. Legs of male covered with dense, long hairs; female legs with less dense and shorter hairs. Femora of all legs of both sexes without conspicuous setae. Male fore tibia with 2 very strong posteroventral setae in apical quarter. Female fore tibia with 2-3 dorsal, 2 posterior, 3-4 posteroventral setae (including apical seta). Male mid tibia with 1 anterodorsal, 2 anterior, 1 ventral setae, also with a ring of apicals. Female mid tibia with 3-4 anterior, 3 anterodorsal, 3 anteroventral, 2-3 posterodorsal, 2 posterior, 1 ventral setae, also with a ring of apicals. Male hind tibia with 1 preapical anterodorsal, 1 apical anterior, 1 apical anteroventral, 1 apical ventral setae. Female hind tibia with 3 posterodorsal, 4 anterodorsal, 1 preapical 
dorsal, 1 preapical anterodorsal, 1 apical anterior, 1 apical anteroventral, 1 apical ventral setae.

Wing strongly reduced (Fig. 7).

Abdomen black, delicately greyish dusted, covered with long brownish hairs in male; female tergites 2-6 each with a row of marginal setae. Male sternite 4 about 2 times as long as wide (Fig. 21). Male sternite 5 with broad in base lateral lobes, covered with long hairs (Fig. 64). Epandrium, cercal plate and surstyli as in Figs 65, 66.

DISTRIBUTION. Russia: Kuril Islands.

Scathophaga furcata (Say, 1823)

Figs 3, 22, 67-69.

(USA)

furcata Say, 1823: 98 (Pyropa). Type-locality: "Missouri"

squalida Meigen, 1826: 252 (Scatophaga). Type-locality: not given ("hiesiger Gegend", ?Aachen).

fuscinervis Zetterstedt, 1838: 722 (Scatomyza). Type-localities: "Lapponia Tornensi... Wittangi... Juckasjervi... Tornetrask... Dowre... Kloeffjoefjellet in Herjeådalen...” (Sweden, Norway).

fuscinervis Zetterstedt, 1838: 733 (Cordylura). Type-locality: "ad Umenaes... (Lappon.)" (Sweden).

semiatra de Meijere, 1907: 181 (Scathophaga). Type-locality: Hilversum (Netherlands).

REMARKS. The data on distribution of this species for Russia was given by Hendel [1930: 2] (Kamchatka), by Gorodkov [1970: 453, 1986: 31] (European part, incl. Kolguev I., Commander and Kuril Islands), by Polevoi [1997: 309] (Karelia), by Engelmark [1999: 158, 159] (arctic zone of European part), by Ovchinnikov [2004: 422] (Yaroslavl' Oblast), by Ozerov \& Barkalov [2014: 563] (Taimyr Peninsula), by Ozerov \& Krivosheina [2014: 217] (Far East), by Bagachanova et al. [2016: 782] (Yakutia), by Ovchinnikov \& Makarova [2016: 217] (Barents Sea, Dolgii I.) and by [MacGowan et al., [2021: 17] (Mordovia).

Gorodkov published a map with the distribution area of this species on the territory of the former USSR and separately on its European part [Gorodkov, 1978: map 20], but without names of the points.

MATERIAL EXAMINED. Altai: "Rodonovy istochnik", 2412 m, Lake Muzdy-Bulak (49.46 ${ }^{\circ}$ N 88.05 $\left.{ }^{\circ} \mathrm{E}\right)$, 5.VII.2008, A. Barkalov ( 1 ․ ISEA); $20 \mathrm{~km} \mathrm{~W}$ of Beltir $\left(49.95^{\circ} \mathrm{N} 87.86^{\circ} \mathrm{E}\right), 2100 \mathrm{~m}, 24$ 25.VI.2014, I. Shamshev (1 9 , ZISP); Chikhachev ridge, in the source of the River Naryn-Gol, $2600 \mathrm{~m}\left(49.81^{\circ} \mathrm{N} 88.55^{\circ} \mathrm{E}\right)$, 18.VII.2009, A. Barkalov (1 $\sigma^{\top}$, ISEA); Kosh-Agach, plato Ukok, $2400 \mathrm{~m}$, surrounding area of Lake Muzdy-Bulak $\left(49.26^{\circ} \mathrm{N}, 87.65^{\circ} \mathrm{E}\right)$, 10-11.VII.2008, A. Barkalov (2 $\sigma^{7} \sigma^{\top}, 3$ oᄋ, ISEA and ZMUM); Kosh-Agach, plato Ukok, $2450 \mathrm{~m}$, Lake Muzdy-Bulak $\left(49.3^{\circ} \mathrm{N}\right.$ $\left.87.65^{\circ} \mathrm{E}\right), 8$.VII.2008, A. Barkalov (1 + , ISEA); Kurayskiy ridge $2500-2700 \mathrm{~m}\left(50.33^{\circ} \mathrm{N} 87.75^{\circ} \mathrm{E}\right)$, tundra, 3.VII.2008, A. Barkalov (1 9 , ISEA); the upper reaches of the River Naryn-Gol, $2520 \mathrm{~m}$, $\left(49.49^{\circ} \mathrm{N} 89.32^{\circ} \mathrm{E}\right), 16-19 . \mathrm{VII} .2009$, V. Sorokina (1 $\mathrm{O}^{\prime}$, ISEA); Ust-Koksa $\left(50.27^{\circ} \mathrm{N} 85.61^{\circ} \mathrm{E}\right), 24 . \mathrm{IX} .2011$, O. Kosterin (1 $\sigma^{7}$, ZMUM); Arkhangelsk Oblast: $70 \mathrm{~km} \mathrm{~N}$ of Nar'yan-Mar (ca $\left.68.204^{\circ} \mathrm{N} 53.627^{\circ} \mathrm{E}\right)$, 18.VIII.1978, Gorodkov (1 $\bigcirc^{\top}, 2$ 우, ZISP); $73 \mathrm{~km}$ NW of Nar'yan-Mar (ca. $\left.67.981^{\circ} \mathrm{N} 51.608^{\circ} \mathrm{E}\right), 3$. VIII.1978, Gorodkov (1 $\sigma^{7}, 1$, ZISP); Arkhangelsk $\left(64.546^{\circ} \mathrm{N} 40.567^{\circ} \mathrm{E}\right)$ 4.VIII.2010, D. Gavryushin (4 $\sigma^{7} \sigma^{7}$, ZMUM); Arkhangelsk $\left(64.562^{\circ} \mathrm{N} 40.547^{\circ} \mathrm{E}\right), 10 . \mathrm{VIII} .1967,25 . \mathrm{VIII} .1981$, Gorodkov (5 $\sigma^{7} \sigma^{7}$, ZISP); Belush'e env. (66.89 $\left.{ }^{\circ} \mathrm{N} 47.61^{\circ} \mathrm{E}\right), 24$.VIII.1978, Gorodkov (5 $\bigcirc^{7} \sigma^{7}$, ZISP); Kanin Nos $\left(68.656^{\circ} \mathrm{N} 43.279^{\circ} \mathrm{E}\right), 17 . \mathrm{VII} .1970$, Gorodkov (18 $\sigma^{7} \sigma^{7}, 12$ 우, ZISP); Kara [= Ust'-Kara $\left(69.246^{\circ} \mathrm{N}\right.$

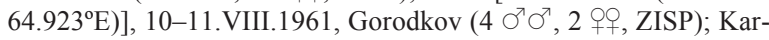

gopol' $\left(61.503^{\circ} \mathrm{N} 38.971^{\circ} \mathrm{E}\right)$, 16.VII.1982, Gorodkov (1 O', ZISP); Khal'mer'yu (ca. $\left.67.550^{\circ} \mathrm{N} 53.962^{\circ} \mathrm{E}\right), 19 . \mathrm{VII} .1962$, Gorodkov (2 $\Im^{7} \sigma^{\top}, 1$ ๆ, ZISP); Kolguev I., Bugrino $\left(68.782^{\circ} \mathrm{N} 49.309^{\circ} \mathrm{E}\right), 20$ 21.VIII.1980, Gorodkov (13 ○ $\sigma^{\top}, 7$ 우, ZISP); Mezen' $\left(65.85^{\circ} \mathrm{N}\right.$ 44.24 $\left.{ }^{\circ} \mathrm{E}\right), 26$ and 27.VIII.1978, Gorodkov (3 $\sigma^{7} \sigma^{7}, 1$ q, ZISP); Nar'yan-Mar $\left(67.631^{\circ} \mathrm{N} 52.985^{\circ} \mathrm{E}\right), 28$.VIII.1970, Gorodkov (5 O' $\sigma^{\prime}$, 3 OO, ZISP); same place, 8, 9 and 13.VII.2008, N. Vikhrev, A.L. Ozerov (12 $\sigma^{\top} \sigma^{\top}, 9$ 90 , ZMUM); Nenetsky Nature Reserve, cordon

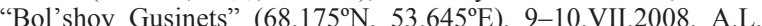
Ozerov (1 $\sigma^{7}$, ZMUM); Nizhnyaya Pesha $\left(66.751^{\circ} \mathrm{N} 47.760^{\circ} \mathrm{E}\right), 22$ and 23.VII.1978, Gorodkov (2 ア $\Im^{\top}, 2$ of, ZISP); River SilovaYakha (ca. $\left.68.558^{\circ} \mathrm{N} 64.817^{\circ} \mathrm{E}\right), 80 \mathrm{~km} \mathrm{~N}$ of Khal'mer'yu, 19.VII.1961, Gorodkov (4 $\sigma^{\top} \sigma^{\top}$, ZISP); Shoyna $\left(67.877^{\circ} \mathrm{N} 44.150^{\circ} \mathrm{E}\right)$, 16.VII.1970, Gorodkov (13 O $^{\top} \sigma^{7}, 9$ OO , ZISP); Solovetsky I. $\left(65.080^{\circ} \mathrm{N}\right.$ $35.686^{\circ} \mathrm{E}$ ), 26.VII.1959, Gorodkov (3 $\sigma^{7} \sigma^{7}, 1$ ㅇ, ZISP); Solvychegovsk env. (61.342 $\left.{ }^{\circ} \mathrm{N} 46.913^{\circ} \mathrm{E}, 61.334^{\circ} \mathrm{N} 46.926^{\circ} \mathrm{E}\right), 2 . I X .1981$, Gorodkov ( $2 \sigma^{\top} \sigma^{\top}, 1$ ㅇ, ZISP); same place, 13 and 17.VIII.2010, D. Gavryushin (1 $\sigma^{7}, 2$ of , ZMUM); the lower reaches of the River Pechora $\left(68.334^{\circ} \mathrm{N} 53.304^{\circ} \mathrm{E}\right), 11$. VII.2008, A.L. Ozerov (1 $\Im^{7}$, ZMUM); Velikovisochnoe $\left(67.255^{\circ} \mathrm{N} 52.032^{\circ} \mathrm{E}\right), 18$.VIII.1978, Gorodkov ( $\Im^{7}, 1$ ㅇ, ZISP); Astrakhan Oblast: Baskunchak salt lake $\left(48.193^{\circ} \mathrm{N} 46.813^{\circ} \mathrm{E}\right), 2-4 . \mathrm{V} .2010$, K. Tomkovich $\left(10^{7}, 1\right.$ 웅, ZMUM); Buryatia: Lake Baykal, Pokoyniki env. $\left(53.713^{\circ} \mathrm{N}\right.$ $\left.109.049^{\circ} \mathrm{E}\right), 27$. VII.1962, Gorodkov (1 $\sigma^{\top}, 1$ \%, ZISP); Mondy env. $\left(51.675^{\circ} \mathrm{N} 100.992^{\circ} \mathrm{E}\right), 28$.VII.1965, Gorodkov (5 $\sigma^{7} \sigma^{7}, 4$ 우, ZISP); Chelyabinsk Oblast: Chelyabinsk, airport $\left(55.302^{\circ} \mathrm{N} 61.502^{\circ} \mathrm{E}\right)$, 5.IX.1988, Gorodkov (1 $\sigma^{7}$, ZISP); Taganay $\left(55.277^{\circ} \mathrm{N} 59.795^{\circ} \mathrm{E}\right)$, 18-24.VII.2008, K. Tomkovich (1 $\sigma^{7}$ ZMUM); Chukotka: 5 km $\mathrm{N}$ of Egvekinot $\left(66.395^{\circ} \mathrm{N} 179.132^{\circ} \mathrm{W}\right), 26 . \mathrm{VII} .1963$, Gorodkov (4 $\bigcirc^{\top} \sigma^{7}, 4$ 우, ZISP); Bilibino $\left(68.058^{\circ} \mathrm{N} 166.446^{\circ} \mathrm{E}\right)$, 5.VII.1971, Gorodkov (3 $\sigma^{7} \sigma^{7}, 2$ 우, ZISP); Komsomol'sky $\left(69.130^{\circ} \mathrm{N}\right.$ $\left.172.735^{\circ} \mathrm{E}\right), 4$ and 7.VII.1963, Gorodkov ( $\sigma^{7}, 1$ +, ZISP); Markovo (ca. $\left.64.680^{\circ} \mathrm{N} 170.412^{\circ} \mathrm{E}\right), 18$.VIII.1966, Gorodkov (3 $\sigma^{7} \sigma^{7}, 3$

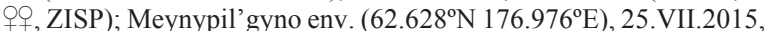
P.S. Tomkovich (1 $\left.\sigma^{T}, \mathrm{ZMUM}\right)$; Ugol'nyy env. $\left(64.733^{\circ} \mathrm{N}\right.$ $177.733^{\circ} \mathrm{E}$ ), 12-15.VIII.1966, Gorodkov (4 ऽ $\sigma^{7}, 2$ 오, ZISP); Ureliki $\left(64.400^{\circ} \mathrm{N} 173.214^{\circ} \mathrm{W}\right), 6$.VIII.1963, Gorodkov $\left(8 \sigma^{\top} \sigma^{\top}, 6\right.$ ㅇ, ZISP); Ivanovo Oblast: Malinki $\left(56.995^{\circ} \mathrm{N} 41.154^{\circ} \mathrm{E}\right)$, 2.IX.1969, Lobanov ( $\sigma^{7}, 1$, ZISP); Kamchatka Krai: Kozyrevsk $\left(56.048^{\circ} \mathrm{N} 159.870^{\circ} \mathrm{E}\right), 7 . I X .1969$, Gorodkov (6 $\sigma^{7} \sigma^{\top}, 4$ 우, ZISP); Zhupanovo $\left(54.082^{\circ} \mathrm{N} 159.974^{\circ} \mathrm{E}\right)$, 6.IX.1969, Gorodkov (4 $\sigma^{7} \sigma^{7}$, 5 우, ZISP); Commander Islands, Nikol'skoe $\left(55.196^{\circ} \mathrm{N} 165.998^{\circ} \mathrm{E}\right)$, 4-20.IX.1959, Gorodkov (12 $\sigma^{7} \sigma^{\top}, 7$ 우, ZISP); PetropavlovskKamchatskiy $\left(53.013^{\circ} \mathrm{N} 158.657^{\circ} \mathrm{E}\right)$, 5.IX.1969, Gorodkov $\left(5 \bigcirc^{7} \sigma^{7}\right.$, 1 ․ ZISP); Valley of Geysers (ca. $\left.54.43^{\circ} \mathrm{N} 160.15^{\circ} \mathrm{E}\right), 12$.VIII.1985, V. Zlobin (3 $\sigma^{7} \sigma^{7}, 2$ $9+$, ZISP); Karelia: Poyakonda $\left(66.589^{\circ} \mathrm{N}\right.$ $32.821^{\circ} \mathrm{E}$ ), 8.VII.2010, A.L. Ozerov (1 $\sigma^{7}$, ZMUM); Primorsky env. $\left(66.549^{\circ} \mathrm{N} 33.133^{\circ} \mathrm{E}, 66.552^{\circ} \mathrm{N} 33.100^{\circ} \mathrm{E}, 66.545^{\circ} \mathrm{N} 33.103^{\circ} \mathrm{E}\right)$, 30.VI., 3 and 7.VII.2010, A.L. Ozerov (2 $\sigma^{\top}, 2$, 2 , ZMUM); Pudozh $\left(66.804^{\circ} \mathrm{N} 36.565^{\circ} \mathrm{E}\right), 17$ and 19.VII.1982, Gorodkov (2 우, ZISP); Segezha $\left(63.745^{\circ} \mathrm{N} 34.317^{\circ} \mathrm{E}\right), 29 . \mathrm{VII} .1996$, Gorodkov (1 9 , ZISP); Sumskoy Posad $\left(64.238^{\circ} \mathrm{N} 35.408^{\circ} \mathrm{E}\right), 2$ 27.VII.1996, Gorodkov ( $1 \sigma^{7}$, ZISP); Khabarovsk Krai: Bulgino env. $\left(59.371^{\circ} \mathrm{N}\right.$

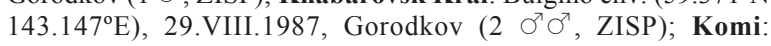
Shchel'yabozh $\left(66.29^{\circ} \mathrm{N} 56.45^{\circ} \mathrm{E}\right), 13$ and 15 .VIII.1978, Gorodkov (2 $\sigma^{\top} \sigma^{\top}$, ZISP); Sivaya Maska Station $\left(66.675^{\circ} \mathrm{N} 62.569^{\circ} \mathrm{E}\right), 12$ and 18.VII.1961, Gorodkov ( $1 \sigma^{\top}, 1$, ZISP); same place, but $5 \mathrm{~km}$ NW, 16.VII.1961, Gorodkov (1 ऽ, ZISP); Syktyvkar $\left(61.669^{\circ} \mathrm{N}\right.$ $\left.50.822^{\circ} \mathrm{E}\right), 29-30$.VII.1976 and 24.VII.1982, Gorodkov (4 $\sigma^{\top} \sigma^{\top}, 2$ of, ZISP); Ust-Tsilma $\left(65.440^{\circ} \mathrm{N} 52.153^{\circ} \mathrm{E}\right)$, 6.VIII.1978, Gorodkov (1 フ, ZISP); Ust'-Usa $\left(65.980^{\circ} \mathrm{N} 56.911^{\circ} \mathrm{E}\right), 13$. VIII.1978, Gorodkov (2 $\sigma^{\top} \sigma^{7}, 1$ \% , ZISP); Vorkuta $\left(67.501^{\circ} \mathrm{N} 64.039^{\circ} \mathrm{E}\right)$, 23.VII.1961, Gorodkov (4 $\bigcirc^{7} \sigma^{7}, 2$ OO. ZISP); same place, 1925.VII.2010, N. Vikhrev ( 8 ○ $^{7}, 3$ OO , ZMUM); Yaksha $\left(61.823^{\circ} \mathrm{N}\right.$ $\left.56.821^{\circ} \mathrm{E}\right), 12$.VIII.1989, Gorodkov ( 1 , ZISP); Krasnoyarsk Krai: Agapa $\left(71.421^{\circ} \mathrm{N} 89.251^{\circ} \mathrm{E}\right)$, River Pyasina, 15-21.VII.1967, Gorodkov $\left(5 \sigma^{\top} \sigma^{\top}, 3\right.$ 우, ZISP); Baykit $\left(61.68^{\circ} \mathrm{N} 96.38^{\circ} \mathrm{E}\right), 23$ and 26.VIII.1972, Gorodkov ( $4 \sigma^{7} \sigma^{7}, 4$ 우, ZISP); Dikson $\left(73.508^{\circ} \mathrm{N}\right.$ $\left.80.529^{\circ} \mathrm{E}\right)$, 8.VIII.1967, Gorodkov (1 $\sigma^{7}, 1$ ㅇ, ZISP); Dudinka $\left(69.404^{\circ} \mathrm{N} 86.182^{\circ} \mathrm{E}\right), 2$.VII.1967, Gorodkov (3 $\sigma^{7} \sigma^{7}$, ZISP); Igarka $\left(67.457^{\circ} \mathrm{N} 86.598^{\circ} \mathrm{E}\right)$, River Yenisei, 30.VI. and 1.VII.1967, Gorodkov (4 フ $\sigma^{7}, 3$ 우, ZISP); Lake Glubokoe, $60 \mathrm{~km}$ E of Noril'sk (ca.

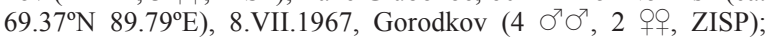


Noril'sk env., Medvezhiy (69.284 $\left.88.148^{\circ} \mathrm{E}\right)$, 6.VII.1967, Gorodkov ( $3 \sigma^{7} \sigma^{7}, 1$ ㅇ, ZISP); Noril'sk env., Talnakh $\left(69.500^{\circ} \mathrm{N} 88.447^{\circ} \mathrm{E}\right)$, 22.VIII.1973, Gorodkov (4 $\sigma^{7} \sigma^{\top}, 3$ 우, ZISP); Noril'sk env.

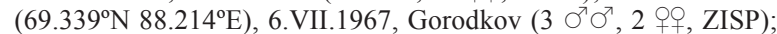
River Nizhnyaya Agapa, $40 \mathrm{~km}$ below the source $\left(\sim 70.097^{\circ} \mathrm{N}\right.$ $\left.86.688^{\circ} \mathrm{E}\right), 12-14$.VII.1973, V. Zherikhin \& I. Sukacheva $\left(2 \sigma^{7} \sigma^{7}\right.$, ZMUM); Kursk Oblast: Streletskaya Steppe $\left(51.579^{\circ} \mathrm{N} 36.087^{\circ} \mathrm{E}\right)$, 11-13.V.2008, K. Tomkovich (1 $\sigma^{7}$, ZMUM); Leningrad Oblast: Rakovichi $\left(58.650^{\circ} \mathrm{N} 29.836^{\circ} \mathrm{E}\right)$, 1.IX.1897, G. Pleske $\left(2 \sigma^{\top} \sigma^{\top}\right.$, ZISP); Magadan Oblast: Chaybukha $\left(61.801^{\circ} \mathrm{N} 160.413^{\circ} \mathrm{E}\right), 1$ and 2.IX.1987, Gorodkov (5 $\sigma^{7} \sigma^{\top}, 1 \sigma^{7}$, ZISP); Evensk $\left(61.916^{\circ} \mathrm{N}\right.$ $\left.159.234^{\circ} \mathrm{E}\right), 9 . I X .1987$, Gorodkov (1 ○', ZISP); Gizhiga $\left(61.948^{\circ} \mathrm{N}\right.$

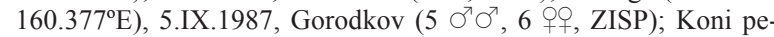
ninsula, Cape Ploskiy $\left(59.161^{\circ} \mathrm{N} 151.643^{\circ} \mathrm{E}\right), 16$. VII.2015, 3.VII. and 27.VIII.2017, N. Tridrikh (3 $\sigma^{7} \sigma^{7}$, ZMUM); Sokol $\left(59.919^{\circ} \mathrm{N}\right.$ $\left.150.752^{\circ} \mathrm{E}\right), 25$. VIII.1966, Gorodkov (1 $\sigma^{7}$, ZISP); Moscow and Moscow Oblast: $20 \mathrm{~km}$ WSW of Volokolamsk $\left(55.983^{\circ} \mathrm{N}\right.$ $\left.35.616^{\circ} \mathrm{E}\right)$, 11.VII.1999, A.L. Ozerov (1 9 , ZMUM); Burtsevo $\left(55.937^{\circ} \mathrm{N} 37.388^{\circ} \mathrm{E}\right)$, 13.V.2010, A.L. Ozerov (1 o', ZMUM); Dmitrov env. $\left(56.316^{\circ} \mathrm{N} 37.725^{\circ} \mathrm{E}\right)$, 9-23.IX.2006, 9, 18-19.V., 21.VI.2007, 8 and 18.V.2010, N. Vikhrev (8 $\sigma^{\top} \sigma^{7}, 5$ 우, ZMUM); Ivanovskoe $\left(55.933^{\circ} \mathrm{N} 35.623^{\circ} \mathrm{E}\right), 19 . \mathrm{V} .2006$, A.L. Ozerov (1 $\sigma^{7}$, ZMUM); Izmaylovo $\left(55.786^{\circ} \mathrm{N} 37.835^{\circ} \mathrm{E}\right), 12 . \mathrm{V} .1983$, A.L. Ozerov (1 ○, ZMUM); Molzhaninovka $\left(55.936^{\circ} \mathrm{N} 37.385^{\circ} \mathrm{E}\right)$, 13.V.2010, A.L. Ozerov (1 $\sigma^{7}$, ZMUM); Ozhigovo $\left(55.455^{\circ} \mathrm{N}\right.$ $36.882^{\circ}$ E), 29.IV.2008, 26.VII.2010, D. Gavryushin (1 9 , ZMUM); Shchukino $\left(55.798^{\circ} \mathrm{N} 37.478^{\circ} \mathrm{E}\right), 22 . I X .2006$, N. Vikhrev $(1$ q ZMUM); Smolevo $\left(55.626^{\circ} \mathrm{N} 38.964^{\circ} \mathrm{E}\right), 10-20 . X .2009$, K. Tomkovich ( 1 , ZMUM); Murmansk env. $\left(68.918^{\circ} \mathrm{N} 33.059^{\circ} \mathrm{E}\right), 9_{-}$ 13.VIII.2010, N. Vikhrev (1 + , ZMUM); same place, 3.VIII.1981, Gorodkov $\left(1 \sigma^{7}, 1\right.$ ․ ZISP); $8 \mathrm{~km} \mathrm{~N}$ of Revda $\left(68.003^{\circ} \mathrm{N} 34.570^{\circ} \mathrm{E}\right)$, 15.VIII.1981, Gorodkov (1 9 , ZISP); Aleksandrovsk [= Polyarny] $\left(69.198^{\circ} \mathrm{N} 33.456^{\circ} \mathrm{E}\right), 1 . \mathrm{V} ., 14$ and 18.VI.1921, Zhelokhovtsev (3 $\sigma^{7} \sigma^{7}$, ZMUM); Vudyavr Lake basin (ca. $\left.67.646^{\circ} \mathrm{N} 33.644^{\circ} \mathrm{E}\right), 18 . \mathrm{VI}$

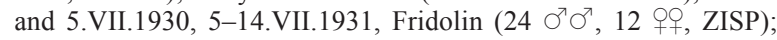
Dal'nie Zelentsy $\left(69.117^{\circ} \mathrm{N} 36.062^{\circ} \mathrm{E}\right)$, 6.VIII.1981, Gorodkov (1 $\sigma^{7}, 1$ q, ZISP); Khibiny Station $\left(67.673^{\circ} \mathrm{N} 33.212^{\circ} \mathrm{E}\right), 27$. VIII.1928, Cheburova (1 $\mathrm{O}, \mathrm{ZISP})$; Kirovsk env. $\left(67.608^{\circ} \mathrm{N} 33.661^{\circ} \mathrm{E}\right)$, 9.VII.1975, Zinov'ev (2 of, ZISP); Kovdor $\left(67.564^{\circ} \mathrm{N} 30.478^{\circ} \mathrm{E}\right)$, 21.VIII.1995, Gorodkov (3 $\sigma^{7} \sigma^{\top}, 3$ 우, ZISP); Laplandskiy Nature Reserve $\left(67.926^{\circ} \mathrm{N} 32.052^{\circ} \mathrm{E}\right), 19 . \mathrm{VIII} .1976, \mathrm{~V}$. Sychevskaya (1 $q$, ZMUM); Lovozero $\left(68.005^{\circ} \mathrm{N} 35.017^{\circ} \mathrm{E}\right), 14$.VIII.1981, Gorodkov (1 ㅇ, ZISP); Monchegorsk (67.937 $\left.{ }^{\circ} \mathrm{N} 32.891^{\circ} \mathrm{E}\right), 11-18 . \mathrm{VII} .2009$, M. Kozlov (1 +, ZMUM); Murmansk env. $\left(68.978^{\circ} \mathrm{N} 33.115^{\circ} \mathrm{E}\right.$, $\left.68.973^{\circ} \mathrm{N} 33.137^{\circ} \mathrm{E}, 68.978^{\circ} \mathrm{N} 33.117^{\circ} \mathrm{E}, 68.979^{\circ} \mathrm{N} 33.151^{\circ} \mathrm{E}\right), 18-$ 21.VII.2011, A. Ozerov, D. Gavryushin (5 $\sigma^{\top} \sigma^{7}, 1$ \%, ZMUM); Tumannyy $\left(68.883^{\circ} \mathrm{N} 35.693^{\circ} \mathrm{E}\right), 4$.VIII.1981, Gorodkov (1 + , ZISP); Novgorod Oblast: Novgorod env. $\left(58.486^{\circ} \mathrm{N} 31.284^{\circ} \mathrm{E}\right)$, 1.IX.1978,

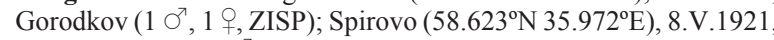
Zhelokhovtsev (1 $\sigma^{7}$, ZMUM); Novosibirsk Oblast: Novosibirsk $\left(54.842^{\circ} \mathrm{N} 83.114^{\circ} \mathrm{E}\right), 9-10 . I X .2009$, O. Kosterin (3 $\sigma^{7} \sigma^{7}$, ZMUM); Pskov Oblast: Aparino $\left(56.958^{\circ} \mathrm{N} 29.966^{\circ} \mathrm{E}\right), 16 . \mathrm{V} .1915, \mathrm{~N}$. Kuznetsov (1 $\sigma^{r}$, ZISP); Rostov Oblast: Kamensk-Shakhtinsky $\left(48.344^{\circ} \mathrm{N} 40.257^{\circ} \mathrm{E}\right), 24 . V .2011$, D. Gavryushin (1 $\sigma^{7}$, ZMUM); Sakhalin Oblast: Iturup I., Cape Burevestnik $\left(44.921^{\circ} \mathrm{N} 147.65^{\circ} \mathrm{E}\right)$ 28.VI.1954, Violovich (1 $0^{7}, 1$ \% , ZISP); Iturup I., Rybaki $\left(45.209^{\circ} \mathrm{N}\right.$ 147.851 ${ }^{\circ}$ E), 22.VI.1968, V. Richter (1 $\sigma^{7}$, ZISP); Paramushir I., Severo-Kuril'sk $\left(50.679^{\circ} \mathrm{N} 156.132^{\circ} \mathrm{E}\right), 8$ and 14.IX.1968, Gorodkov (2 우, ZISP); Paramushir I., Severo-Kuril'sk $\left(50.679^{\circ} \mathrm{N}\right.$ $\left.156.132^{\circ} \mathrm{E}\right), 7-14 . I X .1968$, Gorodkov (6 $\sigma^{7} \sigma^{7}, 4$ 우, ZISP); Tver' Oblast: Udomlya env. $\left(57.881^{\circ} \mathrm{N} 35.008^{\circ} \mathrm{E}\right), 4 . X .2003,8-$ 31.X.2009, A. Korobkov (1 $\sigma^{7}, 2$ 우, ZISP); Tyumen' Oblast: $\left(63.766^{\circ} \mathrm{N} 59.716^{\circ} \mathrm{E}, 63.818^{\circ} \mathrm{N} 59.562^{\circ} \mathrm{E}\right), 1-8$.VII.2010, K. Tomkovich (3 $\sigma^{7} \mathrm{O}^{7}, 1$ \% , ZMUM); $130 \mathrm{~km} \mathrm{SE}$ of Nadym (ca. $64.718^{\circ} \mathrm{N}$ $\left.74.532^{\circ} \mathrm{E}\right), 2$. VIII.1977, Gorodkov (2 90 , ZISP); $75 \mathrm{~km}$ WSW of Samburg (ca. $67.035^{\circ} \mathrm{N} 76.541^{\circ} \mathrm{E}$ ), 7-9.VIII.1976, Gorodkov (5 $0^{7} \sigma^{7}, 3$ o, ZISP); $83 \mathrm{~km}$ WNW of Tazovskiy (ca. $68.049^{\circ} \mathrm{N}$ 77.702 ${ }^{\circ} \mathrm{E}$ ), 29.VII.1977, Gorodkov (7 $\sigma^{\top} \sigma^{\top}, 3$ oO, ZISP);Voykar River basin (ca. $65.720^{\circ} \mathrm{N} 64.324^{\circ} \mathrm{E}$ ), 8-18.VIII.1925, Fridolin (5 Oフ $^{7}, 5$ 우, ZISP); Bely Nos $\left(69.618^{\circ} \mathrm{N} 60.223^{\circ} \mathrm{E}\right), 4-18$. VII. 1957 (3 $\sigma^{7} \sigma^{7}, 1$ \%, ZMUM); Berezovo (63.937 $\left.{ }^{\circ} \mathrm{N} 65.041^{\circ} \mathrm{E}\right), 26 . \mathrm{VIII} .1976$, Gorodkov (3 O $^{7}$, 2 우, ZISP); Gaz-sale $\left(67.364^{\circ} \mathrm{N} 78.998^{\circ} \mathrm{E}\right), 18$ km SE of Tazovskiy, 31.VII.1977, Gorodkov (3 $\sigma^{7} \sigma^{7}, 3$ + + , ZISP);
Khanty-Mansiysk $\left(61.000^{\circ} \mathrm{N} 69.032^{\circ} \mathrm{E}\right), 27-28$.VIII.1976, Gorod-

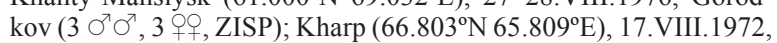

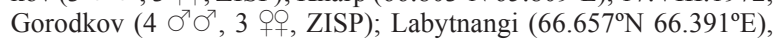
30.VI., 10-28.VII., 16.VIII.1973, R. Kamenskaya, V. Sychevskaya

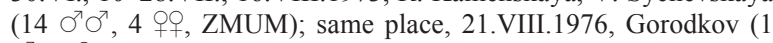
${ }^{7}, 1$, ZISP); Muzhi $\left(65.397^{\circ} \mathrm{N} 64.700^{\circ} \mathrm{E}\right)$, 22.VIII.1976, Gorodkov $\left(6 \bigcirc^{7} \sigma^{7}, 1\right.$, ZISP); Cape Kamennyy $\left(72.066^{\circ} \mathrm{N} 77.434^{\circ} \mathrm{E}\right)$,

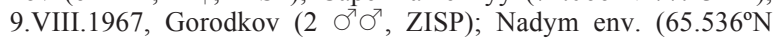
$\left.72.522^{\circ} \mathrm{E}\right), 6,12$ and 15.VIII.1976, Gorodkov (3 $\sigma^{\top} \sigma^{\top}, 2$ 오, ZISP); Novyy Urengoy $\left(66.087^{\circ} \mathrm{N} 76.513^{\circ} \mathrm{E}\right), 30 . \mathrm{VII} .1982$, Gorodkov (5 $\sigma^{7} \sigma^{7}, 5$ 90 , ZISP); Nyda $\left(66.628^{\circ} \mathrm{N} 72.922^{\circ} \mathrm{E}\right), 3-5 . V I I I .1977$, Gorodkov (7 $\sigma^{7} \sigma^{7}, 2$ 90 , ZISP); River Shchuch'ya $\left(60.564^{\circ} \mathrm{N}\right.$ $\left.68.797^{\circ} \mathrm{E}\right), 11 . \mathrm{VII} .1984$, P. Basikhin (1 $\sigma^{7}$, 1 \%, ZMUM); Salekhard $\left(66.53^{\circ} \mathrm{N} 66.613^{\circ} \mathrm{E}\right), 3-5$.VIII.1976, Gorodkov (8 ${ }^{7} \mathrm{O}^{7}, 3$ 우, ZISP); Salekhard $\left(66.53^{\circ} \mathrm{N} 66.613^{\circ} \mathrm{E}\right)$, VI.1951 (1 O', ZISP); Sob' env. $\left(67.07^{\circ} \mathrm{N} 65.46^{\circ} \mathrm{E}\right), 26-31 . V I I .2011, \mathrm{~K}$. Tomkovich (1 ㅇ, ZMUM); Tarko-Sale $\left(64.914^{\circ} \mathrm{N} 77.766^{\circ} \mathrm{E}\right), 1$ and 2.IX.1982, Gorodkov (4 $\sigma^{7} \sigma^{7}, 2$ 우, ZISP); Tobol'sk $\left(58.206^{\circ} \mathrm{N} 68.264^{\circ} \mathrm{E}\right), 2 . I X .1925$, Fridolin ( $2 \sigma^{7} \sigma^{7}, 1$ \%, ZISP); Tyumen' $\left(57.150^{\circ} \mathrm{N} 65.535^{\circ} \mathrm{E}\right)$, 4.IX.1976, Gorodkov ( $4 \sigma^{7} \sigma^{7}, 1$, ZISP); Vaygach, Cape Lyamchin $\left(69.870^{\circ} \mathrm{N} 59.136^{\circ} \mathrm{E}\right), 13$.VIII.1957 (1 ${ }^{7}, 1$ O , ZMUM); Yar-Sale $\left(66.863^{\circ} \mathrm{N} 70.83^{\circ} \mathrm{E}\right), 28$. VII.1986, Veselkin (1 + , ZMUM); Vologda Oblast: Belozersk env. $\left(60.029^{\circ} \mathrm{N} 37.791^{\circ} \mathrm{E}\right), 13$.VI.1904, Boro-

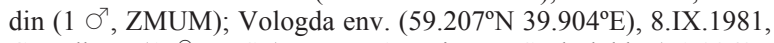
Gorodkov (1 9 , ZISP); Yakutia: airport Saskylakh $\left(71.934^{\circ} \mathrm{N}\right.$ $\left.114.083^{\circ} \mathrm{E}\right), 24$. VII.1988, Gorodkov (1 9 , ZISP); Aykhal $\left(65.944^{\circ} \mathrm{N}\right.$ $\left.111.495^{\circ} \mathrm{E}\right), 19$. VIII.1988, Gorodkov (3 우, ZISP); Chekurovka $\left(71.046^{\circ} \mathrm{N} 127.525^{\circ} \mathrm{E}\right), 19$ and 24.VII.1957, Gorodkov (6 $\sigma^{7} \sigma^{7}, 4$ 우, ZISP); Chersky $\left(68.75^{\circ} \mathrm{N} 161.332^{\circ} \mathrm{E}\right), 6$.VII.1971, Gorodkov (1 $\sigma^{7}$, ZISP); Chokurdakh $\left(70.618^{\circ} \mathrm{N} 147.895^{\circ} \mathrm{E}\right), 20-21 . V I I .1971$, Gorodkov (2 O $^{7}, 3$ 오, ZISP); Deputatskiy $\left(69.319^{\circ} \mathrm{N} 139.966^{\circ} \mathrm{E}\right)$, 6-7.VIII.1974, Gorodkov (6 O' $\sigma^{7}, 4$ 우, ZISP); Ebelyakh, bank of the River Anabara $\left(70.884^{\circ} \mathrm{N} 113.57^{\circ} \mathrm{E}\right), 14$.VIII.1988, Gorodkov (1 $\sigma^{\gamma}$, ZISP); env. of Ystannakh-Khocho Vill. (ca. $72.58^{\circ} \mathrm{N}$, $\left.121.42^{\circ} \mathrm{E}\right)$, 20.VIII.2010, A. Yadrenkin (1 $\sigma^{7}$, ZMUM); Kular $\left(70.573^{\circ} \mathrm{N} 134.271^{\circ} \mathrm{E}\right), 2$.VIII.1974, Gorodkov (1 9 , ZISP); same place, 24-25.VII.2000, Potapova (4 $\sigma^{\top} \sigma^{\top}, 2$ 우, ZISP); Kular $\left(70.573^{\circ} \mathrm{N} 134.271^{\circ} \mathrm{E}\right), 3-4$. VIII.1974, Gorodkov (5 $\sigma^{7} \sigma^{7}, 3$ 우, ZISP); Mirny $\left(62.540^{\circ} \mathrm{N} 113.962^{\circ} \mathrm{E}\right), 16 . \mathrm{IX} .1987$, Gorodkov (1 o', ZISP); River Yana, Stolby env. (67.531 $\left.{ }^{\circ} \mathrm{N} 134.087^{\circ} \mathrm{E}\right), 27$. VII. 2008, A. Ovchinnikov (1 $\sigma^{7}$, ZISP); Tiksi env. $\left(71.635^{\circ} \mathrm{N} 128.857^{\circ} \mathrm{E}\right)$, 9.VII. and 10-13.VIII.1957, Gorodkov (5 $\sigma^{7} \sigma^{7}, 6$ 우, ZISP); Tostuya $\left(73.208^{\circ} \mathrm{N} 113.606^{\circ} \mathrm{E}\right), 45 \mathrm{~km} \mathrm{~S}$ of Uryung-Khaya, 26.VII.1988, Gorodkov (1 $\sigma^{7}, 1$ ๆ, ZISP); Udachnyy $\left(66.405^{\circ} \mathrm{N} 112.299^{\circ} \mathrm{E}\right)$, 20.VII.1988, Gorodkov (1 $\sigma^{7}, 1$, ZISP). See also Ozerov \& Krivosheina [2014: 217] and Ozerov \& Barkalov [2014: 563].

DESCRIPTION. Male. Female (Fig. 3). Body-length 4.2-7.2 $\mathrm{mm}$.

Head. Frontal vitta reddish-yellow, matt; frontoorbital plate blackish, greyish dusted. Face, parafacial and gena yellow, with delicate golden reflection. Postcranium black in upper third or quarter and yellow in lower part. 3 orbital and 3-4 frontal setae present. Scapus and pedicel reddish-yellow. Postpedicel from reddish-yellow to blackish, but basally outside always reddish-yellow, approximately twice as long as wide. Arista bare. Palpus yellow.

Thorax black, greyish dusted. Acrostichals not differentiated from the other hairs on scutum or setulose in two irregular rows, but prescutellar pair usually stronger than the other acrostichals, dorsocentrals $2+3$, intra-alars $1+2$, supra-alars $1+2$, postpronotals 2 . Anepimeron bare. Scutellum black, greyish dusted, with a pair of strong basal scutellar and a pair of strong apical scutellar setae.

Legs yellow, but as a rule fore femur blackish dorsoventrally (Fig. 3). Fore femur covered with hairs, but without conspicuous setae. Fore tibia with 2-3 dorsal, 
0-1 posterior, 1 preapical dorsal, and 1 posterior apical setae. Mid femur with 3-5 anterodorsal setae in apical third in male and usually with a row of anterodorsal setae in female, also with 1 preapical posterior and 1 preapical posterodorsal setae in both sexes. Mid tibia with 3 posterodorsal, 2-3 anterodorsal, 1 anteroventral, 1 posteroventral setae and a ring of apicals. Hind femur with a row of anterodorsal setae in apical half in male and on all length in female. Hind tibia with 3 anterodorsal, 3 posterodorsal, 1 anteroventral, 1 preapical dorsal, 1 preapical anterodorsal, 1 apical anteroventral setae.

Wing tinged with brownish; crossveins r-m and dmcu darkened (Fig. 3).

Abdomen from yellow to dark brownish, delicately greyish dusted, covered with not long hairs, in female tergites with black marginal setulae. Male sternite 4 approximately 1.5 times as long as wide (Fig. 22); sternite 5 with a pair of small apical median processes, covered with spinules (Fig. 67). Epandrium, cercal plate and surstyli as in Figs 68, 69.

DISTRIBUTION. Throughout Russia. - Europe (widespread); Japan, Mongolia [Šifner, 2008: 164]; North America; Haiti [Vockeroth, 2010: 1269]; common in forest zone.

\section{Scathophaga incola (Becker, 1900)}

Figs 9, 23, 70-72.

incola Becker, 1900: 54 (Scatophaga). Type-locality: "Kantaika und der Insel Nikander" [Khantayka and Nikandorskie Islands NE of Dudinka] (Russia: Krasnoyarsk Krai).

fascifrons Ringdahl, 1936: 174 (Scopeuma). Type-locality: "Moore bei Abisco in Lappland angetroffen" (Sweden).

REMARK. This species was recorded in Russia for subarctic and taiga zones in European part [Gorodkov,1986: 31 (without specifying the place of collecting); Engelmark, 1999: 158, 159; Polevoi, 1997: 309], Siberia [Becker, 1900: 54; Ozerov, Barkalov, 2014: 563], Yakutia [Veselkin, 1985: 75; Bagachanova et al. 2016: 782] and Far East [Hendel, 1930: 2; Ozerov, Krivosheina, 2014: 217].

MATERIAL EXAMINED. Altai: "Rodonovy istochnik", 2412 m, Lake Muzdy-Bulak (49.46 $\left.\mathrm{N} 88.05^{\circ} \mathrm{E}\right)$, 5.VII.2008, A. Barkalov (2 $\sigma^{7} \sigma^{7}$, ISEA); Kosh-Agach, $2400 \mathrm{~m}$, Lake Muzdy-Bulak env. $\left(49.26^{\circ} \mathrm{N}, 87.65^{\circ} \mathrm{E}\right), 8-10 . \mathrm{VII} .2008$, A. Barkalov $\left(6\right.$ ○ $^{\top} \sigma^{7}, 4$ 우, ISEA and ZMUM); Kosh-Agach, plateau Ukok, 2450 m, Lake Muzdy-Bulak $\left(49.3^{\circ} \mathrm{N} 87.65^{\circ} \mathrm{E}\right), 8$.VII.2008, A. Barkalov (2 $0^{7} 0^{7}$, 5 우, ISEA and ZMUM); Topuchaya Vill. $\left(51.126^{\circ} \mathrm{N} 85.586^{\circ} \mathrm{E}\right)$, 1825 m, 3-4.VII.2014, I. Shamshev (1 ○', 2 우, ZISP); Arkhangelsk Oblast: Karpogory $\left(63.999^{\circ} \mathrm{N} 44.453^{\circ} \mathrm{E}\right)$, 10.VII.1996, Gorodkov (1 9 , ZISP); Buryatia: Barguzinsky Nature Reserve (ca. $54.35^{\circ} \mathrm{N}$ $\left.109.51^{\circ} \mathrm{E}\right), 4$ and 16.VII.1965, Negrobov ( $1 \mathrm{O}^{2}, 1$, , ZISP); Mondy env. $\left(51.675^{\circ} \mathrm{N} 100.992^{\circ} \mathrm{E}\right), 28$.VII.1965, Gorodkov (2 $0^{7} \mathrm{O}^{7}$, ZISP); Chukotka: $20 \mathrm{~km} \mathrm{SSO}$ of Iul'tin $\left(67.724^{\circ} \mathrm{N} 178.515^{\circ} \mathrm{W}\right)$, 22.VII.1963, Gorodkov ( 1 + ZISP); Chaunskaya Bay, the mouth of the River Ichun' $\left(68.854^{\circ} \mathrm{N} 170.547^{\circ} \mathrm{E}\right)$, 3.VII.1963, Semenov (5 $\mathrm{O}^{7} \mathrm{O}^{7}, 4$ 우, ZISP); Markovo (ca. $\left.64.680^{\circ} \mathrm{N} 170.412^{\circ} \mathrm{E}\right), 18 . \mathrm{VIII} .1966$ Gorodkov (2 $\sigma^{7} \sigma^{7}$, ZISP); Meynypil'gyno env. $\left(62.628^{\circ} \mathrm{N}\right.$ $\left.176.976^{\circ} \mathrm{E}\right)$, 14.VII.2015, P.S. Tomkovich (1 $\sigma^{7}$, ZMUM); Pevek $\left(69.702^{\circ} \mathrm{N} 170.298^{\circ} \mathrm{E}\right), 11$. VIII.1963, Gorodkov (1 $\sigma^{7}, 1$ \% , ZISP); Kamchatka Krai: Apuka env. $\left(60.443^{\circ} \mathrm{N} 169.602^{\circ} \mathrm{E}\right), 7 . V I I I .1959$, Gorodkov (1 $\bigcirc^{7}$, ZISP); Krapivnoe, $60 \mathrm{~km} \mathrm{E}$ of Esso $\left(55.927^{\circ} \mathrm{N}\right.$ $\left.158.704^{\circ} \mathrm{E}\right), 8$.VIII.1978, A. Zinov'ev (1 9 , ZISP); Karelia: Kartesh $\left(66.33^{\circ} \mathrm{N} 33.64^{\circ} \mathrm{E}\right), 22 . V I I .1975$, Gorodkov (1 $\bigcirc^{\top}$, ZISP); Khabarovsk Krai: Bulgino env. $\left(59.371^{\circ} \mathrm{N} 143.147^{\circ} \mathrm{E}\right)$, 29.VIII.1987, Gorodkov (1 $\sigma^{7}, 2$ 우, ZISP); Komi: Blagoevo $\left(63.41^{\circ} \mathrm{N} 47.95^{\circ} \mathrm{E}\right), 12$ and 13.VII.1996, Gorodkov (2 우, ZISP); Krasnodar Krai: Teberdinskiy Nature Reserve, Dzhamagat Valley $\left(43.464^{\circ} \mathrm{N} 41.736^{\circ} \mathrm{E}\right), 7$. VII.1984, Ovchinnikov (1 $0^{7}$, ZISP); Krasnoyarsk Krai: Noril'sk env. $\left(69.339^{\circ} \mathrm{N} 88.214^{\circ} \mathrm{E}\right)$, 4.VII.1967, 21.VIII.1973, Gorodkov (2 $0^{7} \mathrm{O}^{7}$, ZISP); Zhdanikha $\left(72.171^{\circ} \mathrm{N}\right.$, $\left.102.868^{\circ} \mathrm{E}\right), 27-30$. VIII.1971, V. Zherikhin \& I. Sukacheva (1 $0^{7}$, ZMUM); Magadan Oblast: Chaybukha $\left(61.801^{\circ} \mathrm{N} 160.413^{\circ} \mathrm{E}\right)$, 1.IX.1987, Gorodkov (1 9 , ZISP); Moscow Oblast: Dmitrov env. $\left(56.316^{\circ} \mathrm{N} 37.725^{\circ} \mathrm{E}\right)$, 8.IX.2006, N. Vikhrev (1 +. ZMUM); Murmansk Oblast: River Tuloma (ca. $68.738^{\circ} \mathrm{N} 32.288^{\circ} \mathrm{E}$ ), $4-$ 6.VIII.1904, Soldatov ( $20^{7} \sigma^{7}$, ZISP); Primorsky Krai: Mt Oblachnaya $\left(43.695^{\circ} \mathrm{N} 134.201^{\circ} \mathrm{E}\right), 11$. VIII.1963, Nartshuk (1 $\sigma^{7}$, ZISP); Tuva: River Uyuk $\left(52.07^{\circ} \mathrm{N} 94.04^{\circ} \mathrm{E}\right), 800 \mathrm{~m}, 1-3$. VII.2017, N. Vikhrev (1 $\mathrm{O}^{\top}$, ZMUM); the upper reaches of the River MogunBuren' (ca. $\left.50.041^{\circ} \mathrm{N} 89.872^{\circ} \mathrm{E}\right), 25 . \mathrm{VII} .1964$, Nartshuk (1 $\sigma^{7}$, ZISP); Tyumen' Oblast: $\left(63.818^{\circ} \mathrm{N} 59.562^{\circ} \mathrm{E}\right), 6-8 . \mathrm{VII} .2010$, K. Tomkovich (1 $\sigma^{7}$, ZMUM); $55 \mathrm{~km} \mathrm{~W}$ of Samburg (ca. $67.035^{\circ} \mathrm{N}$ $\left.76.541^{\circ} \mathrm{E}\right), 12$.VIII.1976, Gorodkov (1 $\mathrm{O}^{7}$, ZISP); $75 \mathrm{~km}$ WSW of Samburg (ca. $\left.67.0357^{\circ} \mathrm{N} 76.5411^{\circ} \mathrm{E}\right), 9$. VIII.1976, Gorodkov (1 9 , ZISP); $85 \mathrm{~km} \mathrm{WSW}$ of Antipayuta (ca. $68.820^{\circ} \mathrm{N} 75.344^{\circ} \mathrm{E}$ ), 31.VII.1977, Gorodkov (1 9 , ZISP); $87 \mathrm{~km} \mathrm{NW}$ of Tazovskiy (ca. $67.522^{\circ} \mathrm{N} 77.807^{\circ} \mathrm{E}$ ), floodplain of the River Khariyanog, 29.VII.1977, Gorodkov (1 9 , ZISP); Labytnangi env. $\left(66.661^{\circ} \mathrm{N}\right.$ 66.394 ${ }^{\circ}$ E), 20.VIII.1976, Gorodkov (1 $\mathrm{O}^{\top}$, ZISP); Nyda $\left(66.628^{\circ} \mathrm{N}\right.$ $\left.72.922^{\circ} \mathrm{E}\right), 5 . \mathrm{VIII} .1977$, Gorodkov (1 9 , ZISP); Saranpaul' $\left(64.257^{\circ} \mathrm{N}\right.$ 60.917 $\left.{ }^{\circ} \mathrm{E}\right)$, 30.VII.1990, Malozemov (1 $\sigma^{7}, 1$ ㅇ, ZISP); Tobol'sk env. (58.206 $\left.{ }^{\circ} \mathrm{N} 68.264^{\circ} \mathrm{E}\right)$, Lake Boyarskoe, 25.IX.1926, K.P. Samko (1 $0^{7}$, ZISP); Yakutia: Kular $\left(70.573^{\circ} \mathrm{N} 134.271^{\circ} \mathrm{E}\right), 3-4$.VIII.1974, Gorodkov (3 $\sigma^{7} \sigma^{7}, 8$ 우, ZISP); left bank of the River Yana opposite of Verkhoyansk $\left(67.55^{\circ} \mathrm{N} 133.359^{\circ} \mathrm{E}\right)$, 23.VII.1974, Nartshuk $\left(1 \mathrm{O}^{7}\right.$, ZISP); River Atirayyana near Zhigansk $\left(66.766^{\circ} \mathrm{N} 123.372^{\circ} \mathrm{E}\right)$, 12.VII.1875, Chekanovskiy ( 1 + , ZISP); Srednekolymsk $\left(67.46^{\circ} \mathrm{N}\right.$ $\left.153.71^{\circ} \mathrm{E}\right)$, 16.VIII.1974, Gorodkov (4 $\mathrm{O}^{7} \mathrm{O}^{7}$, ZISP); Tiksi env. $\left(71.635^{\circ} \mathrm{N} 128.857^{\circ} \mathrm{E}\right)$, 9.VII.-17.VIII.1957, Gorodkov (3 $0^{7} 0^{7}, 3$ o+o, ZISP); same place, 30.VII.1990, Kasparyan (1 + ZISP); Verkhoyansk $\left(67.548^{\circ} \mathrm{N} 133.396^{\circ} \mathrm{E}\right), 11$. VIII.1972, 22.VII.1974, Gorodkov ( $10^{7}, 1$, ZISP); Zabaikalskiy Krai: Lesnaya Station $\left(51.764^{\circ} \mathrm{N} 112.999^{\circ} \mathrm{E}\right), 1$.VII.2012, A. Medvedev (2 $0^{7} 0^{7}$ ZMUM). See also Ozerov \& Krivosheina [2014: 217] and Ozerov \& Barkalov [2014: 563].

DESCRIPTION. Male. Female. Body-length 6.5$10.6 \mathrm{~mm}$.

Head. Frontal vitta reddish-yellow, with delicate whitish reflection; fronto-orbital plate blackish, greyish dusted. Face, parafacial and gena reddish-yellow, with delicate whitish reflection. Postcranium black in upper third or quarter and reddish-yellow in lower part. 3 orbital and 4-5 frontal setae present. Antenna reddish-yellow. Postpedicel approximately twice as long as wide. Arista pubescent, the longest hairs approximately equal to $1 / 2$ width of postpedicel. Palpus yellow.

Thorax greyish dusted, black completely, only postpronotal lobe usually yellowish. Acrostichals setulose in two rows, but prescutellar pair stronger than the other acrostichals, dorsocentrals $2+3$, intra-alars $1+2$, supra-alars $1+2$, postpronotals 2 . Katepisternum posteriorly, besides yellow hairs and setulae, also with several black setulae. Anepimeron bare. Scutellum black, greyish dusted, with a pair of strong basal scutellar and a pair of strong apical scutellar setae.

Legs. Coxae from yellow to brown. Femora, tibiae, and tarsi yellowish, fore femur as a rule darkened posteriorly or posterodorsally. Fore femur covered with hairs, but without conspicuous setae. Fore tibia with $2-$ 

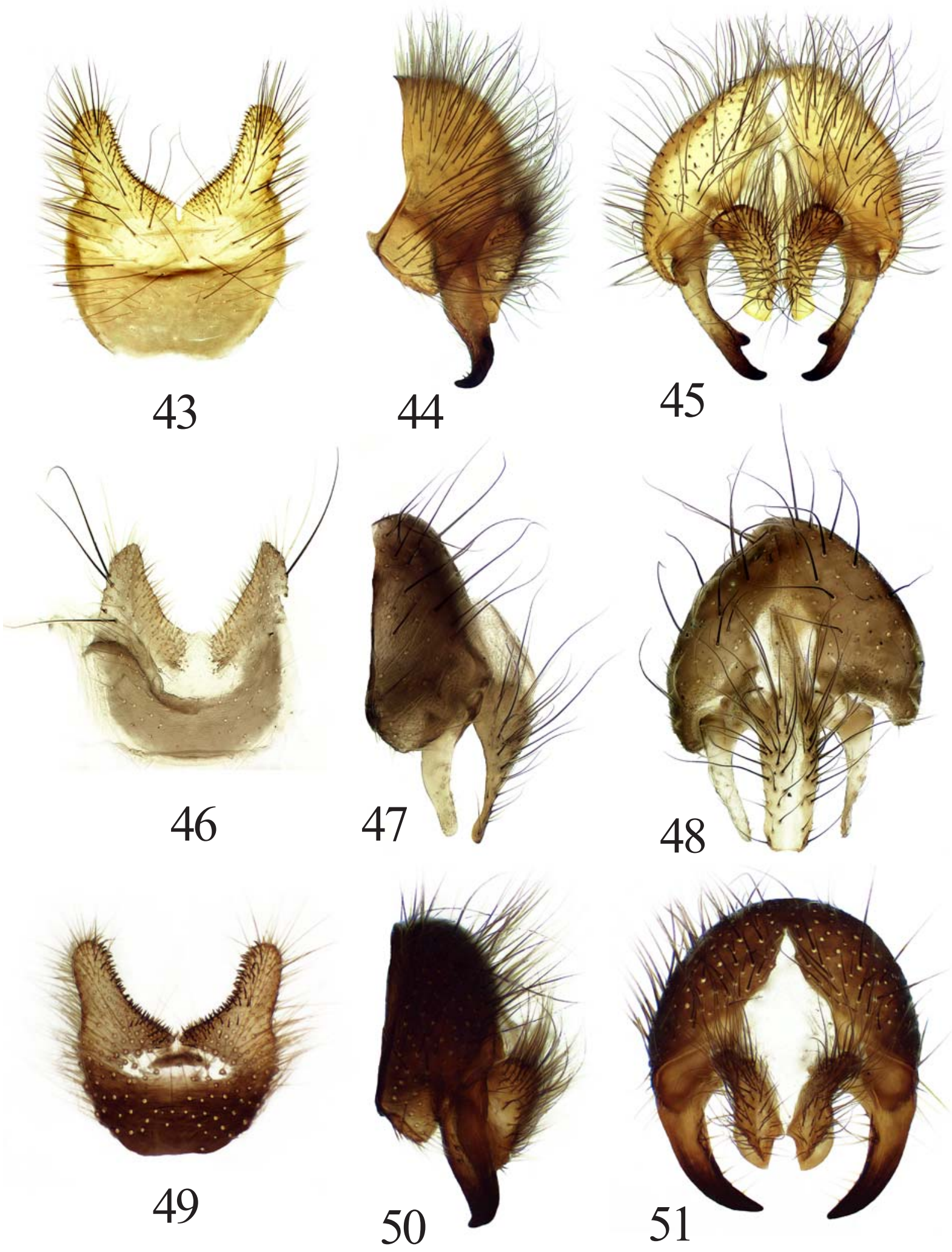

Figs 43-51. Scathophaga apicalis (Curtis) (43-45), S. buryatica Ozerov et Krivosheina (46-48) and S. calida (Haliday) (49-51): 43, 46, 49 - male sternite 5; 44, 47, 50 - epandrium, cercal plate and surstyli, lateral view; 45, 48, 51 - same, dorsal view. 46-48 - after Ozerov \& Krivosheina, 2019, figs 1, 3, 4.

Рис. 43-51. Scathophaga apicalis (Curtis) (43-45), S. buryatica Ozerov et Krivosheina (46-48) и S. calida (Haliday) (49-51): 43, 46, 49 - стернит 5 самца; 44, 47, 50 - эпандрий, церки и сурстили, сбоку; 45, 48, 51 - то же, сверху. 46-48 - по Ozerov \& Krivosheina, 2019, figs $1,3,4$. 


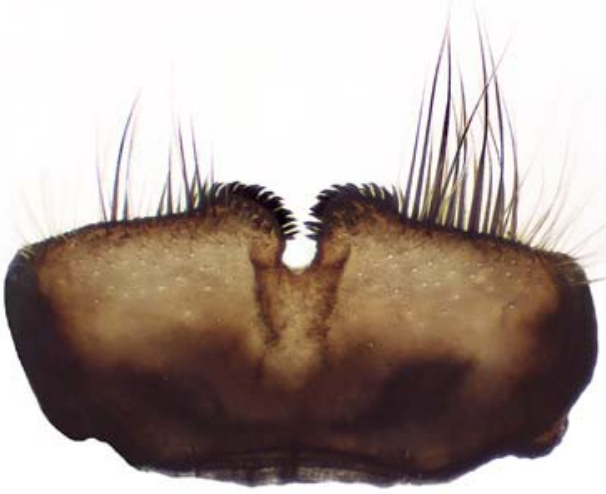

52

55

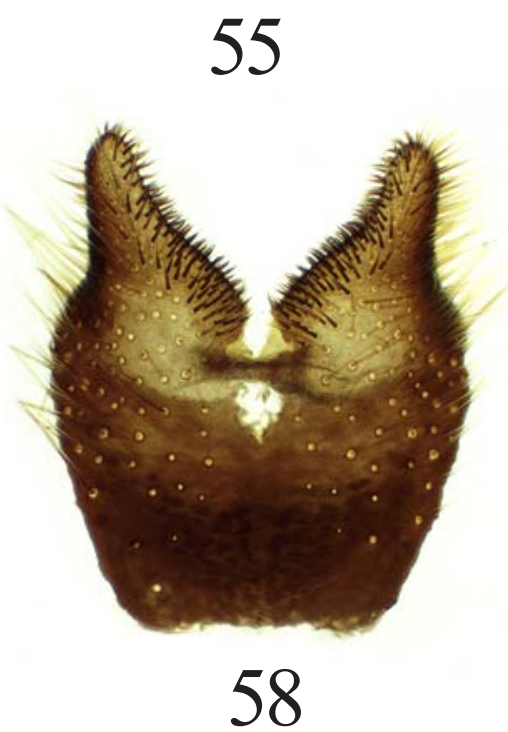

58

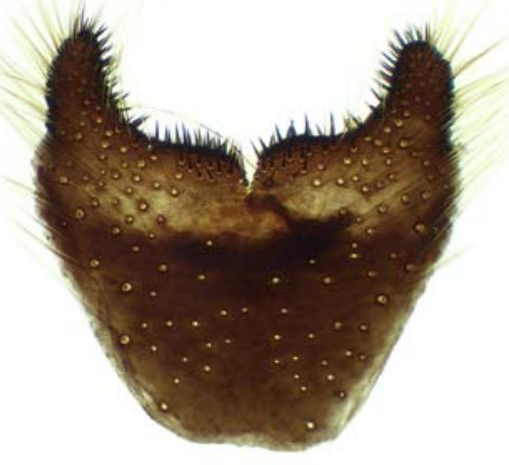

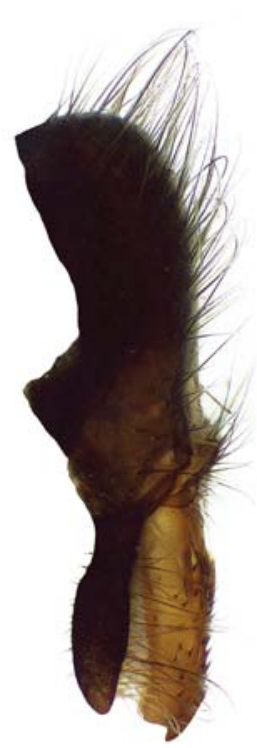

53
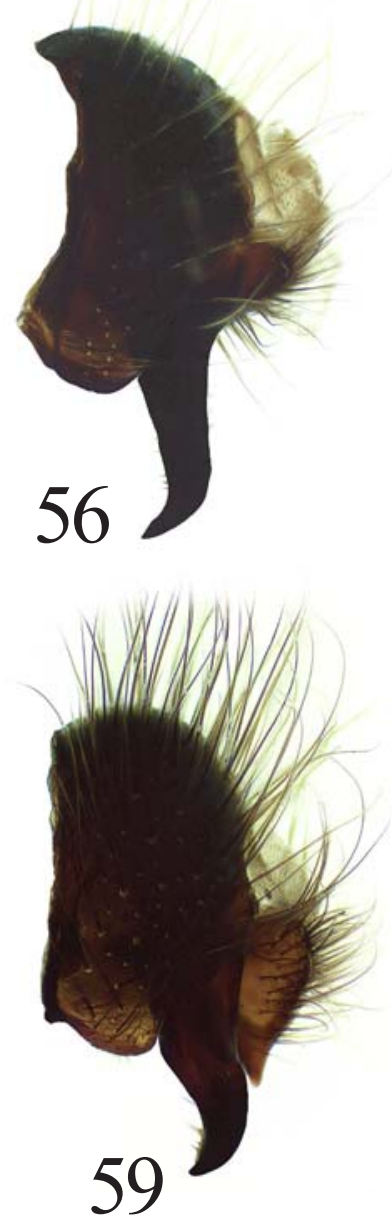
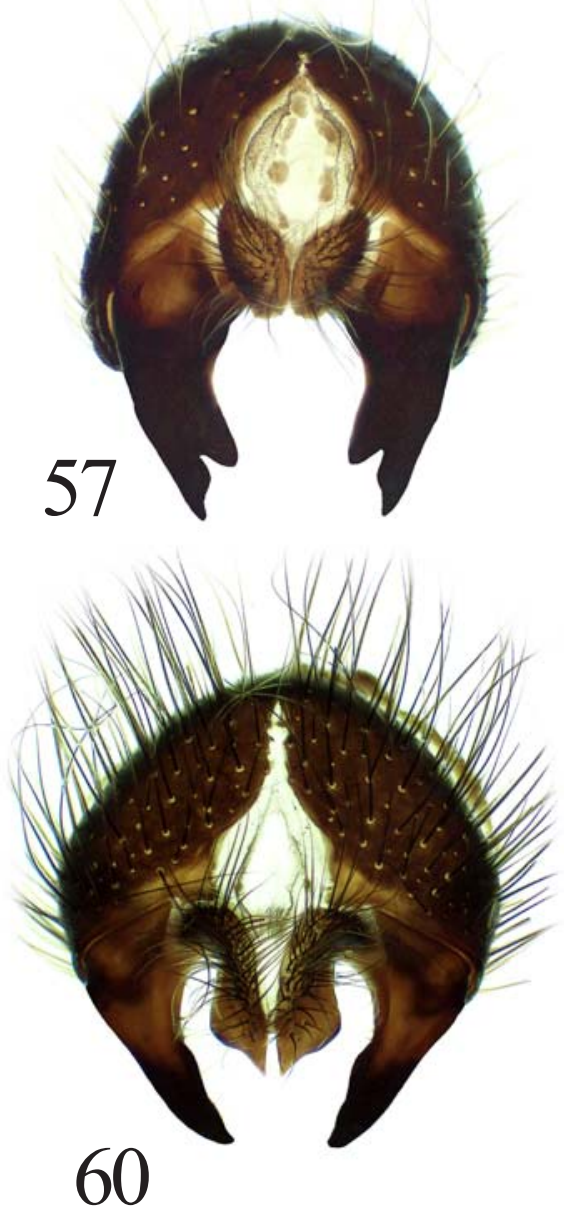

Figs 52-60. Scathophaga cordylurina (Holmgren) (52-54), S crinita (Coquillett) (55-57) and S. dasythrix (Becker) (58-60): 52, 55, 58 - male sternite 5; 53, 56, 59 - epandrium, cercal plate and surstyli, lateral view; 54, 57, 60 - same, dorsal view.

Рис. 52-60. Scathophaga cordylurina (Holmgren) (52-54), S. crinita (Coquillett) (55-57) и S. dasythrix (Becker) (58-60): 52, 55, 58 стернит 5 самца; 53, 56, 59 - эпандрий, церки и сурстили, сбоку; 54, 57, 60 - то же, сверху. 
4 dorsal, $0-1$ posterior, 1 preapical dorsal, and 1 posterior apical setae. Mid femur with 1 preapical anterodorsal seta in male and usually with a row of anterodorsal setae in female, also with 1 preapical posterior and 1 preapical posterodorsal setae in both sexes. Mid tibia with 2-3 posterodorsal, 1 anterodorsal setae and a ring of apicals. Hind femur without conspicuous setae in male and with a row of anterodorsal setae in female. Hind tibia with 3-4 anterodorsal, 2-3 posterodorsal, 1 preapical dorsal, 1 preapical anterodorsal, 1 apical anteroventral setae.

Wing tinged with brownish; crossveins r-m and dmcu darkened (Fig. 9).

Abdomen from yellowish to dark brownish, delicately greyish dusted, covered with not long hairs, tergites with black marginal setulae. Female tergite 7 shiny. Male sternite 4 approximately as long as wide (Fig. 23); sternite 5 with a pair of small narrow apical median processes (Fig. 70). Epandrium, cercal plate and surstyli as in Figs 71, 72.

DISTRIBUTION. Russia: Siberia and Far East from Ural Mountains east to Chukotka, south to Altay, Buryatia, Lake Baikal and Magadan Oblast; — Europe (Finland, Norway); North America.

\section{Scathophaga incompleta Ozerov et Krivosheina, 2020} Figs 24, 73-75, 132.

incompleta Ozerov, Krivosheina, 2020: 228. Type-locality: Priyutnoe env. (46.1 $\left.1^{\circ} \mathrm{N} 43.5^{\circ} \mathrm{E}\right)$ (Russia: Kalmykia).

REMARK. This species was recorded in Russia from Adygea, Crimea and Kalmykia [Ozerov, Krivosheina, 2020: 228].

MATERIAL EXAMINED. Dagestan: Tushilovka $\left(44.310^{\circ} \mathrm{N}\right.$ $\left.46.881^{\circ} \mathrm{E}\right), 12 . X .1921$, Ryabov (1 $\mathrm{O}^{7}$, ZISP). See also Ozerov \& Krivosheina [2020: 228].

ADDITIONAL MATERIAL EXAMINED. Turkmenistan: Etrek $\left(37.658^{\circ} \mathrm{N} 54.763^{\circ} \mathrm{E}\right), 2 . X I .1932$, Ushinskiy (1 $\sigma^{7}$, ZISP) (first record).

DESCRIPTION. Male. Female. Body-length 5.8$6.6 \mathrm{~mm}$.

Head. Frontal vitta yellow, with delicate whitish reflection; fronto-orbital plate blackish, densely pale grey dusted, ocellar triangle blackish. Face and gena yellow, with whitish reflection. Postcranium blackish. 3 orbital and 2-4 frontal setae present. Antenna black; postpedicel about twice as long as wide. Arista bare. Palpus yellow.

Thorax black, densely pale grey dusted; scutum with a double brownish line down the middle, and an obscure one on each side. Acrostichals setulose in two rows, dorsocentrals $2+3$, intra-alars $1+(1-2)$ (posterior postsutural intra-alar seta small, about 0.5 times as long as anterior one or absent), supra-alars $1+2$, postpronotals 2. Scutellum greyish dusted, with a pair of strong basal scutellar and a pair of strong apical scutellar setae.

Legs. All coxae black, greyish dusted. All femora greyish dusted, black, except yellow apex. All tibiae and tarsi yellow. Fore femur with whitish hairs, longer ventrally, with 4-5 dorsal setulae in apical third. Fore tibia with 2-3 dorsal, 3-4 posterodorsal, 1-2 posterior,
1 preapical posterodorsal, 1 apical posteroventral, 1 apical posterior setae. Mid femur with a row of anterodorsal setae, with 1 preapical posterodorsal and 1 preapical posterior setae. Mid tibia with 1-2 anterodorsal, 1-2 posterodorsal, 2 posterior (thin), 1 ventral (strong in female) setae, also with a ring of apicals. Hind femur with a row of dorsal/anterodorsal setae. Hind tibia with 2-3 posterodorsal, 2-3 anterodorsal, 1 preapical dorsal, 1 preapical anterodorsal and 1 apical anteroventral setae.

Wing clear; crossveins $\mathrm{r}-\mathrm{m}$ and dm-cu not darkened.

Abdomen black, densely pale grey dusted, covered with whitish hairs. Tergites 2-5 in male and tergiters 26 in female each with a row of marginal setae. Male sternite 4 almost twice as long as wide (Fig. 24). Male sternite 5 with moderately long and narrow lobes, with a small projection medially between lobes (Fig. 73). Cercal plate longer than surstyli and narrowing to a slender incurved tip (Figs 74, 75). Aedeagus as in Fig. 132.

DISTRIBUTION. Russia: Adygea, Crimea, Dagestan, Kalmykia. - Azerbaijan, Turkmenistan (first record).

\section{Scathophaga inquinata (Meigen, 1826)} Figs 25, 76-78.

inquinata Meigen, 1826: 250 (Scatophaga). Type-locality: not
given.

REMARKS. The species was recorded for Russia from Yaroslavl' Oblast [Ovchinnikov, 2004: 422], Karelia [Humala, Polevoi, 2009: 72] and Mordovia [MacGowan et al., 2021: 17]. Gorodkov [1970: 453] registered this species for Caucasus, but without specifying the place of collecting.

MATERIAL EXAMINED. Altai: Artybash env., floodplain of the River Sema $\left(51.87^{\circ} \mathrm{N}, 87.18^{\circ} \mathrm{E}\right), 550 \mathrm{~m}, 22 . \mathrm{VI} .2009$, V. Sorokina (1 $\sigma^{7}$, ISEA); Biysk $\left(52.535^{\circ} \mathrm{N} 85.178^{\circ} \mathrm{E}\right)$, 5.IX.1971, Sychevskaya ( $1 \sigma^{\top}, 1$ \%, ZISP); Lake Teletskoe, Artybash $\left(51.796^{\circ} \mathrm{N}\right.$, $\left.87.274^{\circ} \mathrm{E}\right), 21 . \mathrm{VI} .1987$, A. Barkalov (1 ○', 4 우, ISEA); Seminsky pass $\left(51.06^{\circ} \mathrm{N} 85.59^{\circ} \mathrm{E}\right), 1650 \mathrm{~m}, 27-30 . \mathrm{VI} .2016$, N. Vikhrev (1 ${ }^{7}$, ZMUM); Seminsky pass, River Sarlyk (51.11 $\left.{ }^{\circ} \mathrm{N} 85.60^{\circ} \mathrm{E}\right), 1200$ m, 28-30.VI.2016, N. Vikhrev (1 $\sigma^{7}$, ZMUM); Seminsky pass, River Turala $\left(50.99^{\circ} \mathrm{N} 85.68^{\circ} \mathrm{E}\right), 1350 \mathrm{~m}, 8^{-12 . V I I .2016, ~ N . ~ V i k h r e v ~}$ (1 $\sigma^{7}$, ZMUM); Tigirek Vill. $\left(51.13^{\circ} \mathrm{N} 83.05^{\circ} \mathrm{E}\right), 550 \mathrm{~m}, 9-$ 12.VII.2014, I. Shamshev (1 $\sigma^{\gamma}, 7$ o⿱, ZISP); Ust-Koksa $\left(50.27^{\circ} \mathrm{N}\right.$ 85.61 $\left.{ }^{\circ} \mathrm{E}\right), 28$. VI.-4.VII.2007, O. Kosterin (1 +, ZMUM); Ust-Sema env., (51.6 $\left.6^{\circ} \mathrm{N} 5.8^{\circ} \mathrm{E}\right), 21-26 . V I .2016, \mathrm{~N}$. Vikhrev (1 ${ }^{\circ}$, ZMUM); Arkhangelsk Oblast: Arkhangelsk $\left(64.546^{\circ} \mathrm{N} 40.567^{\circ} \mathrm{E}\right)$, 4.VIII.2010, D. Gavryushin (1 $\sigma^{\prime}$, ZMUM); Nar'yan-Mar env. $\left(67.631^{\circ} \mathrm{N} 52.985^{\circ} \mathrm{E} ; 67.642^{\circ} \mathrm{N} 53.196^{\circ} \mathrm{E}\right), 6-9$. VII.2008, N.E. Vikhrev, A.L. Ozerov (9 $\Im^{7}$, 5 90 , ZMUM); Nenetsky Nature Reserve, cordon "Bol'shoy Gusinets" (68.175'N 53.645 $\left.{ }^{\circ} \mathrm{E}\right)$, 910.VII.2008, A.L. Ozerov (3 $\sigma^{7} \sigma^{7}$, ZMUM); Solovetskiy I. $\left(65.080^{\circ} \mathrm{N}\right.$ $35.686^{\circ} \mathrm{E}$ ), 26.VII.1975, Gorodkov (1 O $\mathrm{O}^{7}$, ZISP); Solvychegovsk $\left(61.342^{\circ} \mathrm{N} 46.913^{\circ} \mathrm{E}\right), 12$. VIII.2010, D. Gavryushin (1 $\left.\sigma^{7}, \mathrm{ZMUM}\right)$; the lower reaches of the River Pechora $\left(68.334^{\circ} \mathrm{N} 53.304^{\circ} \mathrm{E}\right)$, 11.VII.2008, A.L. Ozerov (1 $\sigma^{7}$, ZMUM); the lower reaches of the River Pechora, Kashin I. $\left(68.242^{\circ} \mathrm{N} 53.856^{\circ} \mathrm{E}\right)$, 10.VII.2008, A.L. Ozerov ( $2 \sigma^{7} \sigma^{7}$, ZMUM); Astrakhan Oblast: Ikryanoe $\left(46.112^{\circ} \mathrm{N}\right.$ $\left.47.767^{\circ} \mathrm{E}\right), 10 . \mathrm{V} .2010$, K. Tomkovich (1 $\sigma^{7}$, ZMUM); Bashkiria: Abzakovo env., River Karan $\left(53.84^{\circ} \mathrm{N} 58.566^{\circ} \mathrm{E}\right), 551 \mathrm{~m}$, 17.VII.2015, D. Gavryushin (1 $\sigma^{7}$, ZMUM); Bashkirskiy Nature Reserve $\left(53.420^{\circ} \mathrm{N} 57.935^{\circ} \mathrm{E}\right)$, 7.VIII.1975, Gorodkov (3 $\sigma^{7} \sigma^{7}$, ZISP); Levaya Belaya Station $\left(54.708^{\circ} \mathrm{N} 55.885^{\circ} \mathrm{E}\right), 18 . \mathrm{VIII} .1975$, Gorodkov (2 $\left.\sigma^{\top} \sigma^{\top}, \operatorname{ZISP}\right)$; Urman $\left(54.878^{\circ} \mathrm{N} 56.877^{\circ} \mathrm{E}\right)$, 
19.VIII.1975, Gorodkov ( 1 , ZISP); Karachay-Cherkessia: Dombay, Valley of the River Alibek $\left(43.291^{\circ} \mathrm{N} 41.594^{\circ} \mathrm{E}\right), 2500 \mathrm{~m}$, 1.VII.1982, Nartshuk (1 $\sigma^{7}$, ZISP); Karelia: Primorsky env. $\left(66.549^{\circ} \mathrm{N} 33.133^{\circ} \mathrm{E}, 66.555^{\circ} \mathrm{N} 33.006^{\circ} \mathrm{E}, 66.552^{\circ} \mathrm{N} 33.100^{\circ} \mathrm{E}\right), 3-$ 5.VII.2010, A.L. Ozerov (3 $\sigma^{7} \sigma^{7}$, ZMUM); Krasnodar Krai: Agurskoe gorge $\left(43.548^{\circ} \mathrm{N} 39.814^{\circ} \mathrm{E}\right), 25 . I V .2008$, A.L. Ozerov (1 9 , ZMUM); Lagonaki (44.009 $\left.\mathrm{N} 39.994^{\circ} \mathrm{E}\right), 1700 \mathrm{~m}, 11-12 . \mathrm{VII} .2008$, O. Kosterin ( 1 , $\mathrm{ZMUM})$; Lagonaki $\left(44.01^{\circ} \mathrm{N} 40.02^{\circ} \mathrm{E}\right), 1500$ 1700 m, 5-7.V.2013, N. Vikhrev (3 $\sigma^{7} \sigma^{7}$, ZMUM); Lagonaki $\left(44.107^{\circ} \mathrm{N} 40.020^{\circ} \mathrm{E}, 1450 \mathrm{~m} ; 44.093^{\circ} \mathrm{N} 40.019^{\circ} \mathrm{E}, 1725 \mathrm{~m}\right), 15-17$ and 26-28.VI.2009, K. Tomkovich (6 $\sigma^{7} \sigma^{7}$, ZMUM); Lakes Khmelevskie $\left(43.716^{\circ} \mathrm{N} 40.201^{\circ} \mathrm{E}\right), 10 . \mathrm{VII} .2008$, O. Kosterin $\left(1 \sigma^{7}\right.$ ZMUM); Sochi, Estosadok env. (43.691 $\left.{ }^{\circ} \mathrm{N} 40.366^{\circ} \mathrm{E}\right), 20$ 22.VI.2008, K. Tomkovich (3 $\sigma^{7} \sigma^{7}$, ZMUM); Sochi, Mt. Akhun $\left(43.523^{\circ} \mathrm{N} 39.879^{\circ} \mathrm{E}\right), 22 . X .2006$, N. Vikhrev (2 $\sigma^{\top} \sigma^{7}$, ZMUM); Sochi/Adler $\left(43.476^{\circ} \mathrm{N} 39.906^{\circ} \mathrm{E}\right), 21 . X .2010$, D. Gavryushin (1 $\sigma^{7}, 1$ ㅇ, ZMUM); Sochi/Khosta $\left(43.523^{\circ} \mathrm{N} 39.879^{\circ} \mathrm{E}\right), 15 . \mathrm{V} .2011$, D. Gavryushin (1 $\sigma^{7}$, ZMUM); Teberdinskiy Nature Reserve, Valley of the River Teberda $\left(43.405^{\circ} \mathrm{N} 41.721^{\circ} \mathrm{E}\right), 11$. VII. 1982 , Nartshuk (1 $\sigma^{7}$, ZISP); Vostochnoe $\left(53.573^{\circ} \mathrm{N} 92.128^{\circ} \mathrm{E}\right)$, 19.V.1923, Zhelokhovtsev (1 O, ZMUM); Arkhyz $\left(43.564^{\circ} \mathrm{N}\right.$ $\left.41.280^{\circ} \mathrm{E}\right), 1450 \mathrm{~m}, 12 . \mathrm{IX} .1965$, Gorodkov (1 $\sigma^{7}$, ZISP); Karachaevsk $\left(43.778^{\circ} \mathrm{N} 41.915^{\circ} \mathrm{E}\right), 900 \mathrm{~m}, 26 . I V .1964$, Gorodkov (1 ح", ZISP); Teberdinskiy Nature Reserve $\left(43.405^{\circ} \mathrm{N} 41.721^{\circ} \mathrm{E}\right), 25 . \mathrm{IV}$ and 3.VI.1964, Gorodkov (3 $\sigma^{\top} \sigma^{7}$, ZISP); Krasnoyarsk Krai: National Park "Stolby" $\left(55.887^{\circ} \mathrm{N} 92.817^{\circ} \mathrm{E}\right), 29$. VIII.1973, Gorodkov (1 $\sigma^{7}$, ZISP); Kursk Oblast: Oboyan' $\left(51.191^{\circ} \mathrm{N} 36.312^{\circ} \mathrm{E}\right)$ 20-21.VII.2007, A.L. Ozerov (1 $\sigma^{7}$, ZMUM); Leningrad Oblast: Luga (ca. $58.73^{\circ} \mathrm{N} 29.84^{\circ} \mathrm{E}$ ), 11.V.1954, 27.VI.1955, A. Stackel-

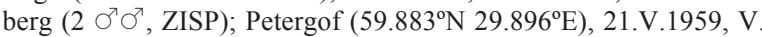
Sychevskaya (2 $\sigma^{7} \mathrm{O}^{7}$, ZMUM); Rakovichi $\left(58.650^{\circ} \mathrm{N} 29.836^{\circ} \mathrm{E}\right)$, 15.VI. and 1.IX.1897, Pleske ( $1 \sigma^{7}, 1$ †, ZISP); Saint Petersburg $\left(59.934^{\circ} \mathrm{N} 30.359^{\circ} \mathrm{E}\right), 29 . \mathrm{VIII} .1995$, Gorodkov (6 o $\sigma^{\top}, 1$ ㅇ, ZISP); Saint Petersburg $\left(59.934^{\circ} \mathrm{N} 30.359^{\circ} \mathrm{E}\right), 31$. VII.1995, Gorodkov (1 $\sigma^{7}$, ZISP); Sergievka $\left(59.893^{\circ} \mathrm{N} 29.839^{\circ} \mathrm{E}\right), 25 . \mathrm{IX} .1930$, Burakova (5 $\sigma^{\prime} \sigma^{7}$, ZISP); Shuvalovo $\left(60.047^{\circ} \mathrm{N} 30.283^{\circ} \mathrm{E}\right), 27 . V I .1897$, Yakobson (1 $\sigma^{7}$, ZISP); same place, 19.V.1954, Razhev (2 $\sigma^{7} \sigma^{7}$, ZISP); Tolmachevo $\left(58.856^{\circ} \mathrm{N} 29.895^{\circ} \mathrm{E}\right)$, 7.VIII.1935, Rohdendorf (1 0 ZISP); Moscow and Moscow Oblast: Abramtsevo $\left(56.23^{\circ} \mathrm{N}\right.$ $\left.37.956^{\circ} \mathrm{E}\right)$, 31.V.1958, 16.VI.1959, E.S. Smirnov (2 우, ZMUM); Andreevskoe $\left(55.967^{\circ} \mathrm{N} 35.609^{\circ} \mathrm{E}, 55.978^{\circ} \mathrm{N} 35.588^{\circ} \mathrm{E}\right), 28 . \mathrm{IV}$. and 14.V.2007, 6.V.2009, A.L. Ozerov (4 $\sigma^{7} \sigma^{7}, 1$ 9, ZMUM); Bol'shevo $\left(55.925^{\circ} \mathrm{N} 37.868^{\circ} \mathrm{E}\right), 7 . V .1930, \mathrm{~N}$. Violovich (1 $\sigma^{\top}, 1$ O, ZMUM) Burtsevo $\left(55.981^{\circ} \mathrm{N} 35.597^{\circ} \mathrm{E}, 55.975^{\circ} \mathrm{N} 35.586^{\circ} \mathrm{E}\right), 2-3 . \mathrm{VI} .2006$, 10.X.2010, A.L. Ozerov (2 $\sigma^{7} \sigma^{7}$, ZMUM); Dmitrov env. $\left(56.316^{\circ} \mathrm{N}\right.$ $\left.37.725^{\circ} \mathrm{E}\right), 16$.V. and 21.VI.2007, 10.V.2008, N. Vikhrev $\left(2 \sigma^{7} \sigma^{7}, 2\right.$ $\mathrm{O}$, ZMUM); Golitsyno $\left(55.649^{\circ} \mathrm{N} 37.011^{\circ} \mathrm{E}\right), 12,13$ and 17.V.1979, 17 and 24.V.1981, 26.VI.1982, A. Shatalkin (1 ○', 5 우, ZMUM); Izmaylovo $\left(55.786^{\circ} \mathrm{N} 37.835^{\circ} \mathrm{E}\right), 15 . \mathrm{V} .1983,3$ and $29 . \mathrm{V} .1986$, A.L.

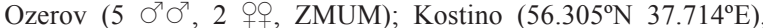
7.V.2010, D. Gavryushin (1 $\mathrm{O}^{7}$, ZMUM); Luzhki $\left(55.833^{\circ} \mathrm{N}\right.$ $\left.37.550^{\circ} \mathrm{E}\right)$, 29.IV.2000, A. Gusakov (1 $\sigma^{7}$, ZMUM); Molzhaninovka $\left(55.936^{\circ} \mathrm{N} 37.385^{\circ} \mathrm{E}\right)$, 13.V.2010, A.L. Ozerov (1 $\sigma^{7}$, ZMUM); Ozhigovo $\left(55.455^{\circ} \mathrm{N} 36.882^{\circ} \mathrm{E}\right), 27 . I V .2008,9 . V .2009$, D. Gavryushin (2 $\sigma^{7} \sigma^{\top}, 1$, 1 ZMUM); Podol'sk $\left(45.448^{\circ} \mathrm{N} 37.563^{\circ} \mathrm{E}\right), 10-15 . I V .2010$, K. Tomkovich (1 $\sigma^{7}$, ZMUM); Pripuschaevo $\left(56.702^{\circ} \mathrm{N} 37.681^{\circ} \mathrm{E}\right)$, 8-10.IX.2008, K. Tomkovich (2 $\sigma^{\top} \sigma^{7}$, ZMUM); Serpukhov env. $\left(54.924^{\circ} \mathrm{N} 37.409^{\circ} \mathrm{E}\right), 1 . X .1901$, G.A. Kozhevnikov (1 ${ }^{7}$, ZMUM) Shchukino $\left(55.798^{\circ} \mathrm{N} 37.478^{\circ} \mathrm{E}\right), 19 . \mathrm{IX} .2006$, N. Vikhrev (1 O ZMUM); Zelenograd $\left(55.986^{\circ} \mathrm{N} 37.202^{\circ} \mathrm{E}\right), 19 . \mathrm{VI} .1998,15 . \mathrm{V} .1999$, A. Gusakov (2 $\sigma^{7} \sigma^{7}, 1$ ․, ZMUM); Zvenigorod env. $\left(55.700^{\circ} \mathrm{N}\right.$ $\left.36.722^{\circ} \mathrm{E}\right)$, 24.V.1981, 1-4.V.1996, A.L. Ozerov, K. Tomkovich (12 $\sigma^{7}, 1$ O , ZMUM); Nizhegorod Oblast: Dzerzhinsk $\left(56.21^{\circ} \mathrm{N}\right.$ 43.62 $\left.{ }^{\circ} \mathrm{E}\right), 18$.VIII.2009, N. Vikhrev (1 $\sigma^{7}$, ZMUM); North Ossetia Alania: Alagir env. $\left(43.015^{\circ} \mathrm{N} 44.224^{\circ} \mathrm{E}\right), 18$ and 19.V.1989, A.L. Ozerov (1 $\sigma^{\top}, 2$ 우 ZMUM); Bakhty-Lapparyrag ridge $\left(42.938^{\circ} \mathrm{N}\right.$ 44.287 $\left.{ }^{\circ} \mathrm{E}\right), 1770 \mathrm{~m}, 18 . V ., 1$ and 13.VI.1989, 29-30.VI.1990, A.L.

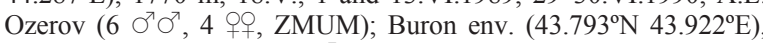
7.VI.1989, A.L. Ozerov (1 $\sigma^{7}$, ZMUM); Tsey $\left(43.793^{\circ} \mathrm{N} 43.922^{\circ} \mathrm{E}\right)$ 16.IX.1989, A. Shatalkin ( 2 + , ZMUM); Novgorod Oblast: Malaya Vishera $\left(58.849^{\circ} \mathrm{N} 32.209^{\circ} \mathrm{E}\right), 9 . V .1975$, Gorodkov $\left(2 \sigma^{\top} \sigma^{7}, 2\right.$ 우, ZISP); Spas-Nereditsa $\left(58.497^{\circ} \mathrm{N} 31.312^{\circ} \mathrm{E}\right), 22$.VI.1975, Gorodkov $\left(1 \sigma^{\top}, 1\right.$, ZISP); Novosibirsk Oblast: Orlovka env. $\left(59.93^{\circ} \mathrm{N}\right.$

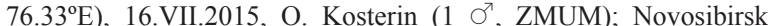
$\left(54.842^{\circ} \mathrm{N} 83.114^{\circ} \mathrm{E}\right), 9-10 . I X .2009$, O. Kosterin (2 $\mathrm{O}^{\top} \sigma^{\top}$, ZMUM); Novosibirsk $\left(55.52^{\circ} \mathrm{N} 83.24^{\circ} \mathrm{E}\right), 22 . V .2011$, O. Kosterin $\left(1 \sigma^{\prime}\right.$, ZMUM); Academgorodok $\left(54.87^{\circ} \mathrm{N} 83.05^{\circ} \mathrm{E}\right), 18-19 . \mathrm{VI} .2016, \mathrm{~N}$. Vikhrev (1 $\sigma^{7}$, ZMUM); Pskov Oblast: "Pokrovskaya Novor[zhevskogo] u[ezda] Pskov[skoy] g[ubernii]" $\left(57.242^{\circ} \mathrm{N}\right.$ $\left.28.431^{\circ} \mathrm{E}\right), 15$.VII.1915, N. Kuznetsov (1 9 , ZISP); Rostov Oblast: Taganrog $\left(47.226^{\circ} \mathrm{N} 38.898^{\circ} \mathrm{E}\right), 6$ and 8.IX.1927, C. Ahnger $\left(3 \sigma^{\top} \sigma^{\top}\right.$, ZISP); Ryazan' Oblast: Gremyachka $\left(53.487^{\circ} \mathrm{N} 39.515^{\circ} \mathrm{E}\right)$, 5.IX.1904, A. Semenov (1 9 , ZISP); Zalesnoe $\left(54.969^{\circ} \mathrm{N} 41.327^{\circ} \mathrm{E}\right)$, 21-26.VII.2013, N. Vikhrev (1 ๑ , ZMUM); Saratov Oblast: Saratov env. $\left(51.558^{\circ} \mathrm{N} 46.018^{\circ} \mathrm{E}\right)$, 5.VIII.1917, A. Stackelberg (1 フ', ZISP); Smolensk Oblast: National Park "Smolenskoe poozer'e" $\left(55.511^{\circ} \mathrm{N} 31.839^{\circ} \mathrm{E}\right), 24$.VII., 3-11.VIII.1991, Zlobin (3 $\sigma^{7} \sigma^{7}, 1$ 우, ZISP); Tver' Oblast: Boglaevo $\left(58.155^{\circ} \mathrm{N} 34.797^{\circ} \mathrm{E}\right), 8-31 . X .2009$, A.G. Korobkov (1 $\sigma^{T}$, ZISP); Tyumen' Oblast: Novyy Urengoy $\left(66.087^{\circ} \mathrm{N} 76.513^{\circ} \mathrm{E}\right), 30 . \mathrm{VII} .1982$, Gorodkov (1 $\sigma^{7}$, ZISP); Salekhard $\left(66.53^{\circ} \mathrm{N} 66.6135^{\circ} \mathrm{E}\right), 3$. VIII.1961, Gorodkov (1 $\sigma^{7}$, ZISP); Ul'yanovsk Oblast: Radishchevo $\left(55.836^{\circ} \mathrm{N} 48.367^{\circ} \mathrm{E}\right), 2-5 . \mathrm{V} .2011$, K. Tomkovich (1 $\sigma^{7}$, ZMUM); Volgograd Oblast: Serafimovich env. (49.5 $\left.7 \mathrm{~N} 42.6^{\circ} \mathrm{E}\right), 28-29 . I V .2013$, N. Vikhrev (1 フ, ZMUM); Vologda Oblast: Belozersk env. (60.029 $\left.\mathrm{N} 37.791^{\circ} \mathrm{E}\right), 13 . \mathrm{VI} .1964$, Borodin $\left(2 \circ \circ\right.$, ZMUM); Vorohezh Oblast: Voronezh env. $\left(51.695^{\circ} \mathrm{N}\right.$ $\left.39.151^{\circ} \mathrm{E}\right)$, 3.V.2012, Kornev (1 $\sigma^{7}$, ZISP); Voronezh env., Shilovskiy Forest $\left(51.601^{\circ} \mathrm{N} 39.181^{\circ} \mathrm{E}\right)$, 7.IX.1965, Negrobov $\left(2 \sigma^{\top} \sigma^{7}, 1\right.$ +, ZISP); Yaroslavl' Oblast: Yaroslavl' $\left(57.632^{\circ} \mathrm{N} 39.836^{\circ} \mathrm{E}\right)$, 1.VI.1988, M.A. Klepikov (1 \%', ZISP).

DESCRIPTION. Male. Female. Body-length 4.9$8.8 \mathrm{~mm}$.

Head. Frontal vitta yellow or reddish-yellow, matt; fronto-orbital plate blackish, greyish dusted. Face, parafacial and gena yellow, with delicate golden reflection. Postcranium black in upper third or half and yellow in lower part. 3 orbital and 4-5 frontal setae present. Antenna reddish-yellow. Postpedicel approximately twice as long as wide. Arista pubescent, the longest hairs approximately equal to $1 / 3-1 / 2$ width of postpedicel. Palpus yellow.

Thorax black in ground colour, greyish dusted, but usually postpronotal lobe yellowish. Acrostichals not differentiated from the other hairs on scutum or setulose in two rows, but prescutellar pair usually stronger than the other acrostichals, dorsocentrals $2+3$, intraalars $1+2$, supra-alars $1+2$, postpronotals 2 . Katepisternum posteriorly with yellow hairs and setulae, but without black setulae. Anepimeron bare. Scutellum black, greyish dusted, with a pair of strong basal scutellar and a pair of strong apical scutellar setae.

Legs yellow. Fore femur covered with hairs, but without conspicuous setae. Fore tibia with 2 dorsal, 0 1 posterior, 1 preapical dorsal, and 1 posterior apical setae. Mid femur with a row of anterior/anterodorsal setae, also with 1 preapical posterior and 1 preapical posterodorsal setae in both sexes. Mid tibia with 2-3 posterodorsal, 1 anterodorsal setae and a ring of apicals. Hind femur with a row of anterodorsal setae in both sexes. Hind tibia with 3 anterodorsal, 3 posterodorsal, 1 preapical dorsal, 1 preapical anterodorsal, 1 apical anteroventral setae.

Wing tinged with brownish; crossveins r-m and dmcu darkened.

Abdomen from yellow to dark brownish, delicately greyish dusted, covered with not long hairs, in female tergites with black marginal setulae. 


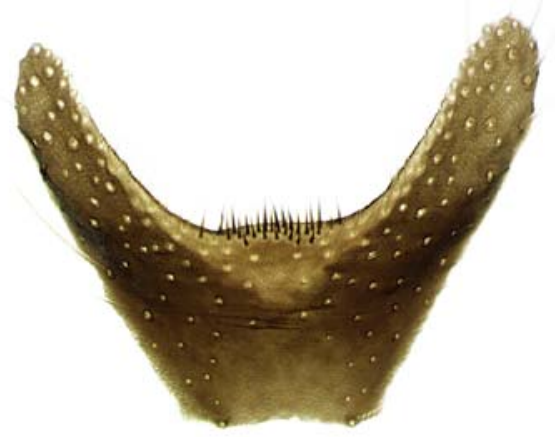

61
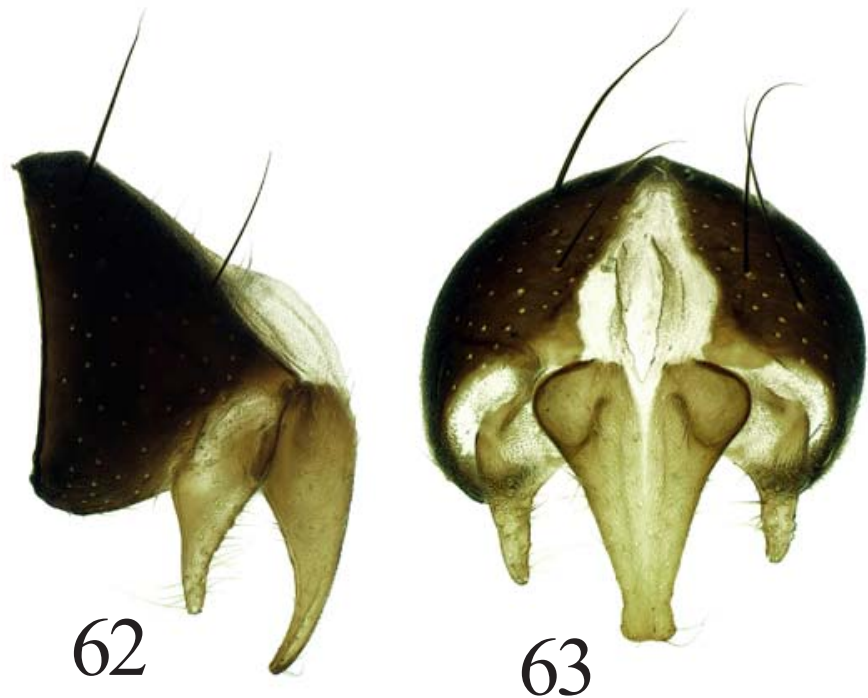

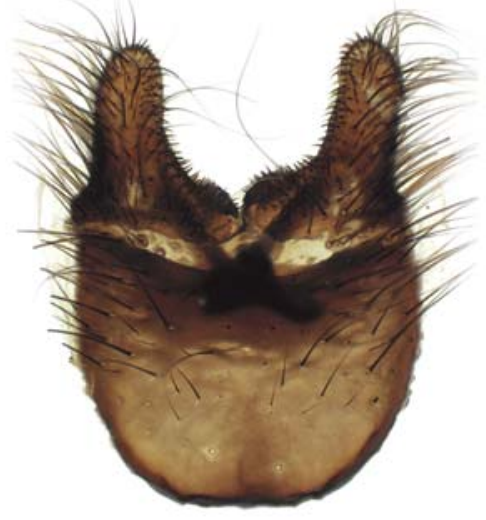

64

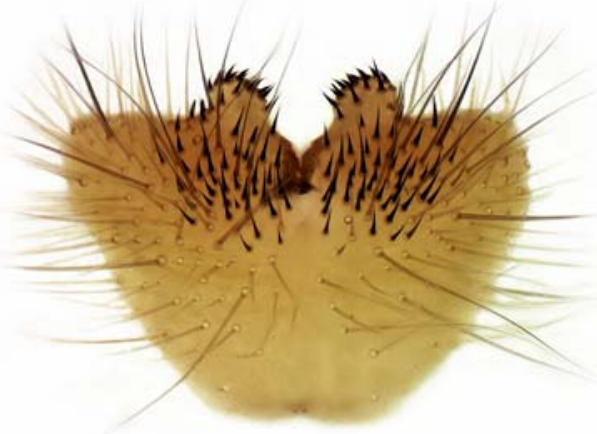

67
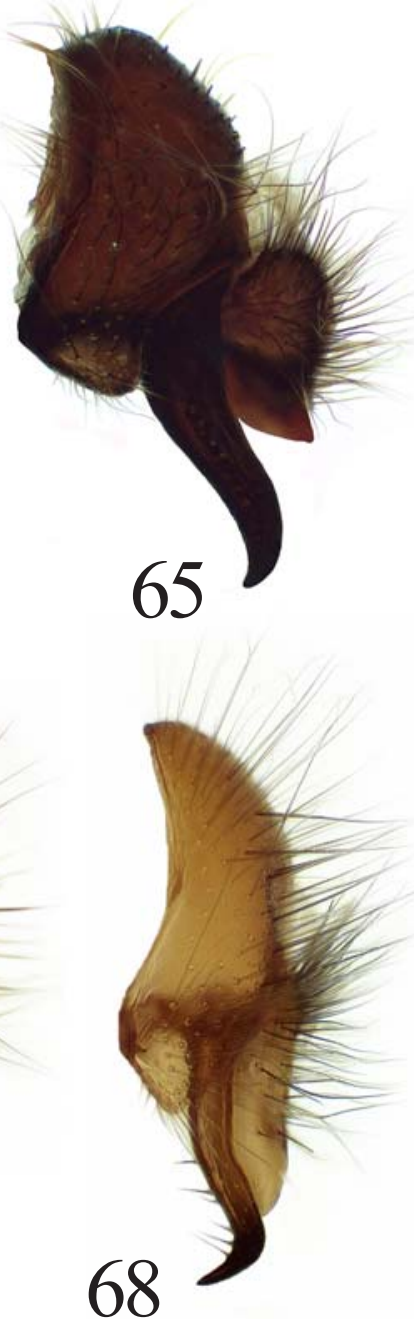

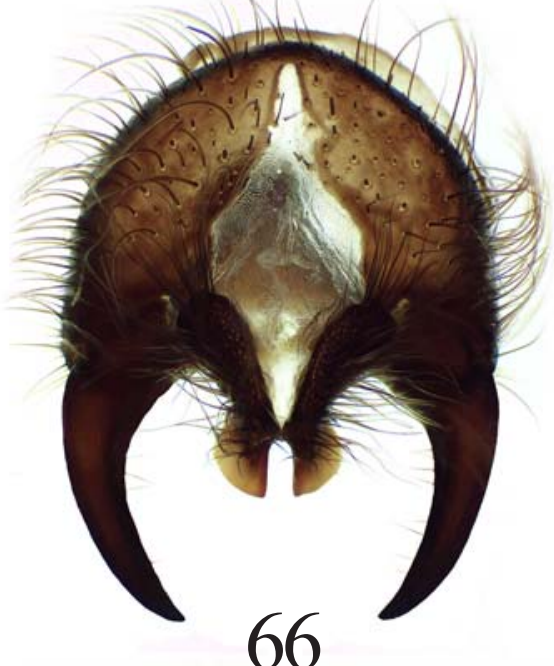

66

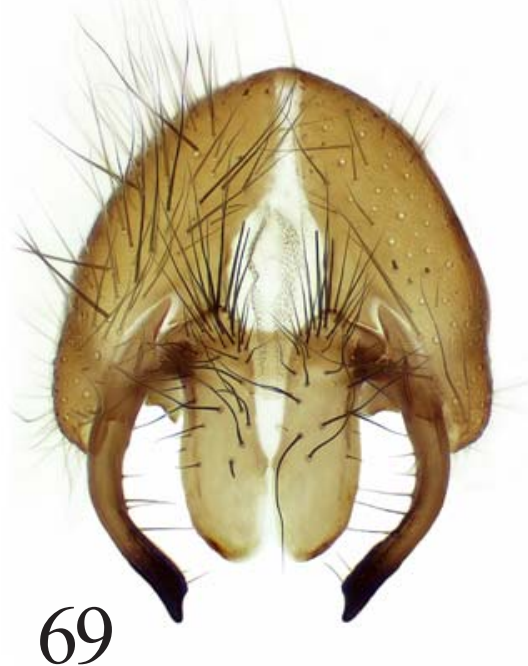

Figs 61-69. Scathophaga decipiens (Haliday) (61-63), S. exalata Ozerov (64-66) and S. furcata (Say) (67-69): 61, 64, 67 - male sternite 5; 62, 65, 68 - epandrium, cercal plate and surstyli, lateral view; 63, 66, 69 - same, dorsal view. 61-63 — after Ozerov \& Krivosheina, 2020, figs 4, 6, 7.

Pис. 61-69. Scathophaga decipiens (Haliday) (61-63), S. exalata Ozerov (64-66) и S. furcata (Say) (67-69): 61, 64, 67 - стернит 5 самца; 62, 65, 68 - эпандрий, церки и сурстили, сбоку; 63, 66, 69 - то же, сверху. 61-63 - after Ozerov \& Krivosheina, 2020, figs 4, 6, 7. 


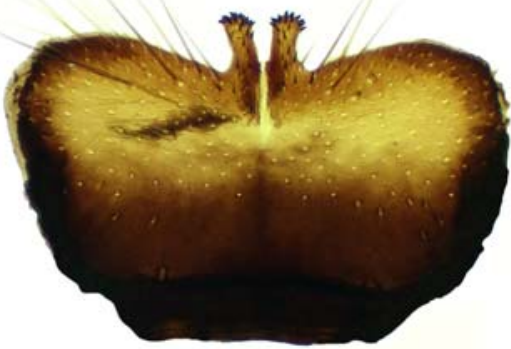

70

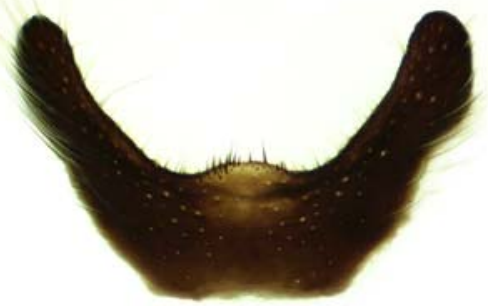

73

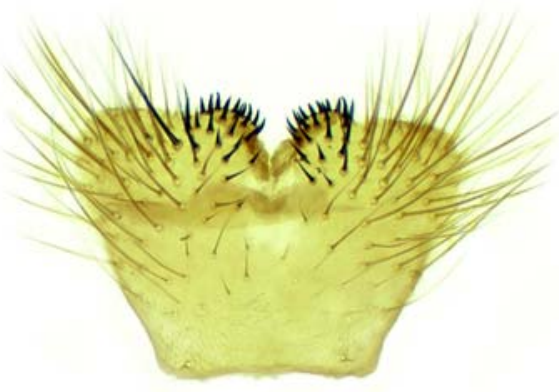

76

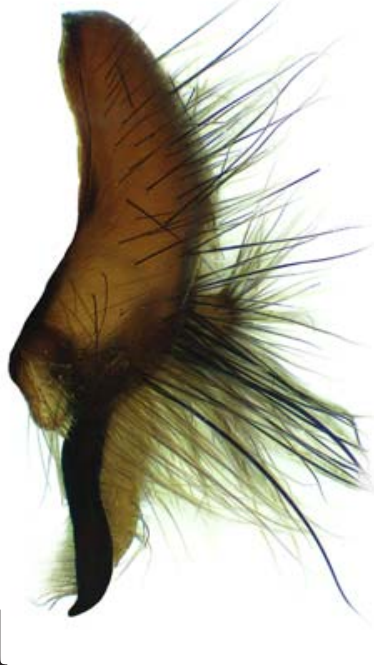

71

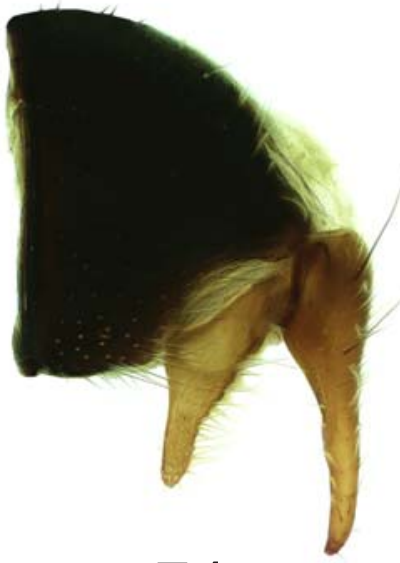

74

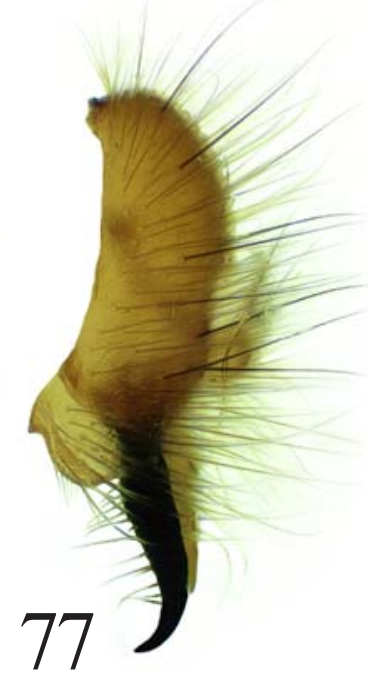

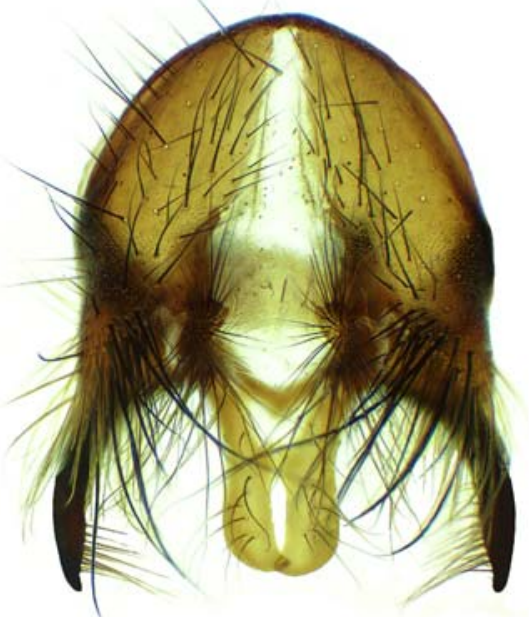

72

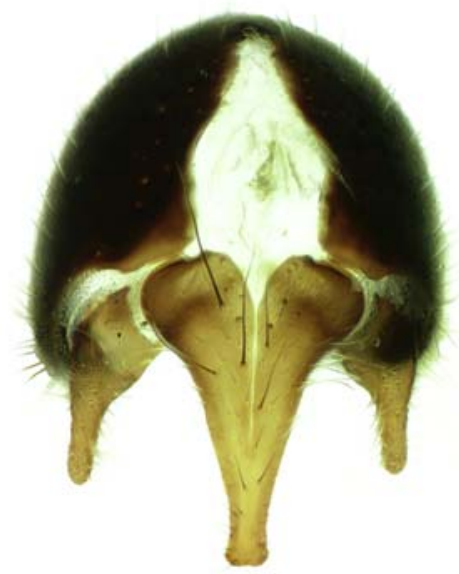

75

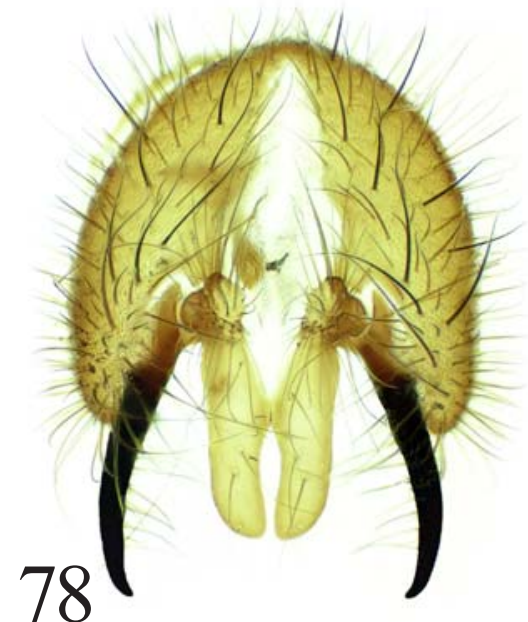

Figs 70-78. Scathophaga incola (Becker) (70-72), S. incompleta Ozerov et Krivosheina (73-75) and S. inquinata (Meigen) (76-78): 70, 73, 76 - male sternite 5; 71, 74, 77 — epandrium, cercal plate and surstyli, lateral view; 72, 75, 78 - same, dorsal view. 73-75 after Ozerov \& Krivosheina, 2020, figs 10, 12, 13.

Рис. 70-78. Scathophaga incola (Becker) (70-72), S. incompleta Ozerov et Krivosheina (73-75) и S. inquinata (Meigen) (76-78): 70, 73, 76 - стернит 5 самца; 71, 74, 77 — эпандрий, церки и сурстили, сбоку; 72, 75, 78 - то же, сверху. 73-75 — по Оzеrov \& Krivosheina, 2020, figs 10, 12, 13. 


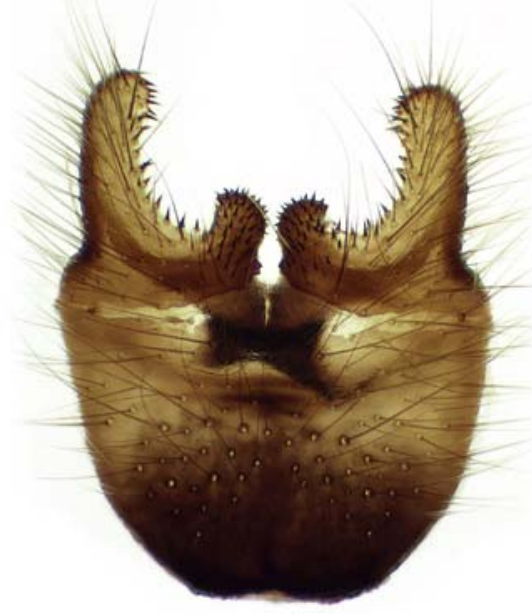

79

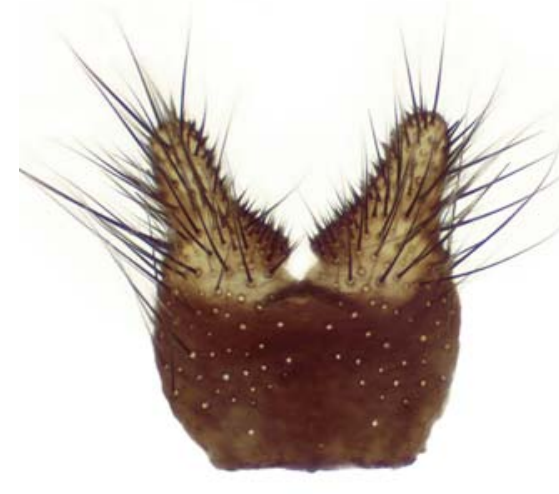

82

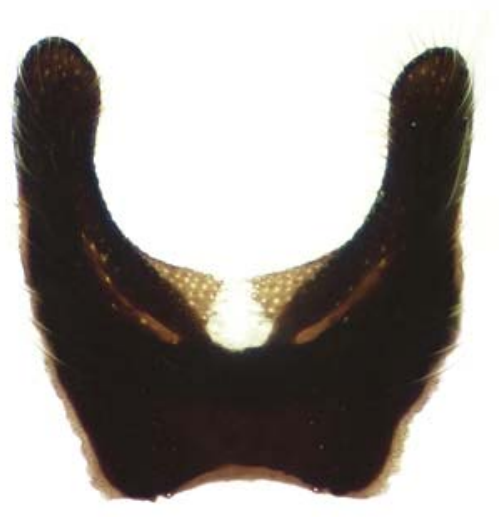

85
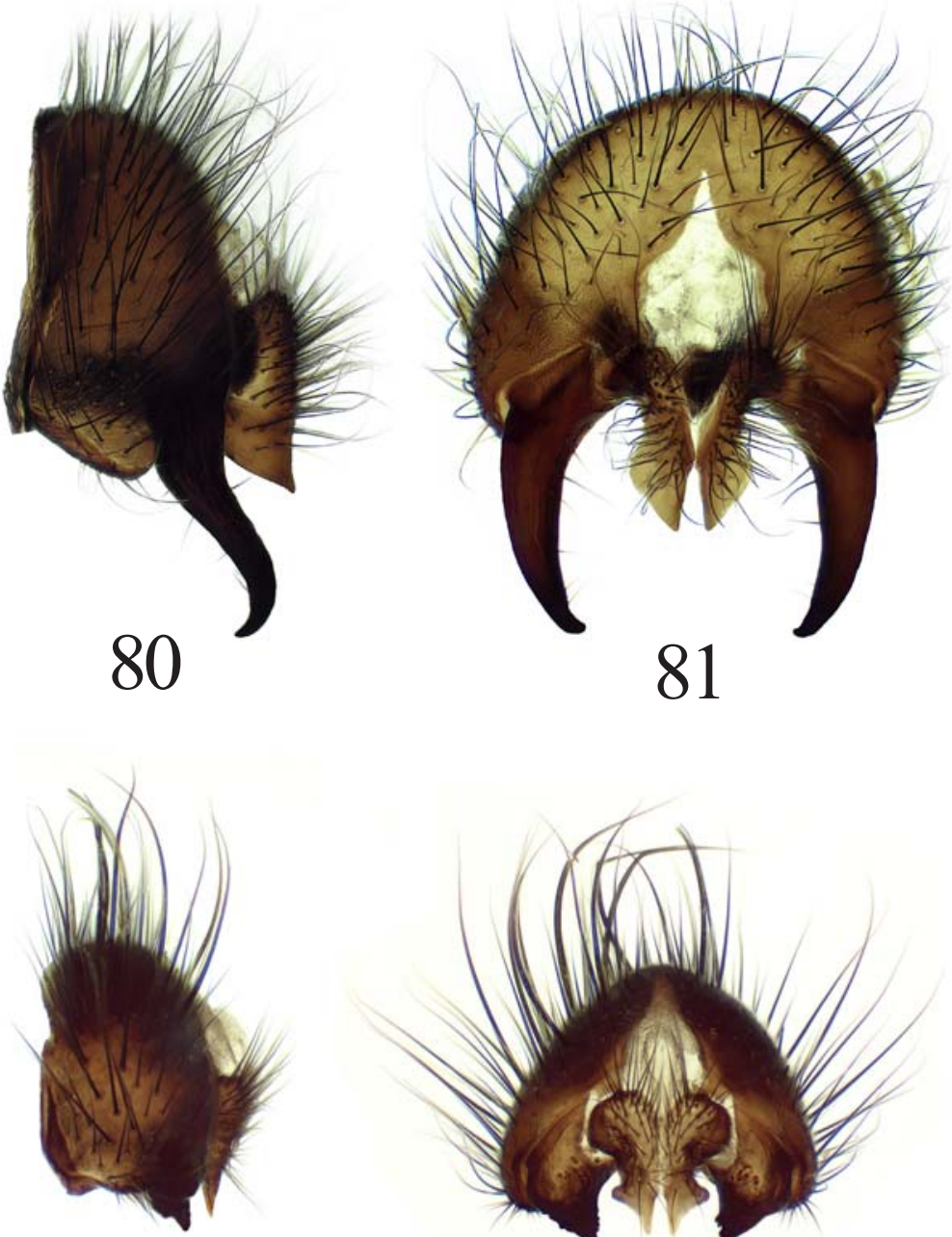

83

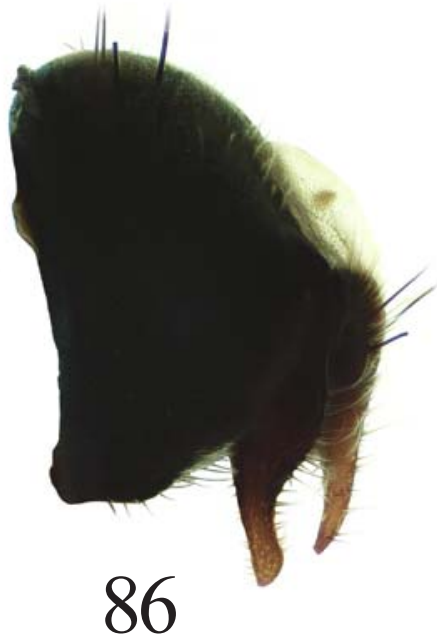

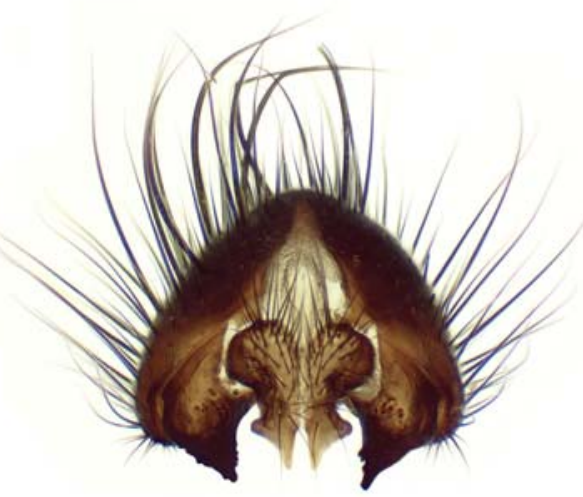

84

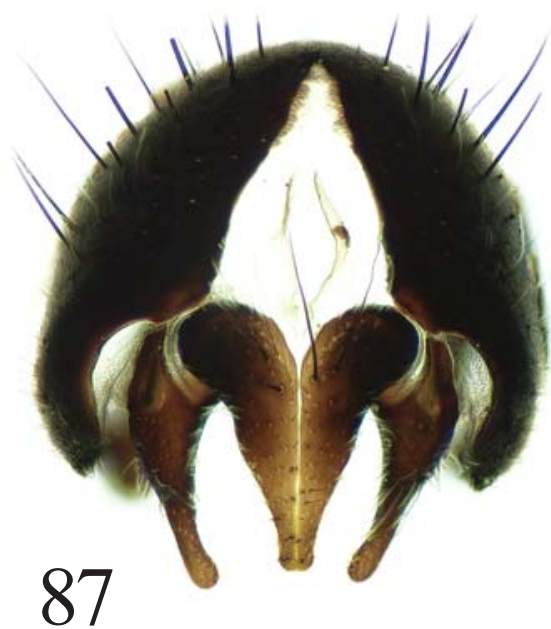

Figs 79-87. Scathophaga intermedia (Walker) (79-81), S. karelica Ozerov (82-84) and S. lapponica (Ringdahl) (85-87): 79, 82, 85 - male sternite 5; 80, 83, 86 - epandrium, cercal plate and surstyli, lateral view; 81, 84, 87 — same, dorsal view. 82-84 — after Ozerov, 2013, figs $14-16$.

Рис. 79-87. Scathophaga intermedia (Walker) (79-81), S. karelica Ozerov (82-84) и S. lapponica (Ringdahl) (85-87): 79, 82, 85 стернит 5 самца; 80, 83, 86 - эпандрий, церки и сурстили, сбоку; 81, 84, 87 — то же, сверху. 82-84 — по Ozerov, 2013, figs $14-16$. 
Male sternite 4 approximately 2 times as long as wide (Fig. 25); sternite 5 with a pair of very small apical median processes, covered with spinules (Fig. 76). Epandrium, cercal plate and surstyli as in Figs 77, 78.

DISTRIBUTION. Russia: forest zone of European part and Siberia. - Europe (widespread). Common.

Scathophaga intermedia (Walker, 1849) Figs 26, 79-81.

intermedia Walker, 1849: 980 (Scatophaga). Type-locality: "Nova Scotia" (Canada).

hadleyi Ozerov, 2013: 88 (Scathophaga). Type-locality: Tserkovnaya Bay $\left(43.75^{\circ} \mathrm{N} 146.70^{\circ} \mathrm{E}\right)$, Shikotan I. (Russia).

REMARK. The species was registered for Russia from the Far East by Gorodkov [1986: 31, on "sea coast", but without specifying the place of collecting] and by Ozerov \& Krivosheina [2014: 217].

MATERIAL EXAMINED. Kamchatka Krai: Apuka env. $\left(60.443^{\circ} \mathrm{N} 169.602^{\circ} \mathrm{E}\right), 19 . \mathrm{VIII} .1959$, Gorodkov (3 oㅇ, ZISP); Commander Islands, Mednyy I. (ca $\left.54.790^{\circ} \mathrm{N} 167.578^{\circ} \mathrm{E}\right), 29 . \mathrm{VII}$., VII.-VIII.1932, I. Barabash (2 $\sigma^{7} \sigma^{7}, 1$ ㅇ, ZISP); Commander Islands, Nikol'skoe $\left(55.196^{\circ} \mathrm{N} 165.998^{\circ} \mathrm{E}\right)$, 9.IX.1959, Gorodkov (6 $\sigma^{\top} \sigma^{\top}, 5$ 우, ZISP); Commander Islands, Preobrazhenskoe

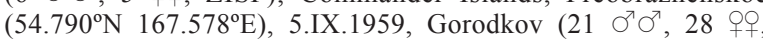
ZISP); Nizhne-Kamchat.[sky ostrog] $\left(56.247^{\circ} \mathrm{N} 162.017^{\circ} \mathrm{E}\right)$, 15.VIII.1909, P. Shmidt (1 9 , ZISP); Ust'-Kamchatsk $\left(56.229^{\circ} \mathrm{N}\right.$ $\left.162.469^{\circ} \mathrm{E}\right), 10$.VIII.1930, E. Gur'yanova (1 $\sigma^{7}$, ZISP); Khabarovsk Krai: Bulgino env. (59.371 $\left.{ }^{\circ} \mathrm{N} 143.147^{\circ} \mathrm{E}\right)$, 29.VIII.1987, Gorodkov (1 ${ }^{\top}$, ZISP); Imperatorskaya Gavan’ [=

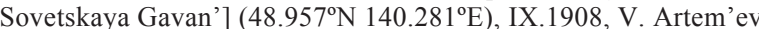
(8 $\sigma^{7} \sigma^{7}, 2$ 우, ZISP); Cape Pronge $\left(52.853^{\circ} \mathrm{N} 141.225^{\circ} \mathrm{E}\right), 10$ 11.IX.1911, Derbek ( $7 \sigma^{7} \sigma^{7}, 6$ 우, ZISP); Magadan Oblast: Evensk $\left(61.916^{\circ} \mathrm{N} 159.234^{\circ} \mathrm{E}\right)$, 8.IX.1987, Gorodkov (10 $\sigma^{7} \sigma^{7}, 3$ 우, ZISP); Koni peninsula, cordon "Mys Plosky" $\left(59.15^{\circ} \mathrm{N}\right.$

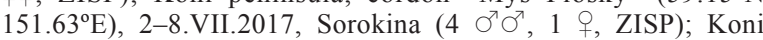
peninsula, Cape Plosky $\left(59.161^{\circ} \mathrm{N} 151.643^{\circ} \mathrm{E}\right), 16 . \mathrm{VII}$. and 10.IX.2015, 21.VIII.2017, N. Tridrikh (35 $\sigma^{7} \sigma^{7}, 12$ oᄋ, ZMUM); Sakhalin Oblast: Iturup I., Rybaki $\left(45.209^{\circ} \mathrm{N} 147.851^{\circ} \mathrm{E}\right)$, 23.VI.1968, V. Richter (1 ○', 1 क, ZISP); Kunashir I., Dubovoe $\left(43.766^{\circ} \mathrm{N} 145.499^{\circ} \mathrm{E}\right), 10 . \mathrm{VI} .1973,20$. VII.1973, Kerzhner $(2$ 우, ZISP); Kunashir I., Golovnino $\left(43.737^{\circ} \mathrm{N} 145.517^{\circ} \mathrm{E}\right)$, 27.IX.1968, Gorodkov (1 $\circ$, ZISP); Paramushir I., Severo-Kuril'sk $\left(50.679^{\circ} \mathrm{N}\right.$ $\left.156.132^{\circ} \mathrm{E}\right), 7-12 . I X .1968$, Gorodkov (28 $\sigma^{7} \sigma^{7}, 22$ of, ZISP); same place, 18.VIII.1978, A. Zinov'ev (28 $\sigma^{\top} \sigma^{7}, 22$ 우, ZISP); Sakhalin I., Rakuma [now =Antonovo] $\left(47.166^{\circ} \mathrm{N} 142.083^{\circ} \mathrm{E}\right)$, 17.IX.1946, Strelkov (3 フ $\sigma^{7}, 8$ 우, ZISP); Shikotan I., Malokuril'sk $\left(43.869^{\circ} \mathrm{N} 146.829^{\circ} \mathrm{E}\right), 21 . I X .1968$, Gorodkov (2 웅, ZISP); Shikotan I., Tserkovnaya Bay $\left(43.75^{\circ} \mathrm{N} 146.70^{\circ} \mathrm{E}\right)$, 16.VIII.1973, Kerzhner (1 9 , ZISP); same place, 25-30.IX.2012, Yu. Sundukov ( 2 +o, ZMUM); Yankicha I. (47.517 $\left.\mathrm{N} 152.811^{\circ} \mathrm{E}\right)$, 16.VII.1958, Violovich (2 + ㅇ, ZISP); Zelenyy I. $\left(43.51^{\circ} \mathrm{N}\right.$ $\left.146.137^{\circ} \mathrm{E}\right), 2$.VIII.1955, Violovich ( $8 \sigma^{\top} \sigma^{7}, 20$ 우, ZISP). See also Ozerov \& Krivosheina [2014: 217, including S. hadleyi]

DESCRIPTION. Male. Female. Body-length 5.8$9.8 \mathrm{~mm}$.

Thorax, abdomen and legs of most males, especially large exemplares, covered with dense, long, blackish hairs. Females have hairs less dense and shorter.

Head. Frontal vitta reddish-yellow, with delicate whitish reflection; fronto-orbital plate black, greyish dusted. Face, parafacial and gena yellow, with whitish or golden reflection. Postcranium black. 3 orbital and 4-5 frontal setae present. Scapus and pedicel reddishyellow or brownish. Postpedicel black, approximately 2 times as long as wide. Arista nearly bare, longest hairs not exceeding greatest diameter of arista. Palpus yellow.

Thorax black, densely greyish dusted, with dark stripes and fuzzy spots on scutum and scutellum. Acrostichals in two rows, prescutellar pair stronger than the other acrostichals, dorsocentrals $4+4$, intra-alars $1+2$, supra-alars $1+2$, postpronotals 2 . Anepimeron covered with hairs. Scutellum black, greyish dusted, with a pair of strong basal scutellar and a pair of strong apical scutellar setae, also with a pair of discal setulae.

Legs densely greyish dusted. All coxae black; all femora from black to yellow, as a rule fore femur always blackish; all tibiae and tarsi yellow. Male legs covered with long hairs, clearly visible setae on mid and hind tibiae only. Female fore femur also without conspicuous setae. Fore tibia with 2-3 anterodorsal, 2 posterodorsal, 1 posterior, 1 preapical dorsal, and 1 posterior apical setae. Mid femur with a row of anterodorsal setae, also with 1 preapical posterior and 1 preapical posterodorsal setae. Mid tibia with 3 posterodorsal, 2 anterodorsal, 1 ventral, 1 posterior setae and a ring of apicals. Hind femur with a row of anterodorsal setae. Hind tibia with 4-5 anterodorsal, 4-5 posterodorsal, 1 preapical dorsal, 1 preapical anterodorsal, 1 apical anterior, 1 apical anteroventral, and 1 apical ventral setae.

Wing tinged with brownish; crossveins r-m and d-m not darkened.

Abdomen black, densely greyish dusted, covered with dense hairs in male; in female tergites with black marginal setulae. Male sternite 4 about twice as long as wide (Fig. 26); sternite 5 with filiform lateral lobes and a pair of median processes (Fig. 79). Epandrium, cercal plate and surstyli as in Figs 80, 81.

DISTRIBUTION. Russia: Pacific coast. - North America.

\section{Scathophaga karelica Ozerov, 2013}

Figs 27, 82-84, 139.

karelica Ozerov, 2013: 83 (Scathophaga). Type-locality: Poyakonda $\left(66.589^{\circ} \mathrm{N} 32.828^{\circ} \mathrm{E}\right)$ (Russia: Karelia).

REMARK. The species is known from three males from Karelia only [Ozerov, 2013: 83].

DESCRIPTION. Male. Body-length 5.8-6.2 mm.

Head. Frontal vitta reddish-yellow in lower part and blackish around ocellar triangle, with delicate whitish reflection; fronto-orbital plate black, greyish dusted. Face, parafacial and gena reddish-yellow, with whitish reflection. Postcranium black. 3 orbital and 56 frontal setae present. Antenna black. Postpedicel approximately 2 times as long as wide. Arista bare. Palpus yellow.

Thorax black, greyish dusted. Scutum with double brownish line down the middle, and an obscure one on each side. Acrostichals in two rows, dorsocentrals $2+3$, intra-alars $1+2$, supra-alars $1+2$, postpronotals 2 . Anepimeron covered with hairs. Scutellum black, greyish dusted, with a pair of strong basal scutellar and a pair of strong apical scutellar setae. 
Legs. Coxae, trochanters, femora black, greyish dusted; tibiae and tarsi brownish. Fore femur also without conspicuous setae. Fore tibia with 2 dorsal, 2 posterodorsal, 1 posterior, 1 preapical dorsal, and 1 posterior apical setae. Mid femur with 2-5 anterodorsal setae in apical half, also with 1 preapical posterior and 1 preapical posterodorsal setae. Mid tibia with 3 posterodorsal, 2 anterodorsal, 1-2 ventral, 1-2 posterior setae and a ring of apicals. Hind femur with a row of anterodorsal setae. Hind tibia with 3 anterodorsal, 3 posterodorsal, 2-3 anteroventral, 1 preapical dorsal, 1 preapical anterodorsal, 1 apical anterior, 1 apical anteroventral, and 1 apical ventral setae.

Wing tinged with brownish; crossveins r-m and d-m not darkened.

Abdomen black, greyish dusted, covered with not long hairs. Tergites 6-8 and epandrium covered with black setae (Fig. 139). Sternite 4 about 1.5 times as long as wide (Fig. 27); sternite 5 as in Fig. 82. Surstyli very short (Figs 83, 84).

Female unknown.

NOTE. All flies were collected on stones in littoral zone of White Sea.

DISTRIBUTION. Russia: Karelia.

\section{Scathophaga lapponica (Ringdahl, 1920)}

Figs 10-12, 28, 85-87, 133.

lapponica Ringdahl, 1920: 39 (Coniosternum). Type-locality: "Kiruna in Lappland" (Norrbotten, Sweden).

REMARK. This species was recorded in Russia from Kola Peninsula [Gorodkov, 1970: 454], Siberia [Gorodkov, 1986: 32, without specifying the place of collecting], Commander Islands [Gorodkov, 1986: 32] and Amur Oblast [Ozerov, Krivosheina, 2014: 217].

MATERIAL EXAMINED. Altai: Kosh-Agach, plato Ukok, $2400 \mathrm{~m}$, surrounding area of Lake Muzdy-Bulak $\left(49.26^{\circ} \mathrm{N} 87.65^{\circ} \mathrm{E}\right)$, 10.VII.2008, A. Barkalov (1 $0^{7}, 1$, ISEA and ZMUM); Shebalino env. $\left(51.57^{\circ} \mathrm{N} 85.58^{\circ} \mathrm{E}\right)$, floodplain of the River Sema , 462 m, 27-30.VI.2009, V. Sorokina (2 $9+$, ISEA); Amur Oblast: Bol'shoy Never $\left(53.978^{\circ} \mathrm{N} 124.150^{\circ} \mathrm{E}\right)$, 7-8.IX.1974, Gorodkov (14 $\sigma^{7} O^{7}, 8$ 우, ZISP); Arkhangelsk Oblast: Belush'e env $\left(66.89^{\circ} \mathrm{N} 47.61^{\circ} \mathrm{E}\right), 24$. VIII.1978, Gorodkov (1 $\mathrm{O}^{7}$, ZISP); Mezen' $\left(65.85^{\circ} \mathrm{N} 44.24^{\circ} \mathrm{E}\right), 27$. VIII.1978, Gorodkov (1 , Z ZISP); Nizhnyaya Pesha $\left(66.751^{\circ} \mathrm{N} 47.760^{\circ} \mathrm{E}\right), 22$ and 23.VIII.1978, Gorodkov (2 $\bigcirc^{7} \sigma^{7}, 2$ 우, ZISP); Buryatia: Khargun, $6 \mathrm{~km} \mathrm{E}$ of Kyren $\left(51.683^{\circ} \mathrm{N} 102.132^{\circ} \mathrm{E}\right)$, Sayany, 11.VII.1965, Gorodkov (1 9 , ZISP); Mondy env. (51.675 $\left.\mathrm{N} 100.992^{\circ} \mathrm{E}\right)$, 26.VII.1965, Gorodkov (1 $\sigma^{2}$, ZISP); Kamchatka Krai: Commander Islands, Nikol'skoe $\left(55.196^{\circ} \mathrm{N} 165.998^{\circ} \mathrm{E}\right)$, 14.IX.1959, Gorodkov (3 $0^{7} \sigma^{7}$, ZISP); Krasnoyarsk Krai: Khatanga $\left(71.975^{\circ} \mathrm{N} 102.482^{\circ} \mathrm{E}\right), 24$ 26.VIII.1970, V. Zherikhin \& I. Sukacheva (1 $0^{7}$, ZMUM); Khatanga $\left(71.981^{\circ} \mathrm{N} 102.483^{\circ} \mathrm{E}\right), 22-26 . V I I I .1971$, Gorodkov $\left(8 \mathrm{O}^{7} \mathrm{O}^{7}\right.$, 8 우, ZISP); Norilsk $\left(69.339^{\circ} \mathrm{N} 88.220^{\circ} \mathrm{E}\right), 26$.VIII.1975, Gorodkov (1 $0^{7}$, ZISP); Taymyr, Taymyrskiy Nature Reserve, cordon Ary-Mas $\left(72.5^{\circ} \mathrm{N} 101.94^{\circ} \mathrm{E}\right), 14 \mathrm{~m}, 9-22 . \mathrm{VII} .2010$, A. Barkalov (1 $\mathrm{O}^{7}$, ZMUM); Magadan Oblast: Chaybukha $\left(61.801^{\circ} \mathrm{N}\right.$ $\left.160.413^{\circ} \mathrm{E}\right)$, 2.IX.1987, Gorodkov ( $\sigma^{7}, 1$ ㅇ, ZISP); Gizhiga $\left(61.948^{\circ} \mathrm{N} 160.377^{\circ} \mathrm{E}\right)$, 5.IX.1987, Gorodkov (4 O $^{\top} \mathrm{O}^{\top}, 4$ 우, ZISP); Sakhalin Oblast: Paramushir I., Severo-Kuril'sk $\left(50.679^{\circ} \mathrm{N}\right.$ $\left.156.132^{\circ} \mathrm{E}\right)$, 5.IX.1968, Gorodkov (1 $\mathrm{O}^{\top}$, ZISP); Yakutia: Chokurdakh $\left(70.618^{\circ} \mathrm{N} 147.895^{\circ} \mathrm{E}\right), 19-20$. VIII.1971, Gorodkov $\left(6{\bigcirc^{7}}^{7}, 4\right.$ OO, ZISP); Deputatskiy $\left(69.319^{\circ} \mathrm{N} 139.966^{\circ} \mathrm{E}\right), 6$ 7.VIII.1974, Gorodkov (14 O $^{7} 0^{7}, 11$ 우, ZISP); Kular $\left(70.573^{\circ} \mathrm{N}\right.$

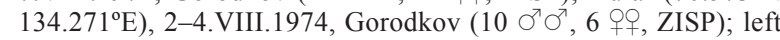
bank of the River Yana opposite of Verkhoyansk $\left(67.550^{\circ} \mathrm{N}\right.$
133.359 ${ }^{\circ}$ E), 23.VII.1974, Nartshuk (1 $\bigcirc^{7}$, ZISP); Mikhaylovka, $60 \mathrm{~km} \mathrm{NE}$ of Amga $\left(61.214^{\circ} \mathrm{N} 132.681^{\circ} \mathrm{E}\right), 12-24$.VIII.1985, 23.VII.1986, Maksimova \& Sivtsov (4 우, ZISP); same place, 20.VII.1986, Bagachanova (1 $\sigma^{7}$, ZISP); Ust'-Kuyga $\left(70.000^{\circ} \mathrm{N}\right.$ 135.562 ${ }^{\circ}$ E), 26-28.VII.1974, Gorodkov (10 $\mathrm{O}^{7} \mathrm{O}^{7}, 8$ 우, ZISP); Zhigansk $\left(66.766^{\circ} \mathrm{N} 123.372^{\circ} \mathrm{E}\right), 15$.VIII.1973, Gorodkov (4 $\mathrm{O}^{7} \mathrm{O}^{\prime \prime}$, 3 우 , ZISP). See also Ozerov \& Krivosheina [2014: 217].

DESCRIPTION. Male. Female. Body-length 6.0$6.8 \mathrm{~mm}$

Head. Frontal vitta yellow or reddish-yellow, with delicate whitish reflection; fronto-orbital plate blackish, densely greyish dusted. Face, parafacial and gena yellow, with whitish reflection. Postcranium blackish. 3 orbital and 4-5 frontal setae present. Antenna black; postpedicel about twice as long as wide. Arista bare. Palpus yellow.

Thorax black, densely greyish dusted, with dark stripes and fuzzy spots on scutum and scutellum. Acrostichals not differentiated from the other hairs on scutum, dorsocentrals $2+3$, intra-alars $1+(1-2)$, supra-alars $1+2$, postpronotals 2 . Anepimeron bare. Scutellum greyish dusted, with a pair of strong basal scutellar and a pair of strong apical scutellar setae.

Legs. All coxae black, greyish dusted. All femora greyish dusted, black, except yellow apex. All tibiae and all tarsi yellow. Fore femur covered with whitish hairs, with 3-4 dorsal setae or setulae in apical third. Fore tibia with 2-4 dorsal, 2-4 posterodorsal, 1-2 posterior, 1 preapical dorsal, 1 preapical anterodorsal, and 1 posterior apical setae. Mid femur with a row of anterior setae, 1 preapical posterior and 1 preapical posterodorsal setae. Mid tibia with 1-2 anterodorsal, 1-2 posterodorsal, 0-1 anteroventral, 0-1 posterior setae, also with a ring of apicals. Hind femur with a row of anterodorsal setae. Hind tibia with $2-3$ posterodorsal, 2-3 anterodorsal, 1 preapical dorsal, 1 preapical anterodorsal and 1 apical anteroventral setae.

Wing clear; crossveins r-m and d-m not darkened.

Abdomen black, densely pale grey dusted, covered with short whitish hairs; tergites 2-6 each with a row of marginal setulae in both sexes. Male sternite 4 about twice as long as wide (Fig. 28). Male sternite 5 with long narrow lateral lobes (Fig. 85). Cercal plate slightly shorter than surstyli (Figs 86, 87). Aedeagus as in Fig.133. End of female abdomen as in Figs 10-12.

DISTRIBUTION. Russia: north of European part, and from northern Siberia east to Magadan Oblast and Kamchatka (Commander Islands), south to Altay and Amur Oblast. - North America; widely distributed, but is not a common species.

\section{Scathophaga litorea (Fallén, 1819)}

Figs 6, 8, 13, 29, 88-90, 138.

litorea Fallén, 1819: 4 (Scatomyza). Type-locality: “ad litus maris balthici" (Sweden).

nigripes Holmgren, 1869: 34 (Scatomyza). Type-locality: "Beeren Eiland... Spetsbergia ad Nordfjorden" (Bear Island and Spitsbergen, Norway).

islandica Becker, 1894: 175 (Scatophaga). Type-locality: "Island"'[= Iceland].

stuxbergii Holmgren, 1880: 24 (Scatomyza). Type-locality: not given [?Russia: Novaya Zemlya]. 
rufiventris Villeneuve, 1917: 308 (Scatophaga). Type-locality: "Gatteville, ... Barfleur, côté normande" (France).

REMARK. The species was recorded in Russia from north of European part (coast of Baltic, Barents, White and Pechora Seas [Gorodkov, 1970: 451, 1986: 32; Engelmark, 1999: 158, 159], Kamchatka [Hendel, 1930: 2, as S.islandica], Pacific coast from Chukotka south to Kuril Islands [Ozerov, Krivosheina, 2014: 218].

MATERIAL EXAMINED. Arkhangelsk Oblast: Kolguev I., Bugrino $\left(68.783^{\circ} \mathrm{N} 49.306^{\circ} \mathrm{E}\right), 21 . \mathrm{VIII} .1970$, Gorodkov $\left(1 \mathrm{O}^{7}, 1\right.$ ㅇ, ZISP); Novaya Zemlya, Krestovaya Bay $\left(74.128^{\circ} \mathrm{N} 55.534^{\circ} \mathrm{E}\right)$, 31.VIII.1921, Nazarov (1 $0^{7}$, ZMUM); Novaya Zemlya, Pukhovaya Bay $\left(72.645^{\circ} \mathrm{N} 52.773^{\circ} \mathrm{E}\right)$, 14.IX.1921, Nazarov (1 $\mathrm{O}^{7}$, ZMUM); Solovetskie Islands, Bol'shoy Solovetsky I. $\left(65.090^{\circ} \mathrm{N} 35.639^{\circ} \mathrm{E}\right)$, 17.VIII.1963 (1 $\sigma^{7}$, ZISP); Chukotka: $5 \mathrm{~km} \mathrm{~N}$ of Egvekinot $\left(66.395^{\circ} \mathrm{N} 179.132^{\circ} \mathrm{W}\right), 26 . V I I .1963$, Gorodkov (12 $0^{7} \sigma^{7}$, ZISP); Meynypil'gyno env. $\left(62.555^{\circ} \mathrm{N} 177.063^{\circ} \mathrm{E}\right), 29 . \mathrm{VII} .2015$, P.S. Tomkovich (1 $\sigma^{7}$, ZMUM); Provideniya Bay $\left(64.412^{\circ} \mathrm{N} 173.307^{\circ} \mathrm{W}\right)$, 14.IX.1911, Starokad (1 $0^{7}$, ZISP); Kamchatka Krai: Commander Islands, Mednyy I. (ca $54.790^{\circ} \mathrm{N} 167.578^{\circ} \mathrm{E}$ ), 1.VII.1958, Violovich (1 $\sigma^{7}$, ZISP); Commander Islands, Nikol'skoe $\left(55.196^{\circ} \mathrm{N}\right.$ 165.998 ${ }^{\circ}$ E), 9.IX.1959, Gorodkov (4 $\mathrm{O}^{7} \mathrm{O}^{7}$, ZISP); Korf $\left(60.376^{\circ} \mathrm{N}\right.$ $\left.166.023^{\circ} \mathrm{E}\right)$, 9.IX.1973, Negrobov (1 $\mathrm{O}^{7}$, ZISP); PetropavlovskKamchatskiy $\left(53.013^{\circ} \mathrm{N} 158.657^{\circ} \mathrm{E}\right), 30-31 . V I I I .1969$, Gorodkov $\left(14 \bigcirc^{7} \odot^{7}, 6\right.$ + 9 , ZISP); the mouth of the River Kygchik (ca. $53.411^{\circ} \mathrm{N}$ $\left.156.742^{\circ} \mathrm{E}\right)$, 18.VIII.1911, Soldatov $\left(2 \sigma^{7} \sigma^{7}, 1\right.$ q, ZISP); Ust'Kamchatsk $\left(56.229^{\circ} \mathrm{N} 162.469^{\circ} \mathrm{E}\right), 10 . \mathrm{VIII} .1930$, E. Gur'yanova (1 $\mathrm{O}^{7}$, ZISP); Karelia: Kartesh $\left(66.33^{\circ} \mathrm{N} 33.64^{\circ} \mathrm{E}\right), 18$ and 22.VII.1975, Gorodkov ( $10^{7}, 1$ ․, ZISP); Keret' I. $\left(66.313^{\circ} \mathrm{N} 33.719^{\circ} \mathrm{E}\right)$, 8.IX.1984, Gorodkov (3 $\sigma^{7} \mathrm{O}^{7}$, ZISP); Poyakonda $\left(66.589^{\circ} \mathrm{N}\right.$ $\left.32.828^{\circ} \mathrm{E}\right)$, 30.VI. and 7.VII.2010, A.L. Ozerov $\left(28 \mathrm{O}^{7} \mathrm{O}^{7}, 16\right.$ 우, ZMUM); Primorsky env. $\left(66.549^{\circ} \mathrm{N} 33.133^{\circ} \mathrm{E}, 66.536^{\circ} \mathrm{N} 33.087^{\circ} \mathrm{E}\right.$, $\left.66.552^{\circ} \mathrm{N} 33.100^{\circ} \mathrm{E}\right), 1-9$. VII.2010, A.L. Ozerov $\left(18 \mathrm{O}^{7} 0^{7}, 9\right.$ 우, ZMUM); Ryazhkov I. $\left(67.017^{\circ} \mathrm{N} 32.556^{\circ} \mathrm{E}\right), 12 . \mathrm{VI} .1992$, E.V. Shutova (2 $\left.\sigma^{7} O^{7}, Z M U M\right)$; Voron'ya Guba $\left(66.937^{\circ} 9 \mathrm{~N} 32.472^{\circ} \mathrm{E}\right)$, 21.VIII.1982, Erlykova (1 $\bigcirc^{7}$, ZISP); Khabarovsk Krai: Ochotsk env. $\left(59.366^{\circ} \mathrm{N} 143.229^{\circ} \mathrm{E}\right), 25$. VIII.1987, Gorodkov (13 $\mathrm{O}^{7} \sigma^{7}, 3$ 우, ZISP); Magadan Oblast: Evensk $\left(61.916^{\circ} \mathrm{N} 159.234^{\circ} \mathrm{E}\right)$, 8.IX.1987, Gorodkov (17 $\sigma^{7} \sigma^{7}$, ZISP); Gizhiga $\left(61.948^{\circ} \mathrm{N}\right.$ $\left.160.377^{\circ} \mathrm{E}\right), 5$ and 6.IX.1987, Gorodkov (1 $0^{7}, 1$ + , ZISP); Koni Peninsula, cordon "Mys Ploskiy" $\left(59.15^{\circ} \mathrm{N} 151.63^{\circ} \mathrm{E}\right), 30$.VI. and 4-7.VII.2017, Sorokina (3 $\sigma^{7} \sigma^{7}$, ZISP); Koni Peninsula, Cape Plosky $\left(59.161^{\circ} \mathrm{N} 151.643^{\circ} \mathrm{E}\right), 16$. VII.2015 and 3.VII.2016, N. Tridrikh ( 8 $\mathrm{O}^{7} \sigma^{7}, 1$ + , ZMUM); Murmansk Oblast: Aleksandrovsk [= Polyarnyy] $\left(69.198^{\circ} \mathrm{N} 33.456^{\circ} \mathrm{E}\right), 12 . \mathrm{VI} .1921$, Zhelokhovtsev (1 $\mathrm{O}^{7}$, ZMUM); same place, 17.IX.1928, Cheburova (39 $0^{7} 0^{7}, 21$ 우, ZMUM); Dal'nie Zelentsy $\left(69.117^{\circ} \mathrm{N} 36.062^{\circ} \mathrm{E}\right), 6$ and 9.VIII.1981, Gorodkov (4 $0^{7} 0^{7}, 1$ ㅇ, ZISP); Kashkarantsy $\left(66.340^{\circ} \mathrm{N} 36.031^{\circ} \mathrm{E}\right)$, 15.VIII.1995, Gorodkov (1 $\sigma^{2}$, ZISP); Murmansk $\left(68.918^{\circ} \mathrm{N}\right.$ 33.059 ${ }^{\circ}$ ) , 9-13.VIII.2010, N. Vikhrev (4 $0^{7} \sigma^{7}$, ZMUM); Olenitsa $\left(66.471^{\circ} \mathrm{N} 35.348^{\circ} \mathrm{E}\right)$, 15.VIII.1995, Gorodkov (1 $\mathrm{O}^{7}$, ZISP); Por'ya Bay $\left(66.764^{\circ} \mathrm{N} 33.669^{\circ} \mathrm{E}\right), 12 . V I .1992$, E.V. Shutova $\left(3 \mathrm{O}^{7} \mathrm{O}^{7}, 1\right.$, ZMUM); River Kola $\left(68.886^{\circ} \mathrm{N} 33.0236^{\circ} \mathrm{E}\right)$, 19.VII.2011, A. Ozerov, D. Gavryushin $\left(8 \mathrm{O}^{7} \mathrm{O}^{7}, 1\right.$ ㅇ, ZMUM); Sosnovka $\left(66.507^{\circ} \mathrm{N}\right.$ $\left.40.583^{\circ} \mathrm{E}\right), 23$.VIII.1981, Gorodkov (2 $\mathrm{O}^{7} \mathrm{O}^{7}, 1$, , ZISP); Teriberka $\left(69.164^{\circ} \mathrm{N} 35.140^{\circ} \mathrm{E}\right)$, 3.VII.2008, V. Semenov (3 $0^{7} 0^{7}$, ZMUM); Voroniy I. (66.994 $\left.\mathrm{N} 33.666^{\circ} \mathrm{E}\right), 24$. VI.1992, E.V. Shutova $\left(2 \mathrm{O}^{\mathrm{T}} \mathrm{O}^{7}\right.$, 1 , ZMUM); Kol'sky Bay $\left(68.951^{\circ} \mathrm{N} 33.033^{\circ} \mathrm{E}\right), 3$ and 18.VII.1924, Kapustin (5 $0^{7} \sigma^{7}, 2$ ++ , ZISP); Sakhalin Oblast: Kunashir I., Golovnino $\left(43.737^{\circ} \mathrm{N} 145.517^{\circ} \mathrm{E}\right), 27-28 . I X .1968$, Gorodkov (29 $0^{7} O^{7}, 21$ OQ, ZISP); Paramushir I., Severo-Kuril'sk $\left(50.679^{\circ} \mathrm{N}\right.$ $\left.156.132^{\circ} \mathrm{E}\right)$, 9.IX.1968, Gorodkov (3 $\sigma^{7} \sigma^{7}, 3$ 우, ZISP). See also Ozerov \& Krivosheina [2014: 218]. $6.8 \mathrm{~mm}$.

DESCRIPTION. Male. Female. Body-length 4.3-

Head. Frontal vitta reddish-yellow completely or reddish-yellow in lower half and blackish around ocellar triangle, with delicate whitish reflection; frontoorbital plate black, greyish dusted. Face, parafacial and gena reddish-yellow, with whitish or golden reflection.
Postcranium black. 3 orbital and 5-6 frontal setae present. Antenna black. Postpedicel approximately 2 times as long as wide. Arista bare. Palpus yellow.

Thorax black, greyish dusted. Scutum with double brownish line down the middle, and an obscure one on each side. Acrostichals in two rows, dorsocentrals $2+3$, intra-alars $1+2$, supra-alars $1+2$, postpronotals 2 . Anepimeron covered with hairs. Scutellum black, greyish dusted, with a pair of strong basal scutellar and a pair of strong apical scutellar setae.

Legs greyish dusted. Coxae and trochanters black; femora from yellow to black, as a rule black; tibiae and tarsi brownish, sometimes fore and hind tibiae blackish. Fore femur covered with more or less long whitish and blackish hairs, but without conspicuous setae. Fore tibia with 2 dorsal, 2 posterodorsal, 1 posterior, 1 preapical dorsal, and 1 posterior apical setae. Mid femur usually with 2-5 anterodorsal setae in apical half in male and a row of anterodorsal setae in female, also with 1 preapical posterior and 1 preapical posterodorsal setae in both sexes. Mid tibia with 3 posterodorsal, 2 anterodorsal, 1-2 ventral, 1-2 posterior setae and a ring of apicals; ventral and posterior setae strong in female and hair-like in male. Hind femur without conspicuous setae in male and with a row of anterodorsal setae in female. Hind tibia with 3 anterodorsal, 3 posterodorsal, 2-3 anteroventral, 1 preapical dorsal, 1 preapical anterodorsal, 1 apical anterior, 1 apical anteroventral and 1 apical ventral setae.

Wing tinged with brownish; crossveins r-m and d-m not darkened (Fig. 8).

Abdomen black, greyish dusted, covered with not long hairs, female tergiters 2-6 each with a row of marginal setae. Male end of abdomen as in Fig. 138. Male sternite 4 about 2 times as long as wide (Fig. 29). Male sternite 5, epandrium, cercal plate and surstyli as in Figs. 88-90.

DISTRIBUTION. Russia: sea coast of north European part (Baltic, Barents, White and Pechora Seas), Pacific coast from Chukotka south to Kuril Islands. Europe (widespread on north Atlantic coast), ?Greenland; a littoral species.

\section{Scathophaga lutaria (Fabricius, 1794)} Figs 30, 91-93.

lutaria Fabricius, 1794: 344 (Musca). Type-locality: "Habitat Kiliae" [= Kiel, Germany].

maculipes Zetterstedt, 1846: 1964 (Scatomyza). Type-localities: "Jemtlandia... Lapponia Umensi... Norwegia ad diversorium Suulstuen" (Sweden, Norway).

parviceps Ringdahl, 1936: 175 (Scopeuma). Type-locality: "Vällista-Gebirge in Jämtland" (Sweden).

REMARK. This species was registered in Russia from Kola Peninsula and Crimea [Gorodkov, 1970: 451], Siberia (Dudunka) [Ozerov, Barkalov, 2014: 563], Astrakhan' Oblast [Ovchinnikov, 2004: 422], Yakutia [Sychevskaya, 1972: 150; Veselkin, 1985: 75; Bagachanova et al. 2016: 782].

MATERIAL EXAMINED. Altai: Biysk $\left(52.535^{\circ} \mathrm{N} 85.178^{\circ} \mathrm{E}\right)$,

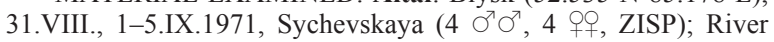


Chuya, $20 \mathrm{~km} \mathrm{NW}$ of Kosh-Agach $\left(50.085^{\circ} \mathrm{N} 88.404^{\circ} \mathrm{E}\right), 25$ 27.VII.2006, Nartshuk (1 $\sigma^{7}$, ZISP); Arkhangelsk Oblast: Solovetskiy I. $\left(65.080^{\circ} \mathrm{N} 35.686^{\circ} \mathrm{E}\right), 26$.VII.1959, Gorodkov (1 $\sigma^{7}$, ZISP); Solvychegovsk $\left(61.336^{\circ} \mathrm{N} 46.900^{\circ} \mathrm{E}\right), 15$.VIII.2010, D. Gavryushin $\left(1 \sigma^{7}\right.$, ZMUM); Talagi $\left(64.620^{\circ} \mathrm{N} 40.648^{\circ} \mathrm{E}\right), 11$.VIII.1970, Gorodkov (2 $\sigma^{7} \sigma^{7}$, ZISP); Astrakhan Oblast: Baskunchak salt Lake $\left(48.193^{\circ} \mathrm{N} 46.813^{\circ} \mathrm{E}\right), 2-4 . V .2010, \mathrm{~K}$. Tomkovich (1 $\left.\sigma^{7}, \mathrm{ZMUM}\right)$; Ikryanoe $\left(46.112^{\circ} \mathrm{N} 47.767^{\circ} \mathrm{E}\right), 10 . \mathrm{V} .2010, \mathrm{~K}$. Tomkovich $\left(2 \bigcirc^{7} \sigma^{7}\right.$ 1 , ZMUM); Bashkiria: Bashkirskiy Nature Reserve $\left(53.344^{\circ} \mathrm{N}\right.$ 57.783ㅌ), 7.VIII.1975, Gorodkov (1 +, ZISP); between Abzakovo and Murakaevo (ca. $53.797^{\circ} \mathrm{N} 58.651^{\circ} \mathrm{E}$ ), 2-8.VIII.2008, K. Tomkovich (1 $\mathrm{O}, \mathrm{ZMUM})$; Ufa env. $\left(54.776^{\circ} \mathrm{N} 56.068^{\circ} \mathrm{E}\right), 18$.VIII.1975, Gorodkov (1 $\sigma^{\top}, 2$ 90 , ZISP); Buryatia: Arshan $\left(51.908^{\circ} \mathrm{N}\right.$ $\left.102.428^{\circ} \mathrm{E}\right)$, 2.VII.1965, Negrobov (1 $\sigma^{7}$, ZISP); Mondy env. $\left(51.675^{\circ} \mathrm{N} 100.992^{\circ} \mathrm{E}\right), 1900 \mathrm{~m}, 21 . \mathrm{VII} .1962$, Gorodkov (1 $\mathrm{O}^{7}$, ZISP); same place, 30.VII.1965, A. Pleshanov (1 ㅇ, ZISP); Chelyabinsk Oblast: Katav-Ivanovsk $\left(54.751^{\circ} \mathrm{N} 58.197^{\circ} \mathrm{E}\right), 2 . \mathrm{VII} .19269$, Vakulenko ( 1 + , ZISP); Taganay $\left(55.277^{\circ} \mathrm{N} 59.795^{\circ} \mathrm{E}\right), 18$ 24.VII.2008, K. Tomkovich (2 $\bigcirc^{7} \sigma^{7}$, ZMUM); Crimea: Alushta $\left(44.677^{\circ} \mathrm{N} 34.403^{\circ} \mathrm{E}\right), 19 . V .1900, \mathrm{~N}$. Kuznetsov (1 $\left.\sigma^{7}, \mathrm{ZISP}\right)$; Chatyr-Dag $\left(44.862^{\circ} \mathrm{N} 34.224^{\circ} \mathrm{E}\right), 25 . \mathrm{V} .1963$, Gorodkov (1 ㅇ, ZISP); Chufut-Kale $\left(44.741^{\circ} \mathrm{N} 33.922^{\circ} \mathrm{E}\right), 24$. VIII.1971, Kasparyan (1 $\sigma^{\prime}$, ZISP); south coast (ca. $44.483^{\circ} \mathrm{N} 33.866^{\circ} \mathrm{E}$ ), 1901, Yu. Vagner (1 $\sigma^{7}, 1$, ZISP); Kaliningrad Oblast: Kurshskaya Kosa $\left(55.154^{\circ} \mathrm{N}\right.$ $20.857^{\circ} \mathrm{E}$ ), without data, V. Kolyda (1 $\sigma^{7}$, ZMUM); Rybachiy $\left(55.157^{\circ} \mathrm{N} 20.841^{\circ} \mathrm{E}\right), 12 . I X .1972$, Gorodkov (1 $\mathrm{O}^{7}$, ZISP); Kalmykia: Tsagan Aman $\left(47.562^{\circ} \mathrm{N} 46.717^{\circ} \mathrm{E}\right), 14$. VI.1979, Emel'yanenko (1 $\sigma^{7}$, ZISP); Karelia: Kartesh $\left(66.33^{\circ} \mathrm{N} 33.64^{\circ} \mathrm{E}\right)$, 20.VII.1975, Gorodkov (1 $\sigma^{7}$, ZISP); Komi: $3 \mathrm{~km} \mathrm{~N}$ of Ust-Tsilma (ca. $65.45^{\circ} \mathrm{N}$ $\left.52.11^{\circ} \mathrm{E}\right)$, 10.VIII.1978, Gorodkov (1 $\sigma^{7}, 1$ ㅇ, ZISP); TroitskoPechersk $\left(62.712^{\circ} \mathrm{N} 56.184^{\circ} \mathrm{E}\right), 11$. VIII.1989, Gorodkov (1 O $\left.^{7} \mathrm{ZISP}\right)$; Ukhta, 3.VIII.1976, Gorodkov (1 $\sigma^{7}, 1$ ㅇ, ZISP); Vil'gort $\left(61.620^{\circ} \mathrm{N}\right.$ $\left.50.752^{\circ} \mathrm{E}\right), 26$.VIII.1957, Gabova (1 ${ }^{7}$, ZISP); Yaksha $\left(61.823^{\circ} \mathrm{N}\right.$ $\left.56.821^{\circ} \mathrm{E}\right)$, 15.VIII.1989, Gorodkov (1 ${ }^{\circ}$, ZISP); Krasnodar Krai Tuapse $\left(44.104^{\circ} \mathrm{N} 39.068^{\circ} 1 \mathrm{E}\right), 26 . X .1913$, Shaposhnikov (4 $\sigma^{\top} \sigma^{\top}, 1$ +, ZISP); Agurskoe gorge $\left(43.548^{\circ} \mathrm{N} 39.814^{\circ} \mathrm{E}\right), 22 . \mathrm{IV} .2008$, A.L.

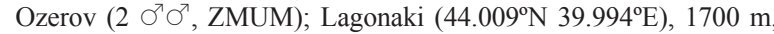
11.VI.2012, N. Vikhrev (1 $\sigma^{7}$, ZMUM); Sochi, Mt Akhun $\left(43.523^{\circ} \mathrm{N}\right.$ $\left.39.879^{\circ} \mathrm{E}\right), 22 . I V .2008$, N. Vikhrev (1 $\sigma^{\top}$, ZMUM); Teberdinskiy Nature Reserve $\left(43.405^{\circ} \mathrm{N} 41.721^{\circ} \mathrm{E}\right), 15 . \mathrm{V} .1964$, Gur'yanova (1 $\sigma^{\prime}$ ZISP); same place, 18.VI.1968, Gorodkov (1 O', ZISP); Krasnoyarsk Krai: Baykit $\left(61.68^{\circ} \mathrm{N} 96.38^{\circ} \mathrm{E}\right)$, 26.VIII.1972, Gorodkov (1

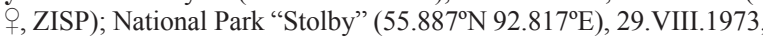
Gorodkov ( $2 \sigma^{7} \sigma^{7}, 2$, $\bigcirc$, ZISP); Kursk Oblast: Oboyan' $\left(51.191^{\circ} \mathrm{N}\right.$ $\left.36.312^{\circ} \mathrm{E}\right), 26 . V .2007$, N. Vikhrev, A.L. Ozerov (1 O', 1 ㅇ, ZMUM); Ryl'sk (51.569 N $34.682^{\circ}$ E), 3.V.1915, Sokolov (1 ㅇ, ZISP); Streletskaya steppe $\left(51.579^{\circ} \mathrm{N} 36.087^{\circ} \mathrm{E}\right), 6 . I X .2007,11 . V I I I .2008, \mathrm{~N}$. Vikhrev $\left(1 \sigma^{7}, 1\right.$,, ZMUM); Leningrad Oblast: Agalatovo $\left(60.217^{\circ} \mathrm{N}\right.$ $\left.30.303^{\circ} \mathrm{E}\right)$, 1.X.1961, A. Stackelberg (1 $\sigma^{\top}$, ZISP); Komarovo $\left(60.188^{\circ} \mathrm{N} 29.812^{\circ} \mathrm{E}\right)$, 2.IX.1950, A. Stackelberg (1 $\sigma^{7}$, ZISP); Luga (ca. $\left.58.73^{\circ} \mathrm{N} 29.84^{\circ} \mathrm{E}\right), 2$. VIII.1955, A. Stackelberg (1 O', ZISP); Rakovichi $\left(58.650^{\circ} \mathrm{N} 29.836^{\circ} \mathrm{E}\right), 10$ and 17.V., 12.VI.,1 and 5.IX.1897, G. Pleske (20 $\sigma^{\top} \sigma^{7}, 5$ 우, ZISP); Saint Petersburg $\left(59.934^{\circ} \mathrm{N} 30.359^{\circ} \mathrm{E}\right), 5 . X .1954$, A. Stackelberg (1 O', ZISP); same place, 17.VIII.1997, Gorodkov (1 $\sigma^{7}$, ZISP); same place, 23.IX.2006, Krivokhatskiy (1 $\sigma^{7}$, ZISP); Yashchera $\left(58.894^{\circ} \mathrm{N} 29.820^{\circ} \mathrm{E}\right)$ 25.VIII.1958, A. Stackelberg (1 $\sigma^{\top}$, ZISP); Moscow and Moscow Oblast: $35 \mathrm{~km} \mathrm{NNE}$ of Moscow $\left(56.200^{\circ} \mathrm{N} 37.833^{\circ} \mathrm{E}\right), 26 . \mathrm{VI} .1993$, A.L. Ozerov (1 $\sigma^{7}$, ZMUM); Abramtsevo $\left(56.230^{\circ} \mathrm{N} 37.956^{\circ} \mathrm{E}\right)$, 15.VI.1958, E.S. Smirnov (1 9 , ZMUM); Bittsa $\left(55.641^{\circ} \mathrm{N} 37.570^{\circ} \mathrm{E}\right)$, 17.VI., 18-31.VII.1936, B. Rohdendorf (3 $\sigma^{7} \sigma^{7}, 4$ oᄋ, ZMUM);

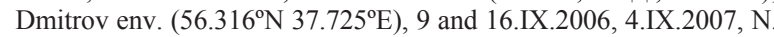
Vikhrev (3 $\sigma^{\top} \sigma^{7}$, ZMUM); Golitsyno $\left(55.649^{\circ} \mathrm{N} 37.011^{\circ} \mathrm{E}\right)$, 3.VII.1977, 31.V. and 4-7.IX.1981, A. Shatalkin (8 $\sigma^{7} \sigma^{7}, 2$ oo, ZMUM); Izmaylovo (55.786 $\left.\mathrm{N} 37.835^{\circ} \mathrm{E}\right)$, 9.IX.1989, 3.VI.1990, A.L. Ozerov (1 $\sigma^{7} \sigma^{7}, 2$ 우, ZMUM); Kosino $\left(55.721^{\circ} \mathrm{N} 37.847^{\circ} \mathrm{E}\right)$, 26.VI.1921 B. Dodonov (1 +, ZMUM); Kuntsevo $\left(55.720^{\circ} \mathrm{N}\right.$ $\left.37.473^{\circ} \mathrm{E}\right), 5 . I X .1981,5 . V I .1982$, A.L. Ozerov ( 3 O $^{7} \sigma^{7}, 3$ 우, ZMUM); Mamontovka $\left(55.999^{\circ} \mathrm{N} 37.816^{\circ} \mathrm{E}\right), 3 . V I I .1917,26 . V I .1956$, E. Smirnov (2 $\sigma^{7} \sigma^{7}, 3$ 우, ZMUM); Naro-Fominsk $\left(55.455^{\circ} \mathrm{N} 36.882^{\circ} \mathrm{E}\right)$, 12.V.2006, D. Gavryushin (1 $\sigma^{7}$, ZMUM); Petrovsko-Razumovskoe $\left(55.839^{\circ} \mathrm{N} 37.569^{\circ} \mathrm{E}\right), 10 . \mathrm{VI} .1931$, E. Smirnov (1 O', ZMUM); Shchukino $\left(55.798^{\circ} \mathrm{N} 37.478^{\circ} \mathrm{E}\right), 19 . I X .2006$, N. Vikhrev (2 O $\sigma^{\top}$, ZMUM);
Smolevo $\left(55.578^{\circ} \mathrm{N} 38.666^{\circ} \mathrm{E}\right), 25-31 . V .2012$, K. Tomkovich (1 ○',

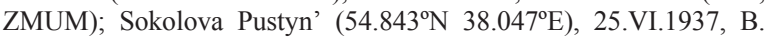
Rohdendorf (1 $\sigma^{7}$, ZMUM); Zelenograd $\left(55.986^{\circ} \mathrm{N} 37.202^{\circ} \mathrm{E}\right)$, 19.VI.1998, A. Gusakov (1 ${ }^{7}, 1$ o, ZMUM); Zvenigorod env. $\left(55.700^{\circ} \mathrm{N} 36.722^{\circ} \mathrm{E}\right), 6$.VII.1954, L. Zimina (1 +, ZMUM); Murmansk Oblast: $8 \mathrm{~km} \mathrm{~N}$ of Revda $\left(68.003^{\circ} \mathrm{N} 34.570^{\circ} \mathrm{E}\right), 15 . \mathrm{VIII} .1981$, Gorodkov (1 $\sigma^{7}$, ZISP); Aleksandrovsk [= Polyarnyy] $\left(69.198^{\circ} \mathrm{N}\right.$ $\left.33.456^{\circ} \mathrm{E}\right), 25$. VIII. and 6.IX.1923, V. Kuznetsov $\left(4 \sigma^{7} \sigma^{\top}, 1\right.$, ZISP); same place, 24.VIII. and 7.IX.1923, Fridolin (2 $\sigma^{\top} \sigma^{\top}, 1$, ZISP); same place, 16.IX.1928, Cheburova (1 ㅇ, ZISP); Dal'nie Zelentsy $\left(69.117^{\circ} \mathrm{N} 36.062^{\circ} \mathrm{E}\right), 26 . V I I I .1984$, Gorodkov (1 $\left.\mathrm{O}^{\top}, \mathrm{ZISP}\right)$; Khibiny Station $\left(67.6736^{\circ} \mathrm{N} 33.2126^{\circ} \mathrm{E}\right), 15,16$ and 19.VIII., 4.IX.1928, Cheburova (1 $0^{7}, 3$ 우, ZISP); Kirovsk env. $\left(67.608^{\circ} \mathrm{N}\right.$ $\left.33.661^{\circ} \mathrm{E}\right)$, 9.VII.1975, Zinov'ev (2 90 , ZISP); Lake Vudyavr basin (ca. $67.646^{\circ} \mathrm{N} 33.644^{\circ} \mathrm{E}$ ), 27 and 28.VIII., 9, 13 and 14.IX.1930, Cheburova (3 $\sigma^{7} \sigma^{7}, 2$ + ,, ZISP); same place, 10 and 30.VII.1931, 12 and 18.IX. 1936, Fridolin (3 ○ ఠ 2 O9, ZISP); same place, 21.VIII.1931, E. Belyakova (1 $\sigma^{\top}, 4$ 우, ZISP); Murmansk $\left(68.918^{\circ} \mathrm{N}\right.$ 33.059 $\left.{ }^{\circ} \mathrm{E}\right)$, 9-13.VIII.2010, N. Vikhrev (1 今, ZMUM); Murmansk env. $\left(68.964^{\circ} \mathrm{N} 33.085^{\circ} \mathrm{E}\right), 14$ and 15.VIII., 28.IX.1923, Fridolin (3

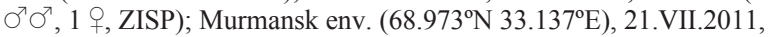
A. Ozerov (1 $\sigma^{\prime}$, ZMUM); River Tuloma $\left(68.738^{\circ} \mathrm{N} 32.288^{\circ} \mathrm{E}\right), 14$ 15.VII.1906, Soldatov ( 1 ㅇ, ZISP); Tumannyy $\left(68.883^{\circ} \mathrm{N} 35.693^{\circ} \mathrm{E}\right)$, 4.VIII.1981, Gorodkov (1 $\sigma^{7}$, ZISP); Novgorod Oblast: Malaya Vishera $\left(58.849^{\circ} \mathrm{N} 32.209^{\circ} \mathrm{E}\right), 9 . V .1975$, Gorodkov (1 $\sigma^{7}$, ZISP); Vereb'e $\left(58.678^{\circ} \mathrm{N} 32.696^{\circ} \mathrm{E}\right), 10 . \mathrm{V} .1975$, Gorodkov (1 + , ZISP); Perm' Oblast: Kungur $\left(57.424^{\circ} \mathrm{N} 56.953^{\circ} \mathrm{E}\right), 29 . \mathrm{VII} .1997$, Gorodkov $\left(1 \sigma^{\top}, 1\right.$ ㅇ, ZISP); Pskov Oblast: Khtiny $\left(58.711^{\circ} \mathrm{N}, 28.597^{\circ} \mathrm{E}\right)$, 31.V.1896, Pleske ( 1 , , ZISP); Rostov Oblast: Kamensk-Shakhtinsky $\left(48.293^{\circ} \mathrm{N} 40.257^{\circ} \mathrm{E}\right), 25$. V.2011, D. Gavryushin (1 $\left.\Im^{7}, \mathrm{ZMUM}\right)$; Smolensk Oblast: "Smolenskoe poozer'e" National Park $\left(55.511^{\circ} \mathrm{N}\right.$ $\left.31.839^{\circ} \mathrm{E}\right), 30$.VII.1991, Zlobin (1 + , ZISP); Tver' Oblast: Bologoe $\left(57.879^{\circ} \mathrm{N} 33.997^{\circ} \mathrm{E}\right), 21 . \mathrm{V} .1902$, F. Zaytseva (1 9 , ZISP); Tyumen' Oblast: Labytnangi $\left(66.657^{\circ} \mathrm{N} 66.391^{\circ} \mathrm{E}\right), 23$.VIII.1973, V. Sychevskaya ( 1 \%, ZMUM); Novyy Urengoy $\left(66.087^{\circ} \mathrm{N} 76.513^{\circ} \mathrm{E}\right)$, 30.VII.1982, Gorodkov (5 $\bigcirc^{7}$, ZISP); Salekhard $\left(66.53^{\circ} \mathrm{N}\right.$ $\left.66.6135^{\circ} \mathrm{E}\right), 4$ and 5.VIII.1976, Gorodkov (2 90, ZISP); Sob' env.

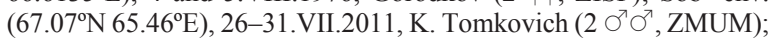
Surgut $\left(61.260^{\circ} \mathrm{N} 73.401^{\circ} \mathrm{E}\right), 27$. VII.1977, Gorodkov (1 $\sigma^{7}$, ZISP); Tyumen' env. $\left(57.15^{\circ} \mathrm{N} 65.53^{\circ} \mathrm{E}\right)$, 5.IX.1976, Gorodkov (1 ㅇ, ZISP); Tarko-Sale $\left(64.914^{\circ} \mathrm{N} 77.766^{\circ} \mathrm{E}\right), 3 . I X .1982$, Gorodkov $\left(1 \mathrm{O}^{7}, 1\right.$, ZISP); Yakutia: Khaptagay $\left(61.785^{\circ} \mathrm{N} 129.797^{\circ} \mathrm{E}\right), 23 . \mathrm{VI} .1974, \mathrm{~L}$. Zimina (1 +, ZMUM); Lensk $\left(60.727^{\circ} \mathrm{N} 114.943^{\circ} \mathrm{E}\right)$, 14.IX.1987, Gorodkov (1 $\sigma^{7}$, ZISP); Yaroslavl' Oblast: Rostov $\left(57.190^{\circ} \mathrm{N}\right.$ $\left.39.414^{\circ} \mathrm{E}\right), \mathrm{VI} .-\mathrm{VII} .1920$, Martynov (1 + , ZISP). See also Ozerov \& Barkalov [2014: 563].

DESCRIPTION. Male. Female. Body-length 3.8$9.2 \mathrm{~mm}$

Head. Frontal vitta yellow or reddish-yellow, matt; fronto-orbital plate blackish, greyish dusted. Face, parafacial and gena yellow, with delicate golden reflection. Postcranium black in upper third or half and yellow in lower part. 3 orbital and 4-6 frontal setae present. Antenna reddish-yellow. Postpedicel approximately twice as long as wide. Arista pubescent, the longest hairs approximately equal to $1 / 2$ width of postpedicel. Palpus yellow.

Thorax black in ground colour, greyish dusted, but usually postpronotal lobe yellowish. Acrostichals not differentiated from the other hairs on scutum or setulose in two rows, only prescutellar pair usually stronger than the other acrostichals, dorsocentrals $2+3$, intraalars $1+2$, supra-alars $1+2$, postpronotals 2 . Katepisternum posteriorly, besides yellow hairs and setulae, as a rule also with several black setulae. Anepimeron bare. Scutellum black, greyish dusted, with a pair of strong basal scutellar and a pair of strong apical scutellar setae. 


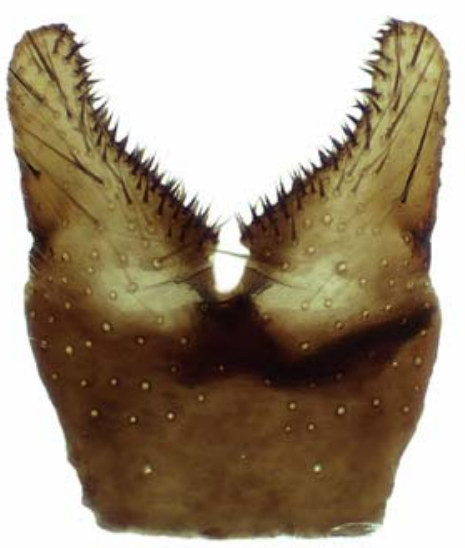

88

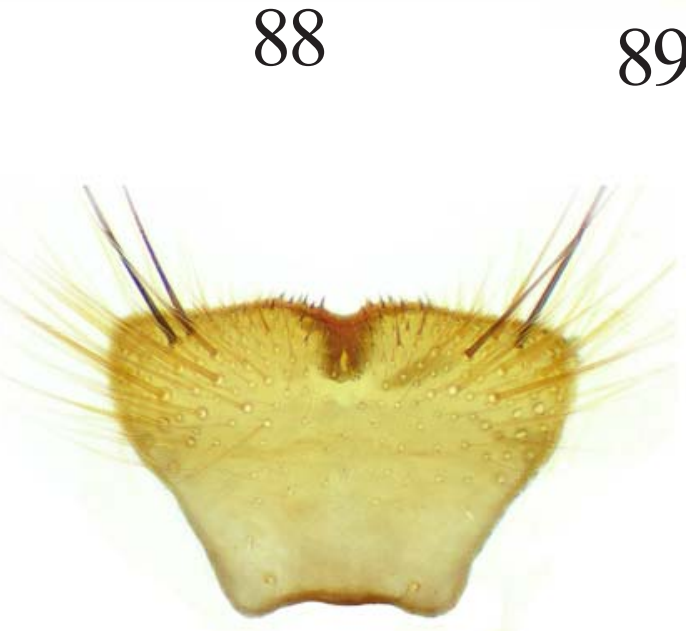

91

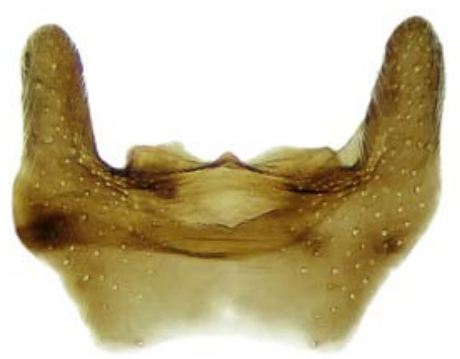

94
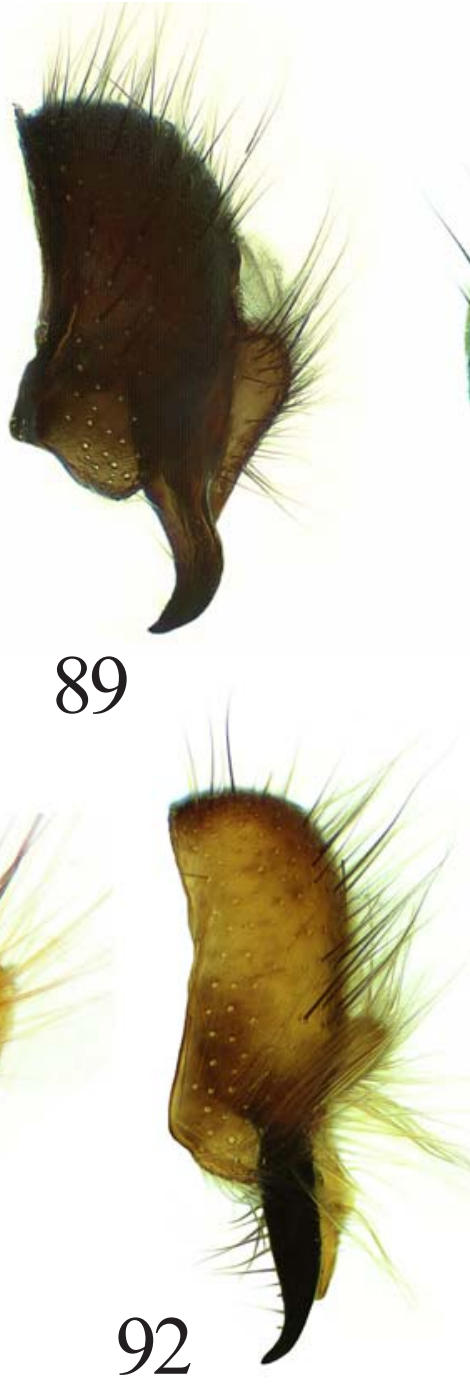

92

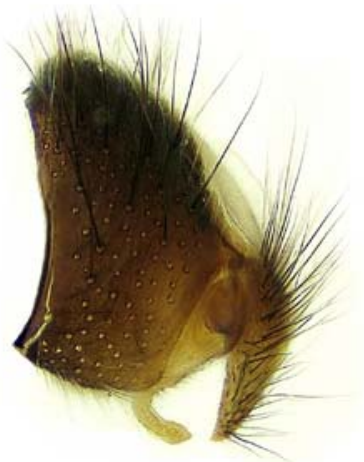

95

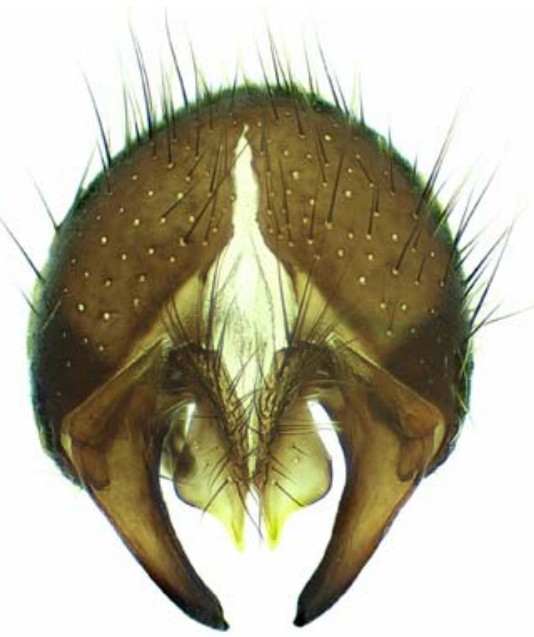

90

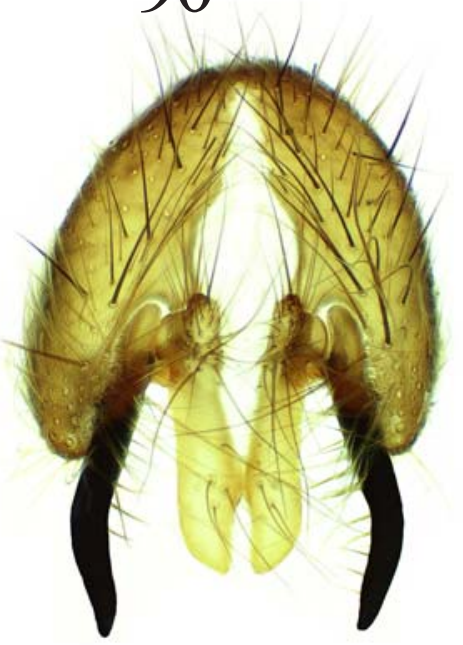

93

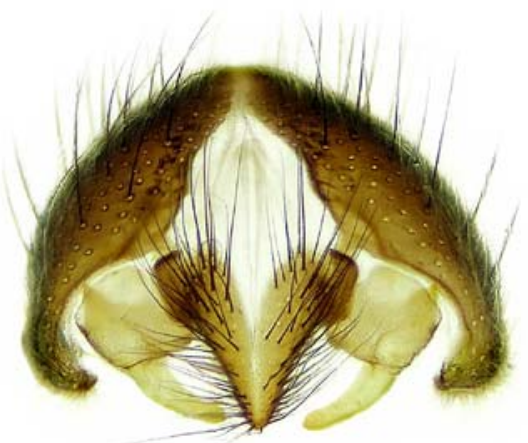

96

Figs 88-96. Scathophaga litorea (Fallén) (88-90), S. lutaria (Fabricius) (91-93) and S. mollis (Becker) (94-96): 88, 91, 94 - male sternite 5; 89, 92, 95 - epandrium, cercal plate and surstyli, lateral view; 90, 93, 96 - same, dorsal view. 89, 90 - after Ozerov, 2013, figs 17, 18; 94-96 - after Ozerov, 2010b, figs 27, 28, 30.

Pис. 88-96. Scathophaga litorea (Fallén) (88-90), S. lutaria (Fabricius) (91-93) и S. mollis (Becker) (94-96): 88, 91, 94 — стернит 5 самца; 89, 92, 95 - эпандрий, церки и сурстили, сбоку; 90, 93, 96 - то же, сверху. 89, 90 — по Ozerov, 2013, figs 17, 18; 94-96 — по Ozerov, 2010b, figs 27, 28, 30 . 


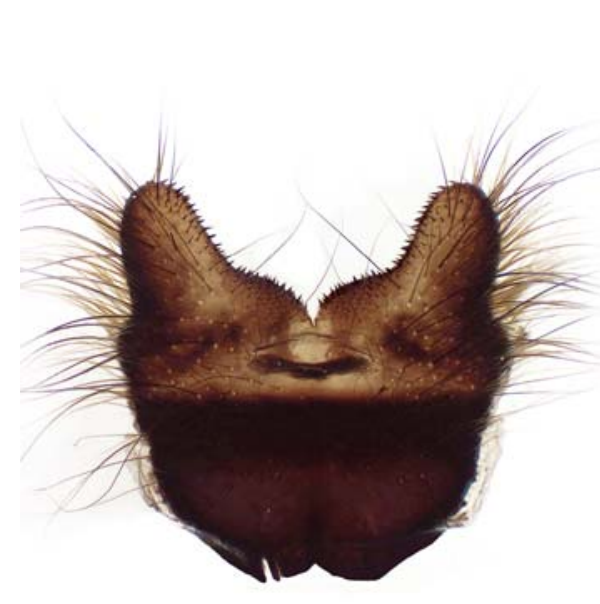

97
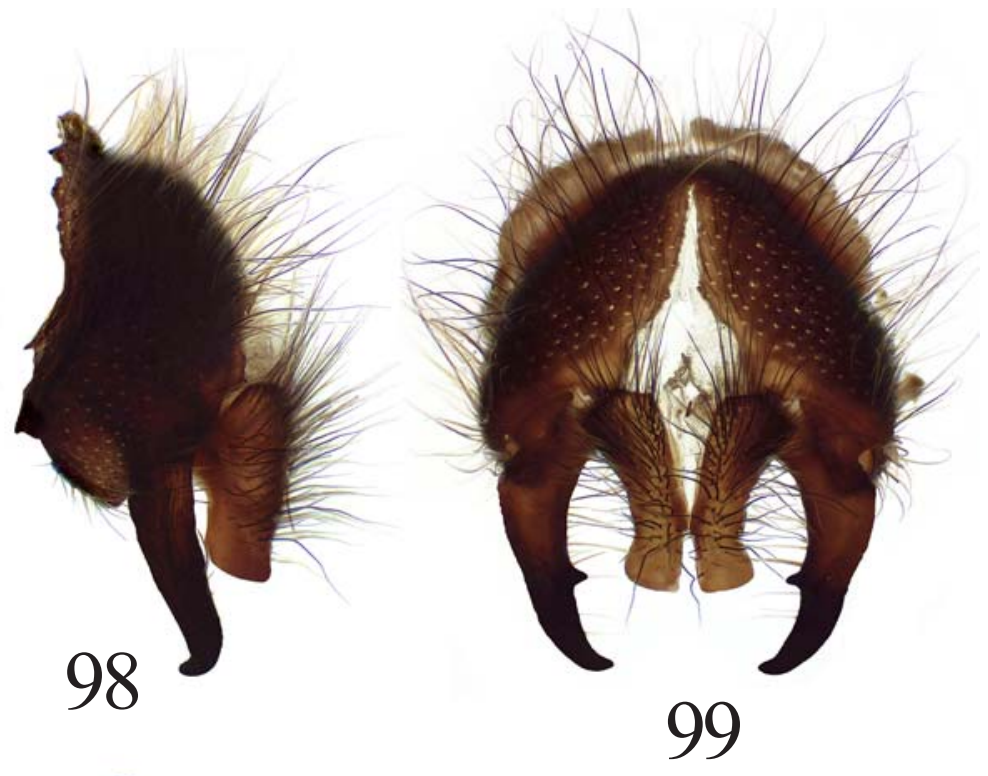

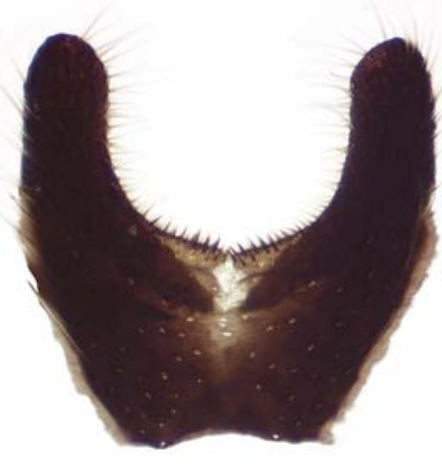

100

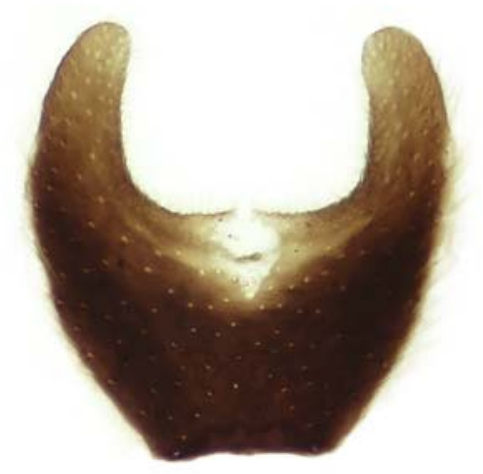

103
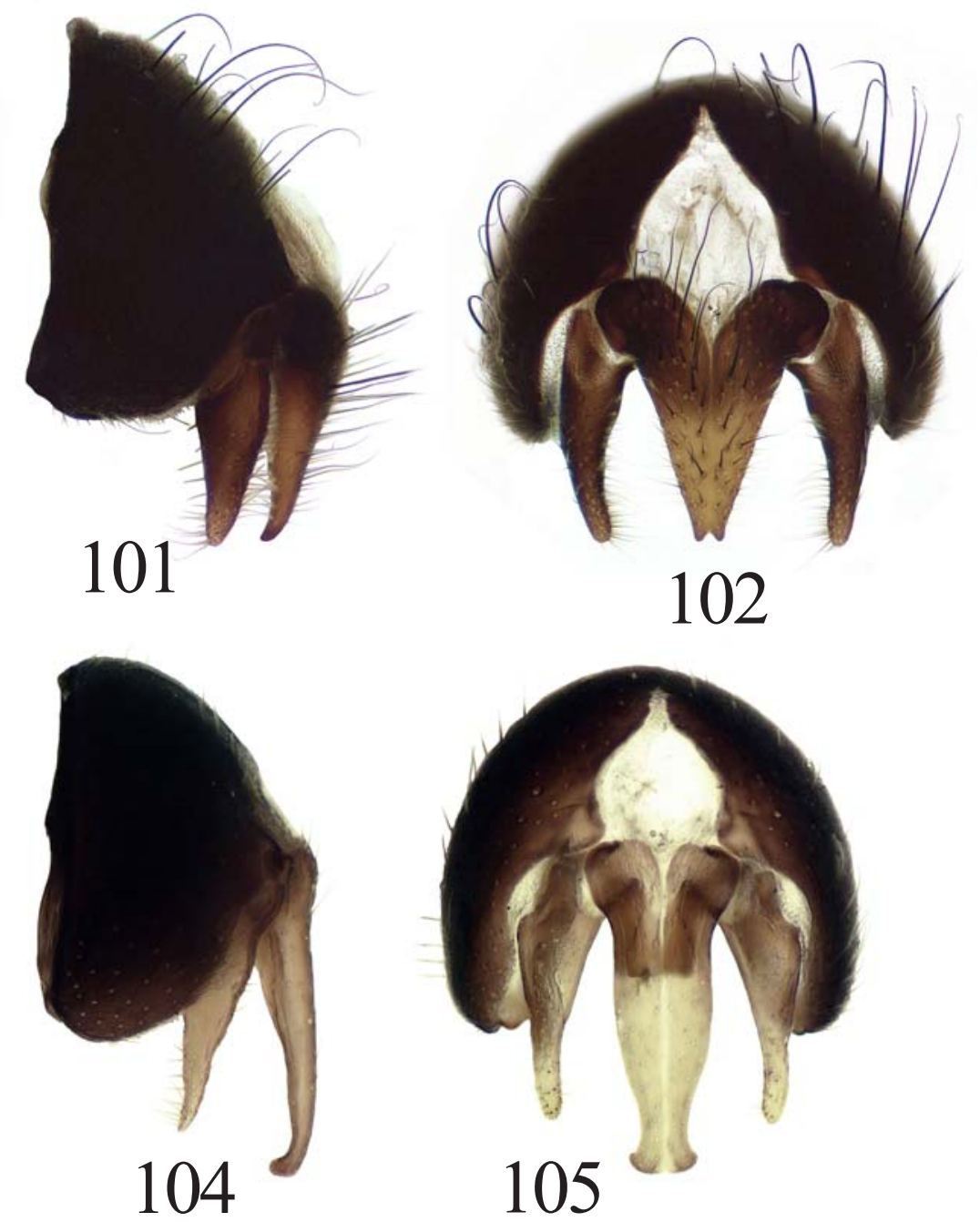

Figs 97-105. Scathophaga multisetosa (Holmgren) (97-99), S. nigripalpis (Becker) (100-102) and S. obscura (Fallén) (103-105): 97, 100, 103 - male sternite 5; 98, 101, 104 — epandrium, cercal plate and surstyli, lateral view; 99, 102, 105 - same, dorsal view.

Рис. 97-105. Scathophaga multisetosa (Holmgren) (97-99), S. nigripalpis (Becker) (100-102) и S. obscura (Fallén) (103-105): 97, 100, 103 - стернит 5 самца; 98, 101, 104 — эпандрий, церки и сурстили, сбоку; 99, 102, 105 — то же, сверху. 

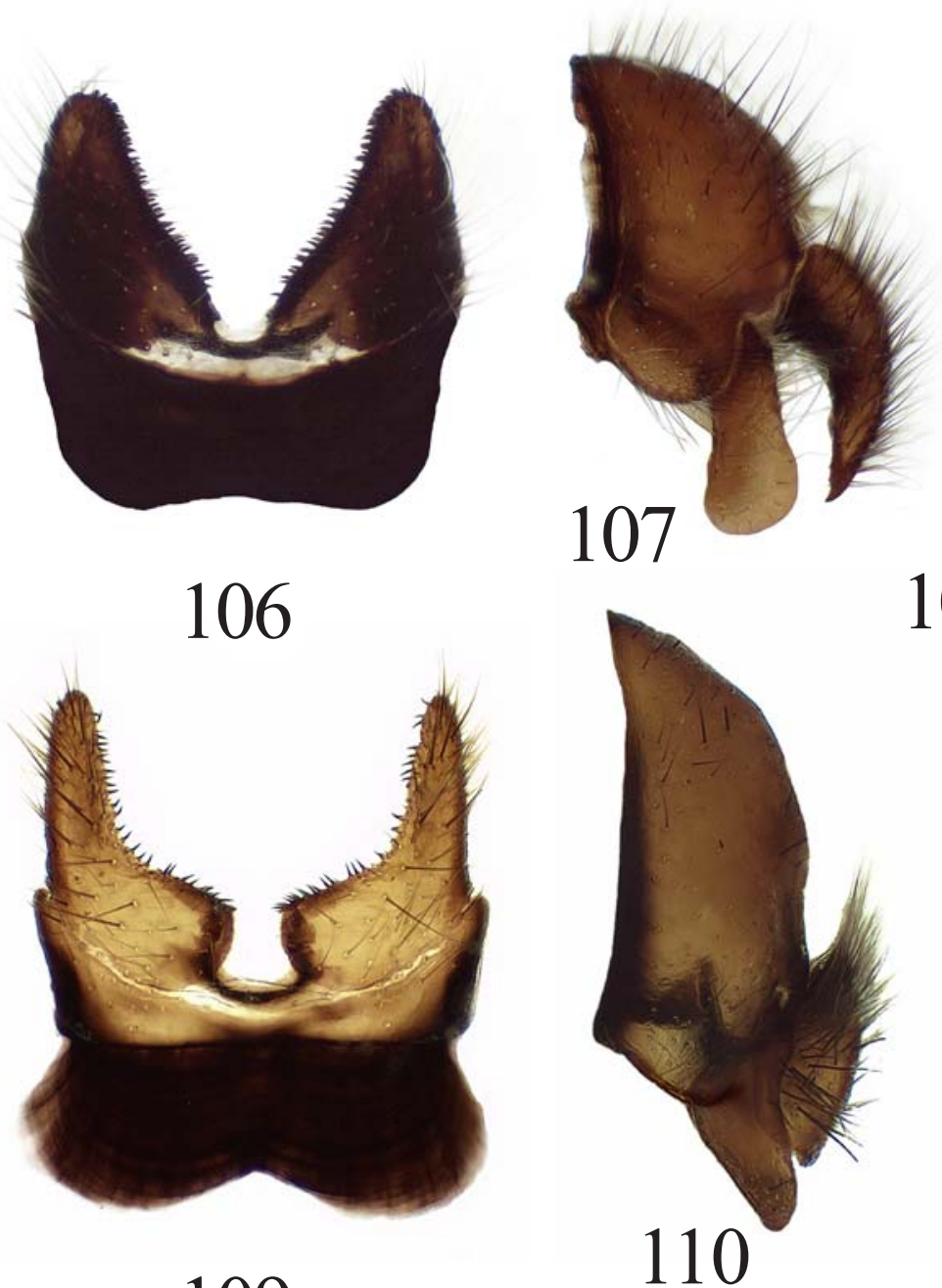

108

109
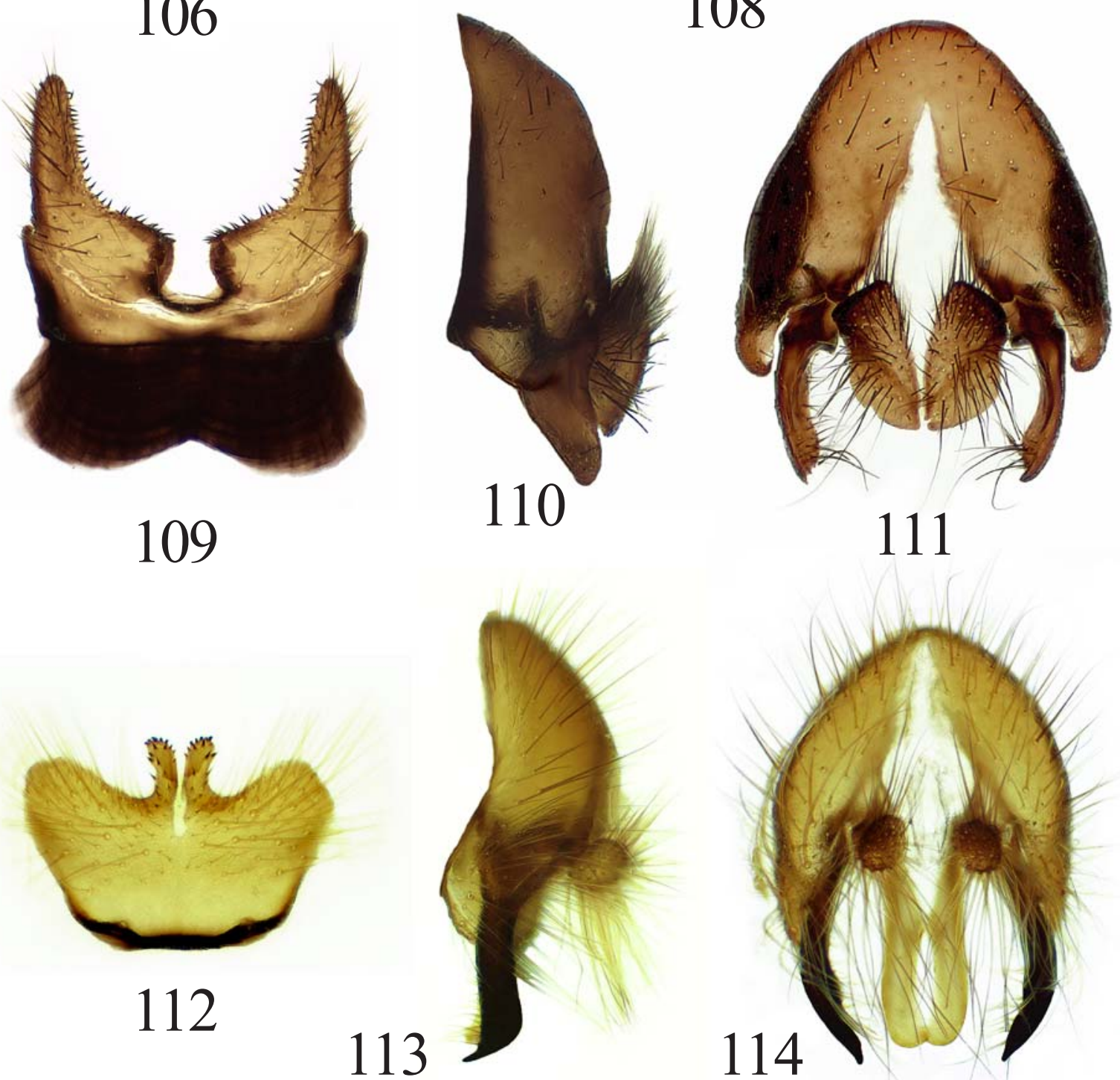

Figs 106-114. Scathophaga obscurinervis (Becker) (106-108), S. pictipennis (Oldenberg) (109-111) and S. spurca (Meigen) (112114): $106,109,112$ - male sternite $5 ; 107,110,113$ - epandrium, cercal plate and surstyli, lateral view; 108, 111, 114 - same, dorsal view. 109-111 — after Ozerov, 2017, figs 4-6.

Рис. 106-114. Scathophaga obscurinervis (Becker) (106-108), S. pictipennis (Oldenberg) (109-111) и S. spurca (Meigen) (112114): 106, 109, 112 - стернит 5 самца; 107, 110, 113 - эпандрий, церки и сурстили, сбоку; $108,111,114$ — то же, сверху. 109 111 — по Ozerov, 2017, figs 4-6. 
Legs yellow. Fore femur covered with hairs, but without conspicuous setae. Fore tibia with 2-3 dorsal, 0-1 posterior, 1 preapical dorsal, and 1 posterior apical setae. Mid femur with a row of anterior/anterodorsal setae, also with 1 preapical posterior and 1 preapical posterodorsal setae. Mid tibia with 1-3 posterodorsal, 1-2 anterodorsal setae and a ring of apicals. Hind femur with a row of anterodorsal setae. Hind tibia with 3 anterodorsal, 3 posterodorsal, 1 preapical dorsal, 1 preapical anterodorsal, and 1 apical anteroventral setae.

Wing tinged with brownish; crossveins r-m and dmcu not darkened or crossveins r-m slightly darkened only.

Abdomen from yellow to dark brownish, delicately greyish dusted, covered with not long hairs, in female tergites with black marginal setulae. Female tergite 7 greyish dusted. Male sternite 4 approximately 2 times as long as wide (Fig. 30); sternite 5 trapezoid with median cavity, but without apical processes and lateral lobes (Fig. 91). Epandrium, cercal plate and surstyli as in Figs 92, 93.

DISTRIBUTION. Russia: throughout midland, north to southern tundra. - Europe (widespread); known also from Algeria, Tunisia, Lebanon, Turkey and Israel [Šifner, 2008: 197; Ozerov, Freidberg, 2011: 175-176].

Scathophaga mollis (Becker, 1894) Figs 31, 94-96, 134.

mollis Becker, 1894: 171 (Scatophaga). Type-locality: "Sibiria" (Russia).

sychevskayae Ozerov, 2010b: 162, 165 (Scathophaga). Typelocality: Tabalach, Sakha [=Yakutia] (Russia).

REMARK. The species was recorded in Russia from Yakutia [Sychevskaya, 1972: 150; Ozerov, 2010b: 162, 165; Bagachanova et al. 2016: 782] and Magadan Oblast [Ozerov, Krivosheina, 2014: 218].

MATERIAL EXAMINED. Altai: River Chuya, $20 \mathrm{~km} \mathrm{NW}$ of Kosh-Agach $\left(50.085^{\circ} \mathrm{N} 88.404^{\circ} \mathrm{E}\right), 25-27$. VII.2006, A. Ovchinnikov, Nartshuk (3 90 , ZISP); Buryatia: Mondy env. $\left(51.675^{\circ} \mathrm{N}\right.$ $\left.100.992^{\circ} \mathrm{E}\right), 28$. VII.1965, Gorodkov (2 $\sigma^{7} \sigma^{7}, 1$ \%, ZISP); Irkutsk Oblast: Padun $\left(56.293^{\circ} \mathrm{N} 101.711^{\circ} \mathrm{E}\right)$, 4.IV.1867, Chekanovskiy (2 $\sigma^{\top} \sigma^{7}, 1$ + , ZISP); Kamchatka Krai: Mil'kovo $\left(54.697^{\circ} \mathrm{N}\right.$ $\left.158.620^{\circ} \mathrm{E}\right)$, 7.VII.1985, Zlobin (3 $\sigma^{7}, 1$ \%, ZISP); Magadan Oblast: Gizhiga $\left(61.948^{\circ} \mathrm{N} 160.377^{\circ} \mathrm{E}\right), 5-6 . \mathrm{IX} .1987$, Gorodkov (14 ${ }^{7}, 9$ 우, ZISP); Magadan env. (59.38 $\left.\mathrm{N} 150.51^{\circ} \mathrm{E}\right), 12 . \mathrm{VII} .2017$, V. Sorokina ( $\Im^{7} \sigma^{7}, 3$ o, ZISP); road Solnechnyy - Ola $\left(59.616^{\circ} \mathrm{N}\right.$ $\left.150.850^{\circ} \mathrm{E}\right), 13$.VIII. and 28.VIII.2017, N. Tridrikh (1 $\sigma^{\prime}, 4$ 우, ZMUM); Tuva: the upper reaches of the Mogun-Buren' River (ca. $\left.50.041^{\circ} \mathrm{N} 89.872^{\circ} \mathrm{E}\right), 26$. VII.1964, Nartshuk (1 $\sigma^{\top}$, ZISP); Yakutia: Abaga $\left(61.049^{\circ} \mathrm{N} 132.280^{\circ} \mathrm{E}\right), 19$.VII. 2008, A. Ovchinnikov (1 + , ZISP); airport Saskylakh (71.934 $\left.{ }^{\circ} \mathrm{N} 114.083^{\circ} \mathrm{E}\right), 24$. VII.1988, Gorodkov ( O $^{7}, 4$ ㅇ, ZISP); Balagannakh $\left(64.495^{\circ} \mathrm{N} 143.817^{\circ} \mathrm{E}\right)$ 4-8.VII.1974, Nartshuk (2 $\sigma^{7} \sigma^{7}, 7$ 우, ZISP); same place 24.VIII.1974, Gorodkov ( $8 \sigma^{7} \sigma^{7}, 7$ 우, ZISP); Batagay $\left(67.653^{\circ} \mathrm{N}\right.$ $\left.134.635^{\circ} \mathrm{E}\right)$, 9.VIII.1972, Gorodkov (4 $0^{7} \mathrm{O}^{7}, 3$ 오, ZISP); same place, 21.VII.1974, Gorodkov (5 O7 $\sigma^{7}, 7$ 우, ZISP); same place, 31.VII.2008, A. Ovchinnikov (12 $\sigma^{\top} \sigma^{7}, 8$ 우, ZISP); Deputatskiy $\left(69.319^{\circ} \mathrm{N} 139.966^{\circ} \mathrm{E}\right), 7 . V I I I .1974$, Gorodkov (11 ○ $^{7}, 8$ 우, ZISP); Khaptagay $\left(61.785^{\circ} \mathrm{N} 129.797^{\circ} \mathrm{E}\right), 4 . \mathrm{VII} .1974$, R. Kamenskaya (1 $\sigma^{7}$, ZMUM); Lake Tabalakh $\left(71.500^{\circ} \mathrm{N} 128.756^{\circ} \mathrm{E}\right)$, 3.VIII.1969, V. Sychevskaya (3 90 , ZMUM); left bank of the River Yana opposite of Verkhoyansk $\left(67.550^{\circ} \mathrm{N} 133.359^{\circ} \mathrm{E}\right), 23$. VII.1974, Nartshuk ( $8 \sigma^{7} \sigma^{7}, 13$ 우, ZISP); Mikhaylovka, $60 \mathrm{~km}$ NE of Amga (61.214 $\left.{ }^{\circ} \mathrm{N} 132.6815^{\circ} \mathrm{E}\right), 13-23$. VII.1985, 12-24.VIII.1985, $17-$ 23.VII. and 21.VIII.1986, Bagachanova, Novikov, Kaymuk (21 $\sigma^{7} \sigma^{\top}, 18$ 오, ZISP); Mirnyy $\left(62.540^{\circ} \mathrm{N} 113.962^{\circ} \mathrm{E}\right), 22 . \mathrm{VIII} .1988$, Gorodkov (11 $\sigma^{\top}, 8$ 우, ZISP); Neryuktyayinsk-1 $\left(60.258^{\circ} \mathrm{N}\right.$ $\left.119.673^{\circ} \mathrm{E}\right), 11,17$ and 18.VII. 2008, A. Ovchinnikov (12 $\sigma^{\top} \sigma^{\top}, 10$ OO, ZISP); Oktemtsy env. (61.672 $\left.{ }^{\circ} \mathrm{N} 129.417^{\circ} \mathrm{E}\right)$, 6.VII. 2008, A. Ovchinnikov ( $\sigma^{7} \sigma^{7}, 2$ 우, ZISP); Olekminsk $\left(60.370^{\circ} \mathrm{N}\right.$ $\left.120.436^{\circ} \mathrm{E}\right), 29$. VIII.1988, Gorodkov (11 $\sigma^{7} \sigma^{7}, 10$ 우, ZISP); Pokhodsk $\left(69.079^{\circ} \mathrm{N} 160.961^{\circ} \mathrm{E}\right), 18$.VII.1973, Vinokurov $\left(8 \bigcirc^{7} \sigma^{7}\right.$, 8 우, ZISP); River Tyung $\left(65.75^{\circ} \mathrm{N} 118.93^{\circ} \mathrm{E}\right), 6$. VIII.1926, Grigor'ev (1 9 , ZISP); River Yana, Stolby env. $\left(67.531^{\circ} \mathrm{N}\right.$

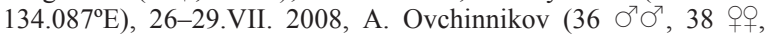
ZISP); Teply Klyuch $\left(62.783^{\circ} \mathrm{N} 136.809^{\circ} \mathrm{E}\right), 20-21$.VIII.1974, Gorodkov (14 $\mathrm{O}^{\top} \mathrm{O}^{\top}, 10$ 우, ZISP); Tolon $\left(59.458^{\circ} \mathrm{N} 111.539^{\circ} \mathrm{E}\right)$, 15.VII.1987, Zlobin (4 Ơ $\sigma^{\top}, 4$ 우, ZISP); Tyubelyakh env. $\left(65.371^{\circ} \mathrm{N} 143.1673^{\circ} \mathrm{E}\right), 11 . \mathrm{VII} .1974$, Nartshuk (1 ア, 4 90, ZISP); Tyungyulyu $\left(62.201^{\circ} \mathrm{N} 130.676^{\circ} \mathrm{E}\right), 26 . \mathrm{VII} .1996$, Bagachanova (1 $\sigma^{7}$, ZISP); Udachny $\left(66.405^{\circ} \mathrm{N} 112.299^{\circ} \mathrm{E}\right), 16-20$.VIII.1988, Gorodkov (4 O $\sigma^{7}, 4$ 우, ZISP); Ust'-Kuyga $\left(70.000^{\circ} \mathrm{N} 135.562^{\circ} \mathrm{E}\right), 26-$

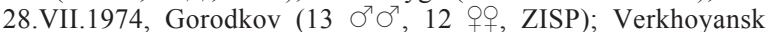
$\left(67.548^{\circ} \mathrm{N} 133.396^{\circ} \mathrm{E}\right), 23$.VII.1974, Gorodkov ( $9 \sigma^{\top} \sigma^{\top}, 8$, 8 , , ZISP); Yakutsk env. $\left(62.042^{\circ} \mathrm{N} 129.708^{\circ} \mathrm{E}\right), 23$.VII.1969, V. Sychevskaya ( $1 \mathrm{O}^{\top}, 7$ 우, ZMUM); Yakutsk env. $\left(62.159^{\circ} \mathrm{N} 129.822^{\circ} \mathrm{E}\right)$, 13.VII.1976, Kovalev (1 9 , ZISP); same place, 10.VIII.1987, Zlobin $\left(1 \bigcirc^{7}\right.$, ZISP); Yakutsk env., Chochur-Muran $\left(62.034^{\circ} \mathrm{N}\right.$ $\left.129.618^{\circ} \mathrm{E}\right), 1-2$.VII. and 3.VIII.2008, A. Ovchinnikov $\left(15 \sigma^{7} \sigma^{7}\right.$, 27 우, ZISP); Zhigansk $\left(66.766^{\circ} \mathrm{N} 123.372^{\circ} \mathrm{E}\right), 15$. VIII.1973, Gorodkov (25 $\sigma^{7} \sigma^{7}, 16$ 우, ZISP); Zabaikalsky Krai: Ulyatuy $\left(51.168^{\circ} \mathrm{N}\right.$ $\left.116.227^{\circ} \mathrm{E}\right), 16$. VIII.2016, A. Medvedev (1 $\sigma^{7}$ ZMUM). See also Ozerov [2010b: 162, 165], Ozerov \& Krivosheina [2014: 218].

DESCRIPTION. Male. Female. Body-length 6.1$7.4 \mathrm{~mm}$.

Head. Frontal vitta yellow or reddish-yellow, with delicate whitish reflection; fronto-orbital plate blackish, densely greyish dusted. Face, parafacial and gena yellow, with whitish reflection. Postcranium blackish. 3 orbital and 5-6 frontal setae present. Antenna black; postpedicel about twice as long as wide. Arista bare. Palpus black completely or yellow in basal half and black apically.

Thorax black, densely greyish dusted. Acrostichals not differentiated from the other hairs on scutum, dorsocentrals $2+3$, intra-alars $1+1$ (anterior postsutural), supra-alars $1+2$, postpronotals 2 . Anepimeron covered with hairs. Scutellum greyish dusted, with a pair of strong basal scutellar and a pair of strong apical scutellar setae.

Legs. All coxae black, greyish dusted. All femora greyish dusted, black, except yellow apex. All tibiae and tarsi yellow. Male fore and hind femora and female fore femur covered with whitish hairs, but without conspicuous setae. Fore tibia with 2 dorsal, 2 posterior, 1 preapical anterodorsal, and 1 posterior apical setae. Mid femur with 1 preapical posterior and 1 preapical posterodorsal setae in both sexes, additionally with a row of anterior setae in apical half in female. Mid tibia with 2-3 anterodorsal, 2 posterodorsal, 2-3 posterior, $0-1$ anteroventral, 1 ventral setae, also with a ring of apicals. Hind femur with a row of anterodorsal setulae. Hind tibia with 2 posterodorsal, 2 anterodorsal, 1 preapical dorsal, 1 preapical anterodorsal and 1 apical anteroventral setae. All setae on tibiae more or less strong in female and hair-like in male.

Wing clear; crossveins r-m and dm-cu not darkened or crossveins r-m slightly darkened only.

Abdomen black, densely pale grey dusted, covered with short whitish hairs. Tergites $2-5$ in male and tergi- 
tes 2-6 in female each with a row of marginal setulae (males) or setae (females). Male sternite 4 about as long as wide (Fig. 31). Male sternite 5 with short and narrow lobes (Fig. 94). Cercal plate short, not longer than short surstyli (Figs 95, 96). Aedeagus as in Fig. 134.

DISTRIBUTION. Russia: midland of Siberia and Far East. - Mongolia, China [Sun, 1996: 630], North America.

\section{Scathophaga multisetosa (Holmgren, 1883)} Figs 32, 97-99, 141.

multisetosa Holmgren, 1883: 174 (Scatomyza). Type-locality: "Norra Gåskap. Waigatsch" [Cape Severny Gusinyi Nos, Novaya Zemlya, Vaygach Island] (Russia).

REMARK. This species was recorded in high arctic territory of Russia: Novaya Zemlya, Vaygach I., New Siberian Islands, Taimyr Peninsula [Holmgren, 1883: 174; Gorodkov, 1986: 33; Ozerov, Barkalov, 2014: 563]. Incorrectly registered from Chukotka by Ozerov \& Krivosheina [2014: 218] (see above in $S$. apicalis).

MATERIAL EXAMINED. Chukotka: Shmidt $\left(68.892^{\circ} \mathrm{N}\right.$ 179.409 $\mathrm{W}$ ), 2.VII.1966, Gorodkov (6 $0^{7} \sigma^{7}, 4$ 우, ZISP); same place, 9-17.VII.1971, Gorodkov (35 $\sigma^{7} \sigma^{7}, 24$ 오, ZISP); Krasnoyarsk Krai: Dikson I. $\left(73.507^{\circ} \mathrm{N} 80.334^{\circ} \mathrm{E}\right)$, 9.VII.1961, Yu.I Chernov (12 $\sigma^{7} \sigma^{7}, 2$, 9, ZMUM); Taymyr, the lower reaches of the River Lenivaya $\left(74.966^{\circ} \mathrm{N} 89.916^{\circ} \mathrm{E}\right)$, on flowers of Sieversia glacialis, 4.VII.1980, Yu.I. Chernov (1 9 , ZMUM); Yakutia: Anabarsky Bay, Cape Khorgo $\left(73.502^{\circ} \mathrm{N} 113.551^{\circ} \mathrm{E}\right), 28 . \mathrm{VI} .1959$ (1 $\mathrm{O}^{7}$, ZMUM); Novosibirskie Islands, Kotel'nyy I. $\left(75.338^{\circ} \mathrm{N} 140.855^{\circ} \mathrm{E}\right)$, 14.VII.1973, Gorodkov (45 $\mathcal{O}^{7} \sigma^{7}, 28$ O+क, ZISP); Uryung-Khaya $\left(72.812^{\circ} \mathrm{N} 113.231^{\circ} \mathrm{E}\right)$, 5.VIII.1988, Gorodkov (1 $0^{7}$, ZISP). See also Ozerov \& Barkalov [2014: 563].

DESCRIPTION. Male. Female. Body-length 7.5$11.2 \mathrm{~mm}$.

Male thorax, abdomen and legs covered with dense, furry brownish hairs. Female much less hairy, especially abdomen and legs.

Head. Frontal vitta reddish-orange, with delicate whitish reflection; fronto-orbital plate black, greyish dusted. Face, parafacial and gena reddish-orange, with delicate whitish reflection. Postcranium black. 3 orbital and 6-8 frontal setae present. Scapus and pedicel reddish-orange. Postpedicel black, approximately 2 times as long as wide. Arista bare. Palpus yellow.

Thorax black, densely greyish dusted. Acrostichals, postpronotals and intra-alars not differentiated from the other long hairs on scutum, dorsocentrals $0+1$, supra-alars $(0-1)+(2-3)$, notopleurals $1-2$. Anepimeron bare. Scutellum black, greyish dusted, covered with dense hairs, with pairs of strong basal scutellar, lateral scutellar and apical scutellar setae.

Legs greyish dusted. Coxae black. Femora from yellow completely to blackish. Tibiae and tarsi yellowish. All femora and fore tibia covered with long hairs, but without conspicuous setae, only mid femur with 1 preapical posterior seta. Mid tibia with 2-3 posterodorsal, 2 anterodorsal setae, 1 posterior, 2 anteroventral, 1-2 posteroventral, 1-2 ventral setae and a ring of apicals. Hind tibia with 2-3 anterodorsal, 1 preapical dorsal, 1 apical anterodorsal, 1 apical anteroventral and 1 apical ventral setae; posterodorsal setae often absent in male or, as in female, not strong differentiated from the other long hairs on hind tibia (Fig. 141).

Wing tinged with brownish; crossveins r-m and dmcu darkened.

Abdomen black, greyish dusted; female tergites 2-6 each with a row of marginal setae. Male sternite 4 approximately as long as wide (Fig. 32), Male sternite 5, epandrium, cercal plate and surstyli as in Figs 97-99.

DISTRIBUTION. Russia: arctic islands and arctic coast from Taimyr to Chukotka. - North America; a littoral species.

Scathophaga nigripalpis (Becker, 1907)

Figs 5, 33, 100-102, 135.

nigripalpis Becker, 1907: 413 (Scatophaga). Type-locality: Ost-Grönland.

orbitalis Becker, 1915: 65 (Scopeuma). Type-locality: "Pemal" [hilly area between the northernmost parts of the Polar Urals and the Arctic sea coast] (Russia).

REMARK. This species was recorded in Russia for arctic zone of European part (e.g., Yugorsky Peninsula, Novaya Zemlya) and Siberia [Becker, 1915: 65; Gorodkov, 1970: 451, 1989: 33; Engelmark, 1999: 158, 159], from Yakutia [Bagachanova et al. 2016: 782] and Chukotka [Ozerov, Krivosheina, 2014: 218].

MATERIAL EXAMINED. Chukotka: Shmidt $\left(68.892^{\circ} \mathrm{N}\right.$ 179.409 $\mathrm{W})$, 17.VII.1963, Gorodkov (1 ア, 3 우, ZISP); Anadyr' $\left(64.731^{\circ} \mathrm{N} 177.504^{\circ} \mathrm{E}\right), 13$. VIII.1966, Gorodkov (25 o $\sigma^{7}, 16$ ZISP); Apapel'khin (ca. $\left.69.764^{\circ} \mathrm{N} 170.617^{\circ} \mathrm{E}\right), 21 . V I .1963$, Gorod-

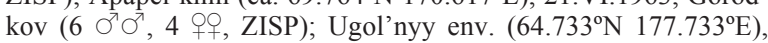

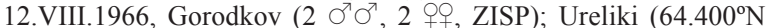
$\left.173.214^{\circ} \mathrm{W}\right), 3-6 . V I I I .1963$, Gorodkov (4 90 , ZISP); Yakutia: airport Saskylakh $\left(71.934^{\circ} \mathrm{N} 114.083^{\circ} \mathrm{E}\right), 24$.VII. and 9.VIII.1988, Gorodkov (6 $\sigma^{7} \sigma^{7}, 14$ 우, ZISP); Anabarsky Bay (ca. $73.502^{\circ} \mathrm{N}$ 113.55 $\left.{ }^{\circ} \mathrm{E}\right), 25$ and 30.VI.1959 (3 $\sigma^{7} \sigma^{7}$, ZMUM); Anabarsky Bay, Cape Khorgo $\left(73.504^{\circ} \mathrm{N} 113.525^{\circ} \mathrm{E}\right), 24-25$. VII.1959, Chernov (3 $\left.\mathrm{O}^{7} \sigma^{\top}, \mathrm{ZISP}\right)$; Chekurovka $\left(71.046^{\circ} \mathrm{N} 127.526^{\circ} \mathrm{E}\right)$, 24.VII.1957, Gorodkov (1 $\sigma^{7}$, ZISP); Chokurdakh $\left(70.618^{\circ} \mathrm{N} 147.895^{\circ} \mathrm{E}\right), 19$ 20.VIII.1971 and 7.VIII.1972, Gorodkov (20 $\sigma^{\top} \sigma^{7}, 18$ OO , ZISP); Kular $\left(70.573^{\circ} \mathrm{N} 134.271^{\circ} \mathrm{E}\right), 3$.VIII.1974, Gorodkov (1 $\left.{ }^{\circ}, \mathrm{ZISP}\right)$; Tiksi env. $\left(71.635^{\circ} \mathrm{N} 128.857^{\circ} \mathrm{E}\right), 2-17 . V I I I .1957$, Gorodkov (28 $\bigcirc^{7} \sigma^{\top}, 23$ 우, ZISP); Uryung-Khaya $\left(72.812^{\circ} \mathrm{N} 113.231^{\circ} \mathrm{E}\right), 26 . \mathrm{VII}$. and 7-8.VIII.1988, Gorodkov (6 O $\sigma^{7}, 5$ 우, ZISP); Kular $\left(70.573^{\circ} \mathrm{N}\right.$ $\left.134.271^{\circ} \mathrm{E}\right), 3-4$.VIII.1974, Gorodkov (10 $\sigma^{\top} \sigma^{7}, 8$ 우, ZISP); Nizhneyansk $\left(71.441^{\circ} \mathrm{N} 136.135^{\circ} \mathrm{E}\right), 31 . \mathrm{VII} .1974$, Gorodkov (3 $\sigma^{\top} \mathrm{O}^{7}, 3$ of, ZISP). See also Ozerov \& Krivosheina [2014: 218].

DESCRIPTION. Male. Female. Body-length 5.1$5.9 \mathrm{~mm}$.

Head. Frontal vitta yellow completely or blackish, but yellow in apical third or quarter, with delicate whitish reflection; fronto-orbital plate black, densely greyish dusted. Face, parafacial and gena yellow, with whitish reflection. Postcranium blackish. 4-6 frontals, 3 orbitals and additionally numerous setulae along margin of eye present (Fig.5). Antenna black; postpedicel about twice as long as wide. Arista bare. Palpus black (Fig.5).

Thorax black, greyish dusted, with dark stripes and fuzzy spots on scutum and scutellum. Acrostichals not differentiated from the other hairs on scutum, dorsocentrals $2+(2-3)$, intra-alars $1+0$, supra-alars $1+2$, postpronotals 2. Anepimeron with several hairs. Scutellum greyish dusted, with a pair of strong basal scutellar and a pair of strong apical scutellar setae. 
Legs. Coxae and femora of all legs black, greyish dusted. Tibiae and tarsi of all legs from yellow completely to black completely. Fore femur covered with long hairs, with 3-4 dorsal setae or setulae in apical third (female) or without conspicuous setae (male). Fore tibia with 2-4 dorsal, 2-4 posterodorsal, 1-2 posterior, 1 preapical dorsal, 1 preapical anterodorsal, and 1 posterior apical setae. Mid femur without conspicuous setae. Mid tibia with 1-2 anterodorsal, 1-3 posterodorsal, 0-1 anteroventral, 0-2 posterior setae, also with a ring of apicals. Hind femur with a row of anterodorsal setae. Hind tibia with 2-3 posterodorsal, 2-3 anterodorsal, 02 anteroventral, 1 preapical dorsal, 1 preapical anterodorsal and 1 apical anteroventral setae.

Wing clear; crossveins r-m and d-m not darkened.

Abdomen black, densely pale grey dusted, covered with whitish hairs; female tergites 2-6 each with a row of marginal setae. Male sternite 4 about 2 times as long as wide (Fig. 33). Male sternite 5 with moderately wide lateral lobes (Fig. 100). Cercal plate as long as surstyli (Figs 101, 102). Aedeagus as in Fig.135.

DISTRIBUTION. Russia: north from Polar Ural to Chukotka. — North America.

\section{Scathophaga obscura (Fallén, 1819) Figs 1, 34, 103-105, 136.}

obscura Fallén, 1819: 9 (Cordylura). Type-locality: "Scania... Lårketorp Ostrogothiae” (Sweden).

REMARK. The species was registered in Russia from Kola Peninsula [Gorodkov, 1970: 453], Caucasus and Kuril Islands [Gorodkov, 1986: 33], Siberia (Taimyr Peninsula) [Ozerov, Barkalov, 2014: 564] and Far East [Ozerov, Krivosheina, 2014: 218].

MATERIAL EXAMINED. Altai: Kosh-Agach env., $\left(50.01^{\circ} \mathrm{N}\right.$ $\left.88.66^{\circ} \mathrm{E}\right), 1750 \mathrm{~m}, 2-4$. VII.2016, N. Vikhrev (1 9 , ZMUM); KoshAgach, plateau Ukok, $2400 \mathrm{~m}$, surrounding area of Lake MuzdyBulak $\left(49.26^{\circ} \mathrm{N}, 87.65^{\circ} \mathrm{E}\right), 10$. VII.2008, A. Barkalov (1 $0^{7}$, ISEA); Onguday env., $\left(50.77^{\circ} \mathrm{N} 86.09^{\circ} \mathrm{E}\right), 8-13 . \mathrm{VII} .2016$, N. Vikhrev (3 $\left.\mathrm{O}^{7} \mathrm{O}^{\prime \prime}, \mathrm{ZMUM}\right)$; Seminsky pass, River Turala $\left(50.99^{\circ} \mathrm{N} 85.68^{\circ} \mathrm{E}\right)$, $1350 \mathrm{~m}, 8-12$. VII.2016, N. Vikhrev (1 $\sigma^{7}$, ZMUM); Tigirek Vill. $\left(51.13^{\circ} \mathrm{N} 83.05^{\circ} \mathrm{E}\right), 550 \mathrm{~m}, 9-12$. VII.2014, I. Shamshev (1 $\mathrm{O}^{\top}, 3$ 우, ZISP); Arkhangelsk Oblast: $73 \mathrm{~km} \mathrm{NW}$ of Nar'yan-Mar (ca. $\left.67.981^{\circ} \mathrm{N} 51.608^{\circ} \mathrm{E}\right)$, 3.VIII.1978, Gorodkov (1 $\mathrm{O}^{7}, 1$ ㅇ, ZISP); Nar'yan-Mar $\left(67.631^{\circ} \mathrm{N}, 52.985^{\circ} \mathrm{E}\right), 12 . \mathrm{VII} .2008$, A.L. Ozerov (1 $\mathrm{O}^{7}$, ZMUM); Solvychegovsk $\left(61.342^{\circ} \mathrm{N} 46.913^{\circ} \mathrm{E}\right), 12$ 13.VIII.2010, D. Gavryushin (4 $0^{7} O^{7}, 12$ 우, ZMUM); Bashkiria: between Abzakovo and Murakaevo (ca. $53.797^{\circ} \mathrm{N} 58.651^{\circ} \mathrm{E}$ ), 2-8.VIII.2008, K. Tomkovich (1 9 , ZMUM); Buryatia: the upper reaches of the River Irkut near Lake Il'chir $\left(51.961^{\circ} \mathrm{N} 100.952^{\circ} \mathrm{E}\right)$, 7.VIII.2012, A. Medvedev (1 $0^{7}, 1$ \%, ZMUM); Chelyabinsk Oblast: Taganay $\left(55.277^{\circ} \mathrm{N} 59.795^{\circ} \mathrm{E}\right), 18-24$.VII.2008, K. Tomkovich (2 $\sigma^{7} \sigma^{7}, 1$, ZMUM); Chukotka: Meynypil'gyno env. $\left(62.628^{\circ} \mathrm{N} 176.976^{\circ} \mathrm{E}\right), 14$.VII.2015, P.S. Tomkovich $\left(1 \sigma^{7}\right.$, ZMUM); Kamchatka Krai: Kozyrevsk $\left(56.048^{\circ} \mathrm{N} 159.870^{\circ} \mathrm{E}\right)$, 23.VII.1985, V. Zlobin (1 +, ZISP); Karelia: Primorsky $\left(66.555^{\circ} \mathrm{N}\right.$ 33.006 ${ }^{\circ}$ E), 5.VII.2010, A.L. Ozerov ( $7 \sigma^{7} \sigma^{7}, 4$ 우, ZMUM); Khabarovsk Krai: Bulgino env. $\left(59.371^{\circ} \mathrm{N} 143.147^{\circ} \mathrm{E}\right)$, 29.VIII.1987, Gorodkov (1 $\mathrm{O}^{7}$, ZISP); Khakassia: W Sayan pass $\left(51.717^{\circ} \mathrm{N} 89.882^{\circ} \mathrm{E}\right), 2100 \mathrm{~m}, 9-12 . \mathrm{VII} .2017, \mathrm{~N}$. Vikhrev (1 $\sigma^{7}$, ZMUM); Krasnodar Krai: Teberdinskiy Nature Reserve, $\left(43.443^{\circ} \mathrm{N} 41.738^{\circ} \mathrm{E}\right)$, 2.IX.1965, Gorodkov (1 $\sigma^{7}$, ZISP); Krasnoyarsk Krai: Igarka $\left(67.457^{\circ} \mathrm{N} 86.598^{\circ} \mathrm{E}\right)$, River Yenisei, 30.VI.1967, Gorodkov (4 $0^{7} \sigma^{7}, 2$ 우, ZISP); Igarka $\left(67.457^{\circ} \mathrm{N}\right.$ $86.598^{\circ} \mathrm{E}$ ), River Yenisei, 1.VII.1967, Gorodkov (1 9 , ZISP); Kryuchkovo Station $\left(56.096^{\circ} \mathrm{N} 92.109^{\circ} \mathrm{E}\right), 14-23 . \mathrm{VII} .2009$, K.
Tomkovich ( $1 \sigma^{7}$, ZMUM); Leningrad Oblast: Bol'shoy Berezovyy I. $\left(60.3^{\circ} \mathrm{N} 28.62^{\circ} \mathrm{E}\right)$, 7.VIII.1981, Kandybina (1 $\mathrm{O}^{\mathrm{T}}$, ZISP); Gor'kovskoe, Lake Lebyazh'e $\left(60.312^{\circ} \mathrm{N} 29.532^{\circ} \mathrm{E}\right), 24$.VIII.2003, A. Ovchinnikov (1 9 , ZISP); Luga (ca. $58.73^{\circ} \mathrm{N} 29.84^{\circ} \mathrm{E}$ ), 10.VII.1952, 27.VIII.1953, A. Stackelberg (1 O, 1 ㅇ, ZISP); Rozhdestveno $\left(59.322^{\circ} \mathrm{N} 29.946^{\circ} \mathrm{E}\right), 28$. VIII.1956, A. Stackelberg (1 $0^{7}$, ZISP); Yashchera $\left(58.894^{\circ} \mathrm{N} 29.820^{\circ} \mathrm{E}\right)$, 28.VIII. 1957 and 11.VII.1959, A. Stackelberg (4 $0^{7} 0^{7}, 2$ 우, ZISP); Moscow and Moscow Oblast: Dmitrov env. $\left(56.316^{\circ} \mathrm{N} 37.725^{\circ} \mathrm{E}\right), 7 . \mathrm{V} .2006$, N.Vikhrev (1 $\left.\mathrm{O}^{7}, \mathrm{ZMUM}\right)$; Ivanovskoe $\left(55.925^{\circ} \mathrm{N} 35.605^{\circ} \mathrm{E}\right)$, 17.VII.2007, A.L. Ozerov (1 $\bigcirc^{7}$, ZMUM); Izmaylovo $\left(55.800^{\circ} \mathrm{N}\right.$ $\left.37.832^{\circ} \mathrm{E}\right), 5 . \mathrm{VII} .1983$, A.L. Ozerov (3 $0^{7} \mathrm{O}^{7}, 3$ 우, ZMUM); NaroFominsk $\left(55.375^{\circ} \mathrm{N} 36.756^{\circ} \mathrm{E}\right), 8-13 . V .2006,14 . V ., 22 . V I .2010$,

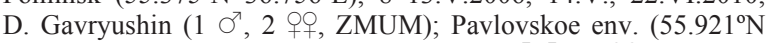

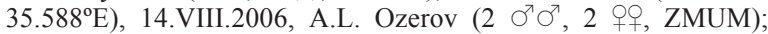
Rozhdestveno $\left(56.932^{\circ} \mathrm{N} 35.625^{\circ} \mathrm{E}\right)$, 17.VII.2007, A.L. Ozerov (1 $0^{7}, 1$ +, ZMUM); Yakshino $\left(55.922^{\circ} \mathrm{N} 35.577^{\circ} \mathrm{E}\right)$, 1.VIII.2005, A.L. Ozerov $\left(4 \mathrm{O}^{\top} \mathrm{O}^{7}, 4\right.$ 우, ZMUM); Zelenograd $\left(55.986^{\circ} \mathrm{N}\right.$ 37.202 ${ }^{\circ}$ E), 23.IV.1999, A. Gusakov (1 ${ }^{7}$, ZMUM); Murmansk Oblast: Molochnoe $\left(68.853^{\circ} \mathrm{N} 33.016^{\circ} \mathrm{E}\right), 20 . \mathrm{VII} .2011$, A. Ozerov (4 $\mathrm{O}^{7} \mathrm{O}^{7}, 1$ \% , ZMUM); Murmansk env. (68.979 $\left.\mathrm{N} 33.114^{\circ} \mathrm{E}\right)$, 21.VII.2011, A. Ozerov (1 9 , ZMUM); Revda $\left(67.942^{\circ} \mathrm{N} 34.552^{\circ} \mathrm{E}\right)$, 15.VIII.1981, Gorodkov ( $8 \mathrm{O}^{7} \mathrm{O}^{7}, 6$ 우 , ZISP); Novgorod Oblast: Terekhovo $\left(58.072^{\circ} \mathrm{N} 33.333^{\circ} \mathrm{E}\right), 29$.VIII.1948 (1 +, ZMUM); Novosibirsk Oblast: River Inya $\left(55.0^{\circ} \mathrm{N} 83.2^{\circ} \mathrm{E}\right), 22$. VII.2012, O. Kosterin (2 $\sigma^{7} \sigma^{7}$, ZMUM); Primorsky Krai: Gornotaezhnoe $\left(43.697^{\circ} \mathrm{N} 132.156^{\circ} \mathrm{E}\right)$, 5.IX.1980, Zlobin (1 + , ZISP); Lazovsky Nature Reserve, Proselochnaya Bay $\left(43.015^{\circ} \mathrm{N} 134.124^{\circ} \mathrm{E}\right), 3-$ 5.VII.2007, 6.VIII.2009, A. Ovchinnikov (3 $0^{7} \sigma^{7}, 2$ 우, ZISP); Suputinsky [=Ussuriysky] Nature Reserve (ca. $\left.46.591^{\circ} \mathrm{N} 137.021^{\circ} \mathrm{E}\right)$, 18.VIII.1980, Zlobin ( $1 \mathrm{O}^{7}, 1$ ㅇ, ZISP); Lazovsky Nature Reserve, cordon Korpad' $\left(43.263^{\circ} \mathrm{N} 134.131^{\circ} \mathrm{E}\right), 8-10$. VII.2007, 6.VIII.2009, A. Ovchinnikov $\left(1 \sigma^{7}, 1\right.$ \%, ZISP); Preobrazhenie $\left(42.909^{\circ} \mathrm{N}\right.$ $\left.133.890^{\circ} \mathrm{E}\right)$, 16-17.VII.2007, A. Ovchinnikov (1 O', ZISP); Tigrovyy (=Tigrovoe) $\left(43.189^{\circ} \mathrm{N} 132.894^{\circ} \mathrm{E}\right), 22 . \mathrm{VIII} .1979$, Zinov'ev ( $1 \mathrm{O}^{\circ}$, ZISP); Vladivostok env., Sedanka $\left(43.213^{\circ} \mathrm{N} 131.960^{\circ} \mathrm{E}\right)$, 20.VI.19278, A. Stackelberg (1 $\sigma^{7}$, ZISP). Ryazan' Oblast: Zalesnoe $\left(54.969^{\circ} \mathrm{N} 41.327^{\circ} \mathrm{E}\right), 21-26 . V I I .2013$, N. Vikhrev $\left(1 \sigma^{7}\right.$, ZMUM); Sakhalin Oblast: Kunashir I., Golovnin Volcano $\left(43.841^{\circ} \mathrm{N} 145.504^{\circ} \mathrm{E}\right)$, 24.VII.1973, Kasparyan (1 $\mathrm{O}^{7}$, ZISP); Kunashir I., Tret'yakovo $\left(43.989^{\circ} \mathrm{N} 145.644^{\circ} \mathrm{E}\right), 7 . V I I I .1971$, Nartshuk (1 ○’, ZISP); same place, 3.VIII.1973, Kasparyan (1 ○', ZISP); Sakhalin I., Yuzhno-Sakhalinsk (46.954 $\left.{ }^{\circ} \mathrm{N} 142.736^{\circ} \mathrm{E}\right)$, 22.VII.1956, Violovich $\left(1 \mathrm{O}^{7}, 1\right.$, , ZISP); Tuva: W Sayan pass $\left(51.69^{\circ} \mathrm{N} 89.96^{\circ} \mathrm{E}\right)$, 1840 m, 9.VII.2017, N. Vikhrev (1 $\sigma^{7}$, ZMUM); Tyumen' Oblast: $75 \mathrm{~km}$ WSW of Samburg (ca. $67.035^{\circ} \mathrm{N} 76.541^{\circ} \mathrm{E}$ ), 7-9.VIII.1976, Gorodkov (6 $\sigma^{7} \sigma^{7}, 5$ OP , ZISP); Neroyka (ca. $64.57^{\circ} \mathrm{N} 59.67^{\circ} \mathrm{E}$ ), $600 \mathrm{~m}, 5$. VIII.1990, Malozemov (2 $0^{7} \mathrm{O}^{7}$, ZISP); Voronezh Oblast: Ramon' $\left(51.908^{\circ} \mathrm{N} 39.333^{\circ} \mathrm{E}\right), 15 . \mathrm{IX} .1978$, A. Shatalkin (1 $q$, ZMUM); Yakutia: Deputatskiy $\left(69.319^{\circ} \mathrm{N} 139.966^{\circ} \mathrm{E}\right), 6 . \mathrm{VIII} .1974$, Gorodkov (11 $\mathrm{O}^{7} \mathrm{O}^{7}, 10$ 우, ZISP). See also Ozerov \& Krivosheina [2014: 218] and Ozerov \& Barkalov [2014: 564].

DESCRIPTION. Male. Female (Fig. 1). Body-length 4.5-6.1 mm.

Head. Frontal vitta yellow or reddish-yellow, matt; fronto-orbital plate blackish, greyish dusted. Face, parafacial and gena yellow, with delicate whitish or golden reflection. Postcranium blackish. 3 orbital and 3-4 frontal setae present. Antenna black; postpedicel about twice as long as wide. Arista bare. Palpus yellow.

Thorax black, densely pale grey dusted. Acrostichals absent, dorsocentrals $2+3$, intra-alars absent, supraalars $1+2$, postpronotals $1-2$. Scutellum greyish dusted, with a pair of strong basal scutellar and a pair of strong apical scutellar setae.

Legs. All coxae black, greyish dusted. All femora greyish dusted, black, except yellow apex. All tibiae and tarsi yellow. Fore femur covered with whitish hairs, with $4-5$ dorsal setulae in apical half. Fore tibia with 1 
dorsal, 0-1 posterior, 1 preapical dorsal, and 1 posterior apical setae. Mid femur with 1 preapical posterior seta. Mid tibia with 1 anterodorsal, 1 posterodorsal, 0 1 anteroventral setae, also with a ring of apicals. Hind femur with a row of anterodorsal setulae. Hind tibia with 2 posterodorsal, 2 anterodorsal, 1 preapical dorsal, 1 preapical anterodorsal and 1 apical anteroventral setae.

Wing clear; crossveins r-m and d-m not darkened.

Abdomen black, densely pale grey dusted, covered with short whitish hairs, without marginal setae. Male sternite 4 about twice as long as wide (Fig. 34). Male sternite 5 with moderately long and narrow lateral lobes (Fig. 103). Cercal plate longer than surstyli and narrowing to a slender incurved tip (Figs 104, 105). Aedeagus as in Fig. 136.

DISTRIBUTION. Russia: throughout midland, north to southern tundra. - Mongolia, North America.

\section{Scathophaga obscurinervis (Becker, 1900)} Figs 35, 106-108.

obscurinervis Becker, 1900: 55 (Scatophaga). Type-locality: "Insel Nikander und Dudinka" [Dudinka, Nikandrovskie Islands; NW Dudinka, River Yenisey] (Russia: Krasnoyarsk Krai).

REMARK. This species was registered in Russia for arctic zone of European part (e.g., Dolgii I., Kolguev I.) [Gorodkov, 1986: 33; Engelmark, 1999: 158, 159; Ovchinnikov, Makarova, 2016: 217], Siberia [Becker, 1900: 55; Ozerov, Barkalov, 2014: 564] and Chukotka [Ozerov, Krivosheina, 2014: 218].

MATERIAL EXAMINED. Arkhangelsk Oblast: Kolguev I., Bugrino $\left(68.782^{\circ} \mathrm{N} 49.309^{\circ} \mathrm{E}\right), 20-22$.VIII.1970, Gorodkov (6 $\sigma^{7} \sigma^{7}$, 6 우, ZISP); Nar'yan-Mar (67.636 $\left.{ }^{\circ} \mathrm{N} 53.031^{\circ} \mathrm{E}\right), 3$.VIII.1978, Gorodkov (2 $\sigma^{7} \sigma^{7}, 1$, ZISP); River Silova-Yakha (ca. $68.558^{\circ} \mathrm{N}$ $\left.64.817^{\circ} \mathrm{E}\right), 80 \mathrm{~km} \mathrm{~N}$ of Khal'mer'yu, 19.VII.1961, Gorodkov (6 $\sigma^{\top 7} \sigma^{7}, 4$ 우, ZISP); Chukotka: Pevek $\left(69.702^{\circ} \mathrm{N} 170.298^{\circ} \mathrm{E}\right)$ 3.VII.1963, Gorodkov (1 ア, ZISP); Krasnoyarsk Krai: 104 km NNW of Noril'sk, River Nizhnyaya Agapa (ca. $70.097^{\circ} \mathrm{N} 86.688^{\circ} \mathrm{E}$ ), 26-27.VII.1973, Sukacheva \& Zherikhin (1 ऽ', ZISP); River Pyasina (ca. $72.359^{\circ} \mathrm{N} 90.582^{\circ} \mathrm{E}$ ), $90 \mathrm{~km} \mathrm{~S}$ of Tareya, 23.VII.1967, Gorodkov (2 O' $\sigma^{\top}$, ZISP); Ust'-Tareya $\left(73.252^{\circ} \mathrm{N} 90.596^{\circ} \mathrm{E}\right), 14$ 15.VII.1967, Gorodkov (15 $\sigma^{\top} \sigma^{\top}, 10$ 우, ZISP); Tyumen' Oblast: $75 \mathrm{~km}$ WSW of Samburg (ca. 67.035 $\mathrm{N} 76.541^{\circ} \mathrm{E}$ ), 7-9.VIII.1976, Gorodkov (24 $\sigma^{7} \sigma^{7}, 20$ 우, ZISP); $83 \mathrm{~km} \mathrm{~W}$ of Tazovskiy (ca. $\left.67.466^{\circ} \mathrm{N} 76.762^{\circ} \mathrm{E}\right)$, valley of the River Khaddute, 29.VIII.1977, Gorodkov (5 $\sigma^{7}, 4$ OO, ZISP); $87 \mathrm{~km} \mathrm{NW}$ of Tazovskiy (ca. $\left.67.522^{\circ} \mathrm{N} 77.807^{\circ} \mathrm{E}\right)$, floodplain of the Khariyanog River, 29.VII.1977, Gorodkov (4 $\sigma^{7} \sigma^{7}, 2$ 90 , ZISP); $90 \mathrm{~km}$ W of Samburg (ca. $\left.67.035^{\circ} \mathrm{N} 76.541^{\circ} \mathrm{E}\right), 28 . V I I .1977$, Gorodkov (6 $\sigma^{7} \sigma^{7}, 4$ of, ZISP) Lake Yambuto $\left(71.314^{\circ} \mathrm{N} 80.003^{\circ} \mathrm{E}\right)$, VI-VII.1908, B.M. Zhitkov (Exspedition I. Russk. geogr. obshchestva to Yamal in 1908) (1 $\sigma^{7}$, ZISP); Lake Yaroto-Puykovo $\left(70.766^{\circ} \mathrm{N} 75.750^{\circ} \mathrm{E}\right)$, VII-VIII.1908, B.M. Zhitkov (Exspedition I. Russk. geogr. obshchestva to Yamal in 1908) (1 $\sigma^{7}$, ZMUM); Tabotarka River Basin $\left(67.477^{\circ} \mathrm{N} 70.558^{\circ} \mathrm{E}\right)$, VII-VIII.1908, B.M. Zhitkov (Exspedition I. Russk. geogr. obshchestva to Yamal in 1908) (1 9 , ZISP); Yakutia: airport Saskylakh $\left(71.934^{\circ} \mathrm{N} 114.083^{\circ} \mathrm{E}\right), 24$.VII.1988, Gorodkov (3 우, ZISP); Chokurdakh $\left(70.618^{\circ} \mathrm{N} 147.895^{\circ} \mathrm{E}\right), 20$ 21.VII.1971, Gorodkov (1 $\sigma^{7}, 1$ \%, ZISP); Tiksi env. $\left(71.635^{\circ} \mathrm{N}\right.$ $\left.128.857^{\circ} \mathrm{E}\right), 9$.VII. and 10.VIII.1957, Gorodkov (1 9 , ZISP); Olenek-

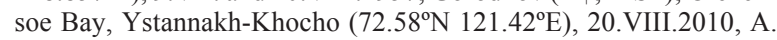
Yadrenkin (2 $9+$, ZMUM). See also Ozerov \& Krivosheina [2014: 218] and Ozerov \& Barkalov [2014: 564].

DESCRIPTION. Male. Female. Body-length 3.4$5.2 \mathrm{~mm}$.
Head. Frontal vitta yellowish-orange, matt; frontoorbital plate black, densely greyish dusted. Face, parafacial and gena yellow, with whitish reflection. Postcranium black, densely greyish dusted. 3 orbital and 23 frontal setae present. Scapus and pedicel reddish or black. Postpedicel black, approximately 2 times as long as wide. Arista bare. Palpus yellow.

Thorax black, densely greenish-grey dusted. Acrostichals setulose in two rows, dorsocentrals $2+3$, intraalars $1+2$, supra-alars $1+2$, postpronotals 2 . Anepimeron covered with hairs. Scutellum black, greenish-grey dusted, with a pair of strong basal scutellar and a pair of strong apical scutellar setae.

Legs delicately greyish dusted. All coxae black; fore femur yellow completely or blackish basally; mid and hind femora, all tibiae and all tarsi yellow. Male fore femur without conspicuous setae. Female fore femur with a row of dorsal/posterodorsal setulae. Fore tibia with 1-2 anterodorsal, 1-3 posterodorsal, 1 posterior, 1 preapical dorsal, and 1 posterior apical setae. Mid femur with 1 preapical posterior seta in both sexes, additionally with a row of anterior/anterodorsal setae in female. Mid tibia with 2 posterodorsal, 1-2 anterodorsal, 1-2 anteroventral/ventral, 1 posterior setae and a ring of apicals. Male hind femur without conspicuous setae. Female hind femur with a row of anterodorsal setae. Hind tibia with 2-3 anterodorsal, 2-3 posterodorsal, 1-2 anteroventral, 1 preapical dorsal, 1 preapical anterodorsal, 1 apical anterior , 1 apical anteroventral, and 1 apical ventral setae.

Wing tinged with brownish; crossveins r-m and d-m strongly darkened.

Abdomen black, greenish-grey dusted, covered with hairs in male; female tergites 2-6 with marginal setulae. Male sternite 4 wider than long (Fig. 35); sternite 5 with wide triangular lateral lobes, without median processes (Fig. 106). Epandrium, cercal plate and surstyli as in Figs 107, 108

DISTRIBUTION. Russia: arctic and subarctic from Arkhangelsk Oblast to Chukotka. - Europe (Scandinavia), North America; a rare northern holarctic species.

Scathophaga pictipennis (Oldenberg, 1923) Figs 36, 109-111.

pictipennis Oldenberg, 1923: 307 (Scatophaga). Type-locality: Gastein valley (Austria).

maculipenne Ringdahl, 1936: 175 (Scopeuma). Type-locality: "bei Idre im nördlichen Dalarna" (Sweden).

REMARK. The species is known for certain in Russia from the territory of Karelia only [Humala, Polevoi, 2009: 72].

MATERIAL EXAMINED. Karelia: Kivach Nature Reserve $\left(62.267^{\circ} \mathrm{N} 33.980^{\circ} \mathrm{E}\right), 31 . V$. and 5-8.VI.1989, A.V. Polevoy (1 ○', 1 , , ZMUM).

DESCRIPTION. Male. Female. Length of body 4.5$5.6 \mathrm{~mm}$.

Head. Frontal vitta yellow, with delicate whitish reflection; fronto-orbital plate blackish, greyish dusted. Face and gena yellow, with whitish reflection. Postcranium black in upper half and yellow in lower 
half. 3 orbital and 3-4 frontal setae present. Scapus and pedicel dark reddish. Postpedicel black, about twice as long as wide. Arista bare. Palpus yellow.

Thorax black, dense pale grey dusted, only postpronotal lobe yellowish. Acrostichals setulose in two rows, dorsocentrals $2+3$, intra-alars $1+2$, supra-alars $1+2$, postpronotals 2 . Anepimeron bare. Scutellum greyish dusted, with a pair of strong basal scutellar and a pair of strong apical scutellar setae.

Legs. Fore coxa yellow, mid and hind coxae brownish or blackish. Fore femur black, except yellow apex, greyish dusted. Mid and hind femora, all tibiae and all tarsi yellow. Male fore femur covered with whitish and blackish hairs, without conspicuous setae. Female fore femur with a row of dorsal/posterodorsal setae. Fore tibia with 2-3 dorsal, 1 posterior, 1 preapical anterodorsal, 1 apical posterodorsal, 1 apical posterior setae. Mid femur with a row of anterodorsal setae, with 1 preapical posterodorsal and 1 preapical posterior setae. Mid tibia with 2 anterodorsal, 2 posterodorsal, $0-1$ ventral setae, also with a ring of apicals. Hind femur with a row of dorsal/ anterodorsal setae. Hind tibia with $2-3$ posterodorsal, 2-3 anterodorsal, 2-3 anteroventral, 1 preapical dorsal, 1 preapical anterodorsal, and 2 apical anteroventral setae. ened.

Wing clear; crossveins r-m and d-m broadly dark-

Abdomen brownish, greyish dusted. Male sternite 4 slightly wider than long (Fig. 36). Male sternite 5 with broad in base narrow apically lateral lobes and with deep and wide median cavity (Fig. 109). Epandrium, cercal plate and surstyli as in Figs 110, 111.

DISTRIBUTION. Russia: Karelia. — Europe, North America; extremely rare species.

\section{Scathophaga spurca (Meigen, 1826)} Figs 37, 112-114.

Scatomyza suilla (Fabricius,); Fallén [1819: 5]. Misidentification [see: Michelsen, O’Hara, 2014: 77-79]. given.

spurca Meigen, 1826: 250 (Scatophaga). Type-locality: not

glabrata Zetterstedt, 1838: 721 (Scatomyza). Type-localities: "Lapponia Norvegica borealis — Dalekarlia Sueciae" (Norway, Sweden).

scatomyzoides Zetterstedt, 1838: 727 (Cordylura). Type-locality: "Lapponia Umensi... Wilhelmina... (Lappon. - meridional.)" (Sweden).

serotina Perris, 1839: 48 (Scatophaga). Type-locality: “environs de Mont-de-Marsan" (Landes, France).

REMARKS. This widespread and common species was recorded in Russia (in most cases as $S$. suilla) from Karelia [Polevoi, 1997: 309; Humala, Polevoi, 2009: 72], Kanin Peninsula [Engelmark, 1999: 158, 159], Mordovia [MacGowan et al., 2021: 17], Yaroslavl' Oblast [Ovchinnikov, 2004: 422], Krasnoyarsk Krai (Taimyr Peninsula) [Ozerov, Barkalov, 2014: 564], Yakutia [Sychevskaya, 1972: 150; Veselkin, 1985: 75; Bagachanova et al., 2016: 783] and Far East [Ozerov, Krivosheina, 2014: 219].

Gorodkov published a map with the distribution area of this species on the territory of the former USSR and separately on its European part [Gorodkov, 1980: map 68 , as $S$. suilla], but without the names of the points.

MATERIAL EXAMINED. Altai: River Chuya, $20 \mathrm{~km} \mathrm{NW}$ of Kosh-Agach $\left(50.085^{\circ} \mathrm{N} 88.404^{\circ} \mathrm{E}\right), 25-27$. VII.2006, Nartshuk (1 $0^{7}$, ZISP); Amur Oblast: Bol'shoy Never $\left(53.978^{\circ} \mathrm{N} 124.150^{\circ} \mathrm{E}\right)$, 7.IX.1974, Gorodkov (7 $\bigcirc^{7} \sigma^{7}, 5$ + , ZISP); Arkhangelsk Oblast: $70 \mathrm{~km} \mathrm{~N}$ Nar'yan-Mar (ca. $68.204^{\circ} \mathrm{N} 53.627^{\circ} \mathrm{E}$ ), 18.VIII.1978, Gorodkov (4 $\mathrm{O}^{7} \mathrm{O}^{7}, 1$ + , ZISP); $73 \mathrm{~km} \mathrm{NW}$ of Nar'yan-Mar (ca. $\left.67.981^{\circ} \mathrm{N} 51.608^{\circ} \mathrm{E}\right), 3$. VIII.1978, Gorodkov (3 $0^{7} \mathrm{O}^{7}, 1$ ㅇ, ZISP); Arkhangelsk $\left(64.546^{\circ} \mathrm{N} 40.567^{\circ} \mathrm{E}\right), 10 . \mathrm{VIII} .1967$, Gorodkov $\left(8 \mathrm{O}^{7} \mathrm{O}^{\mathrm{T}}\right.$, 3 우, ZISP); Belush'e env. (66.89 $\left.{ }^{\circ} \mathrm{N} 47.61^{\circ} \mathrm{E}\right), 24$.VIII.1978, Gorodkov (2 O $^{7} \sigma^{7}$, ZISP); Kanin Nos $\left(68.656^{\circ} \mathrm{N} 43.279^{\circ} \mathrm{E}\right), 17 . \mathrm{VII} .1970$, Gorodkov (4 $\sigma^{\top} \sigma^{7}, 3$ 우, ZISP); Kargopol' $\left(61.503^{\circ} \mathrm{N} 38.971^{\circ} \mathrm{E}\right)$, 13.VII.1982, Gorodkov ( 1 +, ZISP); Kolguev I., Bugrino $\left(68.782^{\circ} \mathrm{N}\right.$ $\left.49.309^{\circ} \mathrm{E}\right), 20-21 . \mathrm{VIII} .1970$, Gorodkov (3 $\mathrm{O}^{\mathrm{T}} \mathrm{O}^{7}, 3$ OP, ZISP); Lomonosovo $\left(64.229^{\circ} \mathrm{N} 41.732^{\circ} \mathrm{E}\right)$, 31.VIII.1978, Gorodkov (1 $\sigma^{7}$, ZISP); Mezen' (65.85 $\left.\mathrm{N} 44.24^{\circ} \mathrm{E}\right), 25$ and 27.VIII.1978, Gorodkov (3 $\sigma^{\prime \prime} \sigma^{7}, 2$ 우, ZISP); Nar'yan-Mar $\left(67.636^{\circ} \mathrm{N} 53.031^{\circ} \mathrm{E}\right)$, 28.VIII.1970, 4, 7 and 21.VIII.1978, Gorodkov (5 $0^{7} 0^{7}, 3$ 우, ZISP); Nizhnyaya Pesha $\left(66.751^{\circ} \mathrm{N} 47.760^{\circ} \mathrm{E}\right), 22$.VIII.1978, Gorodkov $\left(10^{7}, 1\right.$ ㅇ, ZISP); Onega $\left(63.922^{\circ} \mathrm{N} 38.085^{\circ} \mathrm{E}\right), 20 . \mathrm{VII} .1996$, Gorodkov (2 ${\sigma^{7}}^{7} \sigma^{7}$, ZISP); Solovetskiy I. $\left(65.080^{\circ} \mathrm{N} 35.686^{\circ} \mathrm{E}\right)$, 27.VII.1975, Gorodkov (1 $\mathrm{O}^{7}$, ZISP); Solvychegovsk env. (61.327 $\mathrm{N}$ $\left.46.918^{\circ} \mathrm{E}, 61.342^{\circ} \mathrm{N} 46.913^{\circ} \mathrm{E}\right), 13-14$. VIII.2010, D. Gavryushin

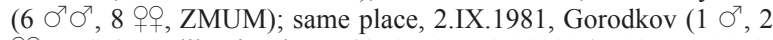
우, ZISP); Velikovisochnoe $\left(67.255^{\circ} \mathrm{N} 52.0328^{\circ} \mathrm{E}\right), 18 . \mathrm{VIII} .1978$, Gorodkov ( $2 \bigcirc^{T} \sigma^{7}, 3$ 우, ZISP); Bashkiria: Bashkirskiy Nature Reserve $\left(53.420^{\circ} \mathrm{N} 57.935^{\circ} \mathrm{E}\right)$, 7.VIII.1975, Gorodkov (4 $\mathrm{O}^{7} \mathrm{O}^{7}, 2$ 우, ZISP); Beloretsk env. $\left(54.051^{\circ} \mathrm{N} 58.268^{\circ} \mathrm{E}, 54.004^{\circ} \mathrm{N} 58.463^{\circ} \mathrm{E}\right.$, $\left.53.973^{\circ} \mathrm{N} 58.344^{\circ} \mathrm{E}\right), 7-10$. VIII.2012, D. Gavryushin (2 $\mathrm{O}^{\top} \mathrm{O}^{\top}, 1$, ZMUM); Ufa env. ( $\left.54.776^{\circ} \mathrm{N} 56.068^{\circ} \mathrm{E}\right)$, 18.VIII.1975, Gorodkov ( $2 \sigma^{\top} \sigma^{7}, 1$ \% , ZISP); Buryatia: Barguzinsky Nature Reserve (ca. $\left.54.35^{\circ} \mathrm{N} 109.51^{\circ} \mathrm{E}\right), 20$. VII.1965, Negrobov (2 $\mathrm{O}^{7} \mathrm{O}^{\top}, 1$ ㅇ, ZISP); Khasura $\left(50.327^{\circ} \mathrm{N} 103.282^{\circ} \mathrm{E}\right)$, 1.VII.1971, V. Richter (1 O', ZISP); Tankhoy $\left(51.554^{\circ} \mathrm{N} 105.118^{\circ} \mathrm{E}\right), 10-17 . V I .1980$, Barkalov (1 O, 1 + , ZISP); Chukotka: Markovo (ca. $64.680^{\circ} \mathrm{N} 170.412^{\circ} \mathrm{E}$ ), 18.VIII.1966, Gorodkov (10 $\sigma^{7} \sigma^{7}, 8$ 우, ZISP); Irkutsk Oblast: Padun $\left(56.293^{\circ} \mathrm{N} 101.711^{\circ} \mathrm{E}\right), 24 . V I .1956$, Monchadskiy (13 $\mathrm{O}^{7} \sigma^{7}$, 8 웅, ZISP); Kamchatka Krai: Kozyrevsk $\left(56.048^{\circ} \mathrm{N} 159.870^{\circ} \mathrm{E}\right)$, 7.IX.1969, Gorodkov (2 $\sigma^{7} \sigma^{7}, 1$ 우, ZISP); Commander Islands,

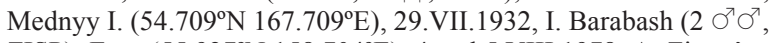
ZISP); Esso $\left(55.927^{\circ} \mathrm{N} 158.704^{\circ} \mathrm{E}\right), 4$ and 5.VIII.1978, A. Zinov'ev $\left(20^{7} \mathrm{O}^{7}\right.$, ZISP); Commander Islands, Preobrazhenskoe $\left(54.790^{\circ} \mathrm{N}\right.$ $\left.167.578^{\circ} \mathrm{E}\right)$, 5.IX.1959, Gorodkov (2 $\sigma^{7} \sigma^{7}, 1$, , ZISP); Karelia: Gridino $\left(65.920^{\circ} \mathrm{N} 34.669^{\circ} \mathrm{E}\right), 11 . I X .1984$, Gorodkov (1 9 , ZISP); Kartesh $\left(66.33^{\circ} \mathrm{N} 33.64^{\circ} \mathrm{E}\right), 18$ and 21.VII.1975, Gorodkov (4 $\mathrm{O}^{7} \mathrm{O}^{\prime \prime}$, 3 우, ZISP); Keret' $\left(66.190^{\circ} \mathrm{N} 32.884^{\circ} \mathrm{E}\right)$, 8.IX.1984, Gorodkov (1 $0^{7}, 1$ \%, ZISP); Pudozh $\left(66.804^{\circ} \mathrm{N} 36.565^{\circ} \mathrm{E}\right), 17$ and 19.VII.1982, Gorodkov ( $5 \bigcirc^{7} O^{7}, 2$ 우, ZISP); Khabarovsk Krai: Bulgino env. $\left(59.371^{\circ} \mathrm{N} 143.147^{\circ} \mathrm{E}\right), 29$. VIII.1987, Gorodkov $\left(2 \mathrm{O}^{7} \mathrm{O}^{7}, 1\right.$, 1 , ZISP); Khakassia: W Sayan pass $\left(51.717^{\circ} \mathrm{N} 89.882^{\circ} \mathrm{E}\right), 2100 \mathrm{~m}, 9-$ 12.VII.2017, N. Vikhrev (6 O $^{7} \sigma^{7}, 1$, ZMUM) Komi: Blagoevo $\left(63.41^{\circ} \mathrm{N} 47.95^{\circ} \mathrm{E}\right), 12$. VII.1996, Gorodkov (1 O $\mathrm{O}^{\prime \prime}$, ZISP); Ust-Tsilma $\left(65.440^{\circ} \mathrm{N} 52.153^{\circ} \mathrm{E}\right)$, 9.VIII.1978, Gorodkov (2 $0^{\circ} \mathrm{O}^{7}$, ZISP); Yaksha $\left(61.823^{\circ} \mathrm{N} 56.821^{\circ} \mathrm{E}\right), 12$. VIII.1989, Gorodkov $\left(10 \mathrm{O}^{7} \mathrm{O}^{7}, 4\right.$ 우, ZISP); Krasnoyarsk Krai: Baykit $\left(61.68^{\circ} \mathrm{N} 96.38^{\circ} \mathrm{E}\right), 23-$

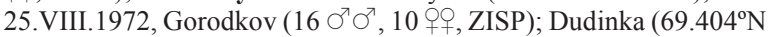
$86.182^{\circ} \mathrm{E}$ ), 2-3.VII.1967, Gorodkov (2 $\sigma^{7} \sigma^{7}$, ZISP); Igarka $\left(67.457^{\circ} \mathrm{N} 86.598^{\circ} \mathrm{E}\right)$, River Yenisei, 30.VI. and 1.VII. 1967, Gorodkov (4 $\bigcirc^{7} \sigma^{\top}$, ZISP); Khatanga $\left(71.981^{\circ} \mathrm{N} 102.483^{\circ} \mathrm{E}\right), 25$. VIII.1971, Gorodkov (13 $\bigcirc^{7} \sigma^{\prime}, 8$ 우, ZISP); Turuchansk $\left(65.797^{\circ} \mathrm{N} 87.958^{\circ} \mathrm{E}\right)$, 27-28.VI.1967, Gorodkov (6 $0^{7} \sigma^{7}, 4$ ㅇ․, ZISP); Leningrad Oblast: Luga (ca. $\left.58.73^{\circ} \mathrm{N} 29.84^{\circ} \mathrm{E}\right), 15$.VII.1953, 16.VIII.1955, A. Stackelberg (1 $\mathrm{O}^{\mathrm{T}}, 2$ 우, ZISP); Rakovichi $\left(58.650^{\circ} \mathrm{N} 29.836^{\circ} \mathrm{E}\right), 7$ and 31.V., 13-15.VI.1897, G. Pleske (5 $0^{\top} 0^{\top}, 2$ 우, ZISP); Saint Petersburg $\left(59.934^{\circ} \mathrm{N} 30.359^{\circ} \mathrm{E}\right)$, 29.VIII.1995, Gorodkov (6 $\sigma^{7} \sigma^{7}$, 1 O Z ZISP); Tolmachevo $\left(58.856^{\circ} \mathrm{N} 29.895^{\circ} \mathrm{E}\right), 11 . \mathrm{VI}$. and 7.VII.1935, Rohdendorf (5 $\mathrm{O}^{7} \mathrm{O}^{\top}, 2$ 우, ZISP); Yashchera (58.894 ${ }^{\circ} \mathrm{N}$ $\left.29.820^{\circ} \mathrm{E}\right)$, 7.VI.1958, A. Stackelberg (1 O', ZISP); Lipetsk Oblast: Donskoe $\left(52.618^{\circ} \mathrm{N} 38.978^{\circ} \mathrm{E}\right)$, 29.VIII.1978, Tryapitsyn $(1$ $\mathrm{O}^{7}$, ZISP); Magadan Oblast: Chaybukha $\left(61.80^{\circ} \mathrm{N} 160.413^{\circ} \mathrm{E}\right)$, 1.IX.1987, Gorodkov (2 $\sigma^{7} \mathrm{O}^{7}, 7$ Oㅇ, ZISP); Evensk $\left(61.916^{\circ} \mathrm{N}\right.$ $\left.159.234^{\circ} \mathrm{E}\right)$, 9.IX.1987, Gorodkov (1 $\mathrm{O}^{7}$, ZISP); Gizhiga $\left(61.948^{\circ} \mathrm{N}\right.$ 


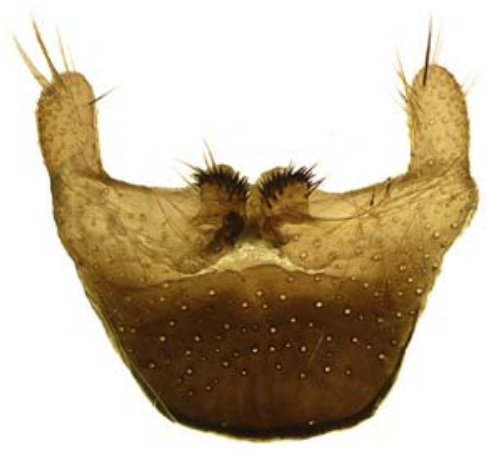

115

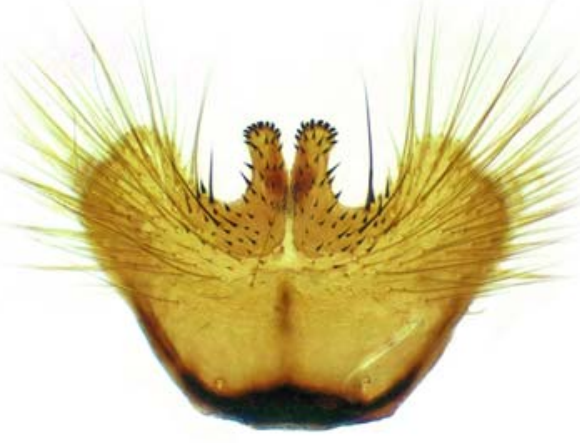

118

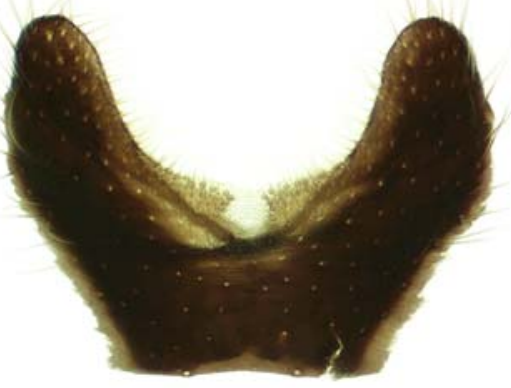

121

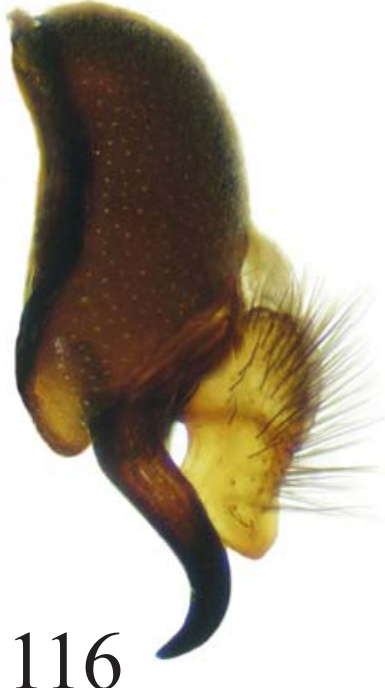

116
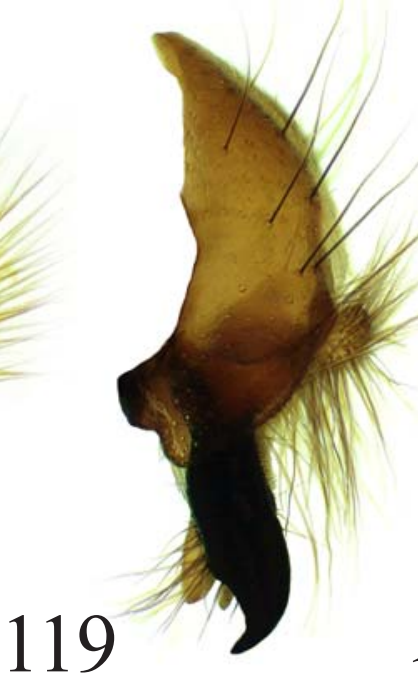

120
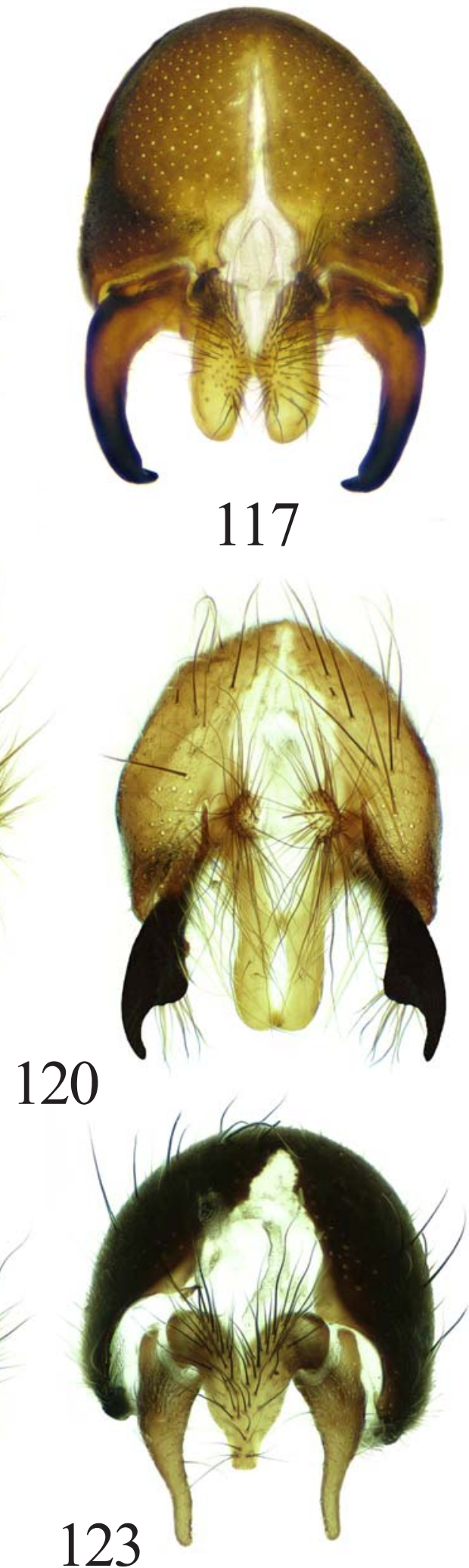

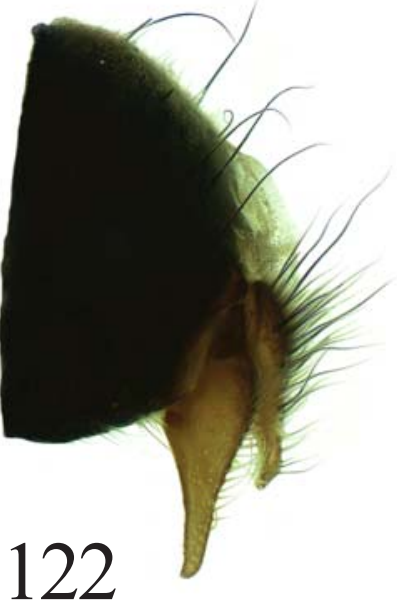

123

Figs 115-123. Scathophaga stercoraria (Linnaeus) (115-117), S taeniopa (Rondani) (118-120) and S tinctinervis (Becker) (121-123): $115,118,121$ - male sternite 5; 116, 119, 122 - epandrium, cercal plate and surstyli, lateral view; 117, 120, 123 - same, dorsal view. 116, 117 - after Ozerov \& Freidberg, 2011, figs 25, 26.

Рис. 115-123. Scathophaga stercoraria (Linnaeus) (115-117), S. taeniopa (Rondani) (118-120) и S. tinctinervis (Becker) (121-123): $115,118,121$ - стернит 5 самца; 116, 119, 122 - эпандрий, церки и сурстили, сбоку; 117, 120, 123 - то же, сверху. 116,117 - по Ozerov \& Freidberg, 2011, figs 25, 26. 

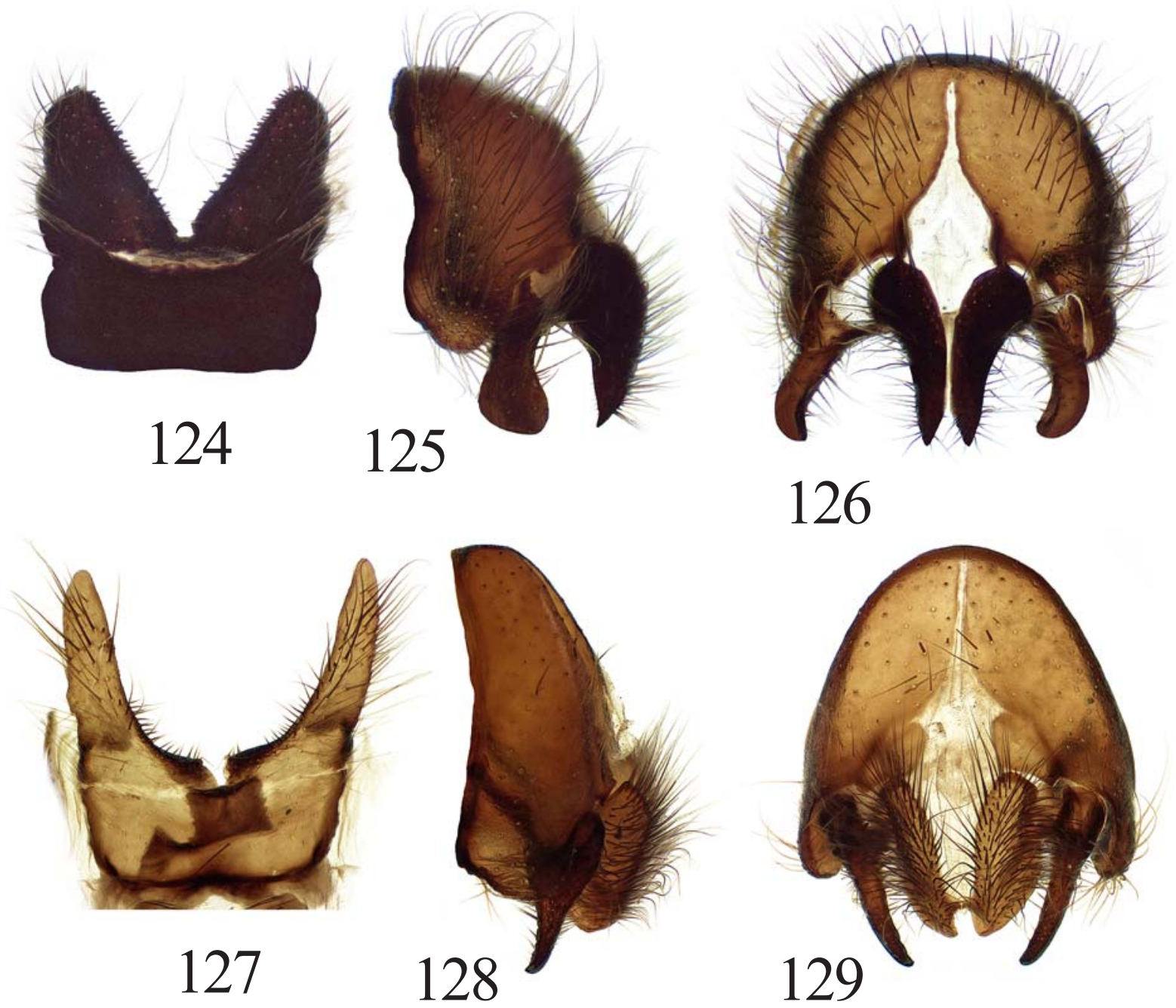

Figs 124-129. Scathophaga varipes (Holmgren) (124-126) and S. yakutica Ozerov (127-129): 124, 127 — male sternite 5; 125, 128 epandrium, cercal plate and surstyli, lateral view; 126, 129 - same, dorsal view. 127-129 - after Ozerov, 2017, figs 1-3.

Pис. 124-129. Scathophaga varipes (Holmgren) (124-126) и S. yakutica Ozerov (127-129): 124, 127 — стернит 5 самца; 125, 128 эпандрий, церки и сурстили, сбоку; 126, 129 - то же, сверху. 127-129 - по Ozerov, 2017, figs 1-3.

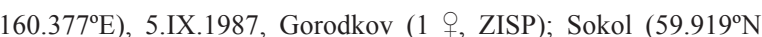
150.752 ${ }^{\circ}$ ), 25.VIII.1966, Gorodkov (4 $0^{7} 0^{7}, 3$ 우, ZISP); Moscow and Moscow Oblast: Bittsa $\left(55.641^{\circ} \mathrm{N} 37.570^{\circ} \mathrm{E}\right), 24$ 31.VII.1936, B. Rohdendorf (5 $0^{7} \sigma^{7}, 1$, ZMUM); Dmitrov env. $\left(56.316^{\circ} \mathrm{N} 37.725^{\circ} \mathrm{E}\right), 8-15 . I X .2006,19 . V ., 21 . V I ., \quad 8$ and 10. VII., and 2.IX.2007, 18.V.2010, N. Vikhrev (13 $\sigma^{7} \sigma^{7}, 4$ 우, ZMUM); Golitsyno $\left(55.649^{\circ} \mathrm{N} 37.011^{\circ} \mathrm{E}\right), 21 . \mathrm{V} .1977,21 . \mathrm{V} .1978,31 . \mathrm{V}$, and 4-7.IX.1981, 5.VI.1982, A. Shatalkin (4 $0^{7} \mathrm{O}^{7}, 4$ oᄋ, ZMUM); Izmaylovo $\left(55.800^{\circ} \mathrm{N} 37.832^{\circ} \mathrm{E}\right)$, 18.VI.1983, A.L. Ozerov (1 O', ZMUM); Zvenigorod env $\left(55.700^{\circ} \mathrm{N} 36.722^{\circ} \mathrm{E}\right)$, 24.V.1981, A.L. Ozerov (4 $\bigcirc^{7} \sigma^{7}, 1$, ZMUM); Murmansk Oblast: Aleksandrovsk [= Polyarnyy $]\left(69.198^{\circ} \mathrm{N} 33.456^{\circ} \mathrm{E}\right)$, IX.1923, Fridolin (1 ㅇ, ZISP); same place, 24-25.VIII.1923, V. Kuznetsov (2 $0^{7} \sigma^{7}, 1$, , ZISP); Lake Vudyavr Basin (ca. $67.646^{\circ} \mathrm{N} 33.644^{\circ} \mathrm{E}$ ), 18 and $23-$ 24.VI.1930, Fridolin ( $3 \mathrm{O}^{7} \mathrm{O}^{7}, 1$, 9 , ZISP); Dal'nie Zelentsy $\left(69.117^{\circ} \mathrm{N}\right.$ $\left.36.062^{\circ} \mathrm{E}\right), 6$ and 10.VIII.1981, 2.IX.1984, Gorodkov (7 $\mathrm{O}^{7} \mathrm{O}^{7}, 1$, ZISP); Khibiny Station $\left(67.673^{\circ} \mathrm{N} 33.212^{\circ} \mathrm{E}\right)$, 4.IX.1928, Cheburova (1 9, ZISP); Kirovsk env. $\left(67.608^{\circ} \mathrm{N} 33.661^{\circ} \mathrm{E}\right), 6$ and 9.VII.1975, Zinov'ev (1 $0^{7}, 1$ ㅇ, ZISP); Kovdor $\left(67.563^{\circ} \mathrm{N} 30.477^{\circ} \mathrm{E}\right)$, 21.VIII.1995, Gorodkov (7 $\bigcirc^{7} 0^{7}, 4$ 오, ZISP); Krasnoshchel'e $\left(67.351^{\circ} \mathrm{N} 37.045^{\circ} \mathrm{E}\right), 18$. VIII.1981, Gorodkov (1 $0^{7}, 2$ 오, ZISP); Sonostrov $\left(66.172^{\circ} \mathrm{N} 34.233^{\circ} \mathrm{E}\right)$, 11.IX.19841, Gorodkov (1 $\sigma^{7}$,
ZISP); Sosnovka $\left(66.5073^{\circ} \mathrm{N} 40.583^{\circ} \mathrm{E}\right), 23 . \mathrm{VIII} .1981$, Gorodkov $\left(10 \odot^{\prime \top} \bigcirc^{\top}, 2\right.$ 우, ZISP); Tumannyy $\left(68.883^{\circ} \mathrm{N} 35.693^{\circ} \mathrm{E}\right), 4$.VIII.1981, Gorodkov (3 $\sigma^{7} \sigma^{7}, 1$ \% , ZISP); Varzuga $\left(66.394^{\circ} \mathrm{N} 36.596^{\circ} \mathrm{E}\right)$, 16.VIII.1995, Gorodkov (3 $\sigma^{7} \sigma^{7}, 1$, , ZISP); Novgorod Oblast: Novgorod env. $\left(58.486^{\circ} \mathrm{N} 31.284^{\circ} \mathrm{E}\right), 1 . X .1978$, Gorodkov (1 $\mathrm{O}^{7}, 2$ 우, ZISP); Ramen'e $\left(58.374^{\circ} \mathrm{N} 33.430^{\circ} \mathrm{E}\right)$, Okulovka env., 4.VI.1988, Gorodkov (4 $0^{7} \sigma^{7}, 1$ ㅇ, ZISP); Novosibirsk Oblast: Koynikha $\left(54.568^{\circ} \mathrm{N} 83.252^{\circ} \mathrm{E}\right), 13$.VIII.1977, Gorodkov $\left(2 \mathrm{O}^{7} \mathrm{O}^{7}\right.$, 1 , ZISP); Primorsky Krai: Terney env. $\left(45.043^{\circ} \mathrm{N} 136.634^{\circ} \mathrm{E}\right)$, 8.X.2016, M. Sergeev (1 +, ZMUM); Ryazan' Oblast: Kasimov env. $\left(54.969^{\circ} \mathrm{N} 41.327^{\circ} \mathrm{E}\right), 21-24 . V I I .2013$, N. Vikhrev (1 $\sigma^{7}$, ZMUM); Sakhalin Oblast: Kunashir I., Kurilsky Nature Reserve, cordon Andreevsky $\left(43.54^{\circ} \mathrm{N} 145.37^{\circ} \mathrm{E}\right), 6-8$.VII.2014, I.A. Gomyranov (1 $\sigma^{7}$, ZMUM); Kunashir I., Sernovodsk $\left(43.912^{\circ} \mathrm{N}\right.$ $\left.145.641^{\circ} \mathrm{E}\right), 8$. VI.1968, V. Richter (10 $0^{7} 0^{\top}, 8$ 우, ZISP); Kunashir I., Yuzhno-Kuril'sk $\left(44.029^{\circ} \mathrm{N} 145.859^{\circ} \mathrm{E}\right), 16 . V I .1968$, V. Richter, (1 $\bigcirc^{7}$, ZISP); same place, 5.VII.1979, A. Zinov'ev (1 $\sigma^{7}$, ZISP); Paramushir I., Severo-Kuril'sk $\left(50.679^{\circ} \mathrm{N} 156.132^{\circ} \mathrm{E}\right)$, 16.VIII.1978, A. Zinov'ev (1 $\bigcirc^{7}$, ZISP); Shikotan I., Malokuril'sk $\left(43.869^{\circ} \mathrm{N}\right.$ $\left.146.829^{\circ} \mathrm{E}\right)$, 24.IX.1968, Gorodkov (28 $\sigma^{7} 0^{7}, 8$ 우, ZISP); Smolensk Oblast: "Smolenskoe poozer'e" National Park $\left(55.511^{\circ} \mathrm{N}\right.$ $\left.31.839^{\circ} \mathrm{E}\right), 29,30$.VII. and 9.VIII.1991, Zlobin ( $20^{7} 0^{7}, 1$ \%, ZISP); 
Tyumen' Oblast: "Elizarovskoe, Tobol. gub." (=Elizarovo: $\left.61.395^{\circ} \mathrm{N} 68.295^{\circ} \mathrm{E}\right), 7 . V I .1907$, M. Lagin (1 ${ }^{7}$, ZMUM); $75 \mathrm{~km}$ WSW of Samburg (ca. $67.035^{\circ} \mathrm{N} 76.541^{\circ} \mathrm{E}$ ), 6-9.VIII.1976, Gorodkov $\left(90^{7} \mathrm{O}^{7}, 8\right.$ 오, ZISP); Labytnangi $\left(66.657^{\circ} \mathrm{N} 66.391^{\circ} \mathrm{E}\right), 30$.VI. 8-21.VII.1973, 28.VII.1981, V. Sychevskaya, G.A. Veselkin (11 $o^{7} O^{7}, 4$ 우, ZMUM); Cape Kamennyy $\left(68.495^{\circ} \mathrm{N} 73.582^{\circ} \mathrm{E}\right)$, 9.VIII.1967, Gorodkov (3 $\mathrm{O}^{7} \mathrm{O}^{7}, 3$ 우, ZISP); Neroyka (ca. $64.57^{\circ} \mathrm{N}$ $\left.59.67^{\circ} \mathrm{E}\right), 650 \mathrm{~m}, 6 . \mathrm{IX} .1990$, Malozemov (1 $\sigma^{7}$, ZISP); same place, $450 \mathrm{~m}, 29$.VI.1990, Malozemov (1 $\sigma^{7}$, ZISP); Salekhard $\left(66.53^{\circ} \mathrm{N}\right.$ $66.613^{\circ} \mathrm{E}$ ), 3.VIII.1961, Gorodkov (4 $0^{7} \sigma^{7}, 2$ 우, ZISP); same place, 4-5.VIII.1976, Gorodkov (3 $\sigma^{7} \sigma^{7}, 2$ 우, ZISP); Saranpaul' $\left(64.257^{\circ} \mathrm{N} 60.917^{\circ} \mathrm{E}\right), 23 . V I .1989$, Malozemov (2 $0^{7} \mathrm{O}^{7}$, ZISP); Tarko-Sale $\left(64.914^{\circ} \mathrm{N} 77.766^{\circ} \mathrm{E}\right), 1$ and 2.IX.1982, Gorodkov $(4$ $O^{7} O^{7}, 5$ 우, ZISP); Vologda Oblast: Vologda env. $\left(59.207^{\circ} \mathrm{N}\right.$ $\left.39.904^{\circ} \mathrm{E}\right)$, 8.IX.1981, Gorodkov (1 9 , ZISP); Yakutia: Aykha $\left(65.944^{\circ} \mathrm{N} 111.495^{\circ} \mathrm{E}\right), 19 . \mathrm{VIII} .1988$, Gorodkov $\left(1 \mathrm{O}^{7}, 1\right.$ ㅇ, ZISP); Batagay $\left(67.653^{\circ} \mathrm{N} 134.635^{\circ} \mathrm{E}\right), 31 . \mathrm{VII} .2008$, A. Ovchinnikov (1 , ZISP); Bestyakh $\left(65.281^{\circ} \mathrm{N} 124.120^{\circ} \mathrm{E}\right), 14 . \mathrm{VI} .1912$, Naumov (1 ZISP); Khaptagay $\left(61.787^{\circ} \mathrm{N} 129.795^{\circ} \mathrm{E}\right), 30 . \mathrm{VI} .1974$, Nartshuk (1 $\sigma^{7}$, ZISP); left bank of the River Yana opposite of Verkhoyansk $\left(67.55^{\circ} \mathrm{N} 133.359^{\circ} \mathrm{E}\right), 21$ and 23.VII.1974, Nartshuk $\left(5 \mathrm{O}^{7} \mathrm{O}^{7}, 3\right.$ O ZISP); Lensk $\left(60.727^{\circ} \mathrm{N} 114.943^{\circ} \mathrm{E}\right), 12$ and 14.IX.1987, Gorodkov (1 $0^{7}, 1$, , ZISP); Mikhaylovka, $60 \mathrm{~km}$ NE of Amga (61.214 $\mathrm{N}$ $132.681^{\circ} \mathrm{E}$ ), 15.VIII.1984, Averinskiy (5 $\sigma^{7} \sigma^{7}$, ZISP); Mirnyy $\left(62.540^{\circ} \mathrm{N} 113.962^{\circ} \mathrm{E}\right), 22$. VIII.1988, Gorodkov (1 $0^{7}$, ZISP); Neryuktyayinsk-1 $\left(60.258^{\circ} \mathrm{N} 119.673^{\circ} \mathrm{E}\right), 11$.VII. 2008, A. Ovchinni$\operatorname{kov}\left(2 \mathrm{O}^{\top} \sigma^{7}, 1\right.$ \%, ZISP); Nyurba $\left(63.285^{\circ} \mathrm{N} 118.331^{\circ} \mathrm{E}\right), 18 . \mathrm{VI} .1987$, Zlobin (3 $0^{7} 0^{7}, 1$ ․ ZISP); Oktemtsy env. $\left(61.672^{\circ} \mathrm{N} 129.417^{\circ} \mathrm{E}\right)$, 18.VII.1977, Bagachanova (1 +. ZISP); Olekminsk $\left(60.370^{\circ} \mathrm{N}\right.$

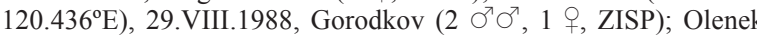
$\left(68.504^{\circ} \mathrm{N} 112.457^{\circ} \mathrm{E}\right), 9 . \mathrm{VIII} .1973$, Gorodkov $\left(10 \mathrm{O}^{7} \mathrm{O}^{7}, 2\right.$ 오, ZISP); Oleneksky Bay, Ystannakh-Khocho $\left(72.58^{\circ} \mathrm{N} 121.42^{\circ} \mathrm{E}\right)$ 20.VIII.2010, A. Yadrenkin (1 $\bigcirc^{7}$, ZMUM); Pokrovsk $\left(61.467^{\circ} \mathrm{N}\right.$ $\left.129.101^{\circ} \mathrm{E}\right)$, 21.VII.1986, Makarkin (2 $\mathrm{O}^{\mathrm{T}} \mathrm{O}^{\mathrm{T}}$, IBSS); River Yana, Stolby env. (67.531 $\left.{ }^{\circ} \mathrm{N} 134.087^{\circ} \mathrm{E}\right), 29 . \mathrm{VII}$. 2008, A. Ovchinnikov $\left(6 \mathrm{O}^{7} \mathrm{O}^{7}, 1\right.$ ㅇ, ZISP); Saskylakh env. $\left(71.966^{\circ} \mathrm{N} 114.094^{\circ} \mathrm{E}\right)$ 9.VIII.1988, Gorodkov (11 $\sigma^{7} \sigma^{7}, 1$ ㅇ, ZISP); Srednekolymsk $\left(67.460^{\circ} \mathrm{N} 153.710^{\circ} \mathrm{E}\right)$, 16.VIII.1974, Gorodkov $\left(6 \mathrm{O}^{7} \mathrm{O}^{7}, 3\right.$ 우, ZISP); Tiksi env. $\left(71.635^{\circ} \mathrm{N} 128.857^{\circ} \mathrm{E}\right), 30 . \mathrm{VII} .1990$, Kaspyaryan (2 우, ZISP); Tolon $\left(59.458^{\circ} \mathrm{N} 111.539^{\circ} \mathrm{E}\right), 14$.VII.1987, Zlobin (1 $\mathrm{O}^{7}$, ZISP); Tommot $\left(58.963^{\circ} \mathrm{N} 126.282^{\circ} \mathrm{E}\right), 23$.VIII.1969, Sychevskaya (1 $\mathrm{O}$, ZISP); Udachnyy $\left(66.405^{\circ} \mathrm{N} 112.2994^{\circ} \mathrm{E}\right)$, 21.VIII.1988, Gorodkov (1 $\bigcirc^{7}$, ZISP); Verkhoyansk $\left(67.548^{\circ} \mathrm{N}\right.$ $\left.133.396^{\circ} \mathrm{E}\right), 3$.VIII.1969, Sychevskaya (3 $0^{7} 0^{7}, 1$. , ZISP); Vilyuysk env. $\left(63.750^{\circ} \mathrm{N} 121.637^{\circ} \mathrm{E}\right), 24 . \mathrm{VIII} .1988$, Gorodkov (1 , ZISP); Yakutsk env. (62.034 $\left.\mathrm{N} 129.618^{\circ} \mathrm{E}\right)$, 3.VIII.2008, A. Ovchinnikov (1 $0^{7}$, ZISP); Zhigansk $\left(66.766^{\circ} \mathrm{N} 123.372^{\circ} \mathrm{E}\right)$, 715.VIII.1973, Gorodkov (7 O $^{7} 0^{7}, 5$ 웅, ZISP); Zabaikalsky Krai: Chita $\left(52.065^{\circ} \mathrm{N} 113.444^{\circ} \mathrm{E}\right), 10 . \mathrm{IX} .1974$, Gorodkov (1 $\circ$, ZISP); Ingoda $\left(51.851^{\circ} \mathrm{N} 113.097^{\circ} \mathrm{E}\right), 7 . \mathrm{VII} .1971, \mathrm{~V}$. Richter $\left(2 \mathrm{O}^{7} \mathrm{O}^{7}\right.$, ZISP); Kyra $\left(49.581^{\circ} \mathrm{N} 111.974^{\circ} \mathrm{E}\right), 17 . \mathrm{VI} .1975$, V. Richter $\left(1 \mathrm{O}^{\prime \prime}\right.$ ZISP); Olovyannaya $\left(50.994^{\circ} \mathrm{N} 115.561^{\circ} \mathrm{E}\right), 9 . \mathrm{VII} .1971$, V. Richter (1 $0^{7}$, ZISP). See also Ozerov \& Krivosheina [2014: 218, as $S$. suilla] and Ozerov \& Barkalov [2014: 564, as S. suilla].

DESCRIPTION. Male. Female. Body-length 3.8$7.7 \mathrm{~mm}$.

Head. Frontal vitta yellow or reddish-yellow, matt; fronto-orbital plate blackish, greyish dusted. Face, parafacial and gena yellow, with delicate whitish reflection. Postcranium black in upper third or half and yellow in lower part. 3 orbital and 3-4 frontal setae present. Antenna reddish-yellow. Postpedicel approximately twice as long as wide. Arista pubescent or plumose, the longest hairs approximately equal or more than $1 / 2$ width of postpedicel. Palpus yellow.

Thorax greyish dusted. Scutum black completely, only postpronotal lobe usually yellowish; pleural sclerites yellowish in ground color, but anatergite, katatergite and mediotergite always black. Acrostichals setulose in two rows, but prescutellar pair stronger than other acrostichals, dorsocentrals $2+3$, intra-alars $1+2$, supraalars $1+2$, postpronotals 2 . Katepisternum posteriorly with yellow hairs and setulae and, as a rule, without black setulae. Anepimeron bare. Scutellum black, greyish dusted, with a pair of strong basal scutellar and a pair of strong apical scutellar setae.

Legs yellow. Fore femur covered with hairs, but without conspicuous setae in male, with a row of thin dorsal setae in female. Fore tibia with 2 dorsal, 0-1 posterior, 1 preapical dorsal, and 1 posterior apical setae. Mid femur with 1 preapical seta in male and usually with a row of anterodorsal setae in female, also with 1 preapical posterior and 1 preapical posterodorsal setae in both sexes. Mid tibia with 1-2 posterodorsal, 1 anterodorsal setae and a ring of apicals. Hind femur without conspicuous setae in male and with a row of anterodorsal setae in female. Hind tibia with $2-$ 3 anterodorsal, 2-3 posterodorsal, 1 preapical dorsal, 1 preapical anterodorsal, 1 apical anteroventral setae.

Wing tinged with brownish; crossveins r-m and dmcu darkened.

Abdomen from yellowish to dark brownish, delicately greyish dusted, covered with not long hairs; female tergites with black marginal setulae. Female tergite 7 shiny. Male sternite 4 approximately 2 times as long as wide (Fig. 37); sternite 5 with a pair of narrow median processes (Fig. 112). Epandrium, cercal plate and surstyli as in Figs 113, 114.

DISTRIBUTION. Russia: throughout midland, north to southern tundra. - Europe (widespread), China, Japan, Kazakhstan, Mongolia, Syria [Šifner, 2008: 172], North America; a widespread and common species, but more abundant in the forest zone.

Scathophaga stercoraria (Linnaeus, 1758) Figs 2, 38, 115-117.

stercoraria Linnaeus, 1758: 599 (Musca). Type-locality: not given (?Sweden)

merdaria Fabricius, 1794: 344 (Musca). Type-locality: "Habitat Kiliae" [= Kiel, Germany].

erythrostoma Holmgren, 1883: 176 (Scatomyza). Type-locality: "Matotschkin Scharr" [Matochkin Schar, Novaya Zemya] (Russia); [synonymized by Šifner, 2008: 170].

asticha Szilády, 1926: 594 (Scatophaga, as ab. of merdaria).

Type-localities: "Budapest und Pusztapó, aus Tunis und aus dem Kaukasus" (Hungary, Tunisia, Russia).

asticha Szilády, 1926: 594 (Scatophaga, as ab. of stercorar-

ia). Type-locality: "Topánfalva" [ = Cîmpeni] Roumania). disticha Szilády, 1926: 594 (Scatophaga, as ab. of stercorar-

ia). Type-localities: "verschiedenen Gegenden Ungarns und aus Tomsk, Sibirien" (Hungary and Russia).

nigricans Szilády, 1926: 595 (Scatophaga, as var. of stercoraria). Type-locality: "Island" (Iceland).

polysticha Szilády, 1926: 594 (Scatophaga, as ab. of merda-

ria). Type-localities: "Ungarn und Tunis" (Hungary and Tunisia). alpestre Sack, 1937: 58 (Scopeuma, as var. of stercoraria). Type-locality; not given.

REMARKS. This species was registered in Russia by many authors (e.g., Hendel [1930: 2], Gorodkov [1970: 451, 1986: 33], Sychevskaya [1972: 150], Veselkin [1985: 75], Verves et al. [1990: 141], Ovchin- 
nikov [2004: 422], Ozerov [2009: 379], Ozerov \& Krivosheina [2014: 218], Ozerov \& Barkalov [2014: 564], Bagachanova et al. [2016: 782, 783], Ovchinnikov \& Makarova [2016: 217], MacGowan et al. [2021: 17]).

Gorodkov published a map with the distribution area of this species on the territory of the former USSR and separately on its European part [Gorodkov, 1981: map 121], but without names of the points.

MATERIAL EXAMINED. About 4000 specimens taken from the majority of Regions of Russia were examined in the collections of ZMUM and ZISP, including pubished material [Ozerov \& Krivosheina, 2014: 218, 219; Ozerov \& Barkalov, 2014: 564].

DESCRIPTION. Male. Female (Fig. 2). Body-length 4.6-9.8 $\mathrm{mm}$.

Color, length, and thickness of hairs on the body vary greatly, with males usually paler than females. As a rule, thorax, abdomen, and legs of male with dense, golden hairs, and females with less dense, greenishyellow or black hairs. Chaetotaxy better seen in females (Fig. 00), and black setae in males often hidden among dense hairs. Pollination on thorax, abdomen, and legs varies from golden-yellow to greenish-grey in both sexes.

Head. Frontal vitta reddish-yellow, with delicate whitish reflection; fronto-orbital plate black, greyish dusted. Face, parafacial and gena yellow, with delicate whitish or golden reflection. Postcranium black. 3 orbital and 3 6 frontal setae present. Antenna black. Postpedicel approximately 2 times as long as wide. Arista pubescent or plumose, the longest hairs approximately equal or more than $1 / 2$ width of postpedicel. Palpus yellow.

Thorax black, densely greyish dusted, with dark stripes and fuzzy spots on scutum and scutellum. Acrostichals setulose in two rows, but prescutellar pair stronger than the other acrostichals, dorsocentrals $2+3$, intraalars $1+2$, supra-alars $1+2$, postpronotals 2 . Anepimeron covered with hairs. Scutellum black, greyish dusted, with a pair of strong basal scutellar and a pair of strong apical scutellar setae, also with a pair of discal setulae.

Legs densely greyish dusted. All coxae black; all femora black, except apex; all tibiae and tarsi yellow. Fore femur covered with hairs, with a row of thin ventral setae in both sexes, additionally with a row of dorsal setae in female. Fore tibia with 2-3 dorsal, 1-2 posterior, 1 preapical dorsal, and 1 posterior apical setae. Mid femur with a row of anterodorsal setae, also with 1 preapical posterior and 1 preapical posterodorsal setae in both sexes. Mid tibia with 3-4 posterodorsal, 3 anterodorsal, 1-2 anteroventral, 1 posteroventral setae and a ring of apicals. Hind femur with 2-5 anterodorsal setae in apical third or half in male and a row of anterodorsal setae in female. Hind tibia with 3-4 anterodorsal, 3-4 posterodorsal, 1-2 anterior, 1 preapical dorsal, 1 preapical anterodorsal, 1 apical anterior and 1 apical anteroventral setae in both sexes, additionally with 2-3 anteroventral setae in female.

Wing tinged with brownish; crossvein r-m slightly to distinctly darkened, crossveins $\mathrm{r}-\mathrm{m}$ as a rule not darkened.
Abdomen black, densely greyish dusted, covered with dense hairs in male; in female tergites with black marginal setulae. Male sternite 4 approximately 2 times as long as wide (Fig. 38); sternite 5 with short lateral lobes and a pair of short median processes (Fig. 115). Epandrium, cercal plate and surstyli as in Figs 116, 117.

Description and figures of female ovipositor are given by Ovchinnikov [2009: 625, 626].

DISTRIBUTION. Throughout Holarctic region; Oriental region: China, India, Nepal [Vockeroth, 1977: 438], Vietnam [Ozerov, Krivosheina, 2011: 3]; Haiti [Vockeroth, 2010: 1269]; the commonest species.

\section{Scathophaga taeniopa (Rondani, 1866) Figs 4, 39, 118-120.}

taeniopa Rondani, 1866: 27; 1867: 111 (Scatophaga). Typelocality: "in collibus agri parmensis" (vicinity of Parma, Italy).

ordinata Becker, 1894: 168 (Scatophaga). Type-locality: "St. Moritz" (Switzerland).

REMARK. This species was recorded in Russia from Sayan Mountains and Kuban [Gorodkov, 1970: 451], Komi and Caucasus [Gorodkov, 1986: 34], Yakutia [Veselkin, 1985: 75; Bagachanova et al., 2016: 783], Far East [Ozerov, Krivosheina, 2014: 219].

MATERIAL EXAMINED. Altai: "Rodonovy istochnik", 2412 m, Lake Muzdy-Bulak (49.46 $\left.\mathrm{N} 88.05^{\circ} \mathrm{E}\right)$, 5.VII.2008, A. Barkalov (2 $\sigma^{\top} \sigma^{\top}, 2$ OO, ISEA); Bol'shoy Yaloman $\left(50.489^{\circ} \mathrm{N} 86.416^{\circ} \mathrm{E}\right)$, 9.VII.1967, R. Kamenskaya (1 $\bigcirc^{\top}$, ISEA); Chuysky ridge, 2185$2600 \mathrm{~m}\left(49.65^{\circ} \mathrm{N}, 88.22^{\circ} \mathrm{E}\right), 12-13$.VII.2009, A. Barkalov (1 ア 7 ㅇ, ISEA); Kosh-Agach, plateau Ukok, 2400 m, Lake MuzdyBulak, env. $\left(49.26^{\circ} \mathrm{N}, 87.65^{\circ} \mathrm{E}\right), 10 . \mathrm{VII} .2008$, A. Barkalov (1 + , ISEA); Onguday env. (50.77 $\left.\mathrm{N} 86.09^{\circ} \mathrm{E}\right), 8-13$.VII.2016, N. Vikhrev (1 $\sigma^{7}$, ZMUM); Tigirek Vill. $\left(51.13^{\circ} \mathrm{N} 83.05^{\circ} \mathrm{E}\right), 550 \mathrm{~m}, 9$ 12.VII.2014, I. Shamshev (1 $q$, ZISP); Amur Oblast: Bol'shoy Never $\left(53.978^{\circ} \mathrm{N} 124.150^{\circ} \mathrm{E}\right)$, 8.IX.1974, Gorodkov (3 $\sigma^{\top} \sigma^{7}, 4$ 우, ZISP); Buryatia: Khamney $\left(50.402^{\circ} \mathrm{N} 103.868^{\circ} \mathrm{E}\right), 28 . \mathrm{VI} .1971$, V. Richter (1 $\sigma^{T}$, ZISP); Lake Baykal, Pokoyniki env. $\left(53.713^{\circ} \mathrm{N}\right.$ $\left.109.049^{\circ} \mathrm{E}\right), 21$.VII.1962, Gorodkov (2 $\sigma^{7} \sigma^{7}, 1$ \%, ZISP); Mondy env. $\left(51.675^{\circ} \mathrm{N} 100.992^{\circ} \mathrm{E}\right), 17-18$.VIII.1962, 18-19.VII., 24.VII. and 4.VIII.1965, Gorodkov (9 $\sigma^{7} \sigma^{7}, 8$ कo , ZISP); Chelyabinsk Oblast: Kyshtym $\left(55.715^{\circ} \mathrm{N} 60.551^{\circ} \mathrm{E}\right), 23$.VII.-2.VIII.1929, Burakova ( 1 \%, ZISP); Irkutsk Oblast: Listvyanka $\left(51.863^{\circ} \mathrm{N} 104.866^{\circ} \mathrm{E}\right)$, 24.VI. and 6.VII.1965, Negrobov (2 $\sigma^{7} \sigma^{7}$, ZISP); Irkutsk: $\left(52.332^{\circ} \mathrm{N}\right.$ $\left.104.249^{\circ} \mathrm{E}\right)$, 5.VII.1912, Kornakova (1 $\sigma^{\gamma}$, ZISP); Kirov Oblast: Kirov env. $\left(58.596^{\circ} \mathrm{N} 49.663^{\circ} \mathrm{E}\right), 28 . \mathrm{VIII} .1962$, Kalinkova (1 + , ZISP); Komi: Shchel'yayur $\left(65.33^{\circ} \mathrm{N} 53.43^{\circ} \mathrm{E}\right), 12 . \mathrm{VIII} .1978$, Gorodkov ( $4 \sigma^{\top} \sigma^{\top}, 1$, 1 , ZISP); Krasnodar Krai: River Tsetse (ca. $44.614^{\circ} \mathrm{N} 39.548^{\circ} \mathrm{E}$ ), 13 and 16.VI.1903, Filipchenko ( $1 \sigma^{7}, 1$, ZISP); Teberdinskiy Nature Reserve, $\left(43.443^{\circ} \mathrm{N} 41.738^{\circ} \mathrm{E}\right)$, 3.IX.1965, Gorodkov (2 $\sigma^{7} \sigma^{7}, 2$ 우, ZISP); Teberdinsky Nature Reserve, "Severnyy priyut" ( $\left.43.252^{\circ} \mathrm{N} 41.829^{\circ} \mathrm{E}\right)$, 25.VIII.1980, Tanasiychuk (1 $\sigma^{7}, 1$, ZISP); Krasnoyarsk Krai: Buyba $\left(52.815^{\circ} \mathrm{N} 93.294^{\circ} \mathrm{E}\right)$, 3.VIII.1963, Grunin (3 $\sigma^{\top} \sigma^{7}$, ZISP); Lake Il'chir env. (51.973 $\left.{ }^{\circ} \mathrm{N} 100.993^{\circ} \mathrm{E}\right), 2$.VIII.1965, Gorodkov $\left(20 \mathrm{O}^{7} \sigma^{7}\right.$, 15 우, ZISP); Moscow and Moscow Oblast: Kosino $\left(55.721^{\circ} \mathrm{N}\right.$ $\left.37.847^{\circ} \mathrm{E}\right)$, 8.VIII.1912, E. Smirnov (1 9 , ZMUM); North Ossetia - Alania: Tsey $\left(43.793^{\circ} \mathrm{N} 43.922^{\circ} \mathrm{E}\right), 16 . \mathrm{IX} .1989$, A. Shatalkin (1 ${ }^{7}, 1$ o, ZMUM); Omsk Oblast: Omsk $\left(54.969^{\circ} \mathrm{N} 73.360^{\circ} \mathrm{E}\right)$, 26.VI.2008, O. Kosterin (1 9, ZMUM); Primorsky Krai: Gornotaezhnoe $\left(43.697^{\circ} \mathrm{N} 132.156^{\circ} \mathrm{E}\right), 17 . \mathrm{VI} .1993$, M. Mikhaylovskaya $\left(3 \sigma^{7} \sigma^{\top}, 4\right.$ \% 4 , ZISP); Primorsky $\left(43.097^{\circ} \mathrm{N} 131.587^{\circ} \mathrm{E}\right)$, 26.VIII.1980, Kireychuk (1 9 , ZISP); Smolensk Oblast: "Smolenskoe poozer'e" National Park $\left(55.511^{\circ} \mathrm{N} 31.839^{\circ} \mathrm{E}\right), 12 . \mathrm{VIII} .1991$, Zlobin (1 9 , ZISP); Yakutia: $20 \mathrm{~km}$ above of the mouth of the River Biryuk $\left(60.434^{\circ} \mathrm{N} 119.497^{\circ} \mathrm{E}\right), 15$.VII. 2008, A. Ovchinni-

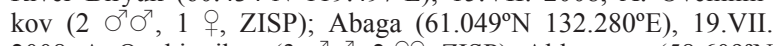
2008, A. Ovchinnikov (3 $\sigma^{\top} \sigma^{\top}, 2$, 2 , ZISP); Aldan env. $\left(58.609^{\circ} \mathrm{N}\right.$ 
125.373 $\left.{ }^{\circ} \mathrm{E}\right)$, 30.VIII.1974, Gorodkov (21 $\sigma^{7} \sigma^{7}, 13$ o $1+$, ZISP); same place, 3.VIII.1977, G. Veselkin (15 $\sigma^{7} \sigma^{7}, 1$ \%, ZMUM); Arka $\left(60.077^{\circ} \mathrm{N} 142.335^{\circ} \mathrm{E}\right), 26 . V I I I .1987$, Gorodkov (1 ${ }^{7}$, ZISP); Khaptagay $\left(61.785^{\circ} \mathrm{N} 129.797^{\circ} \mathrm{E}\right), 23$. VI.-4.VII.1974, L. Zimina, R. Kamenskaya $\left(12 \sigma^{7} \sigma^{\top}, 9\right.$ 우, ZMUM); Khaptagay $\left(61.787^{\circ} \mathrm{N} 129.795^{\circ} \mathrm{E}\right)$ 29.VI.1974, Nartshuk (4 $\sigma^{\top} \sigma^{\top}, 6$ 6 , Z ZISP); Kutana $\left(62.713^{\circ} \mathrm{N}\right.$

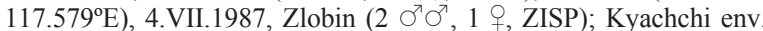
$\left(60.348^{\circ} \mathrm{N} 120.084^{\circ} \mathrm{E}\right), 20$. VII.2008, A. Ovchinnikov (1 $\sigma^{\top}, 2$ 우, ZISP); left bank of the River Yana opposite of Verkhoyansk $\left(67.55^{\circ} \mathrm{N}\right.$ 133.359 $\left.9^{\circ} \mathrm{E}\right), 23$. VII.1974, Nartshuk (1 $\sigma^{\top}$, ZISP); Lensk $\left(60.727^{\circ} \mathrm{N}\right.$

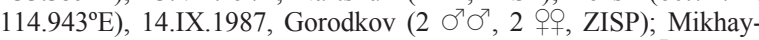
lovka $\left(61.214^{\circ} \mathrm{N} 132.681^{\circ} \mathrm{E}\right), 1 . \mathrm{VIII} .1987, \mathrm{~V}$. Zlobin (1 $\mathrm{O}^{7}$, ZISP); same place, 2 and 4.VIII.1984, 4.VIII.1986, 8.VIII.1987, Bagachanova, Averinskiy (6 우, ZISP); Neryuktyayinsk-1 $\left(60.258^{\circ} \mathrm{N}\right.$ 119.673 $\left.{ }^{\circ} \mathrm{E}\right), 11,17$ and 18.VII. 2008, A. Ovchinnikov (8 $\sigma^{\top} \sigma^{\top}, 11$ 우, ZISP); Oktemtsy env. (61.672 $\left.\mathrm{N} 129.417^{\circ} \mathrm{E}\right), 4-7 . \mathrm{VII} .2008$, A Ovchinnikov ( 6 우, 1 ㅇ, ZISP); Olekminsk $\left(60.370^{\circ} \mathrm{N} 120.436^{\circ} \mathrm{E}\right)$ 6.VIII.1974, Nartshuk (1 $\sigma^{7}$, ZISP); same pace, 29-31.VIII.1988, Gorodkov ( $3 \bigcirc^{\top} \sigma^{\top}, 8$, 8 , ZISP); River Biryuk, 4 km below the mouth of the River Melichan (ca. $60.434^{\circ} \mathrm{N} 119.495^{\circ} \mathrm{E}$ ), 14.VII.2008, A. Ovchinnikov (2 $\sigma^{7} \sigma^{7}, 1+$, ZISP); the mouth of the River Olekmy

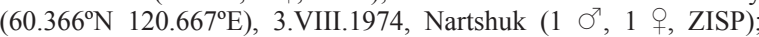
Tommot $\left(58.963^{\circ} \mathrm{N} 126.282^{\circ} \mathrm{E}\right), 23$.VIII.1969, Sychevskaya $\left(1 \sigma^{\top}, 2\right.$ 웅, ZISP); Tyungyulyu (62.201 $\left.{ }^{\circ} \mathrm{N} 130.676^{\circ} \mathrm{E}\right), 21$. VIII.1989, Vinokurova (3 $\sigma^{7} \sigma^{7}, 1$, ZISP); same place, 30.VIII.2003, Dedyukina $\left(2\right.$ 오, ZISP); Yakutsk $\left(62.034^{\circ} \mathrm{N} 129.618^{\circ} \mathrm{E}\right), 21$. VII.1987, Zlobin $\left(4 \Im^{7} \sigma^{7}, 2\right.$ 우, ZISP); Zhigansk $\left(66.766^{\circ} \mathrm{N} 123.372^{\circ} \mathrm{E}\right)$ 15.VIII.1973, Gorodkov (1 + Z, ZISP); Zabaikalsky Krai: Baley $\left(51.580^{\circ} \mathrm{N} 116.638^{\circ} \mathrm{E}\right), 18 . \mathrm{VII} .1971$, V. Richter (1 , ZISP); Ingoda $\left(51.851^{\circ} \mathrm{N} 113.097^{\circ} \mathrm{E}\right), 7 . V I I .1971, \mathrm{~V}$. Richter (1 + , ZISP); Elizavetino $\left(51.658^{\circ} \mathrm{N} 113.605^{\circ} \mathrm{E}\right), 26$. VIII.1981, V. Zherikhin (1 $\sigma^{7}$ ZMUM). See also Ozerov \& Krivosheina [2014: 219].

DESCRIPTION. Male. Female. Body-length 7.1$9.6 \mathrm{~mm}$.

Head (Fig. 4). Frontal vitta yellow or reddish-yellow, with delicate whitish reflection; fronto-orbital plate blackish, greenish grey dusted. Face, parafacial and gena yellow, with delicate golden reflection. Postcranium black in upper third or half and yellow in lower part. 3 orbital and 4-6 frontal setae present. Antenna reddish-yellow. Postpedicel approximately twice as long as wide. Arista pubescent or plumose, the longest hairs approximately equal or more than $1 / 2$ width of postpedicel. Palpus yellow.

Thorax black in ground colour, greyish dusted, but usually postpronotal lobe yellowish. Acrostichals not differentiated from the other hairs on scutum or setulose in two irregular rows, but prescutellar pair stronger than the other acrostichals, dorsocentrals $2+3$, intra-alars $1+2$, supra-alars $1+2$, postpronotals 2 . Katepisternum posteriorly besides yellow hairs and setulae, as a rule also with several black setulae. Anepimeron bare. Scutellum black, greyish dusted, with a pair of strong basal scutellar and a pair of strong apical scutellar setae.

Legs yellow. Fore femur covered with hairs, but without conspicuous setae. Fore tibia with 2 dorsal, 0-1 posterior, 1 preapical dorsal, and 1 posterior apical setae. Mid femur with 1 preapical seta in male and usually with a row of anterodorsal setae in female, also with 1 preapical posterior and 1 preapical posterodorsal setae in both sexes. Mid tibia with 2 posterodorsal, 1 anterodorsal setae and a ring of apicals. Hind femur with 1-2 preapical anterodorsal setae in male and with a row of anterodorsal setae in female. Hind tibia with 3-4 anterodorsal, 2-3 posterodorsal,
1 preapical dorsal, 1 preapical anterodorsal, 1 apical anteroventral setae.

Wing tinged with brownish; crossveins r-m and dmcu slighty darkened.

Abdomen from yellowish to dark brownish, delicate greyish dusted, covered with not long hairs, female tergites with black marginal setulae. Female tergite 7 shiny. Male sternite 4 approximately 2 times as long as wide (Fig. 39); sternite 5 with a pair of median processes (Fig. 118). Epandrium, cercal plate and surstyli as in Figs 119, 120; surstylus broad in median part.

Photo of female ovipositor was given by Ovchinnikov [2009: 636, fig. 30].

DISTRIBUTION. Russia: throughout midland, north to northern subarctic tundra. - Europe, Kazakhstan, Mongolia, China.

\section{Scathophaga tinctinervis (Becker, 1894)}

Figs 40, 121-123, 137.

tinctinervis Becker, 1894: 178 (Coniosternum). Type-locality: locality not stated.

REMARK. This species was recorded in Russia from Leningrad Oblast [Gorodkov, 1986: 34] and Primorsky Krai [Ozerov, Krivosheina, 2014: 219].

MATERIAL EXAMINED. Amur Oblast: Klimoutsy $\left(51.470^{\circ} \mathrm{N}\right.$ $\left.127.599^{\circ} \mathrm{E}\right), 25 . \mathrm{IX} .1958$, Zinov'ev (1 $\sigma^{\top}$, ZISP); Leningrad Oblast: Gatshina $\left(59.56^{\circ} \mathrm{N} 30.13^{\circ} \mathrm{E}\right), 9 . V .1954$, A. Stackelberg (1 $\left.\bigcirc^{7}, \mathrm{ZISP}\right)$; Moscow Oblast: Golitsyno $\left(55.649^{\circ} \mathrm{N} 37.011^{\circ} \mathrm{E}\right), 17$ and $24 . X .1981$, A. Shatalkin $\left(1 \mathrm{O}^{7}, 1\right.$ \% , ZMUM); Naro-Fominsk $\left(55.357^{\circ} \mathrm{N} 36.736^{\circ} \mathrm{E}\right)$, 1.IV.2008, D. (1 + , ZMUM); Novosibirsk Oblast: Evsino env. $\left(54.53^{\circ} \mathrm{N} 83.38^{\circ} \mathrm{E}\right), 20$. VI.2015, O. Kosterin (1 $\sigma^{7}$, ZMUM); Novosibirsk $\left(54.825^{\circ} \mathrm{N} 83.114^{\circ} \mathrm{E}\right), 27 . \mathrm{IV} .2008$, O. Kosterin (2 $\left.\sigma^{\top} \sigma^{\top}, \mathrm{ZMUM}\right)$. See also Ozerov \& Krivosheina [2014: 219].

ADDITIONAL MATERIAL EXAMINED. Mongolia: $25 \mathrm{~km}$ WSW of Ikhtamir (ca. $47.4937^{\circ} \mathrm{N} 100.9143^{\circ} \mathrm{E}$ ), 30.VIII.1967, Zaitzev ( $8 \bigcirc^{7} \sigma^{7}, 10$ 우, ZISP) [determined by Gorodkov as $S$. grisea Malloch, 1920].

DESCRIPTION. Male. Female. Body-length 5.4$6.8 \mathrm{~mm}$.

Head. Frontal vitta yellow, with delicate whitish reflection; fronto-orbital plate blackish, densely greyish dusted. Face, parafacial and gena yellow, with whitish reflection. Postcranium blackish. 3 orbital and 4-5 frontal setae present. Antenna black; postpedicel about twice as long as wide. Arista bare. Palpus yellow.

Thorax black, densely greyish dusted, with dark stripes and fuzzy spots on scutum and scutellum. Acrostichals not differentiated from the other hairs on scutum, dorsocentrals $2+3$, intra-alars $1+2$ (posterior small), supra-alars $1+2$, postpronotals 2. Anepimeron with several hairs. Scutellum greyish dusted, with a pair of strong basal scutellar and a pair of strong apical scutellar setae.

Legs. All coxae black, greyish dusted. All femora greyish dusted, black, except yellow apical quarter or third. All tibiae and all tarsi yellow. Fore femur covered with whitish hairs, with 3-4 dorsal setae or setulae in apical third. Fore tibia with 2-4 dorsal, 2-4 posterodorsal, 1-2 posterior, 1 preapical dorsal, 1 preapical anterodorsal, and 1 posterior apical setae. 
Mid femur with a row of anterior setae, 1 preapical posterior and 1 preapical posterodorsal setae. Mid tibia with 1-2 anterodorsal, 1-2 posterodorsal, 0-1 anteroventral, 0-1 posterior setae, also with a ring of apicals. Hind femur with a row of anterodorsal setae. Hind tibia with 2-3 posterodorsal, 2-3 anterodorsal, 0-1 anteroventral, $0-1$ posterior setae, 1 preapical dorsal, 1 preapical anterodorsal and 1 apical anteroventral setae.

Wing clear; crossveins r-m and d-m not darkened.

Abdomen black, densely pale grey dusted, covered with short whitish hairs; tergites 2-6 each in both sexes with a row of marginal setulae. Male sternite 4 about 1.5 times as long as wide (Fig. 40). Male sternite 5 with moderately wide lateral lobes (Fig. 121). Cercal plate shorter than surstyli (Figs 122, 123). Aedeagus as in Fig. 137.

DISTRIBUTION. Russia: throughout midland. Europe, Mongolia [Gorodkov, 1974: 391, as S. grisea]).

Scathophaga varipes (Holmgren, 1883)

Figs 41, 124-126.

varipes Holmgren, 1883: 175 (Scatomyza). Type-locality: "Novaja Semlia" (Russia: Novaya Zemlya).

septentrionalis Becker, 1897: 397 (Scatophaga). Type-locality: Mt. Chernyshev, Novaya Zemlya (Russia).

REMARK. The species was recorded in Russia from Novaya Zemlya, Dolgii I., Kolguev I. [Holmgren, 1883: 175; Becker, 1897: 397; Gorodkov, 1970: 451, 1986: 34; Ovchinnikov, Makarova, 2016: 217], Taimyr Peninsula [Ozerov, Barkalov, 2014: 564], Yakutia [Bagachanova et al., 2016: 783], Chukotka, including Vrangel I. [Gorodkov, 1986: 34; Ozerov, Krivosheina, 2014: 219], arctic zone of European part, Siberia and Yakutia [Engelmark, 1999: 158, 159].

MATERIAL EXAMINED. Arkhangelsk Oblast: Novaya Zemlya, Matochkin Shar $\left(73.349^{\circ} \mathrm{N} 55.354^{\circ} \mathrm{E}\right), 1-5$.VIII.1889, K

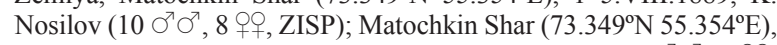
Pan'kovskaya lowland, 11-12.VIII.1889, K. Nosilov (14 $\sigma^{7} \sigma^{7}, 9$ 우, ZISP); Matochkin Shar $\left(73.349^{\circ} \mathrm{N} 55.354^{\circ} \mathrm{E}\right)$, Nochuev stream, 11.VII.-1.VIII.1925, Vakulenko (11 $\sigma^{\top} \sigma^{\top}, 8$ oO, ZISP); Chukotka: Koluychin I. $\left(67.464^{\circ} \mathrm{N} 174.617^{\circ} \mathrm{W}\right), 25-27 . V I I .1938$, Druzhinin (1 $\sigma^{7}$, ZISP); Shmidt $\left(68.870^{\circ} \mathrm{N} 179.374^{\circ} \mathrm{W}\right), 18-19 . \mathrm{VII} .1963$,

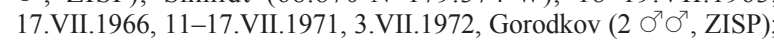
Krasnoyarsk Krai: Dikson $\left(73.508^{\circ} \mathrm{N} 80.529^{\circ} \mathrm{E}\right)$, 8.VIII.1967,

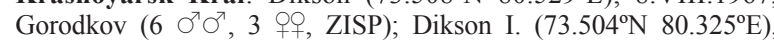
29.VII.1948, Korotkevich (4 $\Im^{7} \sigma^{7}, 1$ \%, ZISP); Lake Engel'gard $\left(75.101^{\circ} \mathrm{N} 100.234^{\circ} \mathrm{E}\right), 2 . \mathrm{VIII} .1967$, Gorodkov (6 $\sigma^{7} \sigma^{7}, 3$ 우, ZISP); Khatanga Distr. $\left(75.951^{\circ} \mathrm{N} 103.652^{\circ} \mathrm{E}\right), 10$. VII.-10.IX.2010, A. Shmanyak (2 $\sigma^{\top} \sigma^{\top}, 1$, ZMUM); Yakutia: Anabarsky Bay (ca. $\left.73.502^{\circ} \mathrm{N} 113.551^{\circ} \mathrm{E}\right), 28 . V I .1959$ (1 $\sigma^{7}$, ZMUM); Novosibirskie Islands, Blizhniy I. [= Bol'shoy Lyakhovsky I. (73.585 $\left.{ }^{\circ} \mathrm{N} 142.163^{\circ} \mathrm{E}\right)$ ],

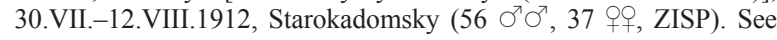
also Ozerov \& Krivosheina [2014: 219] and Ozerov \& Barkalov [2014: 564]. $7.0 \mathrm{~mm}$.

DESCRIPTION. Male. Female. Body-length 4.3-

Head. Frontal vitta yellowish-red, with delicate whitish reflection; fronto-orbital plate black, greyish dusted. Face, parafacial and gena yellow, with golden reflection. Postcranium black, densely greenish-grey dusted, but median occipital sclerite usually with blackish reflection. 3 orbital and 3-5 frontal setae present. Scapus and pedicel reddish, but blackish dorsally. Postpedicel black, but reddish basally or black completely, approximately 2 times as long as wide. Arista bare. Palpus yellow.

Thorax black, densely greenish-grey dusted. Acrostichals setulose in two rows, dorsocentrals $(2-3)+4$, intra-alars $1+2$, supra-alars $1+2$, postpronotals 2 . Anepimeron covered with hairs. Scutellum black, greenishgrey dusted, with a pair of strong basal scutellar and a pair of strong apical scutellar setae, also with a pair of discal setulae.

Legs delicately greyish dusted. All coxae black; all femora black, but reddish apically or reddish completely; all tibiae and tarsi yellow. Male and female fore femur, mid and hind male femora without conspicuous setae. Male fore tibia without conspicuous setae. Female fore tibia with 2-3 anterodorsal, 0-2 posterodorsal, 1-2 posterior, 1 preapical dorsal, and 1 posterior apical setae. Female mid femur with a row of anterodorsal setae, also with 1 preapical posterior and 1 preapical posterodorsal setae. Mid tibia with 2-3 posterodorsal, 2 anterodorsal, 1-2 anteroventral/ventral, 1-2 posterior setae and a ring of apicals in both sexes. Female hind femur with a row of anterodorsal setae and a row of anteroventral setae in apical half. Hind tibia with 3-4 anterodorsal, 2-4 posterodorsal, 3-4 anteroventral, 1 preapical dorsal, 1 preapical anterodorsal, 1 apical anterior, 1 apical anteroventral, and 1 apical ventral setae in both sexes.

Wing tinged with brownish; crossveins r-m and d-m strongly darkened.

Abdomen black, greenish-grey dusted, covered with hairs in male, without marginal setae or setulae in both sexes. Male sternite 4 about 1.5 times as long as wide (Fig. 41); sternite 5 with wide triangular lateral lobes, without median processes (Fig. 124). Epandrium, cercal plate and surstyli as in Figs 125, 126.

DISTRIBUTION. Russia: northern tundra from Siberia to Chukotka; Novaya Zemlya. - North America.

\section{Scathophaga yakutica Ozerov, 2017} Figs 42, 127-129.

yakutica Ozerov, 2017: 132 (Scathophaga). Type-locality: Aldan Plateau, right bank of the River Russkaya (ca. $58.56^{\circ} \mathrm{N}$, $\left.126.56^{\circ} \mathrm{E}\right), 60 \mathrm{~km} \mathrm{SE}$ of Tommot (Russia: Yakutia).

REMARK. This species was recorded in Russia from type locality only [Ozerov, 2017: 132].

MATERIAL EXAMINED. Buryatia: Barguzinsky Nature Reserve (ca. $\left.54.35^{\circ} \mathrm{N} 109.51^{\circ} \mathrm{E}\right), 16$. VII.1965, Negrobov (1 o, ZISP); Tyumen' Oblast: Neroyka (ca. $\left.64.57^{\circ} \mathrm{N} 59.67^{\circ} \mathrm{E}\right), 450 \mathrm{~m}$, 5.IX.1990, Malozemov (1 ア , ZISP).

DESCRIPTION. Male. Female. Body-length 6.5$7.6 \mathrm{~mm}$.

Head. Frontal vitta yellow or reddish-yellow, with delicate whitish reflection; fronto-orbital plate blackish, greyish dusted. Face, parafacial and gena yellow, with whitish reflection. Postcranium black in upper quarter or third and yellow in lower part. 3 orbital and 3 frontal setae present. Scapus and pedicel reddishyellow. Postpedicel black, approximately twice as long as wide. Arista bare. Palpus yellow. 
Thorax greyish dusted. Scutum black completely or postpronotal lobe yellowish; pleural sclerites yellowish in ground color, but anatergite, katatergite, and mediotergite always black. Acrostichals setulose in two rows, but prescutellar pair stronger than other acrostichals, dorsocentrals $2+3$, intra-alars $1+2$, supra-alars $(0+1)+2$, postpronotals 2. Anepimeron bare. Scutellum black, greyish dusted, with a pair of strong basal scutellar and a pair of strong apical scutellar setae.

Legs. Coxae from yellow to brown. Femora, tibiae, and tarsi yellowish, in male fore femur darkened posteriorly or posterodorsally. Fore femur with whitish hairs, longer ventrally, in female with a row of dorsal/posterodorsal setae, in male with 4-5 dorsal/posterodorsal setae in apical third. Fore tibia with 2 dorsal, 1-2 posterior, 1 preapical dorsal, and 1 posterior apical setae. Mid femur with a row of anterior setae, also with 1 preapical posterior and 1 preapical posterodorsal setae. Mid tibia with 3 posterodorsal, 2 anterodorsal setae and a ring of apicals in both sexes, additionally with 1 ventral seta in female. Hind femur with a row of anterodorsal setae, and with $0-1$ preapical anterior seta. Hind tibia with 3-4 anterodorsal, 3 posterodorsal, 1 preapical anterodorsal, 1 preapical posterodorsal and apical anterior setae in both sexes, additionally with 2 preapical anterior setae in female.

Wing tinged with brownish; crossveins r-m and dmcu darkened.

Abdomen from yellowish to dark brownish, covered with not long hairs, tergites with black marginal setulae. Male sternites 4 approximately as long as wide, with median excision (Fig. 42); sternite 5 with narrow pointed apically lateral lobes (Fig. 127). Epandrium, cercal plate and surstyli as in Figs 128, 129.

DISTRIBUTION. Russia: Siberia (Buryatia, Tyumen' Oblast, Yakutia).

\section{Key to species of the genus SCathophaGa Meigen}

1. Wing well-developed, long and slender (e.g., Figs 1-3, 8, 9)

- Wing strongly reduced (Fig. 7). Male sternites 4 and 5, cercal plate and surstyli as in Figs 21, 64-66 S. exalata Ozerov

2. Anepimeron covered with several hairs ........................ 3

- Anepimeron bare ......................................................... 15

3. Arista pubescent or plumose, the longest hairs approximately equal or more than $1 / 2$ width of postpedicel (e.g., Fig. 4). Male sternites 4 and 5, cercal plate and surstyli as in Figs 38, 115-117 ........ S. stercoraria (Linnaeus)

- Arista bare or nearly bare (e.g., Figs 1, 3), longest hairs not exceeding greatest diameter of arista .................... 4

4. Palpus black completely or in apical half ...................... 5

- Palpus yellow completely, if palpus dark brown or with black apex, than body and legs covered with dense and crinkly hairs (S. dasythrix)

5. Tibiae black. Palpus black completely. Fronto-orbital plate besides orbital and frontal setae additionally with numerous setulae along margin of eye (Fig. 5). Male sternites 4 and 5 , cercal plate, surstyli and aedeagus as in Figs 33, 100-102, 135
- Tibiae yellow. Palpus often yellow in basal half. Frontoorbital plate with orbital and frontal setae only, without additional setulae (e.g., Fig. 4). Male sternites 4 and 5, cercal plate, surstyli and aedeagus as in Figs 31, 94-96, 134

S. mollis (Becker)

6. Crossveins r-m and dm-cu strongly darkened (e.g., Fig. 9)

Crosseins r-m and dm-custrongly darkened (e.g., Fig. 9 )

- Crossvein dm-cu, usually also crossvein $\mathrm{r}-\mathrm{m}$ not darkened (e.g., Fig. 8) .....

7. Postcranium black, densely greenish-grey dusted, but median occipital sclerite usually with blackish reflection. Gena black in posterior half. Male sternites 4 and 5, cercal plate and surstyli as in Figs 41, 124-126....

S. varipes (Holmgren)

- Postcranium densely greyish dusted, without blackish reflection below ocellar triangle. Gena yellow. Male sternites 4 and 5, cercal plate and surstyli as in Figs 35, 106-108 ............................ S. obscurinervis (Becker)

8. Scutum with hair-like acrostichals, not differentiated from the other hairs on scutum and not forming rows ........ 9

- Scutum with setulose or well-developed acrostichals, which form two good visiable rows Scutum with intra-alar setae absent. Male sternites 4 and 5, cercal plate, surstyli and aedeagus as in Figs 34, 103105, 136 ........................................ S. obscura (Fallén)

- Scutum with intra-alar setae present. Male sternites 4 and 5, cercal plate, surstyli and aedeagus as in Figs 40, 121123,137 S. tinctinervis (Becker)

10. Tibiae black or dark brown ....................................... 11

- Tibiae at least of hind legs yellow ............................. 13

11. Scutellum with 3-4 pairs of strong setae. Body and legs covered with dense and crinkly hairs. Male sternites 4 and 5, cercal plate and surstyli as in Figs 19, 58-60 ..... S. dasythrix (Becker)

- Scutellum with 2 pairs of strong setae. Body and legs covered with short or moderately long, as a rule straight hairs

12. Male tergites 6-8 and epandrium covered with black setae (Fig.139). Male sternites 4 and 5, cercal plate and surstyli as in Figs 27, 82-84 ........... S. karelica Ozerov

- Male tergites 6-8 and epandrium without such setae (Fig.138). Male sternites 4 and 5, cercal plate and surstyli as in Figs 29, 88-90 ................... S. litorea (Fallén)

13. Male hind tibia with 3-4 pairs of setae. Female: distance beteween rows of dorsocentral and acrostichal setae approximately equal to the distance between rows of acrostical setae

- Male hind tibia often without setae, or with 1-2 pairs of setae hidden among dense hairs. Female: rows of acrostichals closer to each other than to rows of dorsocental setae. Male sternites 4 and 5, cercal plate and surstyli as in Figs 16, 49-51 ............................. S. calida (Haliday)

14. Femora of all legs black. Frontal vitta often blackish in upper part. Scapus and pedicel black. Male sternites 4 and 5, cercal plate and surstyli as in Figs 29, 88-90 ..... S. litorea Fallén

- Femora of all legs often mostly yellow. Frontal vitta yellow completely. Scapus and pedicel as a rule yellowish. Male sternites 4 and 5, cercal plate and surstyli as in Figs 26, 79-81 ....................... S. intermedia (Walker)

15. Arista pubescent or plumose, the longest hairs approximately equal to $1 / 2-3 / 4$ width of postpedicel (e.g., Fig. 4)

- Arista bare or nearly bare (e.g., Fig. 1, 3), longest hairs not exceeding greatest diameter of arista .................. 22 

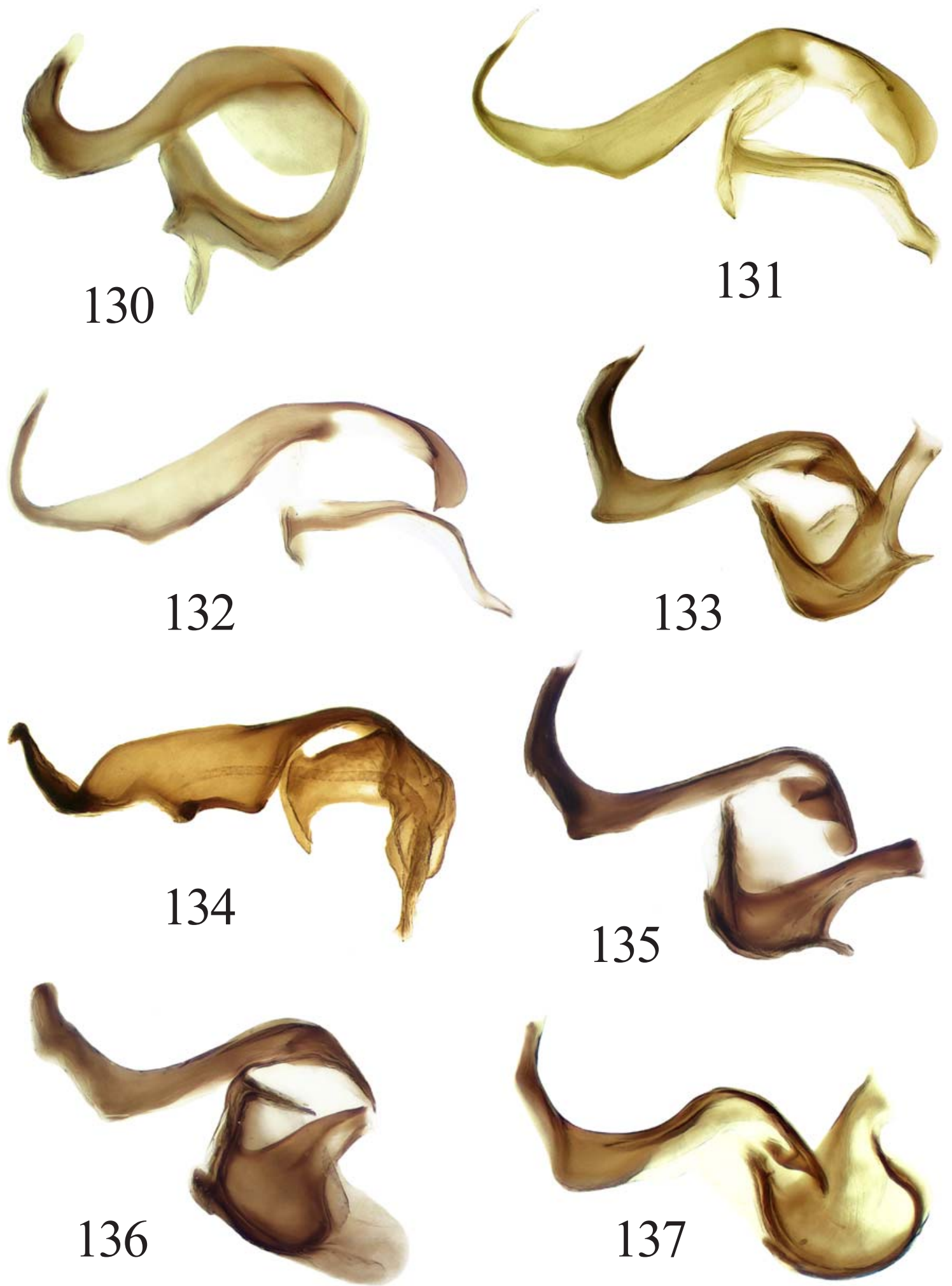

Figs 130-137. Aedeagus of Scathophaga spp., lateral view: 130 - S. buryatica Ozerov et Krivosheina; 131 - S. decipiens (Haliday); 132 - S. incompleta Ozerov et Krivosheina; 133 - S. lapponica (Ringdahl); 134 - S. mollis (Becker); 135 - S. nigripalpis (Becker); 136 - S. obscura (Fallén); 137 - S. tinctinervis (Becker). 130 - after Ozerov \& Krivosheina, 2019, fig. 2; 131, 132 — after Ozerov \& Krivosheina, 2020, figs 5, 11.

Pис. 130-137. Эдеагус Scathophaga spp., сбоку: 130 - S. buryatica Ozerov et Krivosheina; 131 - S. decipiens (Haliday); $132-$ S. incompleta Ozerov et Krivosheina; $133-$ S. lapponica (Ringdahl); $134-$ S. mollis (Becker); $135-$ S. nigripalpis (Becker); $136-S$. obscura (Fallén); 137 - S. tinctinervis (Becker). 130 - по Ozerov \& Krivosheina, 2019, fig. 2; 131, 132 — по Ozerov \& Krivosheina, 2020, figs 5, 11 . 

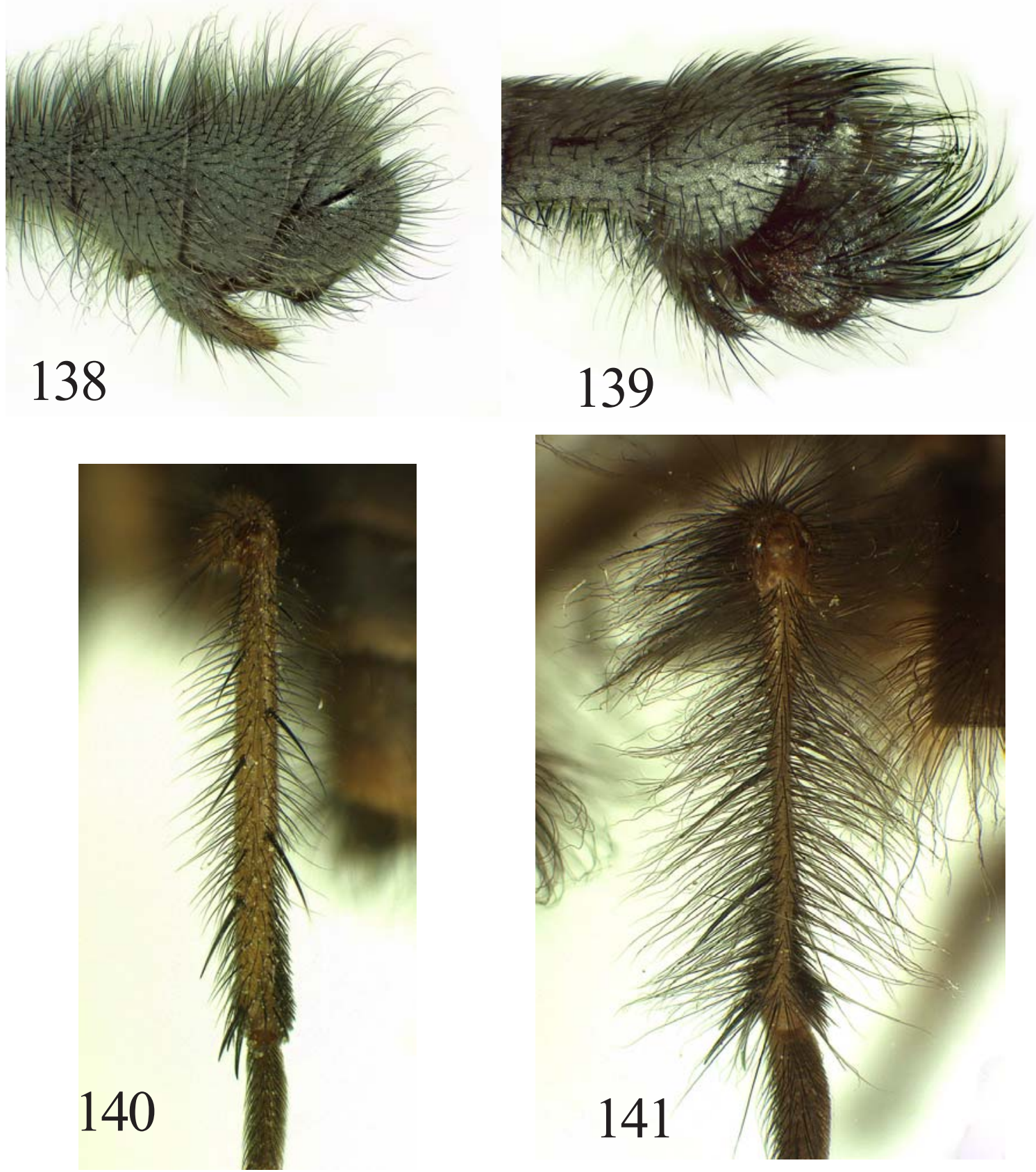

Figs 138-141. Scathophaga litorea (Fallén) (138), S. karelica Ozerov (139), S. apicalis (Curtis) (140) and S. multisetosa (Holmgren) (141): 138, 139 - end of male abdomen; 140, 141 - hind tibia, dorsal view. 138, 139 - after Ozerov, 2013, figs 11, 12.

Рис. 138-141. Scathophaga litorea (Fallén) (138), S. karelica Ozerov (139), S. apicalis (Curtis) (140) и S. multisetosa (Holmgren) (141): 138, 139 - конец брюшка самца; 140, 141 - голень задней ноги, сверху. 138, 139 - по Ozerov, 2013, figs 11, 12.

16. Postpedicel black. Legs black 17

- Postpedicel yellow, if darkened, then legs partly or completely yellow ....

17. Frontal vitta black. Scutum with well-developed acrostichals, which form two clearly visible rows. Postpedicel slightly longer than pedicel. Male sternites 4 and 5, cercal plate and surstyli as in Figs 18, 55-57

$$
\text { S. crinita (Coquillett) }
$$

- Frontal vitta reddish. Scutum with hair-like acrostichals, not differentiated from the other hairs on scutum and not forming rows. Postpedicel twice as long as pedicel. Male sternites 4 and 5, cercal plate and surstyli as in Figs 17, $52-54$. S. cordylurina (Holmgren)

18. Hind femur with $0-2$ preapical anterodorsal setae in male and with a row of anterodorsal setae in female. Female abdominal tergite 7 shiny, different from dusted tergites 5 and 6 .....

- Hind femur with a row of anterodorsal setae in both sexes. Female abdominal tergite 7 dusted as well as tergites 5 and 6 
19. Katepisternum posteriorly besides yellow hairs and setulae, as a rule also with several black setulae ............. 20

- Katepisternum posteriorly with yellow hairs and setulae, but as a rule without black setulae. Male sternites 4 and 5, cercal plate and surstyli as in Figs 37, 112-114 ........ S. spurca (Meigen)

20. Crossveins $r-m$ and $d m-c u$ strongly darkened (Fig. 9). Male sternites 4 and 5 , cercal plate and surstyli as in Figs 23, 70-72 S. incola (Becker)

- Crossvein $d m-c u$, usually also crossvein $r-m$ not darkened (e.g., Fig. 8). Male sternites 4 and 5, cercal plate and surstyli as in Figs 39, 118-120

S. taeniopa (Rondani)

21. Katepisternum posteriorly besides yellow hairs and setulae, as a rule also with several black setulae. Male sternites 4 and 5, cercal plate and surstyli as in Figs 30, 9193 .............................................. S. lutaria (Fabricius)

- Katepisternum posteriorly with yellow hairs and setulae, but as a rule without black setulae. Male sternites 4 and 5, cercal plate and surstyli as in Figs 25, 76-78.

S. inquinata (Meigen)

22. Mid and hind femora yellow completely. As a rule crossvein $d m-c u$, usually also crossvein $r-m$ darkened (e.g., Fig. 9)

- Mid and hind femora black at middle or completely. Crossveins $r-m$ and $d m-c u$ not darkened (e.g., Fig. 8) ....... 27

23. Postpedicel yellow, sometimes partly darkened (Fig. 3). Male sternites 4 and 5, cercal plate and surstyli as in Figs 22, 67-69. S. furcata (Say)

- Postpedicel black

24. Coxae of fore legs black, if yellow than postcranium black completely (S. apicalis (Curtis)) ………......... 25

- Coxae of fore legs yellow ....................................... 26

25 . Hind tibia with $2-3$ posterodorsal setae, which are as strong as anterodorsal ones (Fig. 140). Male sternites 4 and 5, cercal plate and surstyli as in Figs 14, 43-45 ..... S. apicalis (Curtis)

- Hind tibia without posterodorsal setae (males), or with posterodorsal setae which are noticeably thinner than the anterodosal setae and almost indistinguishable from the hairs (females) (Fig. 141). Male sternites 4 and 5 , cercal plate and surstyli as in Figs 32, 97-99

S. multisetosa (Holmgren)

26. Postcranium black in upper quarter or third and yellow in lower part (look at the side). Male sternites 4 and 5, cercal plate and surstyli as in Figs 42, 127-129

S. yakutica Ozerov

- Postcranium black in upper half and yellow in lower half (look at the side). Male sternites 4 and 5, cercal plate and surstyli as in Figs 36, 109-111 S. pictipennis (Oldenberg)

27. Cercal plate longer than surstyli (e.g., Figs 62, 63, 74, 75)

— Cercal plate equal to surstyli $(e . g .$, Figs $47,48,86,87) \ldots$

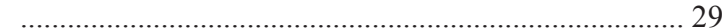

28. Scutum with slender posterior postsutural intra-alar seta or without it. Male sternites 4 and 5, cercal plate, surstyli and aedeagus as in Figs 24, 73-75, 132

S. incompleta Ozerov et Krivosheina

Scutum with two strong postsutural intra-alar setae. Male sternites 4 and 5, cercal plate, surstyli and aedeagus as in Figs 20, 61-63, 131 .................. S. decipiens (Haliday)

29. Male sternites 4 and 5 , cercal plate, surstyli and aedeagus as in Figs 28, 85-87, 133.

S. lapponica (Ringdahl)
- Male sternites 4 and 5, cercal plate, surstyli and aedeagus as in Figs 15, 46-48, 130

\section{S. buryatica Ozerov et Krivosheina}

Acknowledgements. We are very grateful to Dr. Olga Ovchinnikova and Mrs. Galina Suleymanova for the help during our work in ZISP with the material of Scathophaga. We are grateful to Dmitry I. Gavryushin (Moscow) for the use of images of living Scathophaga furcata, S. obscura and $S$. stercoraria (Figs 1-3). We are very grateful to Mr. Simon Hinkley (Melbourne Museum [formerly: National Museum of Victoria], Melbourne, Australia) for the photo of type of Scathophaga apicalis.

The investigation was fulfilled within the state project of the Institute of the Ecology and Evolution RAS (M.G. Krivosheina) and the State project No 121032300105-0 (A.L. Ozerov).

\section{References}

Albuquerque D. de O. 1984. Family Scatophagidae (Scatomyzidae, Scopeumatidae, Cordiluridae) // Papavero N. (ed.). A Catalogue of the Diptera of the Americas South of the United States. 96B. Departamento de Zoologia, Secretaria da Agricultura, São Paulo. P.1-4.

Bagachanova A.K., Ovchinnikov A.N., Ozerov A.L. 2016. On the Fauna of Scathophagidae (Diptera) of Yakutia // Entomological Review. Vol.96. No.6. P.775-785.

Becker T. 1894. Dipterologische Studien. I. Scatomyzidae // Berliner Entomologische Zeitschrift. Bd.39. H.1. S.77-196.

Becker T. 1897. Beitrag zur Dipteren-Fauna von Nowaja-Semlja // Annuaire du Musée Zoologique de l'Académie Impériale des Sciences de St. Pétersbourg. T.2. P.396-404.

Becker T. 1900. Beiträge zur Dipteren-Fauna Sibiriens. NordwestSibirische Dipteren gesammelt vom Prof. John Sahlberg aus Helsingfors im Jahre 1876 und vom Dr. E. Bergroth aus Tammerfors im Jahre 1877 // Acta Societatis Scientiarum Fennicae. T.26. No.9. S.1-66.

Becker T. 1907. Ein Beitrag zur Kenntnis der Dipterenfauna Nordsibiriens // Mémoires de l'Académie Impériale des Sciences de St. Pétersbourg, Classe Physico-mathématique. T.18. P.1-6.

Becker T. 1915. Cyclorrhapha schizophora. Holometopa // Becker Th., Dziedzicki H., Schnabl J., Villeneuve J. Résultats scientifiques de l'Expédition des frères Kuznecov (Kouznetzov) à l'Oural Arctique en 1909, sous la direction de H. Baklund. Livr.7. Diptera // Mémoires de l'Académie Impériale des Sciences, Classe Physico-Mathématique. Série 8. T.28. No.7. P.6367.

Bernasconi M.V., Pawlowski J., Valsangiacomo C., Piffaretti J.-C., Ward P.I. 2000. Phylogeny of the Scathophagidae (Diptera, Calyptratae) based on mitochondrial DNA sequences // Molecular Phylogenetics and Evolution. Vol.16. No.2. P.308-315.

Bernasconi M.V., Pawlowski J., Valsangiacomo C., Piffaretti J.-C., Ward P.I. 2001. Phylogeny of the genus Scathophaga (Diptera: Scathophagidae) inferred from mitochondrial DNA sequences. // Canadian Journal of Zoology. Vol.79. P.517-524.

Coquillett D.W. 1901. New Diptera in the U. S. National Museum // Proceedings of the United States National Museum. Vol.23. No.1225. P.593-618.

Cumming J.M., Wood D.M. 2009. Adult morphology and terminology // Brown B.V., Borkent A., Cumming J.M., Wood D.M., Woodley N.E., Zumbado M. (eds.). Manual of Central American Diptera. Vol.1. National Research Council Press, Ottawa. P.9-50.

Curtis J. 1832. British entomology; being illustrations and descriptions of the genera of insects found in Great Britain and Ireland: containing coloured figures from nature of the most rare and beautiful species, and in many instances of the plants upon which they are found. London. Vol.9. Plates 386-433 (with text). 
Engelmark R. 1999. Dungflies (Diptera: Scathophagidae) collected by the Swedish-Russian tundra ecology expedition '94, with the description of two new species; Nanna indotatum and Cochliarium sibiricum // Entomologisk Tidskrift. Arg.120. Häft 4. P.157-167.

Fabricius J.C. 1794. Entomologia systematica emendata et aucta. Secundum classes, ordines, genera, species, adjectis synonimis, locis, observationibus, descriptionibus. Tom. IV. Hafniae: C.G. Proft, Fil. et Soc. 434 pp.

Fallén C.F. 1819. Scatomyzides. Lundae. 10 pp.

Ferrar P. 1987. A guide to the breeding habits and immature stages of Diptera Cyclorrhapha // Entomonograph. Vol.8. Part.1-2. 907 pp.

Gorodkov K.B. 1970. [Family Scathophagidae (Cordyluridae, Scatomyzidae, Scopeumatidae)] // Bei-Bienko G.Ya. (ed.). Opredelitel' nasekomykh Evropeiskoi Chasti SSSR. Vol.5. Diptera, Siphonaptera. Part 2. P.440-458 [in Russian].

Gorodkov K.B. 1974. [Scatophagidae (Diptera) of Mongolian People's Republic] // Nasekomye Mongolii. T.2. P.380-395 [in Russian].

Gorodkov K.B. 1978. [Diptera, Scathophagidae. Scathophaga furcata (Say, 1823)]. Map 20. P.24 // Arealy nasekomykh Evropeiskoy chasti SSSR. Maps 1-20. Leningrad: Nauka Publ. [in Russian].

Gorodkov K.B. 1980. [Map 68. Scathophaga suilla (Fabricius, 1794). Diptera, Scathophagidae] // Arealy nasekomykh Evropeiskoy chasti SSSR. Atlas. Maps 21-72. Leningrad: Nauka Publ. P.51 [in Russian].

Gorodkov K.B. 1981. [Map 121. Scathophaga stercoraria (Linnaeus, 1758). Diptera, Scathophagidae] // Arealy nasekomykh Evropeiskoy chasti SSSR. Atlas. Maps 73-125. Leningrad: Nauka Publ. P.52 [in Russian].

Gorodkov K.B. 1986. Family Scathophagidae // Soós Á., Papp L. (eds.). Catalogue of Palaearctic Diptera. Vol.11. ScathophagidaeHypodermatidae. Budapest: Akadémiai Kiadó. P.11-41.

Hendel F. 1930. Entomologische Ergebnisse der schwedischen Kamtchatka-Expedition 1920-1922. 28. Diptera Brachycera 2. Fam. Cordyluridae und Dryomyzidae // Arkiv för Zoologi. Bd.21A. Nr.18. S.1-12.

Holmgren A.E. 1869. Bidrag till kännedomen om Beeren Eilands och Spetsbergens insekt-fauna // Kongliga Svenska Vetenskaps-Akademiens Handlingar. Vol.8. Part 5. S.3-55.

Holmgren A.E. 1880. Novas species insectorum cura et labore A. E. Nordenskiöldii e Novaia Semlia coactorum. Holmiae [= Stockholm]: Beckmanniana. 24 pp.

Holmgren A.E. 1883. Diptera // Holmgren A.E., Aurivillius C. Insecta a viris doctissimis Nordenskiold illum ducem sequentibus in insulis Waigatsch et Novaja Semlia anno 1878 collecta. Hymenoptera et Diptera // Entomologisk Tidskrift. Haft 4. S. 162-190

Humala A.E., Polevoi A.V. 2009. On the insect fauna of south-east Karelia // Trudy Karelskogo nauchnogo tsentra RAN. Vol.4. P.53-75.

Illiger J.C.W. 1807. Favna Etrvsca sistens Insecta quae in provinciis Florentina et Pisana praesertim collegit Petrvs Rossivs. Tomvs Secvndvs. Cvm IX Tab. Itervm edita et annotatis perpetvis avcta a D. Carolo Illiger. Helmstadii, C.G. Fleckeisen, vi + 511 pp. + tables.

Jong H., de. 2000. Family Scathophagidae // Papp L., Darvas B. (eds.). Contributions to a Manual of Palaearctic Diptera. Appendix. Budapest: Science Herald. P.431-445.

Linnaeus C. 1758. Systema naturae per regna tria naturae. Ed.10. Holmiae. Vol.L. 824 pp.

Lundbeck W. 1901. Diptera groenlandica [II]. // Videnskabelige Meddelelser fra Dansk Naturhistorisk Forening. Vol.52(1900). P. 281-316.

McAlpine J.F. 1981. Morphology and terminology-adults // McAlpine J.F., Peterson B.V., Shewell G.E, Teskey H.J., Vockeroth J.R., Wood D.M. (Coordinators). Manual of Nearctic Diptera. Vol.2. Research Branch. Agriculture Canada. Monograph 27, Ottawa. P.9-63.

MacGowan I., Vikhrev N.E., Krivosheina M.G., Ruchin A.B. Esin M.N. 2021. New records of Diptera from the Republic of Mordovia, Russia // Far Eastern Entomologist. No.423. P.9-20.

Meigen J.W. 1800. Nouvelle classification des mouches à deux ailes (Diptera L.) d'après un plan tout nouveau. Paris. 44 pp.

Meigen J.W. 1803. Versuch einer neuen Gattung-seintheilung der europäischen zweiflügeligen Insekten // Magazin für Insektenkunde. Theil.2. S.259-281.

Meigen J.W. 1826. Systematische Beschreibung der bekannten europäischen zweiflügeligen Insecten. Fünfter Theil. [5]. Hamm. $412 \mathrm{~S}$.

Meijere J.C.H. de. 1907. Eerste Supplement op de Nieuwe Naamlijst van Nederlandsche Diptera // Tijdschrift voor Entomologie. Vol.50. S.151-195.

Michelsen V., O’Hara J.E. 2014. A review of genus-group names in Diptera (Insecta) that J.C. Fabricius "borrowed" from other dipterists and proposed as new in his systematic works from 1775 to 1805 // Zootaxa. Vol.3873. No.1. P.073-081.

Oldenberg L. 1923. Neue Acalyptraten (Diptera) meiner Ausbeute // Deutsche Entomologische Zeitschrift. Bd.3. S.307-319.

Ovchinnikov A.N. 2004. Data on the fauna of Scathophagidae (Diptera) of the Volga Region // Russian Entomological Journal. Vol.12. No.4(2003). P.421-423.

Ovchinnikov A.N. 2009. The Ovipositor Morphology in the Members of the Family Scathophagidae (Diptera) with Reference to Their Biology // Entomological Review. Vol.88. No.2. P.314-328.

Ovchinnikov A.N., Makarova O.L. 2016. The dung flies (Scathophagidae, Diptera) of the Dolgii Island, Barents Sea // Russian Entomological Journal. Vol.25. No.2. P.215-218.

Ozerov A.L. 1996. Scathophaga exalata sp.n. (Diptera, Scathophagidae) with reduced wings from Kuril Islands // Far Eastern Entomologist. No.35. P.1-4.

Ozerov A.L. 2009. Fam. Scathophagidae // Storozhenko S.Yu. et al. (eds.). Nasekomye Lazovskogo zapovednika. Vladivostok: Dalnauka. P.379 [in Russian].

Ozerov A.L. 2010a. Review of Afrotropical Scathophagidae (Diptera) // Russian Entomological Journal. Vol.18. No.4(2009). P.299-308.

Ozerov A.L. 2010b. Five new species of Scathophagidae (Diptera) from Russia // Russian Entomological Journal. Vol.19. No.2. P.157-166.

Ozerov A.L. 2012. Scathophaga helenae (Thomson, 1869), a new synonym of Scathophaga soror (Wiedemann, 1818) (Diptera: Scathophagidae) // Far Eastern Entomologist. No.247. P.14-16.

Ozerov A.L. 2013. Description of five new species and notes on taxonomy of Scathophagidae (Diptera) // Russian Entomological Journal. Vol.22. No.1. P.81-89.

Ozerov A.L. 2017. A new species of the genus Scathophaga Meigen, 1803 (Diptera: Scathophagidae) from Yakutia (Russia) // Entomological Review. Vol.97. No.1. P.132-135.

Ozerov A.L., Barkalov A.V. 2014. On the fauna of Scathophagidae (Diptera) of Taimyr Peninsular (Russia: Krasnoyarskiy Kray) // Euroasian Entomological Jornal. Vol.13. No.6. P.560-565.

Ozerov A.L., Freidberg A. 2011. The Scathophagidae (Diptera) of Israel // Israel Journal of Entomology. Vol.40 (2010). P.169185.

Ozerov A.L., Krivosheina M.G. 2011. To the knowledge of dung flies (Diptera, Scathophagidae) of Vietnam, with the description of a new species // Far Eastern Entomologist. No.228. P.1-10.

Ozerov A.L., Krivosheina M.G. 2014. To the fauna of dung flies (Diptera: Scathophagidae ) of Russian Far East // Russian Entomological Journal. Vol.23. No.3. P.203-222.

Ozerov A.L., Krivosheina M.G. 2019. Two new species of the genus Scathophaga Meigen, 1803 (Diptera: Scathophagidae) from Asia // Russian Entomological Journal. Vol.28. No.1. P.102-106.

Ozerov A.L., Krivosheina M.G. 2020. To taxonomy of the genus Scathophaga Meigen, 1803 (Diptera: Scathophagidae) with description of a new species // Russian Entomological Journal. Vol.29. No.2. P.227-231.

Perris E. 1839. Notice sur quelques Diptères nouveaux. // Annales de la Société Entomologique de France. Vol.8. P.47-57. 
Polevoi A. 1997. Diptera collected with Malaise traps in the Kostomuksha Nature Reserve // Lindholm T., Heikkilä R., Heikkilä M. (eds.). Ecosystems, fauna and flora of the Finnish-Russian Nature Reserve Friendship, Helsinki. P.303-309.

Ringdahl O. 1920. Neue skandinavische Dipteren // Entomologisk Tidskrift. Vol.41. S.24-40.

Ringdahl O. 1936. Anteckningar till svenska arter av familjen Scopeumatidae (Diptera) // Entomologisk Tidskrift. Arg.57. Haft. 2-3. S.158-179.

Ringdahl O. 1937. Eine Namensänderung // Entomologisk Tidskrift. Arg.58. S.38.

Robineau-Desvoidy J.B. 1830. Essai sur les Myodaires // Mémoires présentés par divers Savans à l'Académie Royale des Sciences de l'Institut de France. Paris. T.2. P.1-813.

Rondani C. 1866. Scatophaginae Italicae collectae distinctae et in ordinem dispositae. Dipterolodiae Italicae Prodromus. Pars. VII. Fasc.I. Bernardoni, Milano. 52 pp. [Also published under the same title in Atti della Società Italiana di Scienze Naturali. 1867. Vol.10. P.85-135].

Ross J. 1835. Appendix to the Narrative of a Second Voyage in search of a North-West Passage, and of a residence in the Arctic regions during the years 1829, 1830, 1831, 1832, 1833. Including the reports of Commander, now Captain, James Clark Ross, R.N., F.R.S., F.L.S. \& c. and the discovery of the northern magnetic pole. xii $+120+$ cxliv + cii pp., 20 plates. Webster, London. [The report on "Natural History" is on pp. i$\mathrm{c}$ in the first post-report section with Roman numerals.]

Sack P. 1937. Die Fliegen der Palaearktischen Region. 62a. Cordyluridae. (Scatomyzidae) // Lindner E. (Hrsg.). Die Fliegen der palaearktischen Region, Stuttgart. Vol.7. $103 \mathrm{~S}$.

Say T. 1823. Description of dipterous insects of the United States // Journal of the Academy of Natural Sciences of Philadelphia. Vol.3. P.9-54, 73-104.

Séguy E. 1952. Diptera fam. Scatophagidae // Wytsman P. (ed.). Genera Insectorum. Desmett-Verteneuil Imprimeur-Editeur, Brussels. P.1-107.

Šifner F. 2008. A catalogue of the Scathophagidae (Diptera) of the Palaearctic region, with notes on their taxonomy and faunistics // Acta Entomologica Musei Nationalis Pragae. Vol.48. No.1. P.111-196.

Šifner F. 2013. Coniosternum kovari sp. nov. from Tajikistan, with the key to Palaearctic species of Coniosternum (Diptera: Scathophagidae) // Klapalekiana. Vol.49. P.205-218.

Šifner F. 2017. Coniosternum masneri sp. nov., a new species of the family Scathophagidae (Diptera) from Canada // Journal of the National Museum (Prague), Natural History Series. Vol.186. P.113-116.

Šifner F. 2018. Two new species of the genus Scathophaga (Diptera: Scathophagidae) from Svalbard, with a key to selected northern species and comments on their taxonomy // Journal of the National Museum (Prague), Natural History Series. Vol.187. P. $27-46$.
Šifner F. 2020. Scathophaga karmazini nov.sp., a new species from Austria, with a key to Scathophaga species from Central Europe (Diptera, Scathophagidae) // Linzer Biologische Beiträge. Vol.52. No.1. P.731-757.

Strobl G. 1894. Die Dipteren von Steiermark. II. Theil. // Mitteilungen des Naturwissenschaftlichen Vereines für Steiermark. Bd.30. S.1-152.

Stuckenberg B.R. 1999. Antennal evolution in the Brachycera (Diptera), with a reassessment of terminology relating to the flagellum // Studia Dipterologica. Vol.6. S.33-48.

Sun X. 1996. Scathophagidae // Xue Wanqi (Hsue Wan-chi), Chao Chienming (eds.). Flies of China. Vol.1. Shenyang: Liaoning Science and Technology Press. P.622-633.

Sychevskaya V.I. 1972. [On the synanthropic flies of Yakutia] // Ammosov Yu.N. (ed.). Fauna i ekologiya nasekomykh Yakutii. Yakutsk. P.144-157 [in Russian].

Szilády Z. 1926. Dipterenstudien. II. Bemerkungen über einige Scatophagiden des Ungarischen National Museum // Annales historico-naturales Musei nationalis hungarici. T.24. P.593-597.

Verves Yu.G., Zrazhevskii S.F., Tsibulskii A.I. 1990. [Some groups of Diptera of the Ust-Lenskii Nature Reserve and adjacent territories] // Nukhimovskaya Yu.D., Bibikova L.A. (eds.). Antropogennye vozdeistviya na prirodu zapovednikov. Moscow. P.136-142 [in Russian].

Veselkin G.A. 1985. [Peculiarities of the fauna and ecology of zoophilous flies of Yakutia] // Solomonov N.G. (ed.). Materialy po faune i ekologii nasekomykh Yakutii. Yakutsk. P.73-79 [in Russian].

Villeneuve J. 1917. Description de deux Muscides nouveaux (Dipt.) // Bulletin de la Société Entomologique de France. No.17. P.306-309.

Vockeroth J.R. 1965. Subfamily Scatophaginae // Stone A. et al. (eds.). A catalog of the Diptera of America north of Mexico. United States Department of Agriculture, Agriculture Handbook. No.276. P.826-842.

Vockeroth J.R. 1977. Family Scathophagidae // Delfinado M.D., Hardy D.E. (eds.). A Catalog of the Diptera of the Oriental Region. Vol.3. Suborder Cyclorrhapha (excluding Division Aschiza). Honolulu: the University Press of Hawaii. P.436-438.

Vockeroth J.R. 1987. 103. Scathophagidae // McAlpine J.F. et al. (eds.). Manual of Nearctic Diptera. 2. Agriculture Canada, Research Branch, Ottawa, Monograph P.1085-1097.

Vockeroth J.R. 2010. 105. Scathophagidae (Dung flies) // Brown B.V. et al. (eds.). Manual of Central American Diptera. Vol.2. Ottawa: NRC Research Press. P.1267-1269.

Walker F. 1849. List of the specimens of dipterous insects in the collection of the British Museum. London. Vol.4. P.689-1172.

Zetterstedt J.W. [1838]. Sectio tertia. Diptera // Insecta Lapponica.' 1840 ', Leopold Voss, Lipsiae [= Leipzig]. P.477-868.

Zetterstedt J.W. 1846. Diptera Scandinaviae disposita et descripta. Tomus quintus. Ex officina lundbergiana, Lundae [= Lund]. P.1739-2162. 